\title{
Assessing the health-related outcomes and correlates of active transportation in children and youth
}

Richard Larouche

Thesis submitted to the

Faculty of Graduate and Postdoctoral Studies

in partial fulfillment of the requirements

for the Doctorate in Philosophy degree in Human Kinetics

School of Human Kinetics

Faculty of Health Sciences

University of Ottawa

(C) Richard Larouche, Ottawa, Canada, 2013 


\section{Table of contents}

\section{Section}

\section{Page}

List of tables

$\mathrm{V}$

List of figures

vii

Abstract

viii

Acknowledgements

ix

Chapter 1. Introduction

Active transportation

The "physical inactivity crisis"

Active transportation as a source of physical activity

Health-related outcomes of active transportation

The mode share of active transportation

Correlates of active transportation

Knowledge gaps

Objectives and research questions

Organization of the thesis

Chapter 2. Manuscript I: "Associations between active school transport and physical activity, body composition and cardiovascular fitness: a systematic review of 68 studies"

Chapter 3. Manuscript II: "Relationship between active school transport and body mass index in Grades 4-to-6 children" 
activity, fitness and cardiovascular disease risk factors: findings from the

Canadian Health Measures Survey"

Chapter 5. Manuscript IV: "Assessing the influence of the transition from

primary to secondary school on the volume of active school transport and physical activity: a prospective pilot-study"

Chapter 6. Manuscript V: "Correlates of active school travel immediately before and after the transition from primary to secondary school: a pilot-study"

Chapter 7. Manuscript VI: “Associations between neighbourhood walkability, active school transport and physical activity levels in primary and secondary school students: a pilot-study"

Chapter 8. General discussion

Relationships of active school transport with physical activity and healthrelated outcomes

Correlates of active school transport

Limitations and strengths of the studies

Direction for future research

Implications for interventions and policies

References 


\begin{tabular}{lc}
\hline Section & Page \\
\hline Appendix 3. University of Ottawa Research Ethics Board (REB) approval & 271 \\
Appendix 4. Children's Hospital of Eastern Ontario REB approval & 273 \\
Appendix 5. Ottawa-Carleton Research Advisory Committee approval & 274 \\
Appendix 6. Transportation diary & 275 \\
Appendix 7. Pedometer log sheet & 276 \\
Appendix 8. Parent/guardian travel questionnaire & 277 \\
Appendix 9. Child questionnaire on perceived barriers to active school transport & 281 \\
Appendix 10. Internal consistency of perceived barriers to active school & 282 \\
\hline
\end{tabular}




\section{List of tables}

Title

Page

Chapter 1. Table 1. Overview of the studies

Chapter 2. Table 1. Characteristics of included studies

64

Chapter 2. Table 2. Outcomes of studies examining differences in physical activity

levels between active and passive travelers

Chapter 2. Table 3. Outcomes of studies examining differences in body composition

between active and passive travelers

Chapter 2. Table 4. Outcomes of studies examining differences in cardiovascular fitness

75

between active and passive travelers

Chapter 2. Table 5. Quality assessment ratings according to the Effective Public Health

76

Practice Project tool for quantitative studies

Chapter 3. Table 1. Descriptive characteristics of the sample

Chapter 3. Table 2. Results of the ANCOVA analyses for body mass index and waist circumference

Chapter 4. Table 1. Descriptive characteristics of participants to the 2007-2009 115

Canadian Health Measures Survey stratified by gender

Chapter 4. Table 2. Associations of levels of walking with gender, age group and parental education levels

Chapter 4. Table 3. Associations of levels of cycling with gender, age group and parental education levels

Chapter 4. Table 4. Associations between walking for transportation and health-related outcomes in adolescents 

outcomes in adolescents

Chapter 5. Table 1. Descriptive characteristics of the sample at baseline and follow-up

Chapter 5. Table 2. Changes in active school transport, distance between home and school, and average daily step counts upon the transition from primary to secondary school

Chapter 5. Table 3. Differences in average daily step counts between active and inactive travelers

Chapter 6. Table 1. Descriptive characteristics of the sample

Chapter 6. Table 2. Spearman correlations between child- and parent-perceived barriers to active transportation at baseline and follow-up

Chapter 6. Table 3. Associations between child-perceived barriers and active school transport at baseline and follow-up

Chapter 6. Table 4. Associations between parental neighborhood selection factors and child's travel mode at baseline and follow-up

Chapter 6. Table 5. Associations between socio-demographic characteristics of the household and children's travel mode at baseline and follow-up

Chapter 7. Table 1. Descriptive characteristics of the sample at baseline and follow-up primary travel mode at baseline and follow-up Chapter 7. Table 3. Associations between neighbourhood walkability and step counts at baseline and follow-up

Chapter 8. Table 1. Overview of the main study findings 


\section{List of figures}

Title Page

Chapter 1. Figure 1. Conceptual framework

20

Chapter 2. Figure 1. Flow of articles through the search process

Chapter 5. Figure 1. Flow of participants in the Active Transportation Transition Study 


\begin{abstract}
Active school transport (AST; e.g. the use of non-motorized modes such as walking and cycling to travel to/from school) is an inexpensive, accessible and environmentally-friendly source of physical activity (PA). This dissertation addresses two overarching objectives: 1) to measure the relationships of AST with PA and health-related outcomes; and 2) to examine the correlates of AST immediately before and after the transition from primary to secondary school (the "school transition"). First, a systematic review revealed increasing evidence showing that AST is associated with greater daily PA levels, and that cycling to/from school is associated with higher cardiovascular fitness. Cycling for transportation (not only for school trips) was also associated with lower values for total cholesterol and total cholesterol/HDL cholesterol ratio in the nationally-representative 2007-2009 Canadian Health Measures Survey. Moreover, the present dissertation provides preliminary evidence suggesting that AST may help attenuate the decline in PA across the school transition. However, the relationship between AST and body composition indicators remains unclear. With respect to the correlates of AST, distance was the strongest barrier to AST at both time points, but several road safety concerns, and the perception of having too much stuff to carry were also associated with engagement in motorized travel. At follow-up, AST was more common in children whose parents owned less than 2 cars. In contrast, children were more likely to engage in AST if their parents reported that they chose to live in their current neighbourhood so that their children could walk or bike to school. The associations of neighbourhood walkability (as measured with the Walk Score ${ }^{\circledR}$ application) with AST and PA were generally stronger after the school transition. While AST may improve health among children and youth, an ecological approach targeting multiple levels of influence will likely be needed to alleviate current barriers to AST.
\end{abstract}




\section{Acknowledgements}

First, I would like to acknowledge the constant support from my supervisor, Dr. Mark Tremblay, during my progress in the Ph.D. program. Dr. Tremblay is not only a skillful researcher, but also a great mentor. He really deserved the prize for mentorship that he was awarded by the Children's Hospital of Eastern Ontario Research Institute in 2012.

Second, I would like to acknowledge the invaluable feedback from my committee members (Drs. Guy Faulkner and Michelle Fortier) on my thesis proposal and on the manuscripts.

I would also like to thank Dr. Meghann Lloyd for offering me the opportunity to work on the Canadian Assessment of Physical Literacy study and to analyze some of the data.

I am grateful for the advice of Dr. Nick Barrowman for statistical analyses, and Dr. Margaret Sampson for the development of the search strategy for my systematic review. I have also appreciated the important contribution of the 10 key informants that we consulted for the systematic review.

I also feel privileged to be part of the Healthy Active Living and Obesity Research Group at the Children's Hospital of Eastern Ontario Research Institute. I would like to thank all the investigators, students, research assistants and administrative assistants. 
I would also like to acknowledge the Canadian Institutes of Health Research for awarding me a Frederick Banting and Charles Best Doctoral Award, and the University of Ottawa for my Excellence scholarship as well as for research and teaching assistantships.

Last but not least, the support of my family and friends has been instrumental during the last four years, in the good and the bad times. 


\section{Chapter 1. Introduction}

\subsection{Active transportation.}

During the last decade, active transportation (e.g. using modes such as walking and cycling to travel to and from places such as work, school, parks, and shops) has emerged as an important topic in the fields of public health, urban planning and environmental sciences (British Medical Association, 2012; de Nazelle et al., 2011; Haines et al., 2010; Larouche, 2012; Litman, 2013; Sallis et al., 2006; Shephard, 2008). Public health researchers are interested in promoting active transportation as a strategy to increase physical activity and prevent non-communicable diseases at the population level (Sallis et al., 2006; Shephard, 2008). In parallel, environmental scientists have shown that motorized travel is one of the most important contributors to rising greenhouse gases emissions and climate change (Chapman, 2007; Unger et al., 2010). Therefore, active transportation is viewed as a key climate change mitigation strategy (Haines et al., 2010; Woodcock et al., 2009). For their part, urban planners are concerned with transport system issues such as mobility and traffic congestion, travel demand and neighborhood development, all of which are likely to influence individuals' travel mode decisions (Cervero \& Kockelman, 1997; Litman, 2013). Collaborations between researchers from these different fields are becoming increasingly common as the potential for simultaneously achieving public health, urban planning and environmental objectives through active transportation is recognized (Frank et al., 2010; Litman, 2013; Maibach et al., 2009). This potential for "win-win" interventions can be attractive from a policy-maker perspective in these times of economic hardship.

To date, the majority of research on active transportation among children and youth has focussed on the trip to and from school (Panter et al., 2008); hereafter referred to as active school 
transport (AST) $)^{1}$. From a public health perspective, this is likely due to the fact that given their frequency, trips to/from school may represent an important source of physical activity (PA) for children and youth. In addition, motorized travel to school accounts for an increasingly large proportion of "rush hour" traffic and is associated with sizable emissions of exhaust and greenhouse gases (Marshall et al., 2010; McDonald et al., 2011).

\subsection{The "physical inactivity crisis".}

Public health researchers often refer to the very low levels of PA in many countries around the world as a "physical inactivity crisis" (Bauman et al., 2009; Yancey \& Sallis, 2009). Although PA guidelines recommend that children and youth engage in at least 60 minutes of daily moderate-to-vigorous physical activity (MVPA) (Tremblay et al., 2011; United States Department of Health and Human Services, 2008), the proportion of children and youth who meet this guideline is very low in many countries across the world (Basterfield et al., 2011; Colley et al., 2011; Hallal et al., 2012; Troiano et al., 2008). For example, data from the Canadian Health Measures Survey indicate that only 7\% of Canadian children and youth met the Canadian PA guidelines (Colley et al., 2011). Similarly, a study of 1,001 children aged 10 to 12 years old in the Greater Toronto Area found that only $1 \%$ of participants met the PA guidelines (Stone et al., 2013). This low compliance to PA guidelines at the population level may have important health implications.

It has been shown that insufficient PA among children and youth is linked to deleterious health outcomes such as a higher prevalence of obesity (Jimenez-Pavón et al., 2010), cardiovascular disease (CVD) risk factors (Andersen et al., 2006; Ekelund et al., 2012), and lower glucose tolerance and higher fasting insulin levels (Jago et al., 2008). Insufficient PA is

\footnotetext{
${ }^{1}$ The reader is referred to Appendix 1 for a list of abbreviations.
} 
also associated with poor cardiovascular fitness, which is a strong and independent predictor of future risk of CVDs (Anderssen et al., 2007). Conversely, increasing physical activity can lead to improved academic achievement (Hillman et al., 2008; Trudeau \& Shephard, 2008). Moreover, a review of reviews indicated that PA may also contribute to improved mental health in children and youth, although the use of more rigorous study designs is warranted (Biddle \& Asare, 2011).

During the last few decades, several studies have shown large declines in indicators of physical fitness (Craig et al., 2012; Tremblay et al., 2010; Wedderkopp et al., 2004; Westerstahl et al., 2003) accompanied by increasing obesity levels (Janssen et al., 2011; Lobstein et al., 2004; Olds, 2009), and even increasing prevalence of CVD risk factors (McCrindle et al., 2010) among children and youth. Together, this body of evidence suggests that promoting PA should be viewed as an urgent public health priority.

\subsection{Active transportation as a source of physical activity.}

Twelve years ago, Tudor-Locke, Ainsworth and Popkin (2001) described the journey to/from school as "an overlooked form of children's' physical activity" because it was largely ignored in surveys of PA despite anecdotal evidence suggesting a steep decline of the mode share of AST in several countries. Hence, interventions have traditionally focussed on leisuretime physical activity to foster an increase of total PA level. However, increasing evidence suggests that this strategy may be insufficient if not complemented by other forms of physical activity (Bauman et al., 2008; Levine et al., 1999; Tremblay et al., 2007). Since most children and youth must travel to school on a daily basis, active transportation is regarded as a promising strategy to increase PA levels (Larouche \& Trudeau, 2010; Shephard, 2008). 
During the last decade, several researchers have examined the relationship between AST and PA using different methods to assess PA (i.e. accelerometry, pedometry and questionnaires). A systematic review by Faulkner and colleagues (2009) found that AST was associated with greater objectively-measured PA levels in 11 of the 13 included studies. In studies using accelerometers, the mean difference between active and passive commuters varied from 0 to 45 minutes of daily MVPA. These differences are likely due to the use of inconsistent accelerometer cut-points to define MVPA (Cain et al., 2013). Other studies have reported that active commuters were more likely to meet PA guidelines based on self-reports (Trang et al., 2009; Tudor-Locke et al., 2002) and pedometer step counts (Abbott et al., 2009; Johnson et al., 2010). Additionally, Morency and Demers (2010) estimated that if 5-14 years old children in Montréal replaced all their motorized trips of less than $1 \mathrm{~km}$ by walking, they would accumulate an average of 2,238 additional steps/day.

\subsection{Health-related outcomes of active transportation.}

Given that active transportation is one of the components of PA, one could hypothesize that AST may yield health benefits similar to those of PA in general. Hence, several studies have assessed whether AST is related to indicators of body composition such as body mass index (BMI), waist circumference, skinfold thickness and body fat percentage. However, the most recent systematic review (Lubans et al., 2011) has noted mixed findings; specifically, $48 \%$ of studies found that AST was associated with more favorable body composition. When lower quality studies were excluded, this proportion was $55 \%$.

Aside from study quality, a few hypotheses have been proposed to explain such conflicting findings. First, the energy expenditure from AST may be insufficient to have a 
substantial impact on body weight (Lee et al., 2009). Second, researchers have used inconsistent methods to classify individuals as active vs. inactive travelers (Faulkner et al., 2009). For example, in some studies, children using AST only once per week were classified as "active travelers" whereas in other studies, only individuals using AST at least $50 \%$ of the time were classified as active travelers. Third, most studies have measured only BMI as an indicator of obesity, and this indicator may not accurately reflect body composition (Bere \& Andersen, 2009). Fourth, particularly in North American countries, AST tends to be more common among children from low socio-economic status (SES) households (Evenson et al., 2003; Pabayo et al., 2008, 2011). In turn, low SES has been found to be associated with overweight and obesity (Janssen et al., 2006; Stamatakis et al., 2010). Thus, failure to adjust for potential confounders such as SES may partially account for the inconsistent evidence. Fifth, individuals may compensate for the energy expenditure resulting from AST, either by: 1) engaging in less PA; 2) engaging in more sedentary behaviour (e.g. waking activities with energy expenditures $\leq 1.5$ metabolic equivalents); and/or 3) increasing their food intake during the rest of the day (Larouche \& Saunders, 2012).

Fewer studies have examined the relationship between AST and cardiovascular fitness (Lubans et al., 2011). From a theoretical perspective, Shephard (2008) argued that because the energy expenditure of cycling is greater than that of walking, utilitarian cycling may have a greater impact on cardiovascular fitness, especially in children and youth for whom walking may not elicit a sufficient PA intensity to increase cardiovascular fitness. In Lubans and colleagues' (2011) review, four of the five included studies found that AST - especially cycling - was associated with greater cardiovascular fitness. The study that found no relationship (e.g. Madsen et al., 2009) was the only one that did not differentiate walkers and cyclists in statistical analyses. 
These findings are supportive of Shephard's rationale, emphasizing that researchers should examine the effect of walking and cycling separately provided that their sample size is sufficient to do so.

\subsection{The mode share of active transportation.}

Despite these potential health benefits, the 2013 Active Healthy Kids Canada Report Card on Physical Activity in Children and Youth (Active Healthy Kids Canada 2013) shows that many studies have found that only 25 to $35 \%$ of Canadian children and youth regularly engage in AST. The Report Card also highlights large regional disparities. Indeed, fewer children engage in AST in rural areas, in the Atlantic provinces and in Québec, whereas AST is more common in urban areas and in the Western provinces and territories.

The proportion of children and youth using AST (typically refered to as the mode share of AST) has decreased markedly in the last few decades in several countries (Buliung et al., 2009; Costa et al. 2012; Cui et al., 2011; Grize et al., 2010; Lewis et al., 2008; McDonald et al., 2011; Trang et al., 2012; van der Ploeg et al., 2008). For example, in the Greater Toronto Area, findings from the Transportation Tomorrow Survey indicated significant decreases in the mode share of AST among youth aged 11-13 years (from $53.0 \%$ to $42.5 \%$ ) and $14-15$ years (from 38.6\% to 30.7\%) between 1986 and 2006 (Buliung et al., 2009). Similarly, in Montréal, the percentage of primary school children walking to school declined from $40.5 \%$ to $34.2 \%$ between 1998 and 2003 (Lewis et al., 2008). In the United States, the mode share of AST decreased from 47.7\% to 12.7\% between 1969 and 2009 (McDonald et al., 2011).

These trends mirror the large decreases in children's independent mobility - that is the degree of freedom of children and youth to move around in public spaces without adult 
supervision - that occured concurently (Fyhri et al., 2011; Hillman et al., 1990). For example, Hillman and colleagues (1990) reported that in 1971, 80\% of British 7-8 years olds were allowed to go to school without adult supervision, but this proportion decreased to only $9 \%$ by 1990 . This decline is worrying given that independent mobility can contribute to children's cognitive development by improving their way-finding skills and help them develop a more positive relationship with their environment (Paccili et al., 2013; Rissotto \& Tonucci, 2002).

Switching from an active travel mode to an inactive one could be an important contributor to the well-documented decline in PA levels from childhood to adolescence (Dumith et al., 2011; Sallis et al., 2000). For example, a meta-analysis of 26 tracking studies found a 7\% annual decline in PA during late "pre-teen" years and adolescence (Dumith et al., 2011). A similar decline was observed in a prospective study of adolescents in Montréal (Bélanger et al., 2009a,b). From another perspective, researchers have argued that major life events which are often associated with steep declines in PA (e.g. the transition from primary to secondary school, entry into the labour market, parenthood, etc.) may represent key periods for the promotion of PA (Allender et al., 2008; Engberg et al., 2012; Larouche et al., 2012).

To date, only one study has prospectively examined changes in AST across the transition from primary to secondary school (Cooper et al., 2012). These authors reported that fewer British children walked to/from school in secondary school, but walkers traveled greater distances in secondary school. They also reported increases in MVPA in children who started to walk to school in secondary school and in those who walked at both time points. Conversely, they noted a $15.5 \%$ decrease in MVPA in children who switched from walking to car/bus riding.

\subsection{Correlates of active school transportion.}


Researchers have examined a wide range of factors as potential correlates of AST including personal characteristics (i.e. enjoyment of walking or cycling), the social environment (i.e. parent and peer attitudes toward active transportation), public policies and institutional practices (i.e. school policies, municipal bylaws), the built environment (i.e. availability and condition of sidewalks and bicycle paths) and the physical environment (i.e. weather). Theoretical models that seek to predict children and youth's travel patterns are typically based on social-ecological theory (Panter et al., 2008; Pont et al., 2011). It is worth noting that one important feature of ecological models is that they posit interactions between these levels of influence (Sallis et al., 2006, 2012). In addition, several correlates have both an objective and a subjective component (Panter et al., 2008); for example long objectively-measured distance between home and school and the perception that the school is too far can both be associated with a reduced likelihood of engaging in AST. The following paragraphs provide a brief overview of the correlates of AST that have been examined in previous research within an ecological perspective. It is noteworthy that some variables could be viewed within different levels of influence because they are interrelated (Bronfenbrenner, 1979; for example, parental road safety concerns could be viewed from both the social environment and the built environment point of view). In this dissertation, individual's perceptions are discussed within the same level of influence as the corresponding objectively-measured characteristics (e.g. parental road safety concerns are discussed in the built environment section).

1.6.1 Personal characteristics. North American research has generally shown that a higher proportion of boys engage in AST (Evenson et al., 2003; Fulton et al., 2005; Gropp et al., 2012; McMillan et al., 2006). However, McDonald (2012) examined gender differences in AST 
in six waves of the US National Household Travel Survey, and she found that the magnitude of these differences varied with travel modes. While the proportion of boys walking to/from school was only slightly higher, cycling to/from school was generally 2-3 times more prevalent in boys.

In North America, studies have consistently reported lower mode shares of AST in secondary school students than in primary school students (Buliung et al., 2009; Evenson et al., 2003; Fulton et al., 2005; Pabayo et al., 2008, 2011). Using prospective data from the Canadian National Longitudinal Survey of Children and Youth, Pabayo and colleagues (2011) reported that the mode share of AST increased with age up to 10 years old and decreased afterwards. Interestingly, data from the 2009 Ontario Student Drug Use and Health Survey indicated that adolescents who had some input to the travel mode decision making process were more likely to walk to/from school (Wong et al., 2011a). Differences in rates of AST between ethnic groups have also been reported in the United States (Evenson et al., 2003; Fulton et al., 2005; Martin et al., 2007).

Cognitive variables such as attitudes, intentions and habits have been shown to predict adults' travel behaviours (Gardner \& Abraham, 2008; Lemieux \& Godin, 2009), but limited research has been done in children and youth. A Scottish study explored the associations of the constructs from Ajzen's (1991) theory of planned behaviour with children's school travel (Murtagh et al., 2012). They found that intention and perceived behavioural control explained $10 \%$ of the variance in the number of steps accumulated during the trip to school, and adding a measure of habit strength to the model increased the proportion of explained variance to $16 \%$. Orsini and O'Brien (2006) interviewed 6 adolescents who were old enough to drive but still chose to cycle to school inquiring about the underlying influences and motivators. The majority of participants mentioned that they cycled because it's fun, it's faster than alternatives and it's 
good for fitness and health. Other studies have reported that children's preference for walking or cycling was a strong correlate of AST (Lee et al., 2013; Trapp et al., 2011).

1.6.2 Social environment. Parents are thought to be the principal decision-maker with respect to their child's travel mode (McMillan, 2005), although children may play a more important role in the decision-making process as they acquire independent mobility (Panter et al., 2008; Pont et al., 2011). In primary school children, independent mobility has been shown to be positively associated with active transportation and PA levels (Mackett et al., 2007; Page et al., 2009), and at a given age, boys generally have greater independent mobility than girls (Prezza et al., 2001; Valentine, 1997).

North American studies have also found that children living in low SES families are more likely to engage in AST (Evenson et al., 2003; Gropp et al., 2012; McDonald, 2008; Pabayo et al., 2008, 2011). Similarly, the likelihood that children engage in AST tends to decrease with increasing parental car ownership (DiGuiseppi et al., 1998; Gropp et al., 2012; Roberts et al., 1997). Social norms (Lee et al., 2013; McMillan et al., 2007) and social support from friends have also been shown to be associated with AST (Hohepa et al., 2007; Hume et al., 2009; Panter et al., 2010a; Timperio et al., 2006). Adolescents who cycled to/from school mentioned the positive influence of parental role modeling (Orsini \& O'Brien, 2006). However, the influence of having siblings on the likelihood of AST is mixed (Ahlport et al., 2008; McMillan, 2007).

Interestingly, while concerns about their child's personal safety (i.e. stranger danger and bullying) are commonly mentioned by parents (Martin \& Carlson, 2005; Timperio et al. 2004), the evidence supporting an association between personal safety concerns and AST is conflicting (Panter et al., 2008). Of note, Timperio et al. (2004) hypothesized that the lack of association 
may have resulted from the high prevalence of parental concerns in both active and inactive travelers in their sample.

Based on semi-structured interviews with 37 parents in the Greater Toronto Area, Faulkner and colleagues (2010) concluded that parental travel mode decisions are essentially based on two key questions: 1) does the child need to be escorted to/from school?; and 2) what is the easiest and most convenient way to travel? Over time, these decisions became a routine, again reflecting the habitual nature of school travel patterns. Quantitative studies have also shown that perceived convenience of driving is a key reason why parents drive their child to school even though they live within walkable distances (Lee et al., 2013; McDonald \& Aalborg, 2009; McMillan, 2007; Panter et al., 2010a; Trapp et al., 2011).

Theoretically, parents could also influence their children's school travel by deciding to live in a neighbourhood that is more or less suitable for AST. For example, parents who value walking and/or PA in general may decide to live in a "walkable" neighbourhood. This might be important because earlier studies have shown that adults who prefer walking and cycling are more likely to choose to live in walkable areas (Frank et al., 2007; Handy et al., 2006). To date, only two cross-sectional studies have explored this issue, and they reported that parents of children who walked to/from school unescorted were more likely to report that they chose to live in their current neighbourhood so that their children could walk to school (Lee et al., 2013; Mammen et al., 2012).

1.6.3 Public policies and institutional practices. Policies may also have an impact on children's travel patterns. For example, in the United States, the mode share of AST is higher in states that require the presence of crossing guards and lower speed limits around schools; 
moreover, walking school bus schemes are more likely to be implemented in school districts that have strong Safe Routes to School policies (Chriqui, 2012; Turner et al., in press). In contrast, two US studies indicated that school policies that limited AST were an important barrier perceived by parents (Ahlport et al., 2008; Martin \& Carlson, 2005). In London (UK), road casualties among children were halved after the introduction of $20 \mathrm{mph}(32 \mathrm{~km} / \mathrm{h})$ speed limits (Grundy et al., 2009). Implementation of a Safe Routes to School intervention in New York City schools has led to a similar decrease in pedestrian injuries while no changes were observed in the control schools (DiMaggio \& Li, 2013). Such improvements in road safety could encourage parents to allow their child to engage in AST. Indeed, McMillan (2007) reported that speed limits $>30 \mathrm{mph}(48 \mathrm{~km} / \mathrm{h})$ were associated with a significantly lower likelihood of AST.

At the community level, the presence of "eyes on the street" (Jacobs, 1961) and greater social interactions may favour AST, but the evidence remains inconclusive. McMillan (2007) observed that the amount of windows facing the street was positively associated with AST in multivariate models. Another US study indicated that neighbourhood social cohesion was a facilitator of AST, especially for children living less than $1.6 \mathrm{~km}$ away from school (McDonald, 2007a). However, other studies have noted that AST was more common in areas not considered "excellent" to raise a child (Pabayo et al., 2011, 2012a) and in neighbourhoods with high levels of incivilities (Rossen et al., 2011). It is possible that limited travel options are available in low income communities, leading children to walk despite a less favourable context.

A systematic review of 14 intervention studies on AST was recently published (Chillón et al., 2011). These interventions were mostly implemented at the school level in the US, UK and Australia. Ten of these studies reported an increase in active transportation and/or PA, but the magnitude of the observed changes was very heterogeneous and the study quality was rated as 
"weak". Since the publication of this review, an evaluation of school travel plans implemented in 33 elementary schools in New Zealand was published, reporting a 5.9\% average increase in AST (Hinckson et al., 2011). The increase was greater in smaller schools, those in which the proportion of children using AST before the implementation of the school travel plan was higher, and those where the plan had been implemented for a longer period of time. Similarly, a pilot evaluation of the Active and Safe Routes to School program in four Canadian provinces has shown a $2.1 \%$ increase in the mode share of active transportation (Buliung et al., 2011).

1.6.4 Built environment. A recent systematic review of studies that measured environmental correlates of AST with geographic information systems has shown that distance between home and school was the only consistent correlate (Wong et al., 2011b). The perception that the school is too far is also consistently associated with lower odds of AST (Lee et al., 2013; Martin \& Carlson, 2005; Nelson et al., 2008; Stewart et al., 2011, 2012). Because secondary schools generally have much larger catchment areas (Environmental Protection Agency, 2003), distance may be a key reason why North American studies have repeatedly shown a lower mode share of AST in secondary school students.

Wong et al. (2011) found conflicting evidence regarding the association between features such as density, land-use mix and street connectivity which are strong correlates of active transportation in adults (Cervero \& Kockelman, 1997; Saelens \& Handy, 2008). Nevertheless, McDonald (2008) observed that the direct influence of density on AST was very small, but that density was strongly correlated with distance which, in turn, was highly correlated with AST. She suggested that in denser areas, it may be more feasible to have a viable school population living within a short distance. In addition, Giles-Corti et al. (2009) argued that the relative 
influence of the built environment on children's AST and PA levels is likely greater in adolescents than in children because the former generally have greater independent mobility.

Further, many studies have consistently found higher rates of AST in urban areas (Mitra et al., 2010; Pabayo et al., 2008, 2011; Panter et al., 2010a); moreover, a study in Halifax (Nova Scotia) found that the contribution of active travel to daily MVPA was greater in adolescents living in urban and suburban areas compared to rural areas (Rainham et al., 2012). However, within Canadian census metropolitan areas, Seliske et al. (2012) observed that adolescents aged 12-15 years were more likely to accumulate at least 30 minutes of active transportation per day in areas with larger urban sprawl, while no differences were observed among older adolescents. Whether this difference was attributable to schools being located further away in more sprawling areas was not assessed.

Parental road safety concerns are generally associated with a higher likelihood of inactive travel (Carver et al., 2008; Kerr et al., 2006; Lee et al., 2013; Panter et al., 2010a; Voorhees et al., 2010), although some studies have found no relationships (Carson et al., 2010; Timperio et al., 2004). In contrast, the presence of sidewalks and cycle paths has been shown to be a facilitator of AST in several studies (Boarnet et al., 2005; Fulton et al., 2005; Kerr et al., 2006), although other studies found no association (Evenson et al., 2006; Mota et al., 2007). Still, Evenson et al. (2006) noted that girls who reported that there are bicycle/walking trails in their neighbourhood were more likely to walk or cycle to school.

1.6.5 Physical environment. Although bad weather conditions are often reported as a barrier to AST (Ahlport et al., 2008; Dellinger \& Staunton, 2002; Martin \& Carlson, 2005), few studies have evaluated the association between objectively-measured weather conditions and 
children and youth's travel mode. Gropp et al. (2012) examined different climate-related variables in a very large sample of Canadians who participated in the 2009-2010 Health Behavior in School-Aged Children survey $(\mathrm{N}=3,997)$ and found no association at the $p<0.05$ threshold.

With respect to seasonal variations, a study in the Greater Toronto Area found no evidence of changes in the proportion of children walking to/from school. Moreover, weakly weather conditions had little impact on mode choice (Mitra \& Faulkner, 2012). The authors argued that, at most, extreme weather event (i.e. snow storms) could trigger short term changes from walking to motorized travel. However, there were too few cyclists to assess whether there were seasonal variations in cycling. A Norwegian study did report a large decrease in cycling during the winter, but most cyclists switched to walking (Børrestad et al., 2011).

Few studies have assessed relationships between topography and AST. Timperio et al. (2006) reported that the presence of a steep incline on the route to/from school was associated with a lower likelihood of AST in children aged 5-6 years, but not in 10-12 year old youth. A qualitative study suggested that hills were an important barrier for cycling; children reported that hills "test their biking skills" (Ahlport et al., 2008).

\subsection{Knowledge gaps.}

In their systematic review, Faulkner and colleagues (2009) noted that the measurement of AST has been inconsistent across studies. Moreover, all included studies have used categorical measures of AST (i.e. active vs. inactive travel) that ignore differences between individuals in distance traveled (or duration of school trips). Two recent studies have shown that distance between home and school can moderate the relationship between AST and PA levels (Panter et 
al., 2011; van Sluijs et al., 2009). Thus, improvement in the measurement of AST is needed for future studies to assess the relationships between AST and health-related outcomes more accurately and to enable the assessment of potential dose-response associations.

In addition, there remains several research gaps with respect to the associations of AST with PA levels and health-related outcomes. First, previous systematic reviews identified no intervention studies that met their inclusion criteria. Because findings from a few interventions studies have recently been published (i.e. Heelan et al., 2009; Mendoza et al., 2011), there is a need to update earlier systematic reviews. Second, a rigorous assessment of the quality of existing evidence remains to be done. Of the three previous systematic reviews (Faulkner et al., 2009; Lee et al., 2008; Lubans et al., 2011), only the latter assessed study quality in a systematic way; this is an important limitation because it places cross-sectional studies on the same level as more rigorous study designs (i.e. controlled trials and prospective studies). Third, although the majority of studies using objective measures of PA have shown that active travelers were more active (Faulkner et al., 2009), previous reviews have not explored whether studies using subjective measures (i.e. questionnaires and diaries) have shown a similar association. Fourth, because the majority of studies that examined the relationship between active transportation and PA levels have focussed solely on the trip to/from school (Panter et al., 2008), the overall contribution of active transportation has likely been underestimated. Fifth, most studies have used cross-sectional designs which cannot determine the direction of observed relationships; hence, it is unclear whether being an active child leads to the decision of walking or cycling to/from school or if AST leads children to become more active (Faulkner et al., 2009). Some researchers have observed that the differences in PA between groups were present only during 
weekdays (Cooper et al., 2003, 2005; Sirard et al., 2005), providing preliminary evidence suggesting that AST may lead children to be more active, but more robust evidence is needed.

Important research gaps also remain with respect to the study of correlates of AST. First, the limitation of cross-sectional designs also applies to the study of correlates of AST. Therefore, the extent to which such correlates vary over time remains unknown (Panter et al., 2008; Pont et al., 2011). In particular, there is a clear need to examine differences in the correlates of AST before and after the school transition given that North American studies have consistently reported lower mode shares of AST in adolescents compared to children (Buliung et al., 2009; Evenson et al., 2003; Fulton et al., 2005; Pabayo et al., 2008, 2011). Second, although children may have some input into travel mode decisions, research assessing the concordance between child- and parent-perceived barriers to AST is scarce (Napier et al., 2011). Third, limited research has examined whether parental neighbourhood selection factors - that is, reasons why parents chose to live in their neighbourhood - can influence their children's school travel patterns. Fourth, few studies have assessed the relationship between composite measures of neighbourhood walkability (e.g. the ability to walk to various destinations within the neighbourhood) and AST and their findings are inconclusive (Wong et al., 2011).

\subsection{Objectives and research questions.}

Based on this literature review, the present dissertation focussed on two overarching objectives: 1) to measure the relationships of AST with PA and health-related outcomes; 2) to examine the correlates of AST immediately before and after the transition from primary to secondary school (hereafter referred to as the "school transition"). The conceptual framework in Figure 1 illustrates the integration of the overarching objectives. In this regard, a large range of 
potential correlates of AST from different levels of influence were examined; hence the illustration of a simplified ecological model. As postulated by other researchers (e.g. Panter et al., 2008; Pont et al., 2011), travel mode decisions are primarily taken based on the parents' perception of the correlates of AST, but children may acquire a greater influence in the decisionmaking process as they get older. In turn, travel patterns may have direct and indirect influences on PA and health-related outcomes.

The current dissertation consists of six studies and Table 1 illustrates how they relate to the two overarching objectives, and briefly summarizes the research questions examined and the methodology employed. Study 1 is a systematic review which aimed to: 1) update current knowledge on the association between AST and daily PA level; 2) examine the associations of AST with indicators of body composition and cardiovascular fitness in children and youth (e.g. 5-18 years old); and 3) systematically assess the quality of evidence with respect to the relationships of AST with daily PA, body composition, and cardiovascular fitness.

The objective of study 2 was to assess the relationships of AST with pedometerdetermined PA, BMI and waist circumference using data from grades 4 to 6 children who participated in cycle 2 of the Canadian Assessment of Physical Literacy study in 2009-2010. It is worth noting that only a small number of studies had previously assessed the association between AST and waist circumference.

Study 3 aimed to assess the relationships of active transportation for a broad range of destinations with objective measures of PA, body composition, physical fitness and CVD risk factors in a large sample of 12- to 19-years-old adolescents who participated in the nationallyrepresentative 2007-2009 Canadian Health Measures Survey. Participants were asked to indicate 
the amount of time they spent walking and cycling for utilitarian purposes (e.g. to/from school, work and while doing errands) in a typical week during the past 3 months.

Studies 4, 5 and 6 present different aspects of a prospective pilot-study ${ }^{2}$ that focussed on the transition from primary to secondary school. The survey instruments are provided in Appendices 6-9. Specifically, manuscript 4 aimed to measure the changes in AST and pedometry-measured PA levels across the school transition. This study also aimed to evaluate the test-retest reliability of a novel method to calculate the volume of $\mathrm{AST}^{3}$ by combining the frequency of active trips to/from school and the distance between participant's residence and the school that they attend.

Manuscript 5 had two key objectives: 1) to evaluate the concordance of child- and parentperceived barriers to AST; 2) to assess how perceived barriers to AST, parental neighbourhood selection factors and different measures of household SES are associated with individuals' primary travel mode. These analyses were performed both before and after the school transition.

Finally, the objective of manuscript 6 was to examine the association of neighbourhood walkability with AST and pedometer-determined PA levels at both time points. The Walk Score ${ }^{\circledR}$ application ( $\underline{\text { www.walkscore.com }}$ ) was used as a measure of walkability given that previous research has found strong correlations between Walk Scores and objective measures of the built environment such as residential density, intersection density, street density, average block length and access to public transit (Carr et al., 2010). Walkability was assessed both around the home and around the schools and analyses were performed at both time points.

\footnotetext{
${ }^{2}$ Given the large amount of study objectives (see Table 1), the findings are reported in three distinct manuscripts.

${ }^{3}$ The volume of AST was calculated with the following equation: volume $=\ln (1+$ frequency $X$ distance $)$ with the constant (1) added to ensure that no volume was below 0. Distance between home and school was skewed, hence the log-transformation.
} 

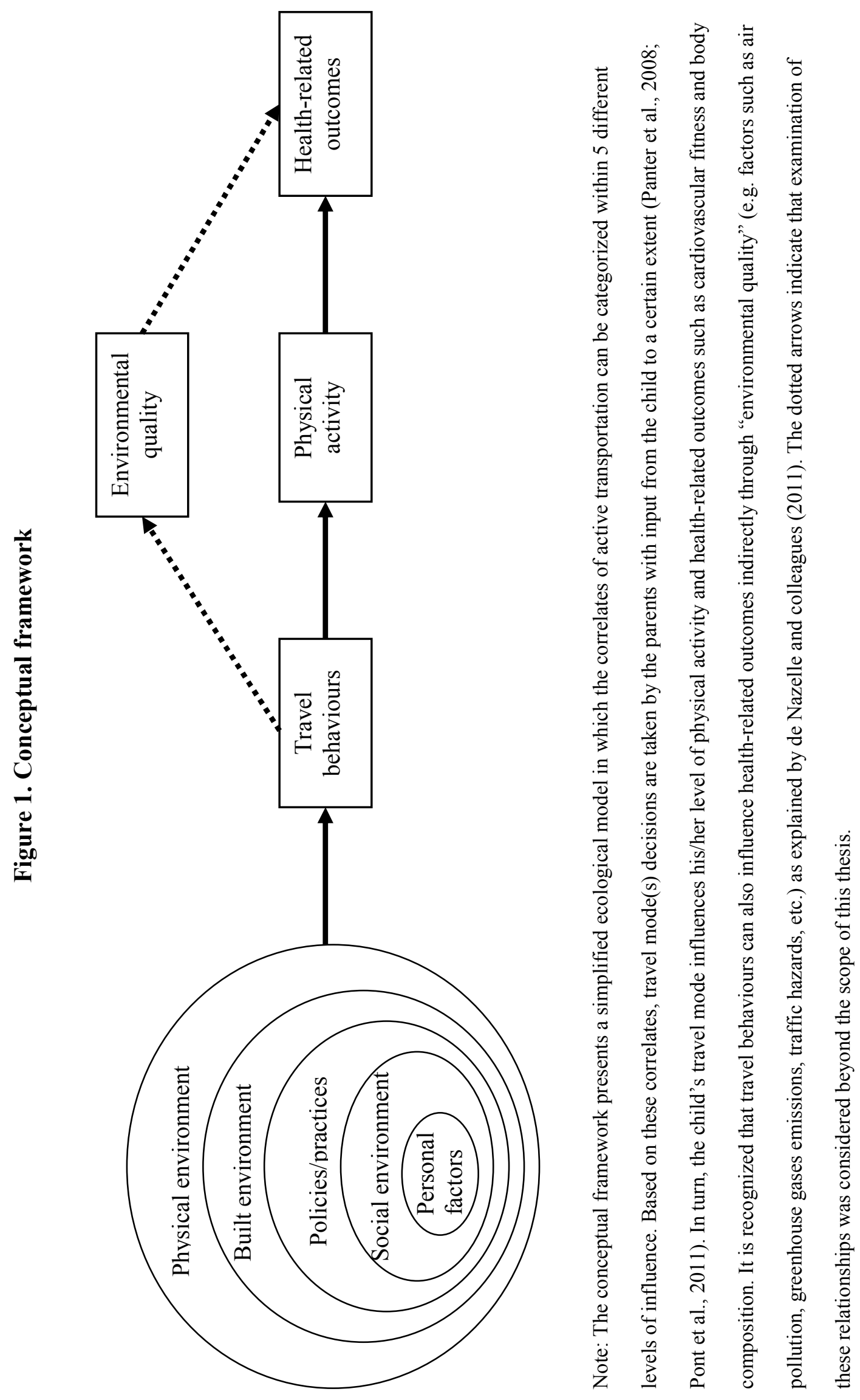


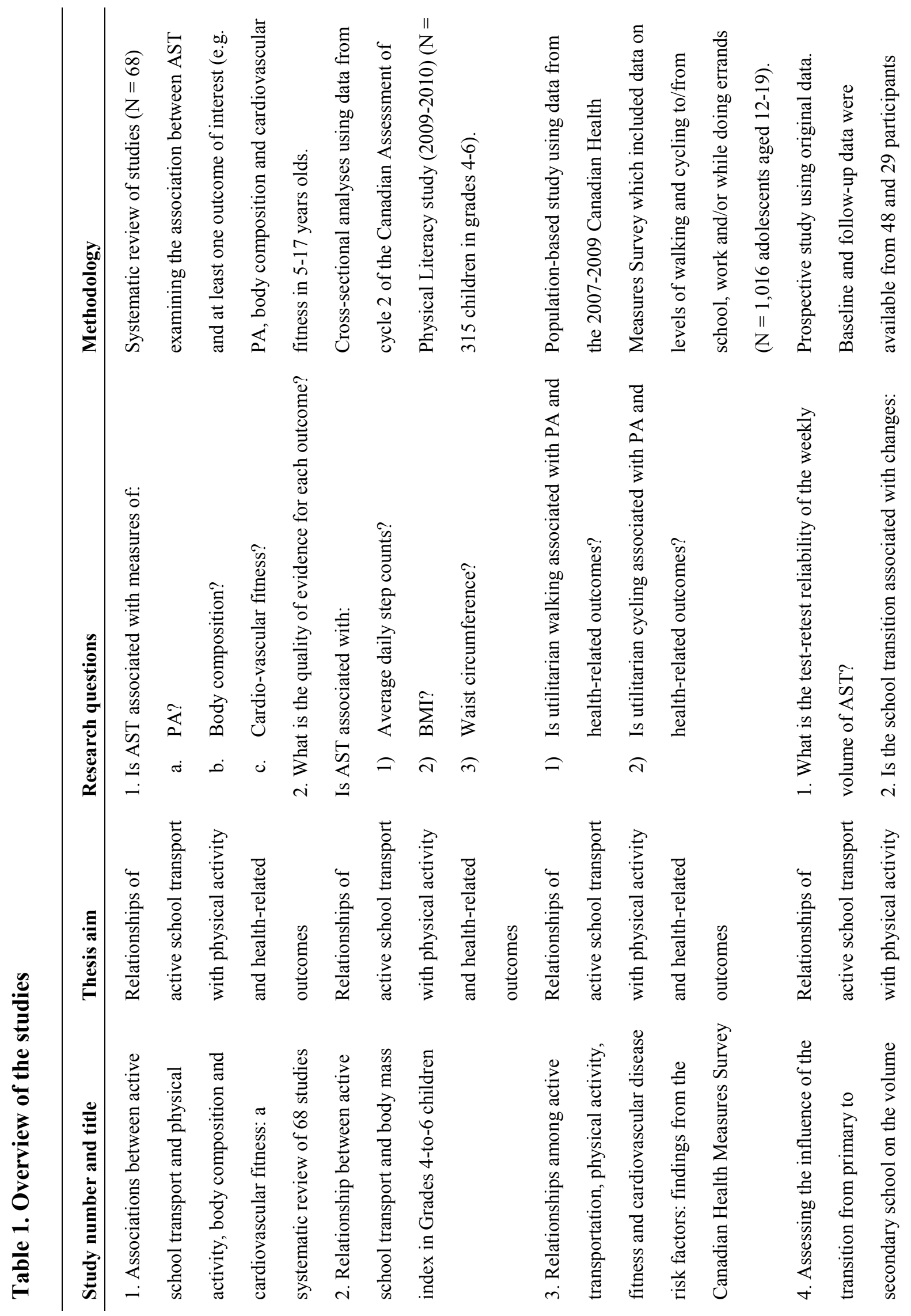




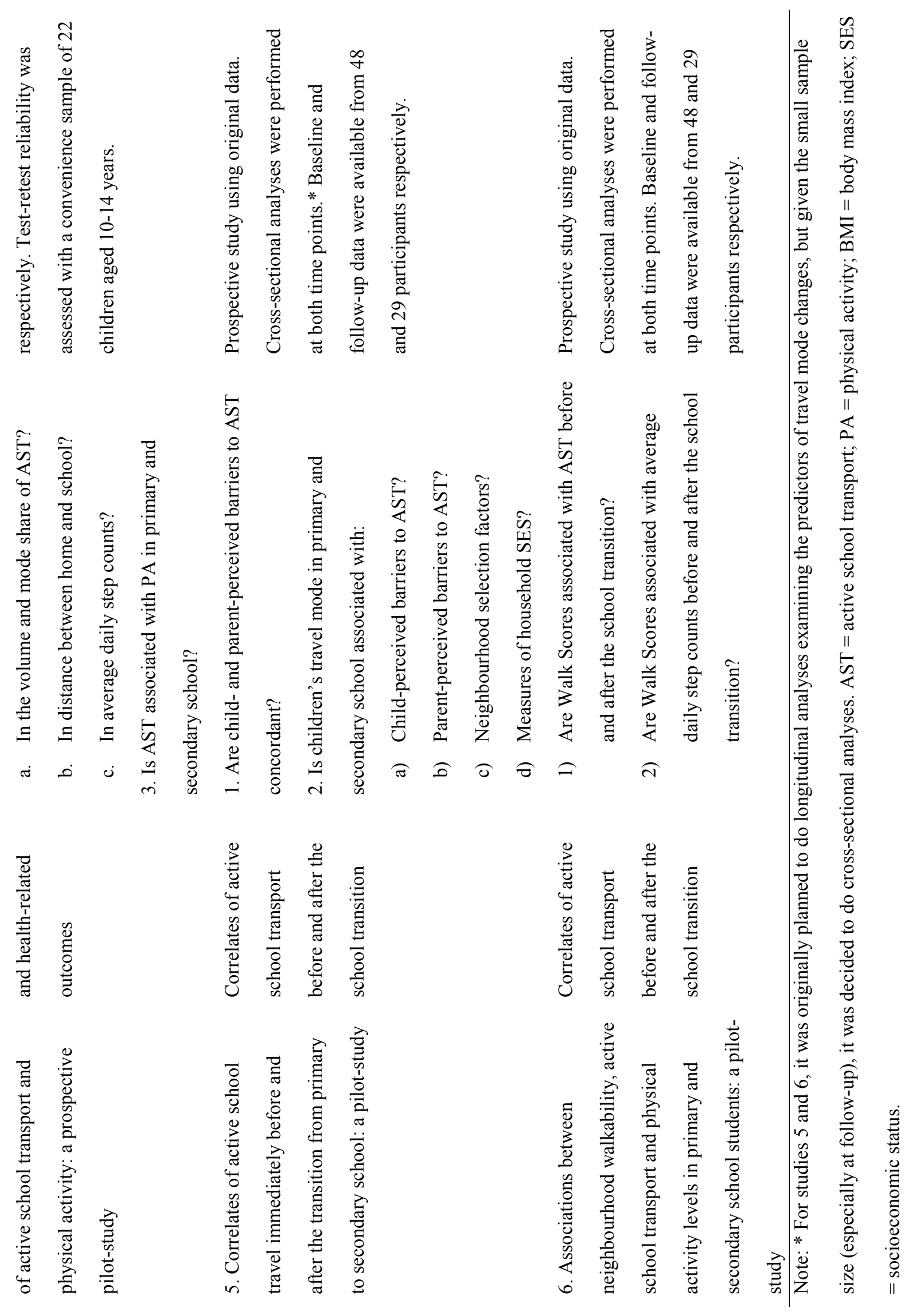

ส 


\subsection{Organization of the thesis.}

The first four studies (Chapters 2 to 5 ) focussed on overarching objective 1 while the last two studies (Chapters 6 to 7) focussed on overarching objective 2. The general discussion is also organized according to these major objectives. Hence, the findings of Chapters 2 to 5 (which focussed predominantly on the associations between active transportation and health-related outcomes) are discussed first. Then the findings of Chapters 6 and 7 which examined correlates of AST are discussed. Then, the limitations and strengths of the studies are critically evaluated. Finally, the implications of the six studies for future research and interventions are discussed. 
Chapter 2. Study I: “Associations between active school transport and physical activity, body composition and cardiovascular fitness: a systematic review of 68 studies"

Authors: Richard Larouche, Travis Saunders, Guy E.J. Faulkner, Rachel C. Colley, Mark S. Tremblay

Status: accepted for publication in Journal of Physical Activity and Health on June 13, 2012.

Presented at: International Conference on Enhancement of Physical Activity. Vuokatti, Finland (2011). 
-Associations Bctween Active Scheol Transport and Physical Activity, Body Composition and Cardiovascular Fitness: A Systematic Review of 68 Studies" by Larouche R et al.

Jowrnal of Physical Activity \& Heath

O 2012 Human Kinetics, Inc.

\section{Note: This article will be published in a forthcoming issue of the Journal of Physical Activity \& Health. This article appears here in its accepted, peer-reviewed form, as it was provided by the submitting author. It has not been copy edited, proofed, or formatted by the publisher.}

Section: Review

Article Title: Associations Between Active School Transport and Physical Activity, Body Composition and Cardiovascular Fitness: A Systematic Review of 68 Studies

Authors: Richard Larouche', Travis John Saunders', Guy Edward John Faulkner ${ }^{2}$, Rachel Colley', and Mark Tremblay'

Affiliations: ${ }^{1}$ Healthy Active Living and Obesity Research Group, Children's Hospital of Eastem Ontario, Ottawa, Ontario, Canada. ${ }^{2}$ Faculty of Physical Education and Health, University of Toronto, Toronto, Ontario, Canada.

Running Head: Active school transport and health

Journal: Journal of Physical Activity \& Health

Acceptance Date: June 13,2012

(0) 2012 Human Kinetics, Inc. 
"Associations Bctween Active School Transport and Physical Activity. Body Composition and Cardiovascular Fitneas: $A$ Systematic Review of 68 Studies" by Laroucbe R et al.

Jownal of Physical Activity \& Feaith

6. 2012 Human Kinetics, Inc.

Title: Associations between active school transport and physical activity, body composition and cardiovascular fitness: a systematic review of 68 studies

Manuscript type: Review

Running Head: Active school transport and health

Word count: 5750

Abstract word count: 200

Date of submission: October 12,2011

Keywords: Active travel, exercise, body weight, cardiorespiratory fitness, youth 
-Associations Between Active School Transport and Physical Activity, Body Composition and Cardiovascular Fitnessi A Syslematic Review of 68 . Studies" by Larouche R ef al.

Jownal of Physical Activity \& Health

C 2012 luman Kinctics, Inc.

Abstract:

Background: The impact of active school transport (AST) on daily physical activity (PA) levels, body composition and cardiovascular fitness remains unclear. Methods: A systematic review was conducted to examine differences in PA, body composition and cardiovascular fitness between active and passive travelers. The Medline, PubMed, Embase, Psyclnfo and ProQuest databases were searched and 10 key informants were consulted. Quality of evidence was assessed with GRADE and with the Effective Public Health Practice Project tool for quantitative studies. Results: 68 different studies met the inclusion criteria. The majority of studies found that active school travelers were more active or that AST interventions lead to increases in PA, and the quality of evidenee is moderate. There is conflicting, and therefore very low quality evidence, regarding the associations between AST and body composition indicators, and between walking to/from school and cardiovascular fitness. However, all studies with relevant measures found a positive association between cycling to from school and cardiovascular fitness; this evidenee is of moderate quality, Conclusion: These findings suggest that AST should be promoted to increase PA levels in children and adolescents and that cycling to from school is associated with increased cardiovascular fitness. Intervention studies are needed to increase the quality of evidence.

Keywords: Active travel, exercise, body weight, cardiorespiratory fitness, youth 
"Associations Between Active Sehool Transport mod Physical Activity, Body Compositios and Cardiovascular Fitness: A Systematic Review of 68 Studies" by Larouche $R$ a al.

Journal of Physical Activity \& Headth

c. 2012 lluman Kinetis, Ine.

\section{Introduction}

According to recent physical activity guidelines, children and adolescents should accumulate at least 60 minutes of moderate- to vigorous-intensity physical activity (MVPA) every day, ${ }^{1,2}$ However, population data from the Canadian Health Measures Survey and the National Health and Nutrition Examination Survey indicate that the majority of children and adolescents fail to meet this threshold. ${ }^{3,4}$ Researchers are increasingly referring to this situation as a tphysical inactivity crisis,, 5 because low physical activity (PA) has been linked to a higher prevalence of obesity and to the emergence of cardiovascular disense risk factors in childhood and adolescence. ${ }^{6-8}$ Moreover, the rising prevalence of obesity has coincided with a significant decline in fitness among children and youth over the past few decades, 90

Active school transport (AST) has been proposed as a way to increase PA in children and youth at the population level ${ }^{11}$ with the added benefit of reducing emissions of greenhouse gases and other pollutants. ${ }^{12-14}$ It is an inexpensive form of physical activity that can be integrated into individuals ${ }^{+}$routines as most children and adolescents must travel to and from school on a daily basis. In addition, it has been argued that if sufficient intensity is achieved, AST could lead to an increase in cardiovascular fitness. ${ }^{15}$ However, in recent years, the prevalence of AST has declined in several countries including Canada, USA, Australia and Switzerland: ${ }^{16-19}$

Two systematic reviews published in 2008 and 2009 have examined the relationship between AST, PA level and body weight, ${ }^{20,21}$ and both concluded that AST was positively associated with PA level in the majority of studies. However, there were no intervention studies and very few longitudinal studies; hence, it was impossible to determine the direction of this relationship. In addition, most of the studies included in these reviews did not show an association between AST and body weight. 
"Associations Between Active School Transport and Physical Activity, Body Composition and Cardiovascular Fitness: A Systematic Review of 68 Studies" by Larouche R et al.

Joumal of Physical Activity \& Health

2012 Human Kinetics, Inc.

In contrast with the two earlier reviews described above, a more recent review by Lubans and colleagues has reported that nearly half of available studies have shown that AST was associated with a more favorable body composition. ${ }^{22}$ The majority of studies also indicated that active travelers had greater cardiovascular fitness, especially those who cycled to and from school. However, their review did not examine differences in PA between active and passive travelers. Of the three previous systematic reviews, only Lubans et al. assessed study quality. Specifically, they evaluated the quality of reporting and calculated "levels of evidence" based on the proportion of studies showing significant associations. An important limitation of this approach is that it places cross-sectional studies on the same level as more rigorous study designs (i.e. controlled trials and prospective studies). Finally, the three earlier reviews have identified only observational studies.

Before broader funding and implementation of strategies to promote AST can be realized, it is important to demonstrate that AST is associated with positive health-related outcomes. Consequently, the main objectives of the current systematic review were: 1) To update current knowledge on the association between AST and daily PA level;2) To examine the association between AST, cardiovascular fitness and various indicators of body composition (BMI, waist circumference, skinfolds, etc.); and 3) To systematically assess the quality of evidence with respect to the relationship between AST and daily PA, cardiovascular fitness, and body composition.

\section{Methods}

Search strategy. A systematic search strategy was developed with the help of a professional librarian and captured a total of 2396 potentially relevant articles published (or in press) prior to April 10,2011. First, the Medline, Embase and PsycInfo databases without date 
"Associations Between Active School Transport and Physical Activity, Body Composition and Cardiovascular Fitness: A Systematic Review of 68 Studies" by Larouche R et al.

Joumal of Physical Activity \& Health

Q 2012 Human Kinetics, Inc.

limits were scanned with the OVID interface using the following search keywords: transport* or commut* or travel* or walk* or cycl* or skat* or skiing or run* or canoe*. At least one of these keywords had to be located within the 3 adjacent words of the word "school". Second, the ProQuest Dissertation \& Theses database was searched for relevant grey literature. Third, the PubMed related articles function was used with the 3 newest and 3 largest studies included in Faulkner and colleagues' review ${ }^{20}$ as seed articles. Date was limited to records indexed in PubMed since May 2008, just prior to the search of the original review. In addition, 10 leading AST researchers, representing 7 different countries (Denmark, Netherlands, Germany, Canada, United States, Australia and United Kingdom), were asked to provide a list of 5 key articles. All of them kindly responded to our query and suggested 23 different papers and reports. Only 5 of them were not captured by the initial search strategy. Finally, 15 articles were added from the authors' personal libraries. Figure 1 illustrates the number of papers retrieved from each of these sources.

The potentially relevant articles were imported into a Reference Manager 11 database (Thompson Reuters, San Francisco, CA) and titles and abstracts were screened independently by two reviewers (RL and TJS). Full text copies of all articles that passed the initial screening were examined by two reviewers (RL and TJS) for inclusion criteria. Any disagreement was resolved with the help of a third reviewer (MT). Consensus was reached for all included articles. The review methodology was prospectively registered in PROSPERO (Registration number: CRD42011001456).

Inclusion and exclusion criteria. To be included in the review, studies had to report the findings of an original study with school-aged children (5.0 to 17.9 years old) and to have assessed at least one of the following outcomes: 1) Daily PA level (objective or subjective 
"Associations Between Active School Transport and Physical Activity, Body Composition and Cardiovascular Fitness: A Systematic Review of 68 Studies" by Larouche R et al.

Joumal of Physical Activity \& Health

2012 Human Kinetics, Inc.

measures); 2) Body composition variables (BMI, waist circumference, skinfolds, etc.); 3)

Cardiovascular fitness (i.e. $\mathrm{VO}_{2} \mathrm{max}$ ). Literature reviews and studies where the mean age of participants was outside the specified range were excluded. Studies of correlates of AST (e.g. in which AST was studied as the outcome variable rather than as an exposure) were excluded. Papers in languages other than English and French were also excluded.

Data extraction. Standardized data extraction tables were created with Microsoft Excel. Information relative to the study design (year, country, participants, age, and methodology), assessment of AST, outcomes examined and the main findings were extracted. The reviewers were not blinded to the authors or journals when extracting data. Authors were contacted for missing information on outcome variables and all of them responded. When the findings of dissertation theses were later published in peer-reviewed journals, data from the latter publication was extracted, unless unavailable. Similarly, when the findings of the same study were discussed in different papers, the data was extracted from the paper that described the differences between active and passive travelers more thoroughly. Following data extraction, it was determined that the included studies were very heterogeneous with respect to the categorization of participants (some studies analyzed walkers and cyclists separately, others pooled them together; also some studies classified individuals using AST only once per week as "active commuters"). Moreover, although pedometers, accelerometers and questionnaires can all provide information about daily PA, they measure quite different constructs (steps per day, minutes spent at a certain intensity and one's perceived level of PA), and these outputs are difficult to compare. ${ }^{23,24}$ The accelerometry studies have also used various models of accelerometers and various intensity cut-points. Therefore, it was considered inappropriate to perform meta-analyses; hence, a qualitative synthesis of the evidence is presented. 
"Associations Between Active School Transport and Physical Activity, Body Composition and Cardiovascular Fitness: A Systematic Review of 68 Studies" by Larouche R et al.

Joumal of Physical Activity \& Health

2012 Human Kinetics, Inc.

Quality of evidence. The quality of the included studies was systematically evaluated using the Effective Public Health Practice Project Quality Assessment Tool for Quantitative Studies. ${ }^{25}$ This tool includes six components: 1) the likelihood that the study participants are representative of the target population;2) the study design; 3 ) the control of confounding variables; 4) the blinding of participants and investigators; 5) the validity and reliability of the data collection tools; and 6) the proportion of withdrawals and drop-outs. This tool was used for each study separately and each component was rated as "strong", "moderate" or "weak" based on standard criteria. ${ }^{25}$ Overall quality ratings were derived from the component ratings as follows: "strong" when there were no weak component ratings, "moderate" when there was one weak component rating and "weak" when there was at least two weak component ratings.

In line with an earlier review of the health-related impacts of cycling ${ }^{26}$, the representativeness component was considered not applicable for experimental studies. Acknowledging that external validity is a very common limitation of experimental studies, if the representativeness component was rated as weak, experimental studies would be extremely unlikely to provide strong evidence based on the Effective Public Health Practice Project tool. The blinding component was also considered non-applicable because it is virtually impossible to blind participants that they are receiving an AST intervention. In addition, none of the included studies described the blinding of participants and/or investigators. The quality assessment was pilot tested independently by two reviewers (RL and TJS) for five studies. The reviewers then discussed any ratings where there was a disagreement in order to reach a consensus. The remainder of the studies was assessed by one reviewer (RL). This procedure was previously employed by de Bourdeaudhuij and colleagues. ${ }^{27}$ 
"Associations Between Active School Transport and Physical Activity, Body Composition and Cardiovascular Fitness: A Systematic Review of 68 Studies" by Larouche R et al.

Joumal of Physical Activity \& Health

Q 2012 Human Kinetics, Inc.

The GRADE approach was used to evaluate the quality of evidence. ${ }^{28,29}$ In this approach, randomized controlled trials begin as high quality evidence, but quality may be downgraded based on factors such as limitations of the study design or implementation, imprecision of estimates (i.e. large confidence intervals), variability in results, and indirectness of evidence. Observational studies begin as low quality evidence, but they may be upgraded if there are large effect sizes, a dose-response gradient or if all plausible biases would be expected to reduce the treatment effect. ${ }^{28,29}$ Consensus was reached among the review team for all GRADE ratings.

\section{Results}

After removal of duplicates, 1433 articles were scanned and 1287 of them were rejected on the basis of titles and abstracts. Full text copies of the remaining 144 papers were screened in detail for inclusion criteria. Seventy one papers were rejected for the following reasons:

ineligible outcome (i.e. no relevant outcome data or AST measured as outcome rather than exposure), 30 papers; ineligible exposure (i.e. overall walking and cycling instead of AST), 22 papers; ineligible population (e.g. mean age outside of 5-18 years old age range), 3 papers; language other than English and French, 6 papers; and literature reviews, editorials, letters to the editor and conference abstracts, 13 papers. Some papers were excluded for multiple reasons. Finally, as illustrated in Figure 1, a total of 73 papers (representing 68 individual studies) were included in the review. Of these, 54 studies were cross-sectional, 10 were longitudinal and 4 were intervention studies (two randomized and two non-randomized). This review includes data from 108694 individuals and the sample size of the studies varied from 11 to 7023 participants. Characteristics of the individual studies are presented in Table 1. It is worth noting that studies were only eligible for inclusion in the PA analyses if they assessed total daily PA (e.g. those that examined only sub-categories such as leisure-time PA were excluded from these analyses). Such 
"Associations Between Active School Transport and Physical Activity, Body Composition and Cardiovascular Fitness: A Systematic Review of 68 Studies" by Larouche R et al.

Journal of Physical Activity \& Health

2012 Human Kinetics, Inc.

studies were still included in the review if other relevant outcomes were reported (e.g. body composition, fitness); however the differences in PA were not examined in the analyses.

Physical activity. As detailed in Table 2, 49 studies examined the association between AST and daily PA, including 2 randomized controlled trials, 2 quasi-experimental studies, 5 prospective studies and 41 cross-sectional studies. Of these, 28 assessed PA using accelerometers $^{30-52,91,92,95,99,102}, 9$ using pedometers ${ }^{53-61}$ and 14 using questionnaires or diaries ${ }^{51,}$ 62-72, 92, 93 (two studies used both accelerometers and questionnaires) ${ }^{51,92}$. Children using AST

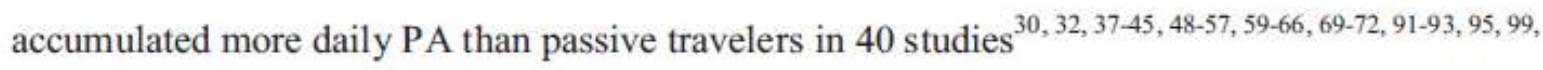
${ }^{102}(81.6 \%$ of the studies), while the 9 remaining studies found no difference between modes of transportation $^{31,33-36,46,58,67,68}$.

AST was associated with higher PA levels in 22 of the 28 studies that used accelerometers $(78.6 \%){ }^{30,32,37-45,48-52,91,92,95,99,102}$ The mean differences in time spent in daily MVPA ranged from 0 to 45 minutes. Two observational studies have shown a dose-response gradient such that active travelers who lived further away from school accumulated more PA than those who lived closer. ${ }^{30,50}$ It is noteworthy that the difference in PA between active and passive travelers often exceeded the amount associated with the journey to and from school. For example, Cooper and colleagues reported that boys who walked to school accumulated 30 additional minutes of MVPA between 3 PM and 8 PM while no such difference was noted in girls. $^{42}$

Both randomized controlled trials have shown significant increases in daily MVPA. Sirard et al. reported an increase of 14 minutes of MVPA during the 45 minute period before school in participants assigned to the walking school bus, with no changes in the control group and no difference between groups for the rest of the day. ${ }^{45}$ Mendoza et al. noted that participants 
"Associations Between Active School Transport and Physical Activity, Body Composition and Cardiovascular Fitness: A Systematic Review of 68 Studies" by Larouche R et al.

Joumal of Physical Activity \& Health

O 2012 Human Kinetics, Inc.

from the experimental group increased their daily MVPA by 2.2 minutes while their control counterparts decreased their daily MVPA by 4.8 minutes. ${ }^{52}$ Similarly, one quasi-experimental study found that participants in the school where walking school buses were implemented for 2 years accumulated about 17.5 more minutes of daily MVPA than children from the control school. ${ }^{44}$ In the other quasi-experimental study, a lower seasonal decrease in daily MVPA and steps was observed in the intervention group (partial $\eta^{2}=0.08$ and 0.09 respectively), but no difference between groups were found during the morning and afternoon school trips. ${ }^{102}$

Eight of the 9 studies that used pedometers found that AST was associated with higher daily step counts $(88.9 \%){ }^{53-57,59-61}$ Mean differences in pedometry-determined PA ranged from 663 to 3477 steps/day. Loucaides and Jago examined differences in step counts in multiple segments of the day and they found that participants who actively traveled to school accumulated 372 more steps in the 'before school' period $\left(\eta^{2}=0.08\right), 1430$ more in the 'after school' period and 1915 more in the whole day $\left(\eta^{2}=0.03\right.$ and 0.04 respectively). ${ }^{54}$

Twelve of the fourteen studies that used self-report instruments have shown that active travelers were more active $(85.7 \%),{ }^{51,62-66,69-72,92,93}$ while two studies reported no difference between groups. ${ }^{67,68}$ Direct comparison of these studies is complicated because some studies used participant reports while others used proxy reports from the parents. Furthermore, differences between groups are reported using different metrics (i.e. daily PA, PA index, proportion of participants meeting PA guidelines, etc.). Both studies that used accelerometers in combination with a questionnaire found that walkers and cyclists were significantly more active with both instruments. ${ }^{51,92}$

Although most studies found that active travelers were more active, some reported that differences in PA were significant only in boys, ${ }^{42,46,48,52}$ while Cooper et al. reported that the 
"Associations Between Active School Transport and Physical Activity, Body Composition and Cardiovascular Fitness: A Systematic Review of 68 Studies" by Larouche R et al.

Journal of Physical Activity \& Health

2012 Human Kinetics, Inc.

difference was significant only in girls and in younger participants. ${ }^{38}$ In contrast, Abbott et al. reported that differences in PA were seen in Year 5 and 10, but not in Year 1 children. ${ }^{53}$ Another study found that girls who walked to school were more active than those who were driven, while boys who cycled to school were more active than passive travelers. ${ }^{50}$ Two accelerometry studies have found differences in PA between walkers and passive travelers, but not between cyclists and passive travelers. ${ }^{38,48}$

Overall, $81.6 \%$ of the studies (including the two randomized controlled trials) have shown that AST was associated with significantly higher PA levels, despite the limitation of accelerometers and pedometers in measuring PA during cycling. ${ }^{24}$ Further, there is some evidence of a dose-response relationship. ${ }^{30,50}$ However, many studies have found inconsistencies of effect between genders or age groups. Therefore, the quality of evidence was rated as moderate.

Body composition. Thirty nine studies examined the association between AST and the following indicators of body composition: BMI ( 38 studies) $33,34,36-39,41-44,46,47,54,58,63,64,67,68,70$,

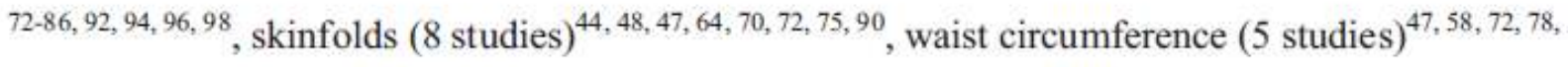
${ }^{90}$, dual-energy X-ray absorptiometry $(2 \text { studies })^{33,36}$, bioimpedence ( 1 study $)^{64}$ and air displacement plethysmography $(1 \text { study })^{35}$. There was 1 quasi-experimental study, 10 prospective studies and 28 cross-sectional studies. Outcomes of these studies are presented in Table 3. Overall, 22 studies (56.4\%) showed no difference between active and passive travellers $^{33-39,41-43,46,54,63,68,72,73,79,82-84,92,94}, 14$ (35.9\%) observed that active travelers had more favorable body composition ${ }^{44,47,58,64,67,75-77,80,81,85,86,96,98}$ and $3(7.7 \%)$ reported that AST was associated with less favorable body composition ${ }^{70,74,78}$. 
"Associations Between Active School Transport and Physical Activity, Body Composition and Cardiovascular Fitness: A Systematic Review of 68 Studies" by Larouche R et al.

Joumal of Physical Activity \& Health

(.) 2012 Human Kinetics, Inc.

Similarly, twenty-two of the 38 studies that assessed BMI found no differences between active and passive travelers $33,34,36-39,41-43,46,54,63,64,68,72,73,79,82-84,92,94,13$ observed that active travelers had lower BMI indices (and/or were less likely to be overweight or obese) $44,47,58,67,75$ $77,80,81,85,86,96,98$ and 3 noted that AST was associated with higher BMI indices ${ }^{70,74,78}$. Heelan et al. showed that participants who walked at least $50 \%$ of the time had significantly lower increases in BMI, body fat percentage and sum of triceps and calf skinfolds than those who walked less than $50 \%$ of the time and passive travelers. ${ }^{44}$ However, 7 of the 10 longitudinal studies reported no difference in BMI between active and passive travelers. ${ }^{33,34,36,46,82-84}$ Of these, 2 papers presented data from the same study, but with different lengths of follow-up ${ }^{33,34}, 1$ study consisted only of a 10-week follow-up period ${ }^{84}$, and another study reported differences between groups at baseline but not at follow-up ${ }^{46}$. One study found a small but positive correlation $(\mathrm{r}=0.03)$ between AST and BMI at follow-up, although additional analyses indicated that this difference was only present in the overweight participants. ${ }^{70}$ In contrast, participants from the Québec Longitudinal Study of Child Development who consistently used AST from kindergarten to Grade 2 had significantly lower BMI indices than passive travelers in Grades 1 and 2, despite a lack of difference in kindergarten. ${ }^{81}$ Similarly, Bere and colleagues ${ }^{86}$ reported that compared to individuals who did not cycle at baseline and/or follow-up, participants who cycled at both time points had a significantly lower risk of being overweight at follow-up $(\mathrm{OR}=$ 0.44) while those who stopped cycling during the follow-up period had an elevated risk ( $\mathrm{OR}=$ 3.19).

Four out of 8 studies that examined differences in skinfold thickness found that active travelers were leaner ${ }^{44,47,64,75}$ while the remaining 3 studies showed no difference between groups $\mathrm{s}^{46,70,72,90}$. Of the 5 studies that examined differences in waist circumference, one found a 
"Associations Between Active School Transport and Physical Activity, Body Composition and Cardiovascular Fitness: A Systematic Review of 68 Studies" by Larouche R et al.

Journal of Physical Activity \& Health

2012 Human Kinetics, Inc.

positive association ${ }^{78}$, while the 4 others indicated that although active travelers had lower waist circumferences, the differences were not significant. ${ }^{47,58,72,90}$ The study that used bio-impedence reported that active travelers had lower percentages of body fat when adjusting for the distance between home and school. ${ }^{64}$ Finally, studies that used dual-energy X-ray absorptiometry or air displacement plethysmography (e.g. BodPod) found no difference between active and passive travelers. $^{33,35,36}$

While $35.9 \%$ of the studies have shown that AST was associated with more favorable body composition, the findings are very inconsistent. In addition, the lack of summary data in several included papers may suggest selective reporting. Therefore, the quality of evidence was rated as very low.

Cardiovascular fitness. Ten studies examined the impact of AST on cardiovascular fitness $^{34,38,48,63,68,72,74,87,94,97}$ (see Table 4). Of these, 4 studies used a cycle ergometer protocol $^{34,38,48,68}, 4$ used the $20 \mathrm{~m}$ shuttle run test ${ }^{63,72,94,97}$ and 2 used the 1 -mile run time. ${ }^{74,87}$ Of note, Andersen and colleagues directly measured expired gases on a subsample of 287 participants to determine whether differences in $\mathrm{VO}_{2}$ max were due to a better mechanical efficiency in cyclists. ${ }^{68}$ Their results show that differences in work capacity were not an artifact of greater mechanical efficiency.

The findings differ markedly between walking and cycling. All 5 studies that assessed walking and cycling separately as recommended by Shephard ${ }^{15}$ found that cyclists had greater cardiovascular fitness than passive travelers. ${ }^{34,38,48,63,68}$ Data from the 6-year follow up of the Danish arm of the European Youth Heart Study has shown that children who started cycling during the follow-up period achieved a $9 \%$ greater cardiovascular fitness at 15 years of age, and cycling to school explained $3.1-9.7 \%$ of the variance in cardiovascular fitness. ${ }^{34}$ In addition, 
"Associations Between Active School Transport and Physical Activity, Body Composition and Cardiovascular Fitness: A Systematic Review of 68 Studies" by Larouche R et al.

Joumal of Physical Activity \& Health

Q 2012 Human Kinetics, Inc.

findings from the Danish youth and sport study indicate that adolescent cyclists had higher maximal aerobic power (4.6-5.9\%), isometric abdominal endurance (10-16\%), dynamic abdominal endurance $(10 \%)$, and trunk flexibility $(6 \%) .{ }^{68}$ However, only the largest study in which walkers and cyclists were analyzed separately $(\mathrm{N}=6085)$ observed that walkers were fitter than passive travelers. ${ }^{63}$

For the five remaining studies, cyclists were combined with walkers in the analyses due to the scarcity of cycling. ${ }^{72,74,87,94,97}$ Madsen and colleagues reported that although active travelers achieved better mile run times, the difference was not significant $(p=0.07 ; \beta=-2.0$; $95 \% \mathrm{CI}=-4.2$ to 0.2$).{ }^{74}$ In contrast, Heelan and colleagues reported a significant correlation $(\mathrm{r}=$ 0.34 ) between mile times and the frequency of AST among children who actively traveled 0.5 to 1 mile, but not in those who travelled shorter distances. ${ }^{87}$ Three studies found that active travelers were more likely to be classified as "fit" based on their performance on the $20 \mathrm{~m}$ shuttle run test $\mathrm{t}^{72,94,97}$, but one of these noted that significant differences were observed in only one of the two cohorts. ${ }^{94}$

Considering that all 5 studies found that cyclists had significantly better cardiovascular fitness than passive travelers, and that controlling for PA in the analyses may bias the results toward the null hypothesis (e.g. co-linearity between AST and PA), the current evidence showing higher cardiovascular fitness in children who cycle to/from school is rated as moderate.

However, there is currently inconclusive evidence that walking to school is associated with higher cardiovascular fitness due to the variability in study findings. It is therefore concluded that this evidence is of very low quality.

Quality assessment. Based on the Effective Public Health Practice Project quality assessment tool, 21 studies were rated as weak, ${ }^{39,40,42,49,50,57,59-61,64-67,72,74,76,84,91,93,95,98} 45$ as 
"Associations Between Active School Transport and Physical Activity, Body Composition and Cardiovascular Fitness: A Systematic Review of 68 Studies" by Larouche R et al.

Journal of Physical Activity \& Health

( 2012 Human Kinetics, Inc.

moderate, ${ }^{30-33,35-38,41,43-48,51,53-56,58,62,63,68-71,73,75,77-83,85,86,92,94,96,97,99,100,102}$ and 2 as strong ${ }^{34,}$

${ }^{52}$. Ratings for each component of the tool are provided in Table 5. Overall, a cross-sectional

study design was the most common reason for studies to be rated as weak.

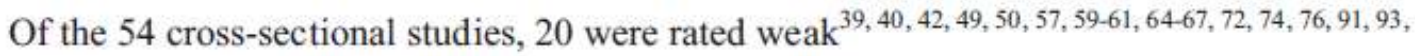
95,98 and 34 were moderate ${ }^{30-32,35,37,38,41,43,47,48,51,53-56,58,62,63,68,69,71,73,75,77-80,85,92,94,96,97,99,}$

${ }^{100}$. Cross-sectional studies cannot be rated as strong based on the Effective Public Health

Practice Project tool because they are automatically assigned a weak rating for study design.

Eight out of ten longitudinal studies were rated as moderate $\mathrm{e}^{33,36,46,70,81-83,86}$, one as weak ${ }^{84}$ and

one as strong ${ }^{34}$. Three of the four intervention studies were rated as moderate. The quasi-

experimental study by Heelan et al. had a very high drop-out rate ${ }^{44}$. The randomized controlled

trial by Sirard et al. did not control for potential confounders, likely because of the low sample

size $(\mathrm{N}=11)^{45}$. McMinn et al reported an important seasonal effect and concerns regarding the

fidelity of the intervention implementation. However, Mendoza and colleagues' randomized

controlled trial was rated as strong. ${ }^{52}$

\section{Discussion}

The primary objective of the current systematic review was to examine the impact of AST on PA levels, indicators of body composition and cardiovascular fitness, while the second objective was to systematically assess the overall quality of evidence. Overall, $81.6 \%$ of the studies showed positive associations between AST and PA levels with moderate quality of evidence. For body composition outcomes, there was substantial inconsistency in study findings; thus, the quality of evidence remains very low. Studies that examined differences in cardiovascular fitness between walkers and passive travelers or that did not analyze walking and cycling separately reported conflicting evidence; hence the quality of evidence was considered 
"Associations Between Active School Transport and Physical Activity, Body Composition and Cardiovascular Fitness: A Systematic Review of 68 Studies" by Larouche R et al.

Journal of Physical Activity \& Health

2012 Human Kinetics, Inc.

very low. However, cyclists had significantly better cardiovascular fitness in all five studies in which cycling was analyzed separately, and this evidence is of moderate quality.

Following the publication of the two earlier reviews that assessed differences in daily PA levels between active and passive travelers ${ }^{20,21}$, the four first intervention studies on this topic were published. Three of these studies reported that walking school bus interventions led to an increase in time spent in daily MVPA (2.2 to 17.5 additional minutes depending on the frequency of AST). ${ }^{44,45,52}$ The other intervention study reported a reduced seasonal decline in daily PA among children who received a classroom-based AST intervention. ${ }^{102}$ These studies provide some evidence suggesting that differences in PA between active and passive travelers cannot solely be explained by the former being more inclined towards PA. This is coherent with previous studies that revealed no association between travel mode and weekend PA patterns. ${ }^{39,41 \text {, }}$ ${ }^{50,55}$ Overall, differences in accelerometry-measured PA between active and passive travelers varied between 0 and 45 minutes of daily MVPA. Two studies have shown that differences in PA between active and passive travelers were greater in children living further away from school $^{30,50}$, suggesting a possible dose-response relationship. Similarly, most studies using pedometers and questionnaires have shown that active travelers were more active across the full day.

There was conflicting evidence regarding the association between AST and body composition outcomes, and these findings may be explained by several factors. It is possible to suppose that individuals may compensate for the energy expenditure resulting from AST in the rest of the day, either by: 1) engaging in less PA; 2) engaging in more sedentary behavior; and 3) increasing their energy intake. While the current findings suggest that it is unlikely that active travelers do less PA during the rest of the day, most of the reviewed studies have not assessed the 
"Associations Between Active School Transport and Physical Activity, Body Composition and Cardiovascular Fitness: A Systematic Review of 68 Studies" by Larouche R et al.

Joumal of Physical Activity \& Health

O 2012 Human Kinetics, Inc.

impact of AST on sedentary behavior and energy intake. It has also been argued that the absence of significant differences in several studies could be due to a lack of statistical power, or the lack of analyses differentiating between walking and cycling ${ }^{103}$, or by not controlling for distance in the analyses ${ }^{64}$. In addition, socioeconomic status could be a confounding variable. There is indeed evidence suggesting that low socioeconomic status is associated with higher BMI indices $^{104,105}$, and that children from lower income households are more likely to engage in $\mathrm{AST}^{106,107}$. Finally, it is noteworthy that conflicting associations between PA and body composition have also been observed in intervention studies ${ }^{108,109}$, emphasizing the complexity of body weight regulation ${ }^{110,111}$.

All five studies that have examined cycling separately have shown that cyclists had significantly greater cardiovascular fitness. However, the results are inconsistent for walking or for AST in general. This difference is possibly due to higher physical activity intensity among cyclists than walkers, as suggested by Shephard ${ }^{15}$. Similarly, a randomized controlled trial in which Finnish adults were assigned to 10 weeks of daily active or passive travel to work led to a $7 \%$ increase in $\mathrm{VO}_{2} \mathrm{max}$ in cyclists, compared to only $2 \%$ in walkers. ${ }^{112}$ In addition, longitudinal findings from the European Youth Heart Study illustrated a consistent pattern of reduced cardiovascular disease risk factors in adolescents who cycled to school, but not in those who walked. ${ }^{90}$ Hence, the current findings emphasize that future AST studies should analyze walking and cycling separately.

\section{Limitations of the studies reviewed.}

Although study quality was rated as moderate or strong for $69.1 \%$ of the studies based on the Effective Public Health Practice Project tool, few studies have given details regarding the validity and reliability of the questions used to assess mode of transportation. However, current 
"Associations Between Active School Transport and Physical Activity, Body Composition and Cardiovascular Fitness: A Systematic Review of 68 Studies" by Larouche R et al.

Journal of Physical Activity \& Health

c 2012 Human Kinetics, Inc.

questionnaires have high convergent validity $($ kappa $=0.77-0.87)$ and test-retest reliability

$(\mathrm{kappa}=0.82-0.97) .{ }^{113-116}$ It was considered that the questions that were very similar to those

that have been shown to be valid had reasonable face validity; thus, studies that did not report the validity and reliability of their travel assessment were not "penalized" on the "methods" component of the tool.

Only three intervention studies have assessed at least one of the outcome variables considered in the present review and the two pilot randomized controlled trials only examined differences in physical activity level over a short period of time. Therefore, to further improve the quality of evidence, there is a clear need for more and longer prospective intervention studies. Future studies could evaluate the impact of existing programs that promote AST (i.e. walking school buses, Safe Routes to School and classroom based approaches) on PA levels and health-related outcomes. The long-term sustainability of such initiatives needs to be further assessed. For instance, Kingham and Ussher described several challenges to the durability of walking school buses in New Zealand. ${ }^{117}$ The findings of McMinn et al ${ }^{102}$ suggest that future intervention studies should also take into consideration the impact of seasonal variations in AST on PA levels.

In addition, as Faulkner and colleagues ${ }^{20}$ pointed out, some studies classified children using AST only one day per week as active travelers. This can potentially bias the results toward the null hypothesis (e.g. the classification of children using AST once per week as active travelers is likely to reduce the magnitude of the effect). Another classification issue is that several studies have assessed mode of transport to school, but not from school; and some studies included in the current review have found that children who used AST to and from school were more active than those who did only one active trip. To avoid such biases, future studies should 
"Associations Between Active School Transport and Physical Activity, Body Composition and Cardiovascular Fitness: A Systematic Review of 68 Studies" by Larouche R et al.

Joumal of Physical Activity \& Health

○ 2012 Human Kinetics, Inc.

consider assessing AST as a continuous variable (i.e. frequency * distance). This approach would provide an estimate of the weekly distance accumulated through AST which should facilitate the assessment of dose-response relationships.

Another limitation of current knowledge is that most studies did not evaluate the doseresponse relationship between AST and health-related outcomes in children and adolescents. Only two studies have examined the impact of distance on the relationship between AST and body composition. ${ }^{64,70}$ Similarly, only two studies reported the effect sizes of differences in PA between active and passive travelers. ${ }^{54,102}$

Finally, it should be noted that self-report instruments may overestimate PA levels ${ }^{23}$ while pedometers and accelerometers underestimate PA during cycling. ${ }^{24}$ The latter may partially explain the lack of significant differences between cyclists and passive travelers observed in some studies. ${ }^{38,41,48}$ Future studies should develop and evaluate strategies to overcome this methodological limitation.

\section{Limitations and strengths of the review.}

Meta-analyses were precluded due to the wide heterogeneity in study methodologies and analyses. Further, the funnel plot and related statistical methods for detecting publication bias could not be used because a consistent measure of effect across studies was not available. However, to minimize publication bias, 10 key informants from several different countries were consulted and the search strategy included unpublished theses indexed in the ProQuest database.

The current review has several strengths. First, the search strategy was designed with a professional research librarian, and studies in both French and English were considered eligible. To our knowledge, this is the first review that assessed the impact of AST on objective and subjective measures of physical activity, and the findings indicate that the direction of the 
"Associations Between Active School Transport and Physical Activity, Body Composition and Cardiovascular Fitness: A Systematic Review of 68 Studies" by Larouche R et al.

Joumal of Physical Activity \& Health

Q 2012 Human Kinetics, Inc.

findings is consistent regardless of the type of PA measurement. Moreover, earlier reviews included almost exclusively cross-sectional studies whereas the current review included two pilot randomized control trials, two quasi-experimental studies and ten prospective studies. Finally, both the quality of overall evidence and the quality of individual studies were rigorously and transparently evaluated using GRADE and the Effective Public Health Practice Project tool.

\section{Conclusion}

The vast majority of the studies have shown a positive relationship between AST and physical activity levels. In addition, there is consistent evidence that cycling to/from school is associated with greater cardiovascular fitness and that the mode of transport explains a substantial proportion of the variance in cardiovascular fitness. However, the association between AST and indicators of body composition remains equivocal. Potential reasons for this conflicting evidence include compensatory behaviors (e.g., reducing PA in other parts of the day), inconsistencies in measurement of active travel and/or physical activity, confounding variables (i.e. low socioeconomic status may be positively associated with both AST and BMI), not controlling for distance in the analyses or not analyzing cyclists and walkers separately. Findings of studies that examined differences in fitness between walkers and passive travelers are also inconsistent. This inconsistency may be due to the lower exercise intensity of walking (in comparison to cycling), which may be insufficient to increase fitness in healthy children and youth. ${ }^{15}$

Interestingly, a recent systematic review concluded that most AST interventions have led to increases in the proportion of children walking and cycling to school. ${ }^{118}$ Given the positive association between AST and physical activity levels, and between cycling to/from school and cardiovascular fitness, further investments for the implementation and evaluation of AST 
"Associations Between Active School Transport and Physical Activity, Body Composition and Cardiovascular Fitness: A Systematic Review of 68 Studies" by Larouche R et al.

Journal of Physical Activity \& Health

2012 Human Kinetics, Inc.

interventions are warranted. It is also noteworthy that reducing the proportion of motorized school trips could yield important environmental co-benefits (i.e. reduction in greenhouse gases emissions, particulate matter, carbon monoxide, nitric oxides, volatile organic compounds, etc.). ${ }^{119}$ In turn, the particulate matter emitted by vehicles increases the likelihood of cardiovascular and respiratory diseases. ${ }^{120-121}$ In this perspective, active transport represents a powerful strategy to simultaneously address several environmental and public health problems. ${ }^{14}$, 122,123

These findings clearly emphasize the need for more intervention studies to increase the quality of evidence. Given the marked difference in the impact of walking and cycling on cardiovascular fitness, analyzing these modes of transportation separately is warranted. Finally, to facilitate the examination of dose-response relationships, and to reduce classification biases, future studies should assess the relationship between AST and health-related outcomes using a continuous measure of AST.

\section{Acknowledgements}

RL holds a Frederick Banting and Charles Best Canada Graduate Scholarship from the Canadian Institutes of Health Research (CIHR). TJS holds Doctoral Research Awards from the Canadian Institutes of Health Research (CIHR) and from the Canadian Diabetes Association. The authors wish to thank Dr. Margaret Sampson for her help in preparing the search strategy, Dr. Nick Barrowman for his advice on the analyses, and the 10 researchers who kindly provided references for the review. 
"Associations Between Active School Transport and Physical Activity, Body Composition and Cardiovascular Fitness: A Systematic Review of 68 Studies" by Larouche R et al.

Joumal of Physical Activity \& Health

2012 Human Kinetics, Inc.

\section{References}

1. US Department of Health and Human Services. Physical Activity Guidelines Advisory Committee Report, 2008. Washington, DC. 2008.

2. Tremblay MS, Warburton DER, Janssen I, Paterson DH, Latimer AE, Rhodes RE, Kho ME, Hicks A, Leblanc AG, Zerh L, Murumets K, Duggan M. (2011). New Canadian physical activity guidelines. Appl Physiol Nutr Metabol. 2011;36(1):36-46.

3. Colley RC, Garriguet D, Janssen I, Craig CL, Clarke J, Tremblay MS. Physical activity of Canadian children and youth: Accelerometer results from the Canadian Health Measures Survey. Health Reports. 2011;22(1).

4. Troiano RP, Berrigan D, Dodd KW, Mâsse LC, Tilert T, McDowell, M. Physical activity in the United States measured by accelerometer. Med Sci Sports Exerc.2008;40(1):181188.

5. Bauman A, Finegood DT, Matsudo V. International perspectives on the physical inactivity crisis - Structural solutions over evidence generation? Prev Med. 2009;49:309312.

6. Andersen LB, Harro M, Sardinha LB, Froberg K, Ekelund U, Brage S, Anderssen SA. Physical activity and clustered cardiovascular risk in children: a cross-sectional study (The European Youth Heart Study). Lancet. 2006;368:299-304.

7. Janssen I, Katzmarzyk PT, Boyce WF, King MA, Pickett W. Overweight and obesity in Canadian adolescents and their association with dietary habits and physical activity patterns. J Adolesc Health. 2004;35(5):360-367. 
"Associations Between Active School Transport and Physical Activity, Body Composition and Cardiovascular Fitness: A Systematic Review of 68 Studies" by Larouche R et al.

Joumal of Physical Activity \& Health

2012 Human Kinetics, Inc.

8. Jiménez-Pavón D, Kelly J, Reilly JJ. Associations between objectively measured habitual physical activity and adiposity in children and adolescents: Systematic review. Int $J$ Pediatr Obes. 2010;5:3-18.

9. Olds TS, Dollman J. Are secular declines in children's aerobic fitness explained by changes in fatness? Pediatr Exerc Sci. 2003;16(3):201-209.

10. Tremblay MS, Shields M, Laviolette M, Craig CL, Janssen I, Connor Gorber S. Fitness of Canadian children and youth: Results from the 2007-2009 Canadian Health Measures Survey. Health Reports. 2010;21(1):1-14.

11. Tudor-Locke C, Ainsworth BE, Popkin BM. Active commuting to school: An overlooked source of children's physical activity? Sports Med. 2001;31(5):309-313.

12. Chapman L. Transport and climate change: A review. J Transport Geography. 2007;15(5):354-367.

13. Frank LD, Greenwald MJ, Winkelman S, Chapman J, Kavage S. Carbonless footprints: promoting health and climate stabilization through active transportation. Prev Med. 2010;50(Suppl. 1):S99-S105

14. Woodcock J, Edwards P, Tonne C, Armstrong BG, Ashiru O et al. Public health benefits of strategies to reduce greenhouse-gas emissions: urban land transport. Lancet. 2009;374(9705):1930-1943.

15. Shephard RJ. Is active commuting the answer to population health? Sports Med. 2008;38(9):751-758.

16. Buliung RN, Mitra R, Faulkner G. Active school transportation in the Greater Toronto Area, Canada: An exploration of trends in space and time (1986-2006). Prev Med. $2009 ; 48: 507-512$. 
"Associations Between Active School Transport and Physical Activity, Body Composition and Cardiovascular Fitness: A Systematic Review of 68 Studies" by Larouche R et al.

Joumal of Physical Activity \& Health

Q 2012 Human Kinetics, Inc.

17. McDonald NC. Active transportation to school: Trends among U.S. schoolchildren, 1969-2001. Am J Prev Med. 2007;32(6):509-516.

18. van der Ploeg, HP, Merom D, Corpuz G, Bauman AE. Trends in Australian children travelling to school 1971-2003: Burning petrol or carbohydrates? Prev Med. 2008;46:6062.

19. Grize L, Bringolf-Isler B, Martin E, Braun-Fahrländer C. Trends in active transport to school among Swiss school children and its associated factors: three cross-sectional surveys 1994, 2000 and 2005. Int J Behav Nutr Phys Act. 2010;7(28). http://www.ijbnpa.org/content/7/1/28.

20. Faulkner GEJ, Buliung RN, Flora PK, Fusco C. Active school transport, physical activity levels and body weight of children and youth: a systematic review. Prev Med. 2009;49:38.

21. Lee MC, Orenstein MR, Richardson MC. Systematic review of active commuting to school and children's physical activity and weight. J Phys Act Health. 2008;5(6):930949.

22. Lubans DR, Boreham CA, Kelly P, Foster CE. The relationship between active travel to school and health-related fitness in children and adolescents: a systematic review. Int $J$ Behav Nutr Phys Act. 201 1;8(5). http://www.ijbnpa.org/content/8/1/5/abstract

23. Adamo KB, Prince SA, Tricco AC, Connor-Gorber S, Tremblay MS. A comparison of indirect versus direct measures for assessing physical activity in the pediatric population: a systematic review. Int J Pediatr Obes. 2009;4(1):2-27.

24. Corder K, Brage S, Ekelund U. Accelerometers and pedometers: methodology and clinical application. Curr Opin Clin Nutr Metab Care. 2007;10:597-603. 
"Associations Between Active School Transport and Physical Activity, Body Composition and Cardiovascular Fitness: A Systematic Review of 68 Studies" by Larouche R et al.

Journal of Physical Activity \& Health

(O) 2012 Human Kinetics, Inc.

25. Effective Public Health Practice Project. Quality Assessment Tool for Quantitative Studies. Downloaded from http://www.ephpp.ca/Tools.html Accessed July 12, 2011.

26. Oja P, Titze S, Bauman A, de Geus B, Krenn P, Reger-Nash B, Kohlberger T. Health benefits of cycling: a systematic review. Scand J Med Sci Sports. 2011;21:496-509.

27. de Bourdeaudhuij I, Van Cauwenberghe E, Spittaels H, Oppert J-M, Rostami C, Brug J, Van Lenthe F, Lobstein T, Maes L. School-based interventions promoting both physical activity and healthy eating in Europe: a systematic review within the HOPE project. Obes Rev. 2011;12:205-216.

28. Guyatt, GH, Oxman AD, Vist GE, Kunz R, Falck-Ytter Y, Alonso-Coello P, Schünemann HJ. GRADE: An emerging consensus on rating quality of evidence and strength of recommendations. Br Med J. 2008;336:924-926.

29. Guyatt, GH, Oxman AD, Vist GE, Kunz R, Falck-Ytter Y, Schünemann HJ. GRADE: What is "quality of evidence" and why is it important to clinicians? $\mathrm{Br}$ Med J. 2008;336:995-998.

30. van Sluijs EMF, Fearne VA, Mattocks C, Riddoch C, Griffin SJ, Ness A. The contribution of active travel to children's physical activity levels: Cross-sectional results from the ALSPAC study. Prev Med. 2009;48:519-524.

31. Nilsson A, Andersen LB, Ommundsen Y, Froberg K, Sardinha LB, Piehl-Aulin K, Ekelund U. Correlates of objectively assessed physical activity and sedentary time in children: a cross-sectional study (the European Youth Heart Study). BMC Public Health. 2009;9(322). http://www.biomedcentral.com/1471-2458/9/322 
"Associations Between Active School Transport and Physical Activity, Body Composition and Cardiovascular Fitness: A Systematic Review of 68 Studies" by Larouche R et al.

Journal of Physical Activity \& Health

02012 Human Kinetics, Inc.

32. Cooper AR, Page AS, Wheeler BW, Griew P, Davis L, Hillsdon M, Jago R. Mapping the walk to school using accelerometry combined with a global positioning system. Am J Prev Med. 2010;38(2):178-183.

33. Lofgren B, Stenevi-Lundgren S, Dencker M, Karlsson MK. The mode of school transportation in pre-pubertal children does not influence the accrual of bone mineral or the gain in bone size - two year prospective data from the paediatric osteoporosis preventive (POP) study. BMC Musculoskelet Disord. 2010;11(25). http://www.biomedcentral.com/1471-2474/11/25

34. Cooper AR, Wedderkopp N, Jago R, Kristensen PL, Moller NC, Froberg K, Page AS, Andersen LB. Longitudinal associations of cycling to school with adolescent fitness. Prev Med. 2008;47(3):324-328.

35. Ford B, Bailey R, Coleman D, Woolf-May K, Swaine I. Activity levels, dietary energy intake, and body composition in children who walk to school. Pediatr Exerc Sci. 2007;19:393-407.

36. Alwis G, Linden C, Dencker M, Stenevi-Lundgren S, Gardsell P, Karlsson MK. Bone mineral accrual and gain in skeletal width in pre-pubertal school children is independent of the mode of school transportation - one-year data from the prospective observational pediatric osteoporosis prevention (POP) study. BMC Musculoskelet Disord. 2007;8(66). http://www.biomedcentral.com/1471-2474/8/66

37. Saskvig BI, Catellier DJ, Pfeiffer K, Schmitz KH, Conway T, Going S., Ward D, Strikmiller P, Treuth MS. Travel by walking before and after school and physical activity among adolescent girls. Arch Pediatr Adolesc Med. 2007;161:153-158. 
"Associations Between Active School Transport and Physical Activity, Body Composition and Cardiovascular Fitness: A

Systematic Review of 68 Studies" by Larouche R et al.

Joumal of Physical Activity \& Health

2012 Human Kinetics, Inc.

38. Cooper AR, Wedderkopp N, Wang N, Andersen LB, Froberg K, Page AS. Active travel to school and cardiovascular fitness in Danish children and adolescents. Med Sci Sports Exerc. 2006;38(10):1724-1731.

39. Sirard JR, Riner WF Jr., McKiver KL, Pate RR. Physical activity and active commuting to elementary school. Med Sci Sports Exerc. 2005;37(12):2062-2069.

40. Alexander LM, Inchley J, Todd J, Currie D, Cooper AR, Currie C. The broader impact of walking to school among adolescents: seven day accelerometry based study. $\mathrm{Br} \mathrm{Med} J$. 2005;331:1061-1062.

41. Cooper AR, Andersen LB, Wedderkopp N, Page AS, Froberg K. Physical activity levels of children who walk, cycle, or are driven to school. Am J Prev Med. 2005;29(3):179184.

42. Cooper AR, Page AS, Foster LJ, Qahwaji D. Commuting to school: are children who walk more active. Am J Prev Med. 2003;25(4):273-276.

43. Tudor-Locke C, Ainsworth BE, Adair LS, Popkin BM. Objective physical activity monitoring of Filipino youth stratified for commuting mode to school. Med Sci Sports Exerc. 2003;35(3):465-471.

44. Heelan KA, Abbey BM, Donnelly JE, Mayo MS, Welk GJ. Evaluation of a walking school bus for promoting physical activity in youth. JPhys Act Health. 2009;6:560-567.

45. Sirard JR, Alhassan S, Spencer TR, Robinson TN. Changes in physical activity from walking to school. J Nutr Educ Behav. 2008;40(5):324-326.

46. Rosenberg DE, Sallis JF, Conway TL, Cain KL, McKenzie TL. Active transport to school over 2 years in relation to weight status and physical activity. Obesity, 2006;14(10):1771-1776. 
"Associations Between Active School Transport and Physical Activity, Body Composition and Cardiovascular Fitness: A Systematic Review of 68 Studies" by Larouche R et al.

Joumal of Physical Activity \& Health

O 2012 Human Kinetics, Inc.

47. Mendoza JA, Watson K. Nguyen N, Cerin E, Baranwski T, Nicklas TA. Active commuting to school and association with physical activity and adiposity among US youth. JPhys Act Health. 2011;8(4):488-495.

48. Chillón P, Ortega FB, Ruiz JR, Veidebaum T, Oja L, Mäestu J, Sjöström M. Active commuting to school in children and adolescents: An opportunity to increase physical activity and fitness. Scand J Public Health. 2010;38(8):873-879.

49. Mendoza JA, Watson K, Baranowski T, Nicklas TA, Uscanga DK. Ethnic minority children's commuting to school and associations with physical activity and pedestrian safety behaviors. Journal of Applied Research in Children, 2010;1(1). http://digitalcommons.library.tmc.edu/childrenatrisk/voll/iss1/4/

50. Panter JR, Jones AP, van Sluijs EMF, Griffin SJ. The influence of distance to school on the associations between active commuting and physical activity. Pediatr Exerc Sci. $2011 ; 23(1): 72-86$.

51. Chillon P, Ortega FB, Ruiz JR, de Bourdeaudhuij I, Martinez-Gomez D, VicenteRodriguez G, Widhalm K, Molnar D, Gottrand F, Gonzalez-Gross M, Ward DS, Moreno LA, Castillo MJ, Sjostrom M, HELENA study group. Active commuting and physical activity in adolescents from Europe: Results from the HELENA study. Pediatr Exerc Sci. $2011 ; 23(2): 207-217$.

52. Mendoza JA, Watson K, Baranowski T, Nicklas TA, Uscanga DK, Hanfling MJ. The walking school bus and children's physical activity: a pilot cluster randomized controlled trial. Pediatrics. 2011;138(3):e537-e544. 
"Associations Between Active School Transport and Physical Activity, Body Composition and Cardiovascular Fitness: A Systematic Review of 68 Studies" by Larouche R et al.

Journal of Physical Activity \& Health

2012 Human Kinetics, Inc.

53. Abbott RA, MacDonald D, Nambiar S, Davies PSW. The association between walking to school, daily step counts and meeting step targets in 5- to 17-years-old Australian children. Pediatr Exerc Sci. 2009;21:520-532.

54. Loucaides CA, Jago R. Differences in physical activity by gender, weight status and travel mode to school in Cypriot children. Prev Med. 2008;47:107-111.

55. Duncan EK, Duncan JS, Schofield G. Pedometer-determined physical activity and active transport in girls. Int J Behav Nutr Phys Act. 2008;5(2).

http://www.ijbnpa.org/content/5/1/2

56. Hohepa M, Schofield G, Kolt GS, Scragg R, Garrett N. Pedometer-determined physical activity levels of adolescents: differences by age, sex, time of week, and transportation mode to school. J Phys Act Health, 2008;5(Suppl.1):S140-S152.

57. Cuddihy T, Davidson M, Michaud-Tomson L. Walk to school - Does it make a difference in children's physical activity levels. ACHPER Healthy Lifestyles Journal. $2003 ; 50(3-4): 16-24$

58. Larouche R, Lloyd M, Knight E, Tremblay MS. Relationship between active school transport and body mass index in Grades 4-6 children. Pediatr Exerc Sci. $2011 ; 23(3): 322-330$.

59. Johnson TG, Brusseau TA, Darst PW, Kulinna PH, White-Taylor J. Step counts of nonwhite minority children and youth, by gender, grade level, race/ethnicity, and mode of school transportation. J Phys Act Health. 2010;7:730-736.

60. Murtagh EM, Murphy MH. Active travel to school and physical activity levels of Irish primary schoolchildren. Pediatr Exerc Sci. 2011;23(2):230-236. 
"Associations Between Active School Transport and Physical Activity, Body Composition and Cardiovascular Fitness: A Systematic Review of 68 Studies" by Larouche R et al.

Joumal of Physical Activity \& Health

○2012 Human Kinetics, Inc.

61. McCormack GR, Giles-Corti B, Timperio A, Wood G, Villanueva K. Int J Behav Nutr Phys Act. 2011;8(30). http://www.ijbnpa.org/content/8/1/30

62. Trang NHHD, Hong TK, Dibley MJ, Sibbritt BW. Factors associated with physical inactivity in adolescents in Ho Chi Minh City, Vietnam. Med Sci Sports Exerc. $2009 ; 41(7): 1374-1383$.

63. Voss C, Sandercock G. Aerobic fitness and mode of travel to school in English schoolchildren. Med Sci Sports Exerc. 2010;42(2):281-287.

64. Landsberg B, Plachta-Danielzik S, Much D, Johannsen N, Lange D, Müller MJ. Associations between active commuting to school, fat mass and lifestyle factors in adolescents: the Kiel Obesity Prevention Study. Eur J Clin Nutr. 2008;62:739-747.

65. Spinks A, Macpherson A, Bain C, McClure R. Determinants of sufficient daily activity in Australian primary school children. J Paediatr Child Health. 2006;42:674-679.

66. Tudor-Locke C, Neff LG, Ainsworth BE, Addy CL, Popkin BM. Omission of active commuting to school and the prevalence of children's health-related physical activity level: the Russian Longitudinal Monitoring Study. Child Care Health Dev. $2002 ; 28(6): 507-512$.

67. Wen LM, Merom D, Rissel C, Simpson JM. Weight status, mode of travel to school and screen time: a cross-sectional survey of children 10-13 years in Sydney. Health Promot J Austr. 2010;21:57-63.

68. Andersen LB, Lawlor DA, Cooper AR, Froberg K, Anderssen SA. Physical fitness in relation to transport to school in adolescents: the Danish youth and sports study. Scand J Med Sci Sports. 2009;19:406-411. 
"Associations Between Active School Transport and Physical Activity, Body Composition and Cardiovascular Fitness: A Systematic Review of 68 Studies" by Larouche R et al.

Journal of Physical Activity \& Health

02012 Human Kinetics, Inc.

69. Loucaides CA, Plotnikoff RC, Bercovitz K. Differences in the correlates of physical activity between urban and rural Canadian youth. J School Health. 2007;77:164-170.

70. Heelan KA, Donnelly JE, Jacobsen DJ, Mayo MS, Washburn R, Greene L. Active commuting to and from school BMI in elementary school children - preliminary data. Child Care Health Dev. 2005;31(3):341-349.

71. Van Dyck D, de Bourdeaudhuij I, Cardon G, Deforche B. Criterion distances and correlates of active transportation to school in Belgian older adolescents. Int J Behav Nutr Phys Act. 2010;7(87). http://www.ijbnpa.org/content/7/1/87

72. Silva KS, Vasques DG, Martins CO, Williams LA, Lopes AS. Active commuting: Prevalence, barriers, and associated variables. J Phys Act Health. 2011;8:750-757.

73. Baig F, Hameed MA, Li M, Shorthouse G, Roalfe AK, Daley A. Association between active commuting to school, weight and physical activity status in ethnically diverse adolescents predominately living in deprived communities. Public Health. 2009;123:3941.

74. Madsen KA, Gosliner W, Woodward-Lopez G, Crawford PB. Physical activity opportunities associated with fitness and weight status in adolescents in low-income communities. Arch Pediatr Adolesc Med. 2009;163(11):1014-1021.

75. Silva KS, Lopes AS. Excess weight, arterial pressure, and physical activity in commuting to school: Correlations. Arq Bras Cardiol. 2008;91(2):84-91.

76. Collins AE, Pakiz B, Rock CL. Factors associated with obesity in Indonesian adolescents. Int J Pediatr Obes. 2008;3:58-64.

77. Li Y, Zhai F, Yang X, Schouton EG, Hu X, He Y, Luan D, Ma G. Determinants of childhood overweight and obesity in China. Br J Nutr. 2007;97: 210-215. 
"Associations Between Active School Transport and Physical Activity, Body Composition and Cardiovascular Fitness: A Systematic Review of 68 Studies" by Larouche R et al.

Joumal of Physical Activity \& Health

2012 Human Kinetics, Inc.

78. Klein-Platat C, Oujaa M, Wagner A, Hann MC, Arveiler DC, Schlienger JL, Simon C. Physical activity is inversely related to waist circumference in 12-y-old French adolescents. Int J Obes. 2005;29:9-14.

79. Jansen W, Mackenbach JP, Joosten-Van Zwanenburg E, Brug J. Weight status, energybalance behaviours and intentions in 9-12-years-old inner-city children. J Hum Nutr Diet. 2010;23:85-96.

80. Bere E, Seiler S, Eikemo TA, Oenema A, Brug J. The association between cycling to school and being overweight in Rotterdam (The Netherlands) and Kristiansand (Norway). Scand J Med Sci Sports. 2011;21:48-53.

81. Pabayo R, Gauvin L, Barnett TA, Nikiéma B, Séguin L. Sustained active transportation is associated with a favorable body mass index trajectory across the early school years: Findings from the Québec Longitudinal Study of Child Development birth cohort. Prev Med. 2010;50(Suppl. 1):S59-S64.

82. Aires L, Mendonca, D, Silva G, Gaya AR, Santos MP, Ribeiro C, Mota J. A 3-year longitudinal analysis of changes in body mass index. Int J Sports Med. 2010;31(2):133137.

83. Wijga AH, Scholtens S, Bemelmans WJE, Kerkhof M, Koppelman GH, Brunekreef B, Smit, HA. Diet, screen time, physical activity, and childhood overweight in the general population and in high risk subgroups: prospective analyses in the PIAMA birth cohort. $J$ Obes. 2010. http://downloads.hindawi.com/journals/jobes/2010/423296.pdf

84. Kong AS, Burks N, Conklin C, Roldan C, Skipper B, Scott S, Sussman AL, Leggott J. A pilot walking school bus to prevent obesity in Hispanic elementary school children: Role 
"Associations Between Active School Transport and Physical Activity, Body Composition and Cardiovascular Fitness: A Systematic Review of 68 Studies" by Larouche R et al.

Journal of Physical Activity \& Health

C 2012 Human Kinetics, Inc.

of physician involvement with the school community. Clinical Pediatr. 2010;49(10):989991.

85. Ostergaard L, Grontved A, Borrestad LAB, Froberg K, Gravesen M, Andersen LB. Cycling to school is associated with lower BMI and lower odds of being overweight or obese in a large population-based study of Danish adolescents. J Phys Act Health. In press.

86. Bere E, Oenema A, Prins RG, Seiler S, Brug J. Longitudinal associations between cycling to school and weight status. Int J Pediatr Obes. 2011;6:182-187.

87. Heelan KA. Active commuting to and from school and BMI in elementary school children. [PhD thesis]. University of Kansas; 2002.

88. Tudor-Locke C, Ainsworth BE, Adair LS, Popkin BM. Physical activity in Filipino youth: the Cebu Longitudinal Health and Nutrition Survey. Int J Obes. 2003;27:181-190.

89. Sirard JR. Active commuting to school and physical activity in elementary school children. [PhD thesis]. University of South Carolina; 2003.

90. Andersen LB, Wedderkopp N, Kristensen P, Moller NC, Froberg K, Cooper AR. Cycling to school and cardiovascular risk factors: a longitudinal study. J Phys Act Health. 2011;8:1025-1033.

91. Owen CG, Nightingale CM, Rudnicka AR, van Sluijs EMF, Ekelund U, Cook DG, Whincup PH. Travel to school and physical activity levels in 9-10 years-old UK children of different ethnic origin; Child Heart and Health Study in England (CHASE). PLoS ONE. 2012:7(2):e30932. 
"Associations Between Active School Transport and Physical Activity, Body Composition and Cardiovascular Fitness: A Systematic Review of 68 Studies" by Larouche R et al.

Journal of Physical Activity \& Health

2012 Human Kinetics, Inc.

92. Roth MA, Millett CJ, Mindell JS. The contribution of active travel (walking and cycling) in children to overall physical activity level: a national cross sectional study. Prev Med. 2012;54(2):134-139.

93. Daly-Smith AJW, McKenna J, Radley D, Long J. The impact of additional weekdays of active commuting to school on children achieving a criterion of $300+$ minutes of moderate-to-vigorous physical activity. Health Educ J. 2011:70(4):428-434.

94. Meron D, Rissel C, Reinten-Reynolds T, Hardy LL. Changes in active travel of school children from 2004 to 2010 in New South Wales, Australia. Prev Med. 201 1;53(6):408410.

95. Goodman A, Mackett, RL, Paskins J. Activity compensation and activity synergy in British 8-12 year old. Prev Med. 2011;53:293-298.

96. Andegiorgish AK, Wang J, Zhang X, Liu X, Zhu H. Prevalence of overweight, obesity, and associated risk factors among school children and adolescents in Tianjin, China. Eur J Pediatr. 2012;171:697-703.

97. Aires L, Pratt M, Lobelo F, Santos RM, Santos MP, Mota J. Associations of cardiorespiratory fitness in children and adolescents with physical activity, active commuting to school, and screen time. J Phys Act Health. 2011;8(Suppl. 2):S198-S205.

98. Arango CM, Parra, DC, Eyler A, Sarmineto O, Mantilla SC, Gomez LF, Lobelo P. Walking or bicycling to school and weight status among adolescents from Montería, Colombia. J Phys Act Health. 2011;8(Suppl. 2):S171-S177.

99. King AC, Parkinson KN, Adamson AJ, Murray L, Besson H, Reilly JJ, Basterfield L et al. Correlates of objectively measured physical activity and sedentary behaviour in English children. Eur J Public Health. 2011;21(4):424-431. 
"Associations Between Active School Transport and Physical Activity, Body Composition and Cardiovascular Fitness: A Systematic Review of 68 Studies" by Larouche R et al.

Joumal of Physical Activity \& Health

2012 Human Kinetics, Inc.

100. Duncan S, Duncan EK, Fernandes RA, Buonani C, Bastos KDN, Segatto AFM, Codogno JS, Gomes IC, Freitas Jr. IF. Modifiable risk factors for overweight and obesity in children and adolescents from São Paulo, Brazil. BMC Public Health. 2011;11:585. http://www.biomedcentral.com/1471-2458/11/585

101. Pabayo R. Investigating active transportation to and from school: Identification of predictors and health benefits. [PhD thesis]. Université de Montréal; 2010.

102. McMinn D, Rowe D, Murtagh S, Nelson NM. The effect of a school-based active commuting intervention on children's commuting physical activity and daily physical activity. Prev Med. In press.

103. Bere E, Andersen LB. Why no support for an association between active commuting to school and weight status in the literature. JPhys Act Health. 2009;6:533-534.

104. Janssen I, Boyce WF, Simpson K, Pickett W. Influence of individual- and area-level measures of socioeconomic status on obesity, unhealthy eating, and physical inactivity in Canadian adolescents. Am J Clin Nutr. 2006;83(1):139-145.

105. Stamatakis E, Wardle J, Cole TJ. Childhood obesity and overweight trends in England: evidence for growing socioeconomic disparities. Int J Obes. 2010;34:41-47.

106. Pabayo R, Gauvin L. Proportion of students who use various modes of transportation to and from school in a representative population-based sample of children and adolescents, 1999. Prev Med. 2008;46(1):63-66.

107. Pont K, Ziviani J, Wadley D, Bennett S, Abbott R. Environmental correlates of children's active transportation: A systematic literature review. Health Place. 2009;15:849-862. 
"Associations Between Active School Transport and Physical Activity, Body Composition and Cardiovascular Fitness: A Systematic Review of 68 Studies" by Lanouche R et al.

Joumal of Physical Activity \& Health

Q 2012 Human Kinetics, Inc.

108. Harris KC, Kuramoto LK, Schulzer M, Retallack JE. Effect of school-based physical activity interventions on body mass index in children: a meta-analysis. Can Med AssocJ. 2009;180 (7):719-726.

109. Reilly JJ, McDowell ZC. Physical activity interventions in the prevention and treatment of paediatric obesity: systematic review and critical appraisal. Proc Nutr Soc. $2003 ; 62: 611-619$.

110. Chaput J-P, Brunet M, Tremblay A. Relationship between short sleeping hours and childhood overweight/obesity: Results from the 'Quebec en Forme' project. Int J Obes. 2006;30:1080-1085.

111. Keith SW, Redden DT, Katzmarzyk PT, Boggiano MM, Hanlon EC. Putative contributors of the secular increase in obesity: exploring the roads less travelled. Int $J$ Obes. 2006;30:1585-1594.

112. Oja P, Mänttäri A, Heinonen, A, Kukkonen-Harjula, K, Laukanen R, Pasanen M, Vuori I. Physiological effects of walking and cycling to work. Scand J Med Sci Sports. $1991 ; 1: 151-157$.

113. Bere E, Bjorkelund LA. Test-retest reliability of a new self reported comprehensive questionnaire measuring frequencies of different modes of adolescents commuting to school and their parents commuting to work - the ATN questionnaire. Int J Behav Nutr Phys Act. 2009;6(68). http://www.ijbnpa.org/content/pdf/1479-5868-6-68.pdf

114. Evenson KR, Neelon B, Ball SC, Vaughn A, Ward DS. Validity and reliability of a school travel survey. J Phys Act Health. 2008;5(Suppl 1):S1-S15. 
"Associations Between Active School Transport and Physical Activity, Body Composition and Cardiovascular Fitness: A Systematic Review of 68 Studies" by Larouche R et al.

Journal of Physical Activity \& Health

- 2012 Human Kinetics, Inc.

115. McDonald NC, Dwelley AE, Combs TS, Evenson KR, Winters RH. Reliability and validity of the Safe Routes to School parent and students survey. Int J Behav Nutr Phys Act. 2011;8(56). http://www.ijbnpa.org/content/8/1/56/

116. Mendoza JA, Watson K, Baranowski T, Nicklas TA, Uscanga, DK, Hanfling, MJ. Validity of instruments to assess students' travel and pedestrian safety. BMC Public Health. 2010;10(257). http://www.biomedcentral.com/1471-2458/10/257

117. Kingham S, Ussher S. Ticket to a sustainable future: An evaluation of the long-term durability of the Walking School Bus program in Christchurch, New Zealand. Transport Policy. 2005;12(4):314-323.

118. Chillón P, Evenson KR, Vaughn A, Ward DS. A systematic review of interventions for promoting active transportation to school. Int J Behav Nutr Phys Act. 2011;8(10). http://www.ijbnpa.org/content/8/1/10

119. Marshall JD, Wilson RD, Meyer KL, Rajangam SK, McDonald NC, Wilson EJ. Vehicle emissions during children's school commuting: impact of education policy. Environ Sci Technol. 2010;44(5):1537-1543.

120. Brook RD, Rajagopalan S, Pope III A, Brook JR, Bhatnagar, A et al. Particulate matter air pollution and cardiovascular disease: an update to the scientific statement from the American Heart Association. Circulation. 2010;121:2331-2378.

121. Friedman MS, Powell KE, Hutwagner L, Graham LM, Teague WG. Impact of changes in transportation and commuting behaviors during the 1996 Summer Olympic Games in Atlanta on air quality and childhood asthma. JAMA. 2001;285(7):897-905. 
"Associations Between Active School Transport and Physical Activity, Body Composition and Cardiovascular Fitness: A

Systematic Review of 68 Studies" by Larouche R et al.

Joumal of Physical Activity \& Health

Q 2012 Human Kinetics, Inc.

122. Haines A, McMichael AJ, Smith KR, Roberts I, Woodcock J et al. Public health benefits of strategies to reduce greenhouses-gas emissions: overview and implications for policymakers. Lancet. 2009;374(9707):2104-2114.

123. Larouche $\mathrm{R}$. The environmental and population health benefits of active transport: A review. In G. Liu (Ed.) Greenhouse Gases - Emissions, Measurement and Management. InTech: Rijeka, Croatia. 2012. http://cdn.intechopen.com/pdfs/32358/InTechThe environmental and population_health_benefits_of active transport_a review.pdf 


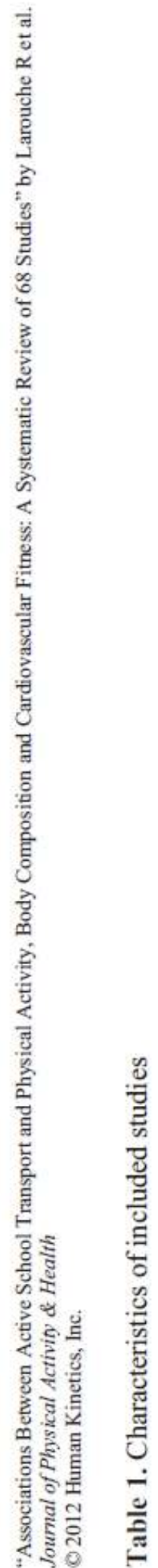

\begin{tabular}{|c|c|c|c|c|c|c|c|c|c|c|c|c|c|c|c|c|c|c|}
\hline ڤ્气 & 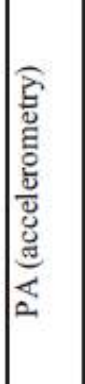 & 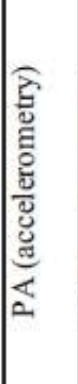 & 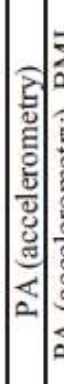 & 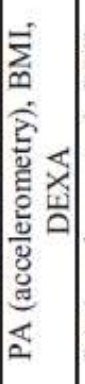 & 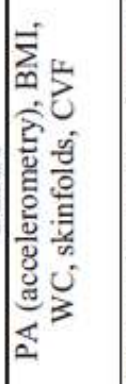 & 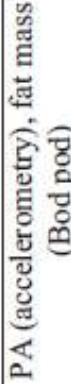 & 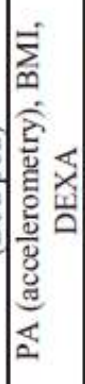 & 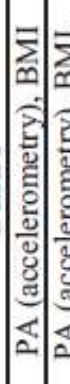 & 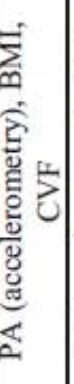 & 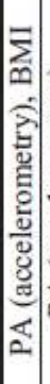 & 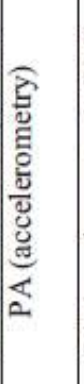 & 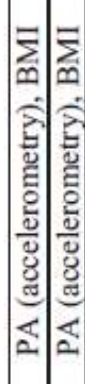 & 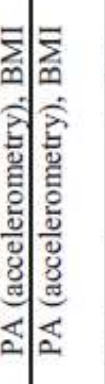 & 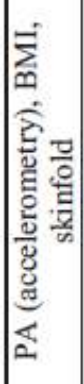 & 国 & 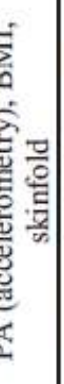 & 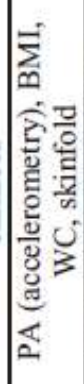 & 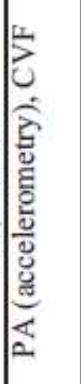 \\
\hline 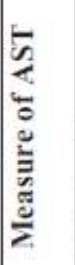 & 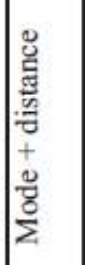 & 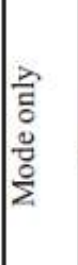 & 兽 & 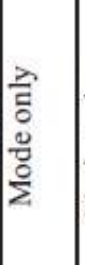 & 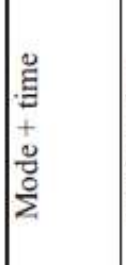 & 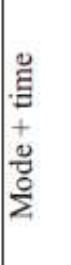 & 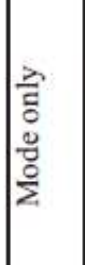 & 굴 & 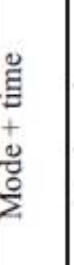 & 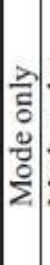 & 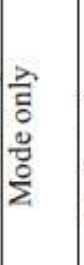 & 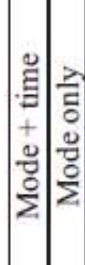 & 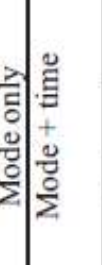 & 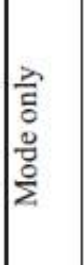 & \begin{tabular}{l|l}
0 \\
0 \\
0
\end{tabular} & & 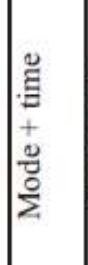 & 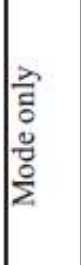 \\
\hline 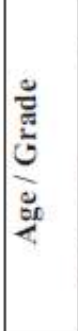 & 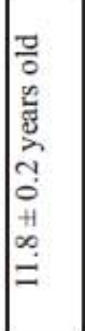 & 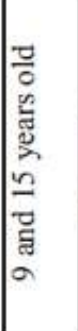 & : & 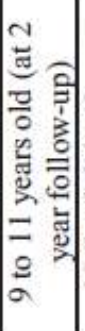 & 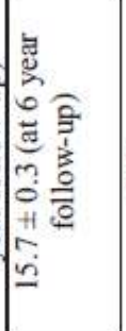 & $\overline{\bar{n}}$ & 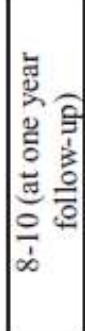 & 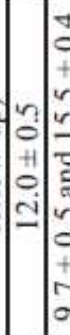 & 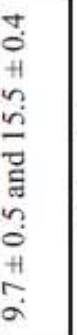 & $\mid \begin{array}{c}0 \\
0 \\
0 \\
+1 \\
\tilde{0} \\
0\end{array}$ & & & 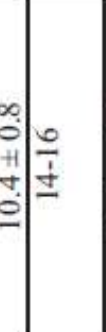 & 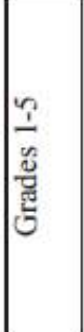 & 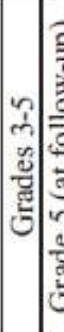 & & $\frac{a}{\dot{d}}$ & 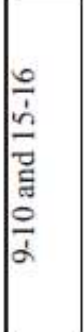 \\
\hline 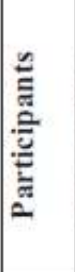 & 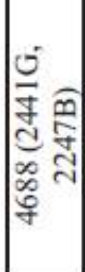 & 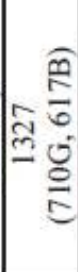 & 用 & 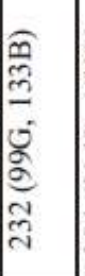 & 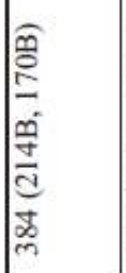 & 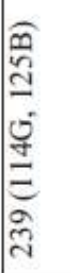 & 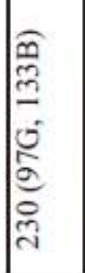 & 图部 & 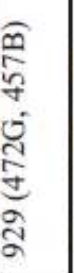 & 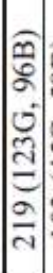 & $\begin{array}{l}\widehat{\infty} \\
\infty \\
n \\
0 \\
0 \\
0 \\
0 \\
0 \\
0 \\
0\end{array}$ & 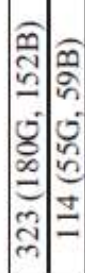 & 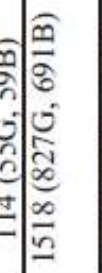 & 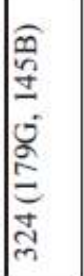 & 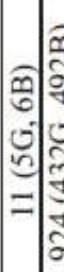 & & 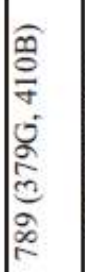 & 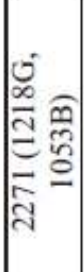 \\
\hline 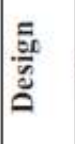 & 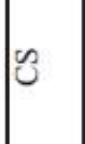 & 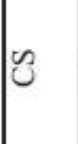 & $\mid$ & $g$ & ڤ̊ & 8 & $\stackrel{8}{2}$ & 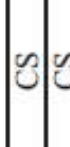 & 0 & 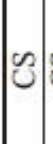 & $\theta$ & $\approx$ & 3 & 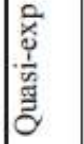 & 阁 & & 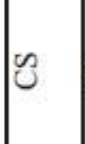 & 3 \\
\hline U⿺ & 光 & 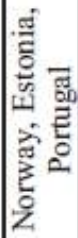 & & 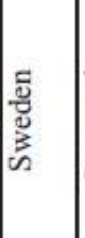 & 总 & 弚 & 总 & 结 & 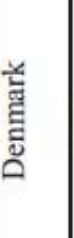 & $\mid \begin{array}{l}\ll \\
s \\
s\end{array}$ & $\angle$ & 亲咅 & 当 & S & $\begin{array}{l}< \\
0 \\
0\end{array}$ & & 氒 & 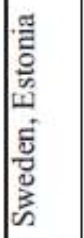 \\
\hline 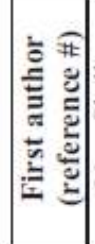 & 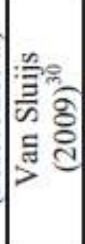 & 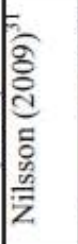 & 을. & 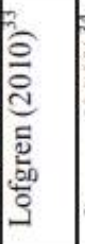 & 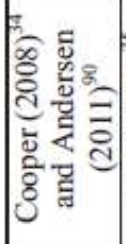 & 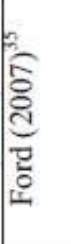 & 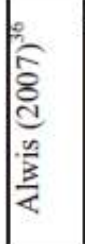 & 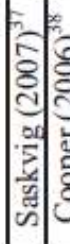 & 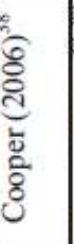 & 赵 & 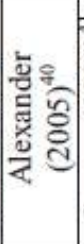 & 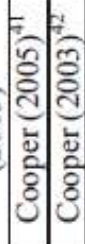 & 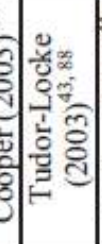 & 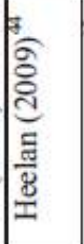 & 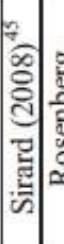 & 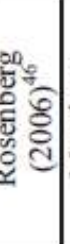 & 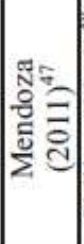 & 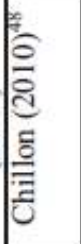 \\
\hline
\end{tabular}




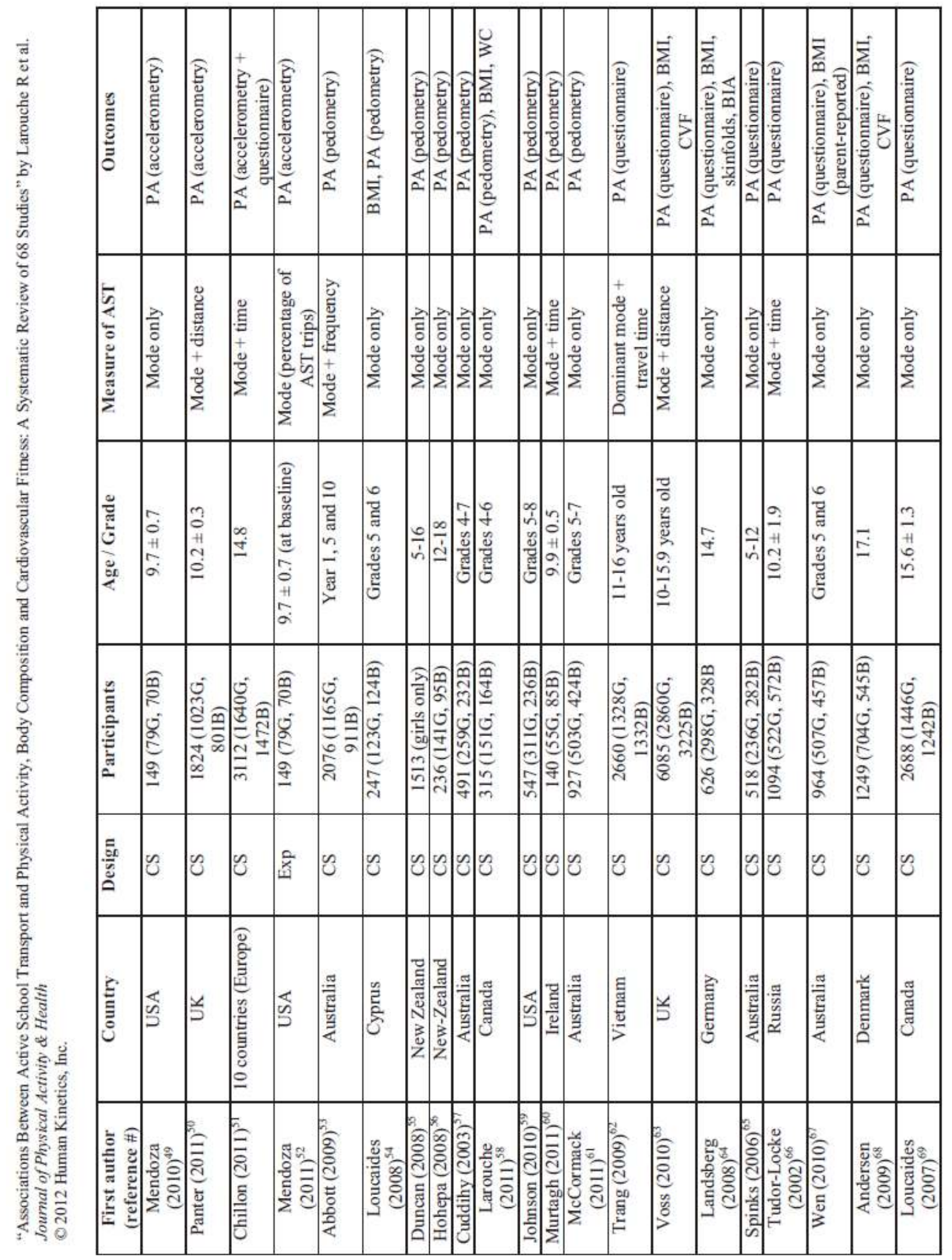




\begin{tabular}{|c|c|c|c|c|c|c|c|c|c|c|c|c|c|c|c|c|}
\hline |0ँ & 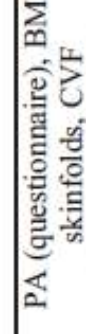 & & 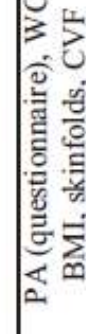 & & 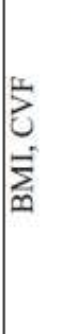 & 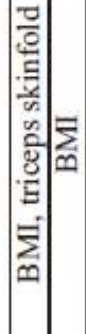 & & & 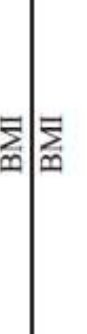 & 屏 & 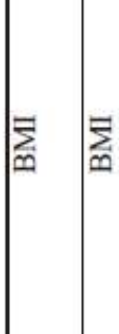 & : & 屏 & 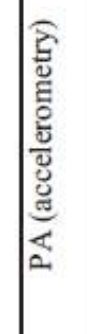 & & \\
\hline 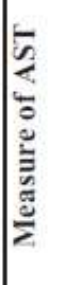 & 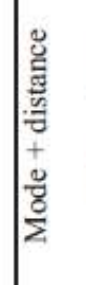 & 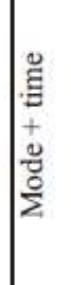 & 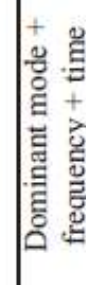 & & 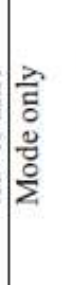 & & 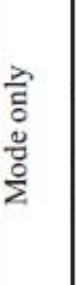 & 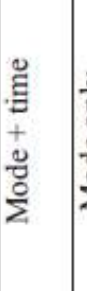 & : & 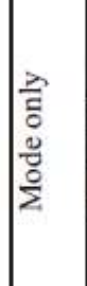 & 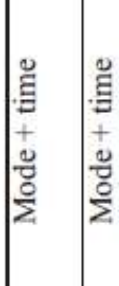 & $\frac{1}{3}$ & 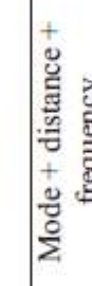 & 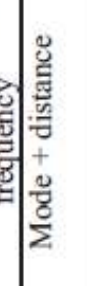 & 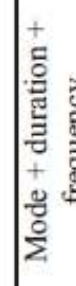 & \\
\hline 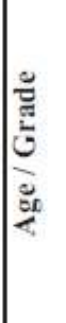 & $\begin{array}{l}\hat{b} \\
\hat{y} \\
\tilde{y} \\
\underline{0}\end{array}$ & 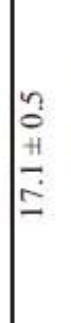 & 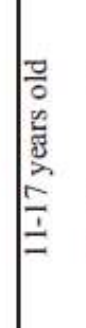 & 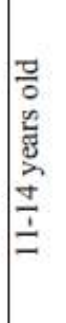 & 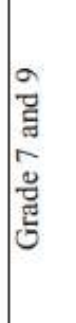 & & & 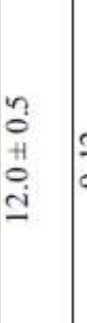 & 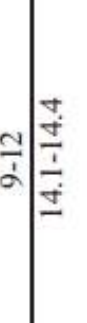 & 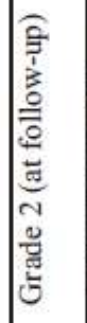 & 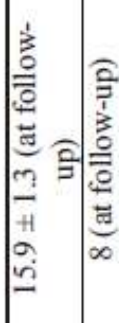 & 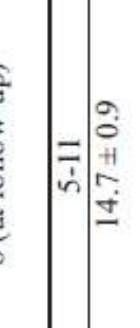 & 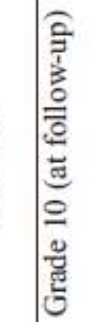 & $\frac{1}{2}$ & $\frac{n}{\dot{n}}$ & \\
\hline 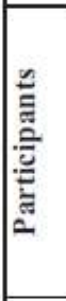 & 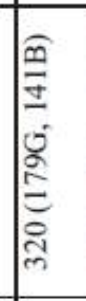 & 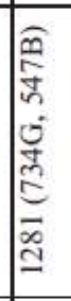 & 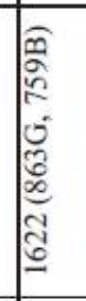 & & 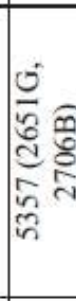 & & : & 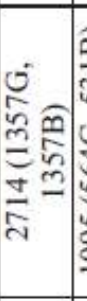 & 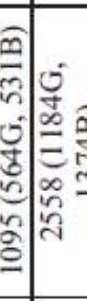 & 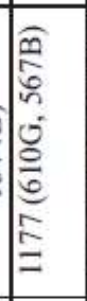 & 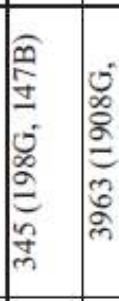 & ân & 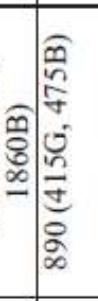 & 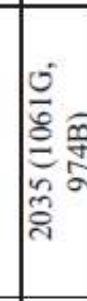 & مئ & - \\
\hline | & $£$ & 8 & 3 & 3 & 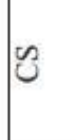 & & $y$ & c & 8 & 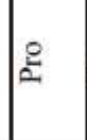 & $\cong$ & 10 & $\nsubseteq$ & 8 & 8 & 8 \\
\hline 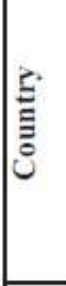 & 离 & $\mid$ & 咙 & $\underline{z}$ & $\infty$ & & ] & 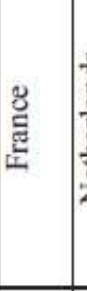 & 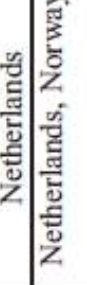 & 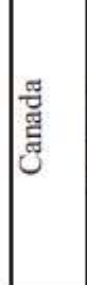 & 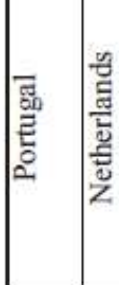 & $\frac{1}{4}$ & 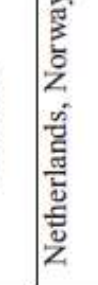 & 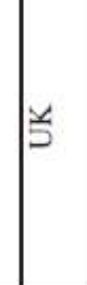 & $\because$ & $y$ \\
\hline & 站 & 命 & 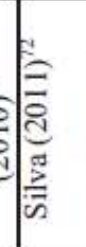 & 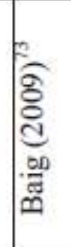 & 赵 & & 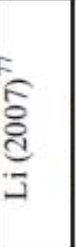 & 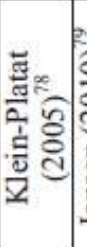 & 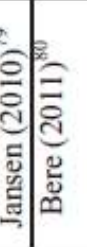 & 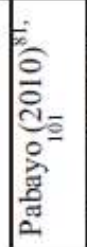 & 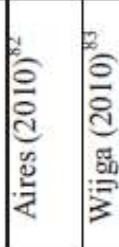 & 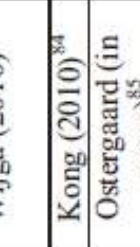 & 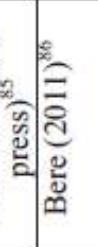 & 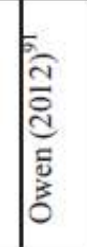 & 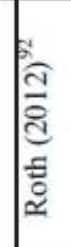 & . \\
\hline
\end{tabular}




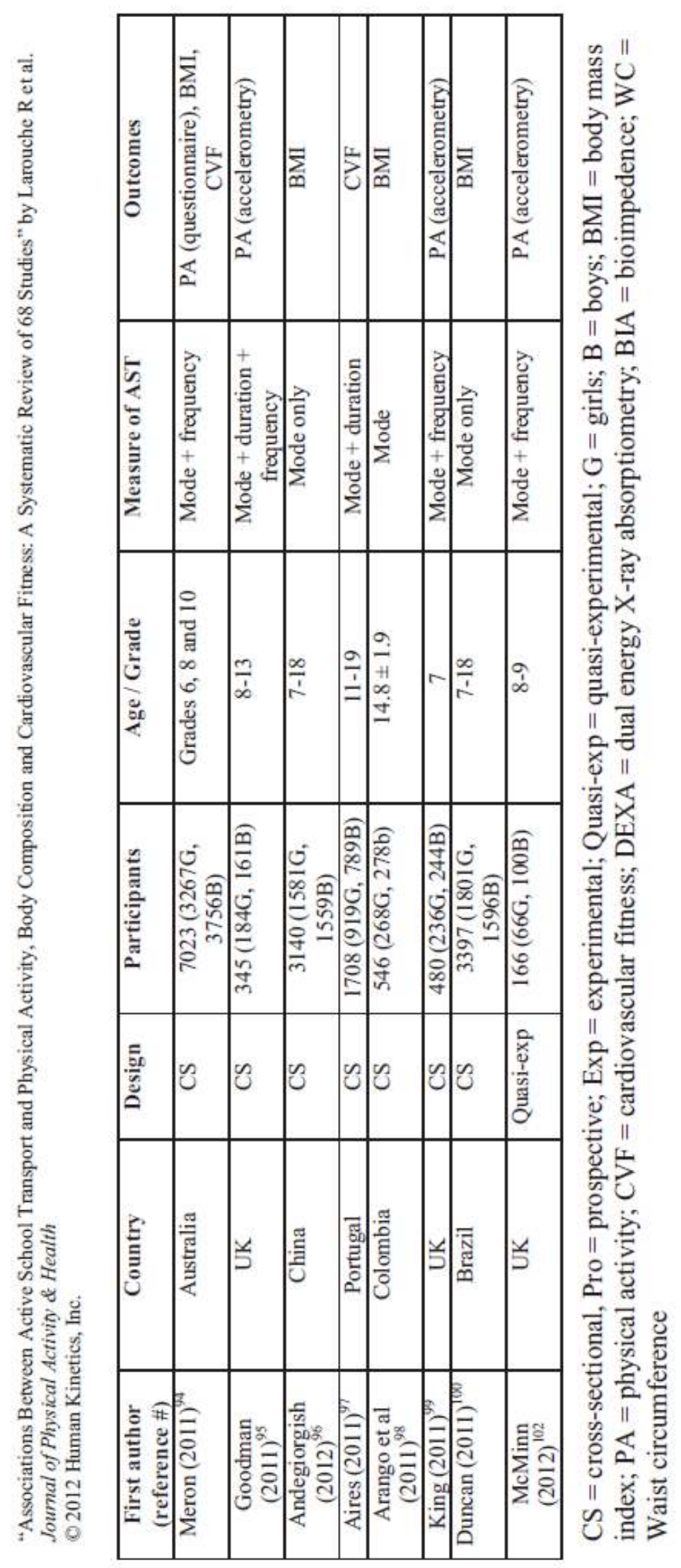




\begin{tabular}{|c|c|c|c|c|c|c|c|c|c|c|c|c|c|c|}
\hline 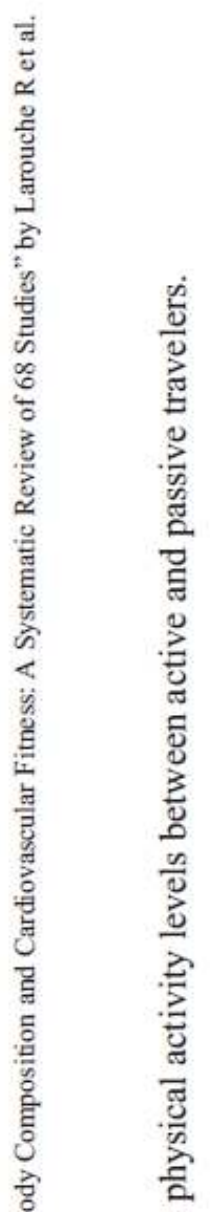 & 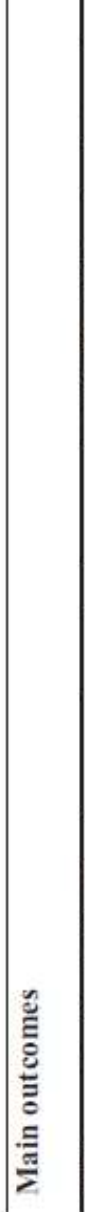 & 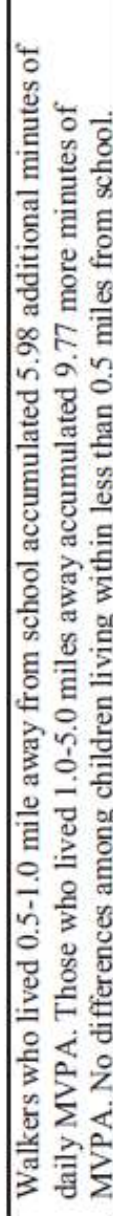 & 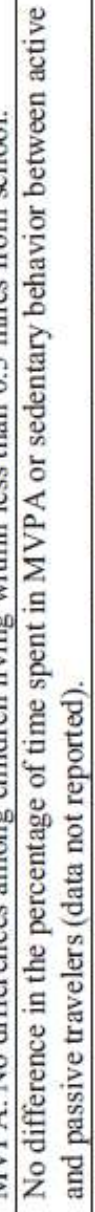 & 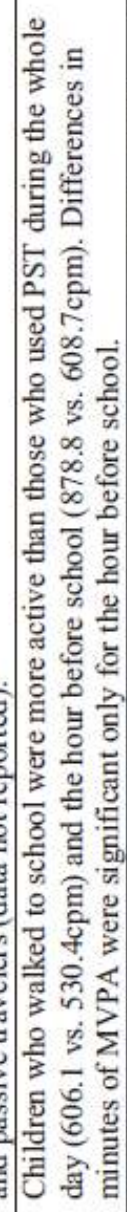 & 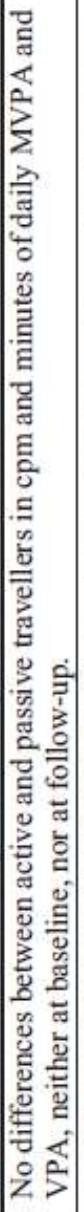 & 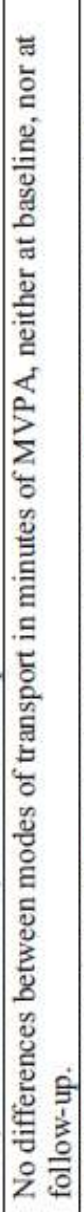 & 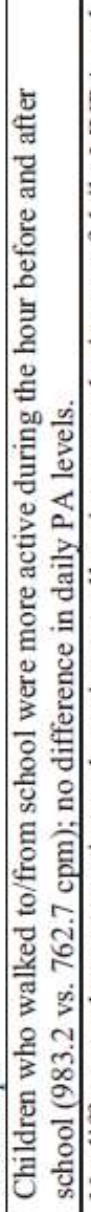 & 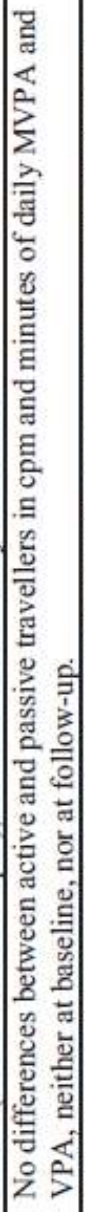 & 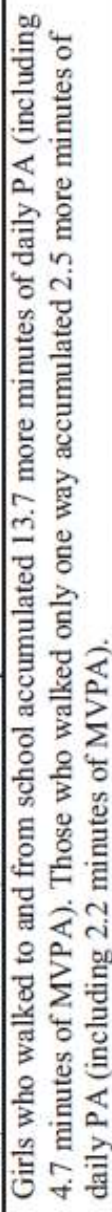 & 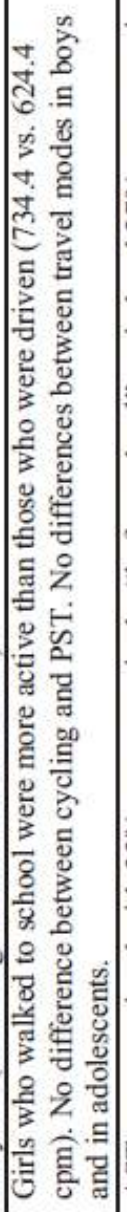 & 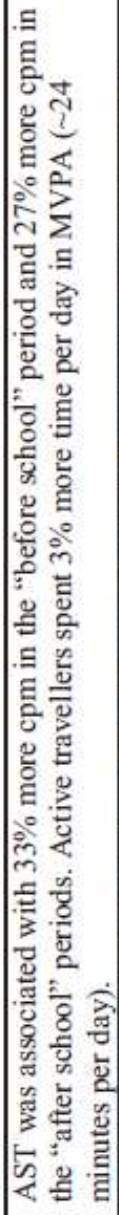 & 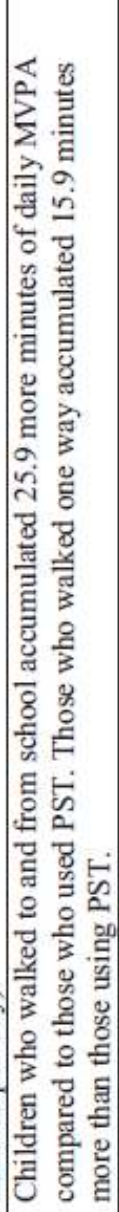 & 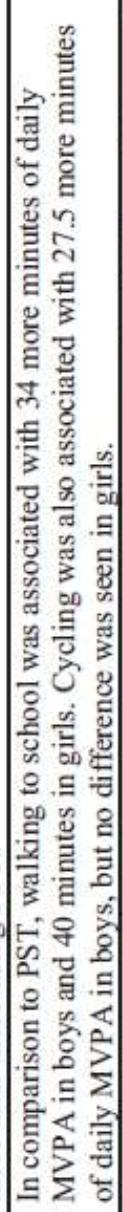 & 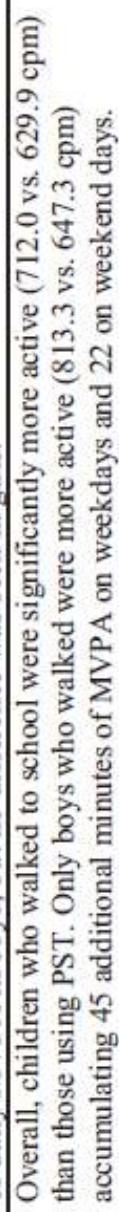 \\
\hline 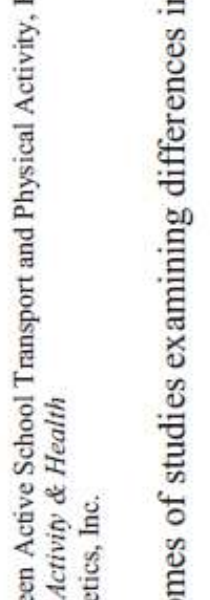 & 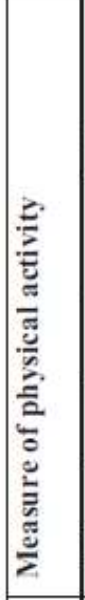 & 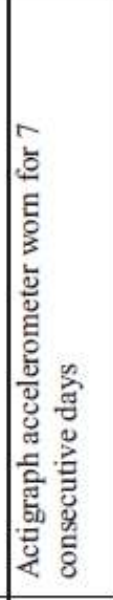 & 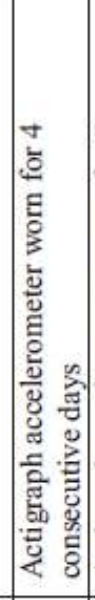 & 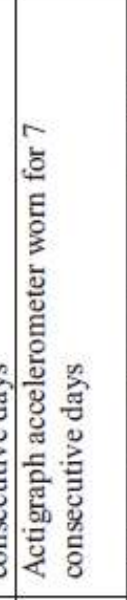 & 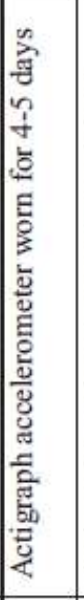 & $\bar{\S}$ & 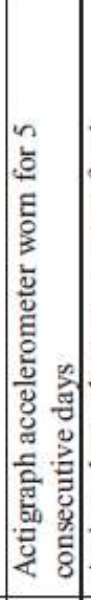 & 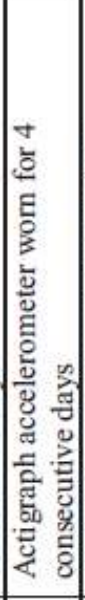 & 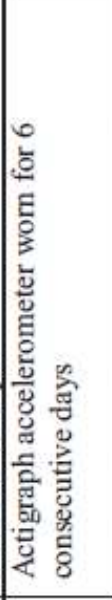 & E & 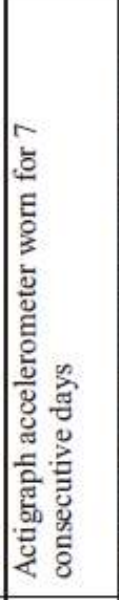 & 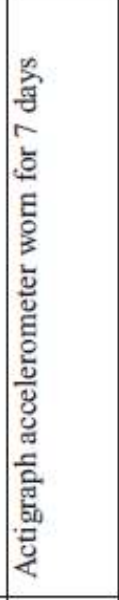 & ষ্ত & 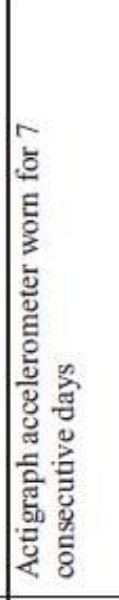 \\
\hline 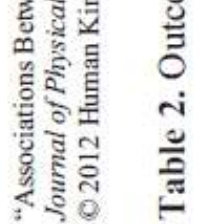 & 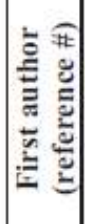 & 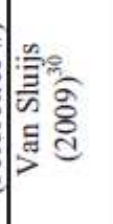 & 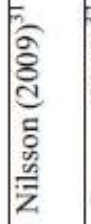 & 言 & 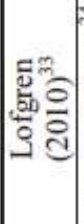 & & & 造 & \&్d & 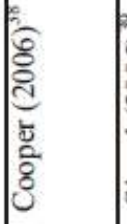 & dָ & है & 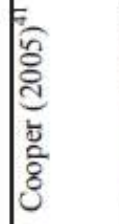 & \\
\hline
\end{tabular}




\begin{tabular}{|c|c|c|c|c|c|c|c|c|c|c|c|c|}
\hline 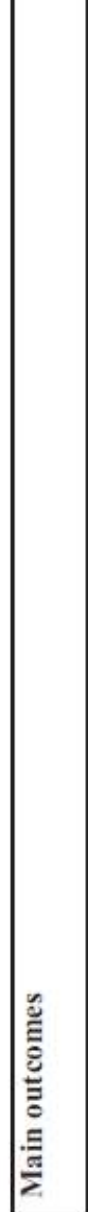 & 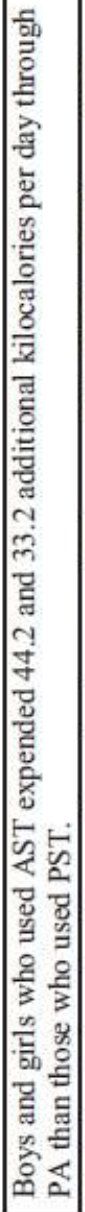 & 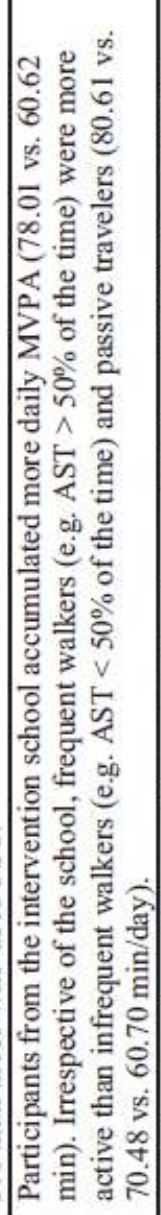 & 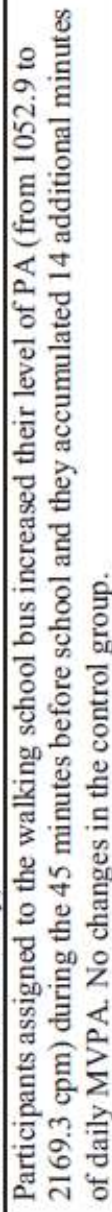 & 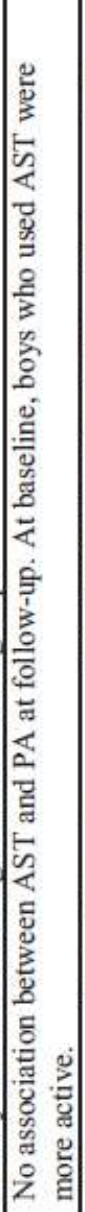 & 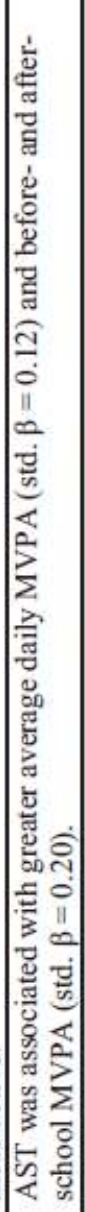 & 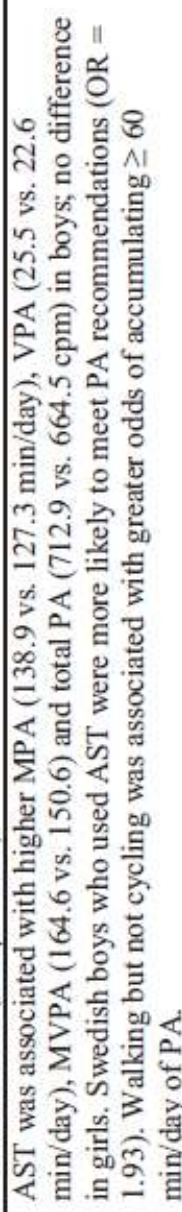 & 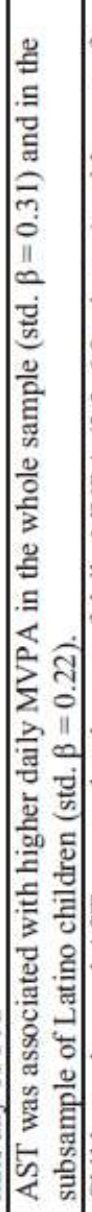 & 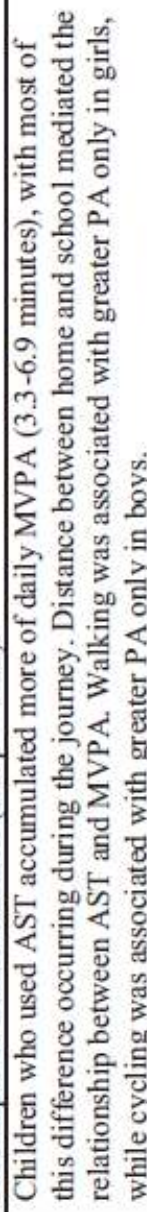 & 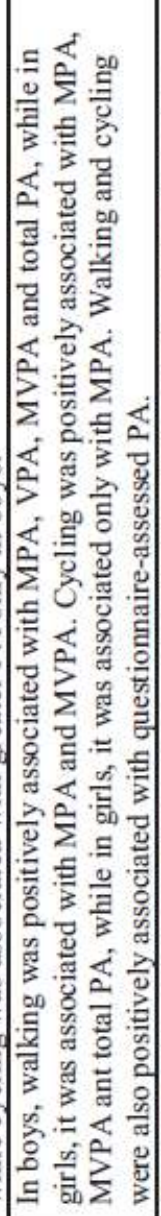 & 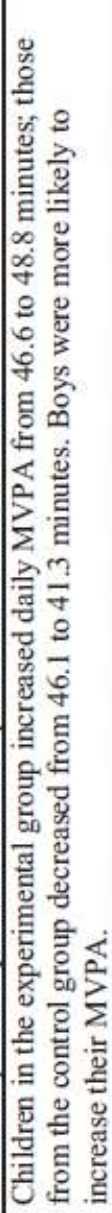 & 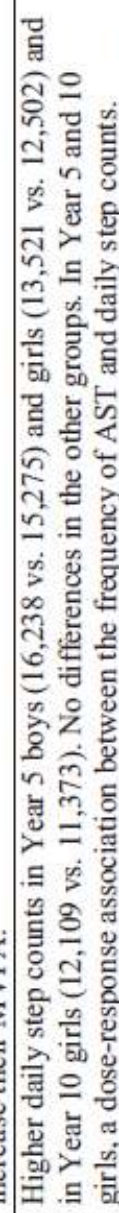 & 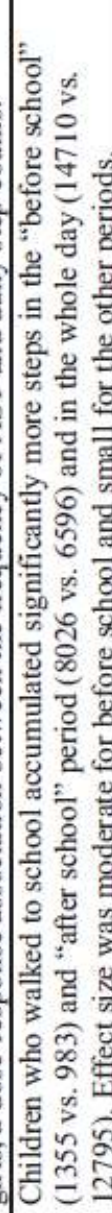 \\
\hline 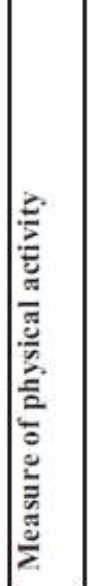 & 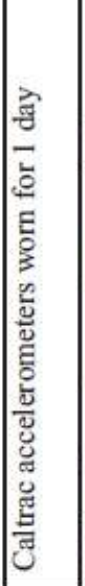 & 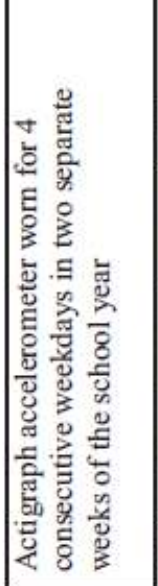 & 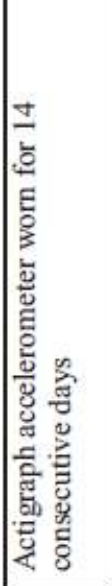 & 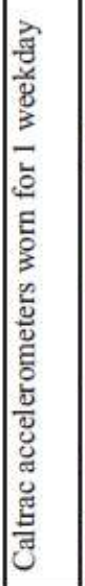 & 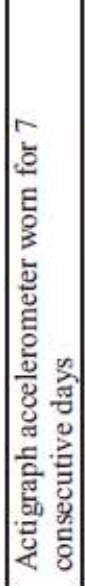 & 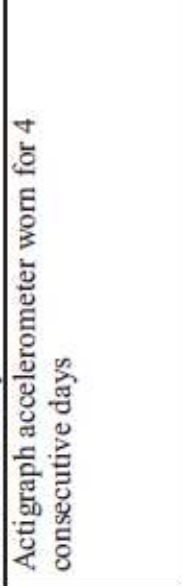 & 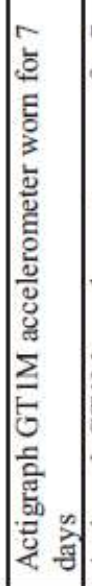 & 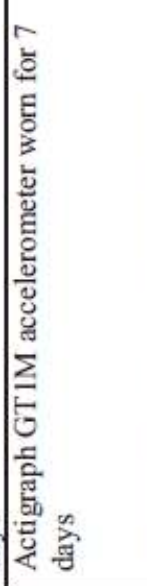 & 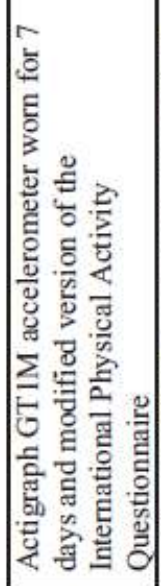 & 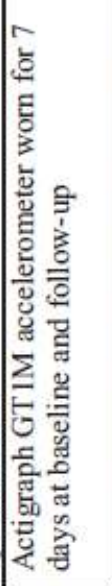 & 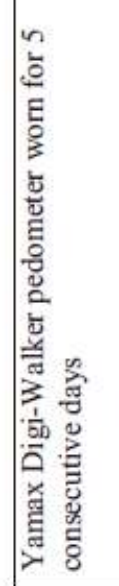 & 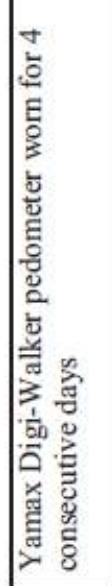 \\
\hline 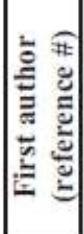 & 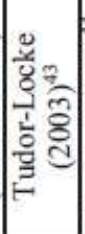 & 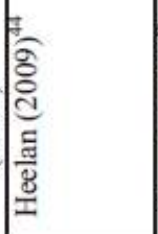 & 递 & 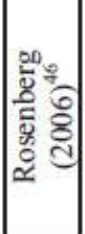 & 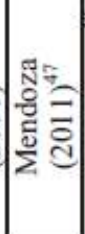 & 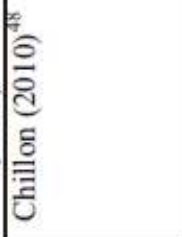 & 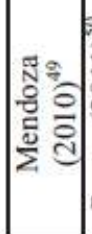 & 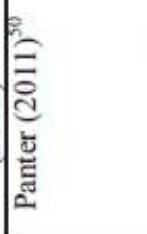 & 言 & 曹 & 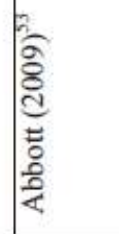 & 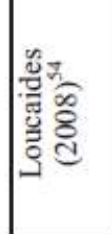 \\
\hline
\end{tabular}




\begin{tabular}{|c|c|c|c|c|c|c|c|c|c|c|c|c|c|c|c|c|}
\hline 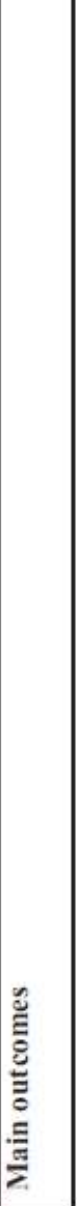 & 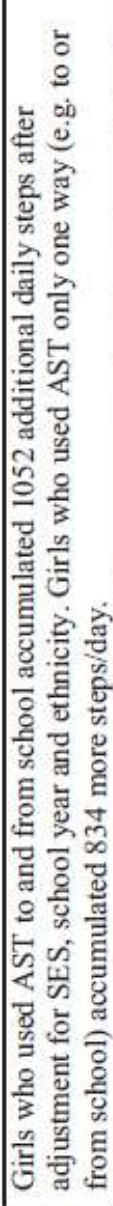 & 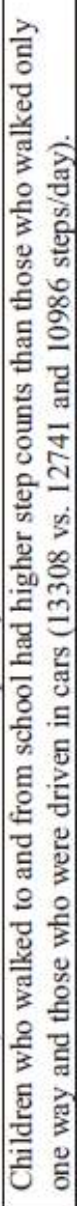 & 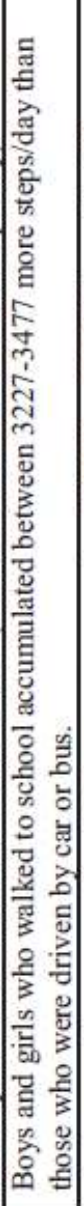 & 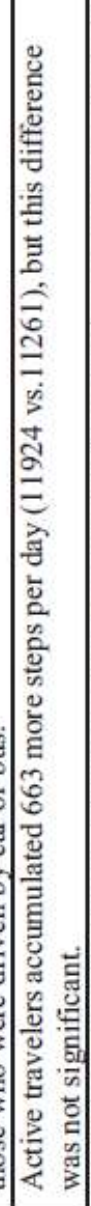 & 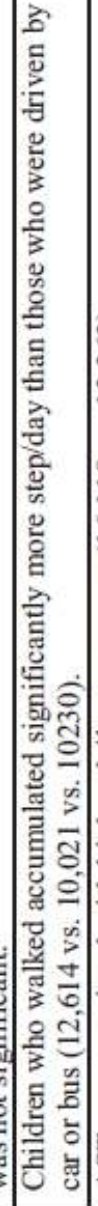 & 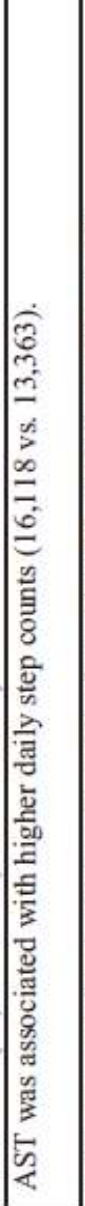 & 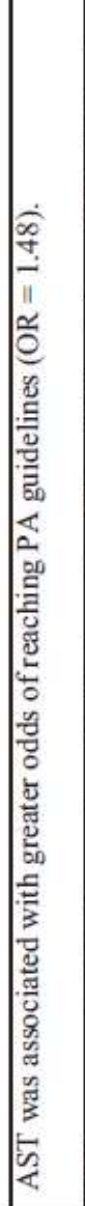 & 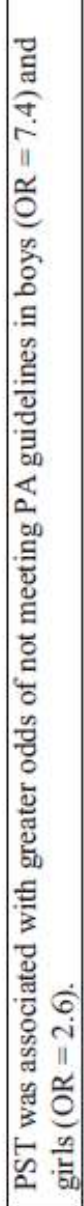 & 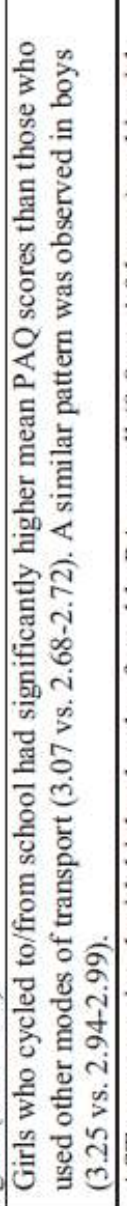 & 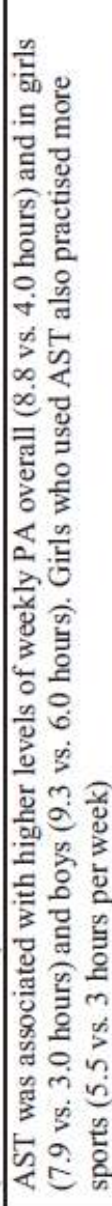 & 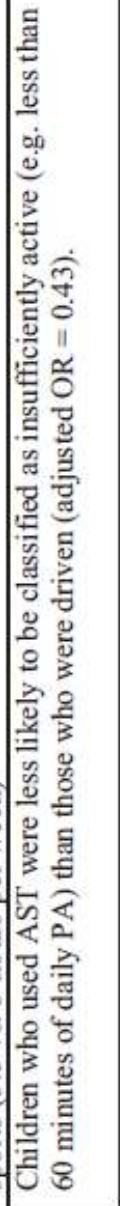 & 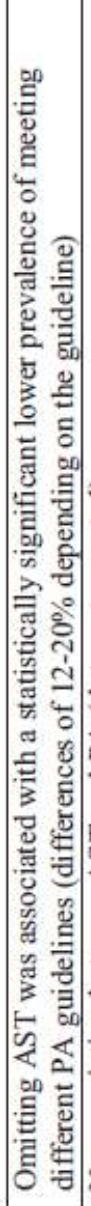 & & 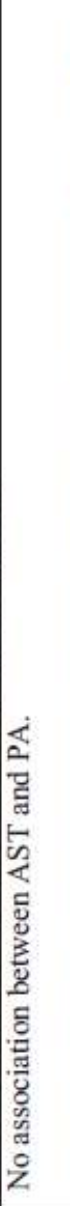 & 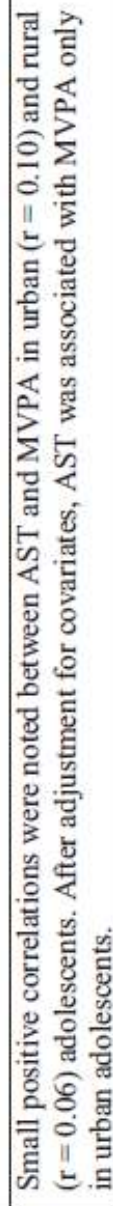 & 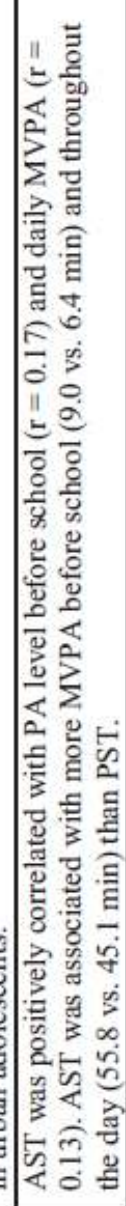 \\
\hline 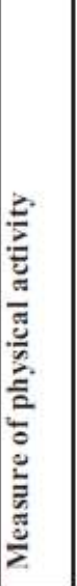 & 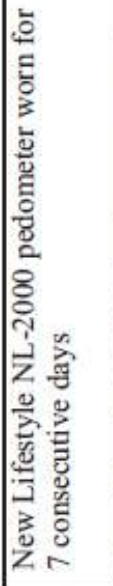 & 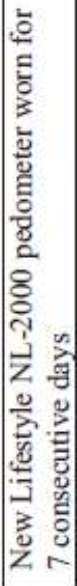 & 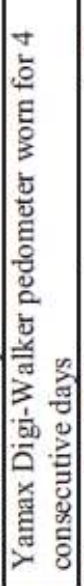 & 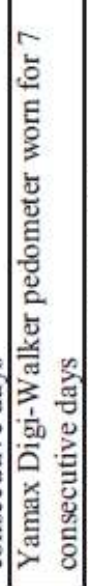 & 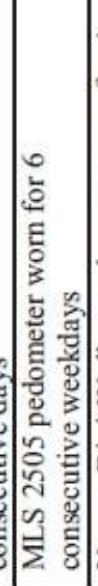 & 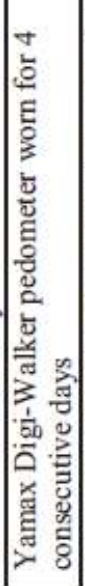 & 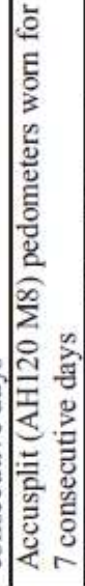 & 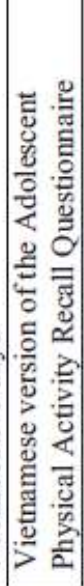 & 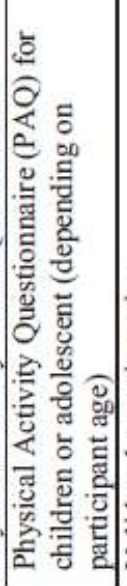 & 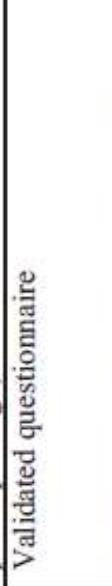 & 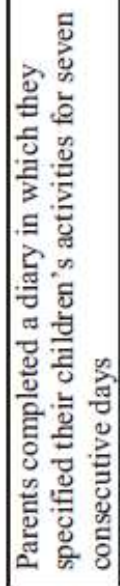 & 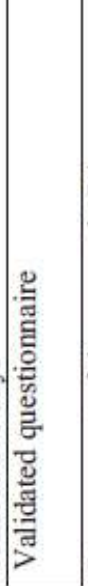 & & 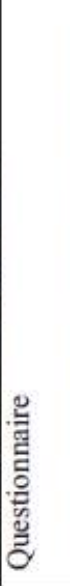 & 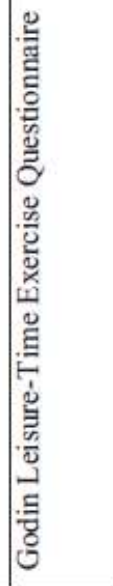 & 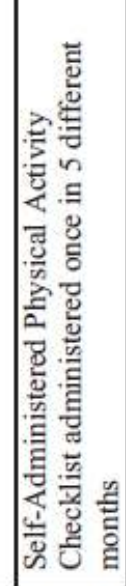 \\
\hline 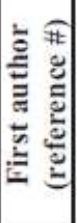 & 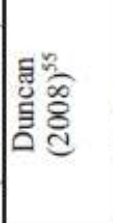 & 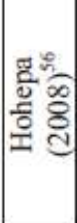 & 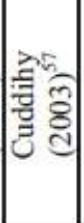 & 吾 & 送 & 至 & $\mid \begin{array}{ll}0 \\
:\end{array}$ & $\frac{5}{50}$ & 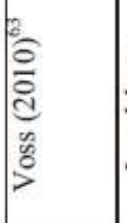 & 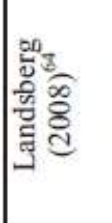 & 1 & 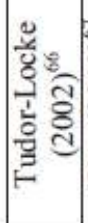 & 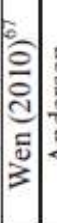 & 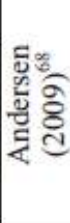 & 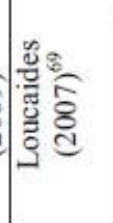 & 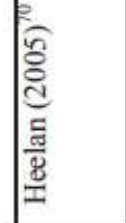 \\
\hline
\end{tabular}




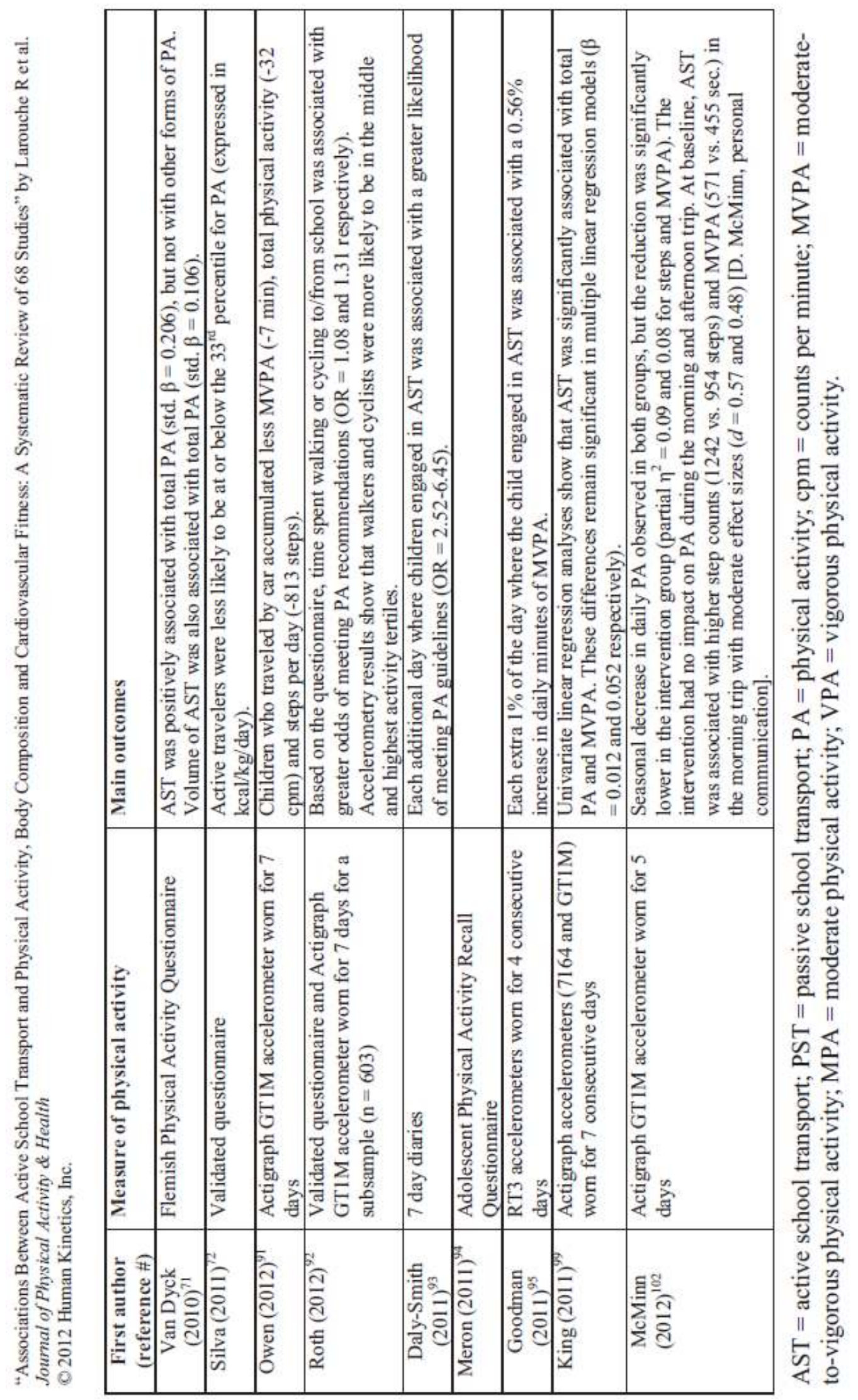




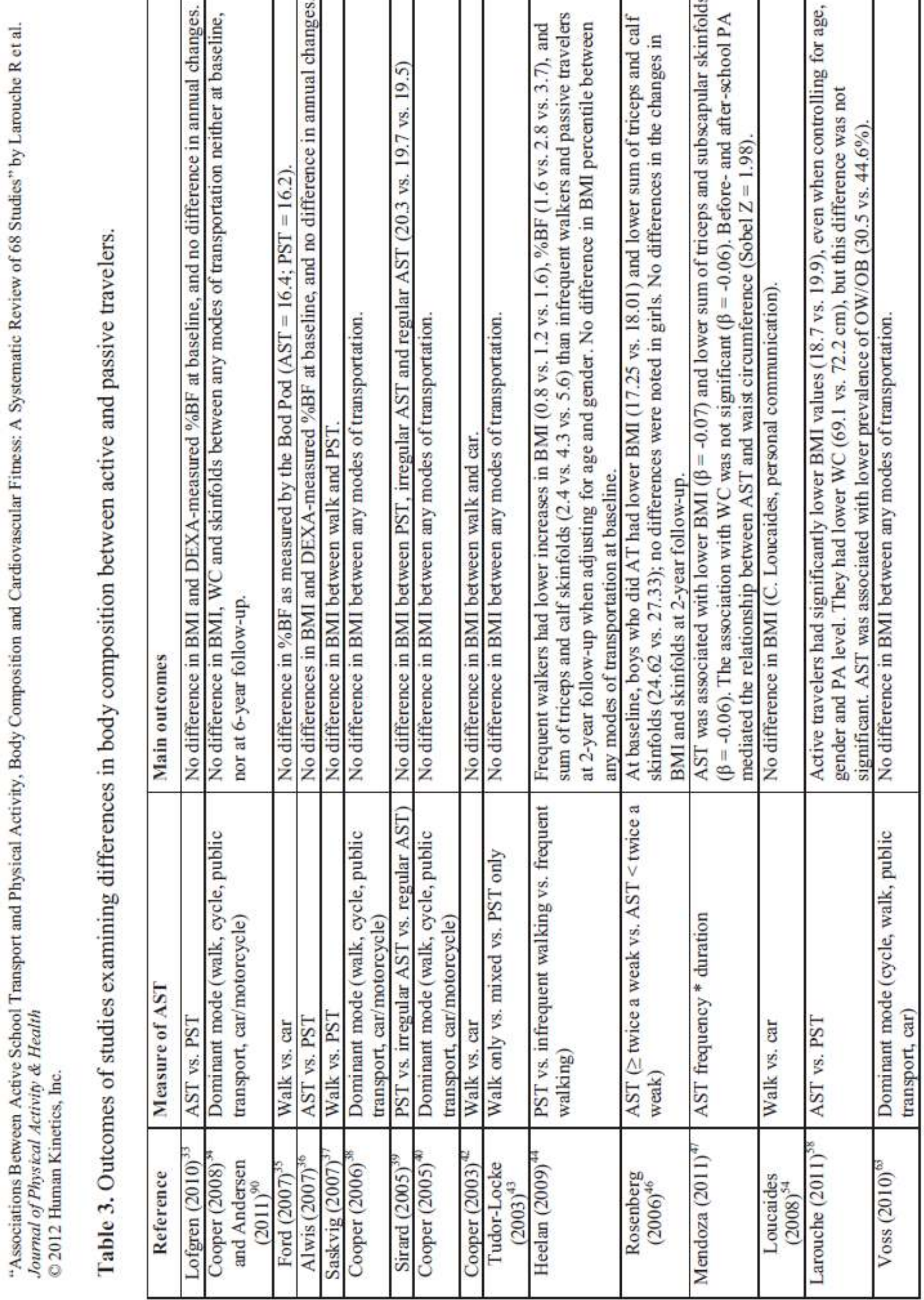




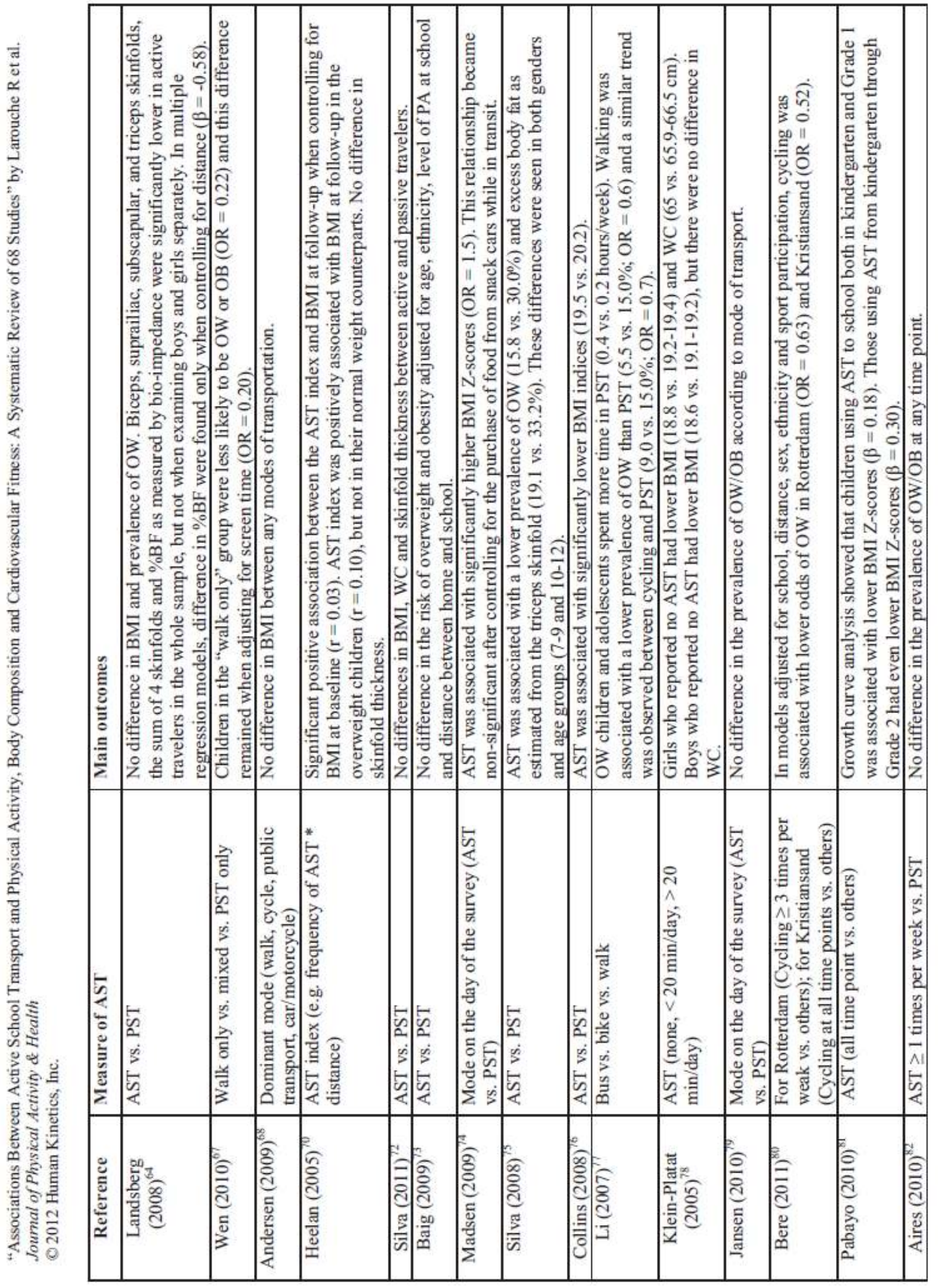




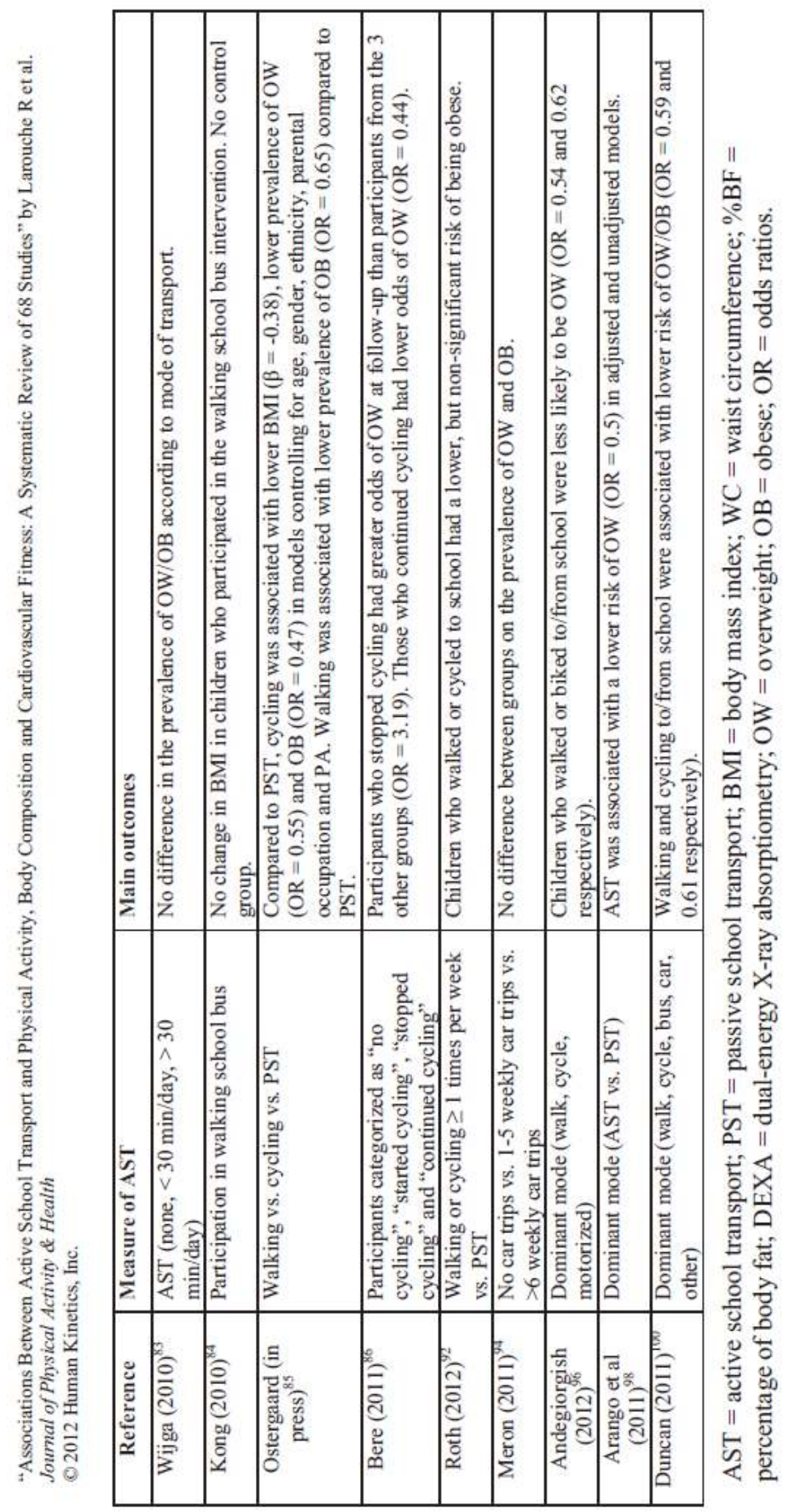




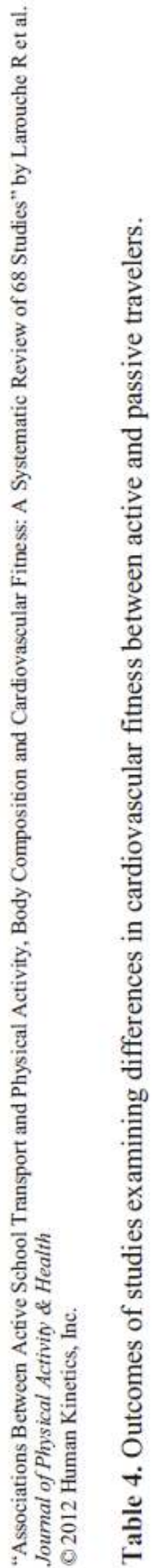

\begin{tabular}{|c|c|c|c|c|c|c|c|c|c|c|}
\hline & 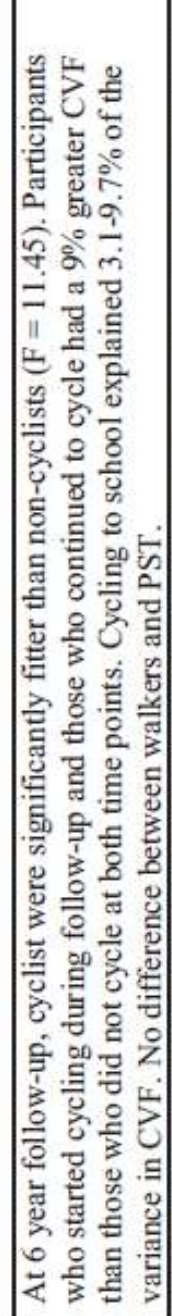 & 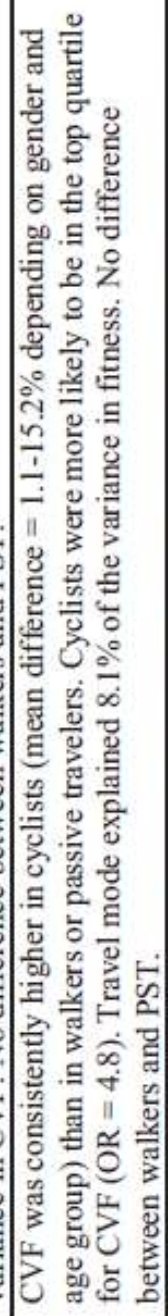 & 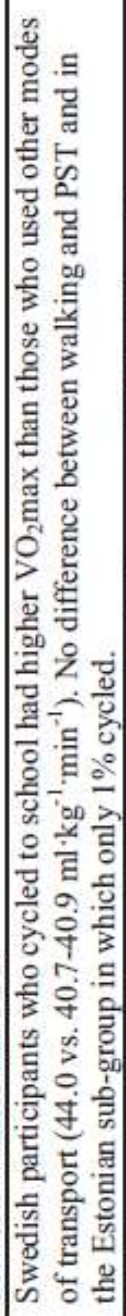 & 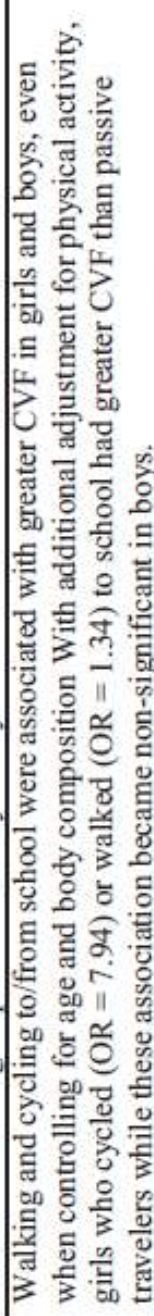 & 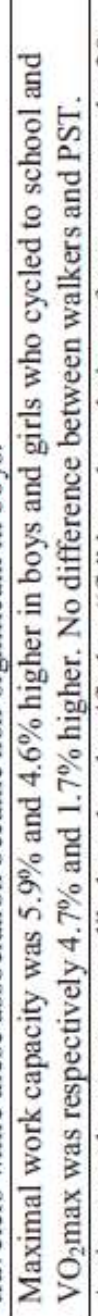 & 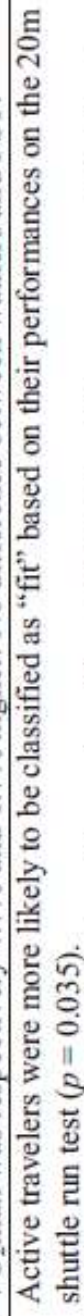 & 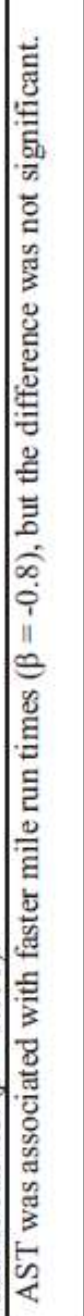 & 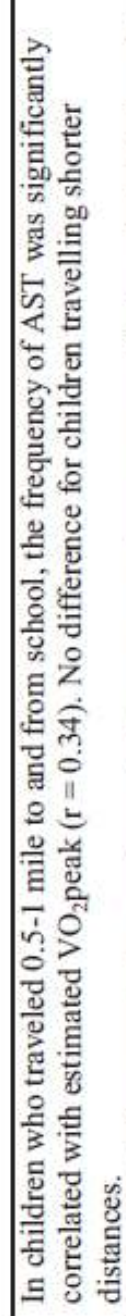 & 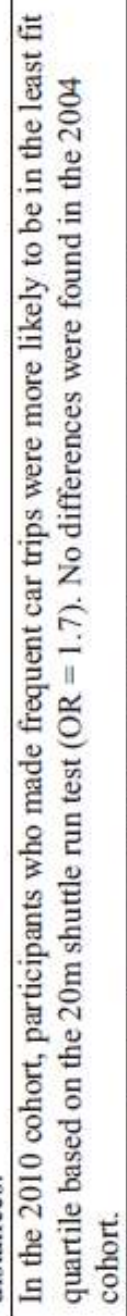 & 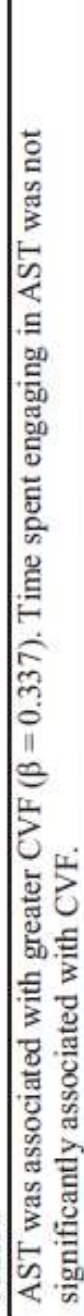 \\
\hline 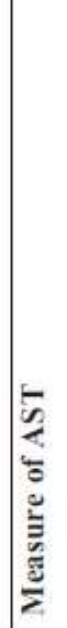 & 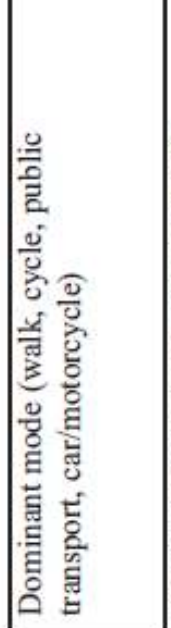 & 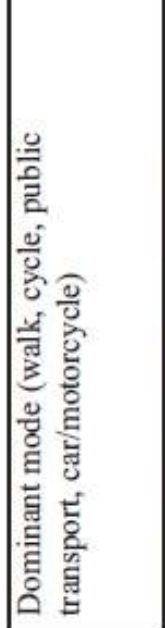 & 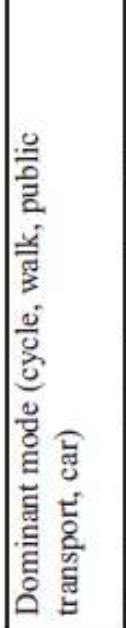 & 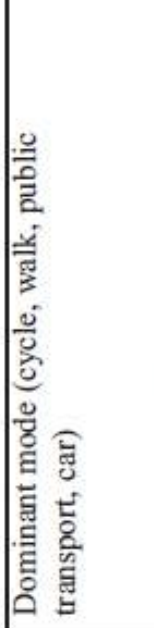 & 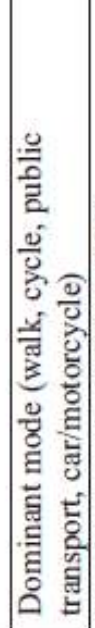 & 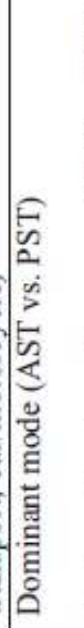 & 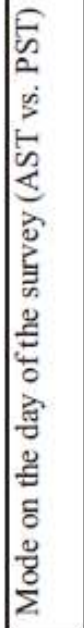 & 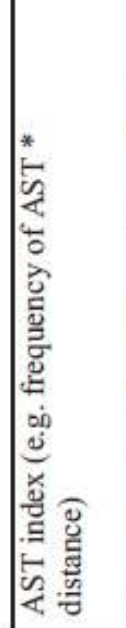 & 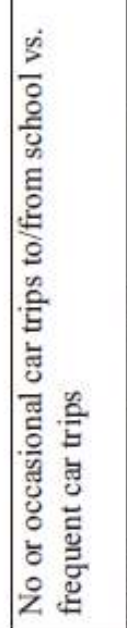 & 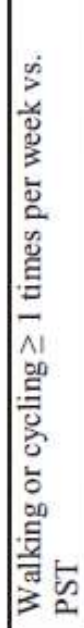 \\
\hline हूँّ & 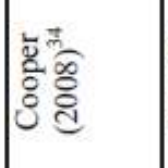 & 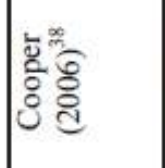 & 을 & $p \stackrel{8}{\circ} \stackrel{0}{\circ}$ & 递 & $\frac{\pi}{2}$ & 4. & $\begin{array}{ll}\frac{\pi}{9} & 8 \\
\pm & 0 \\
1 & 0\end{array}$ & 은 & $\Xi$ \\
\hline
\end{tabular}




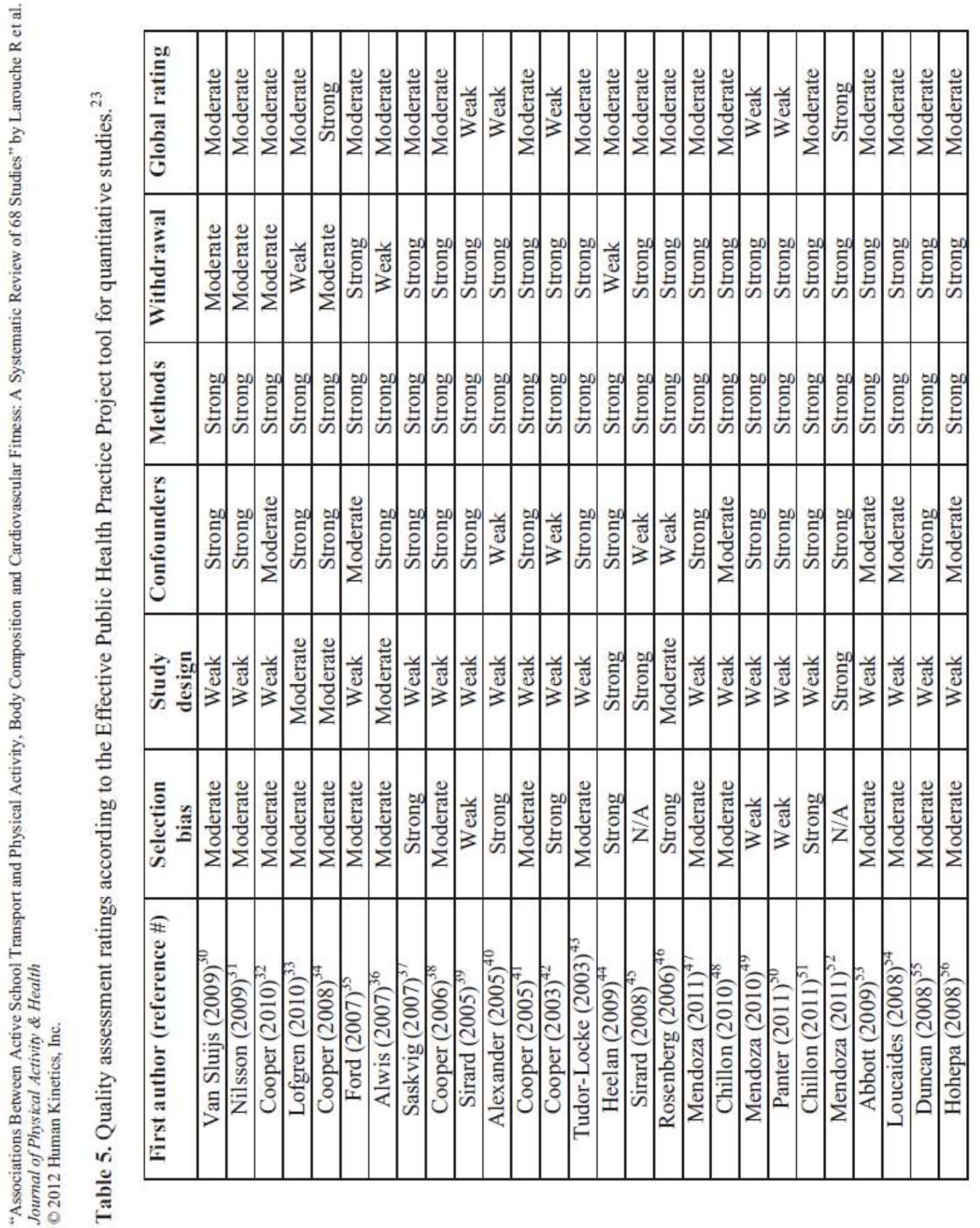




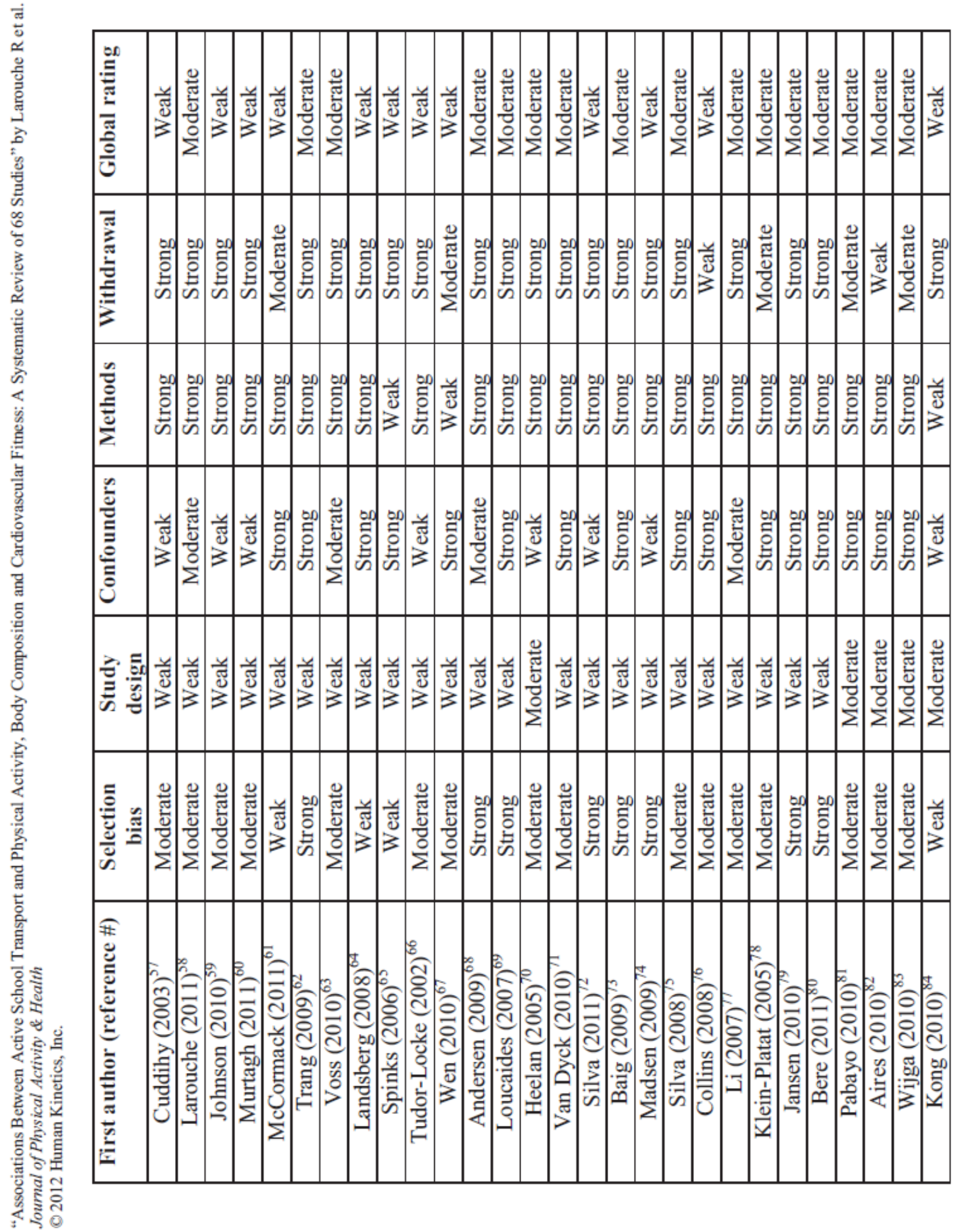




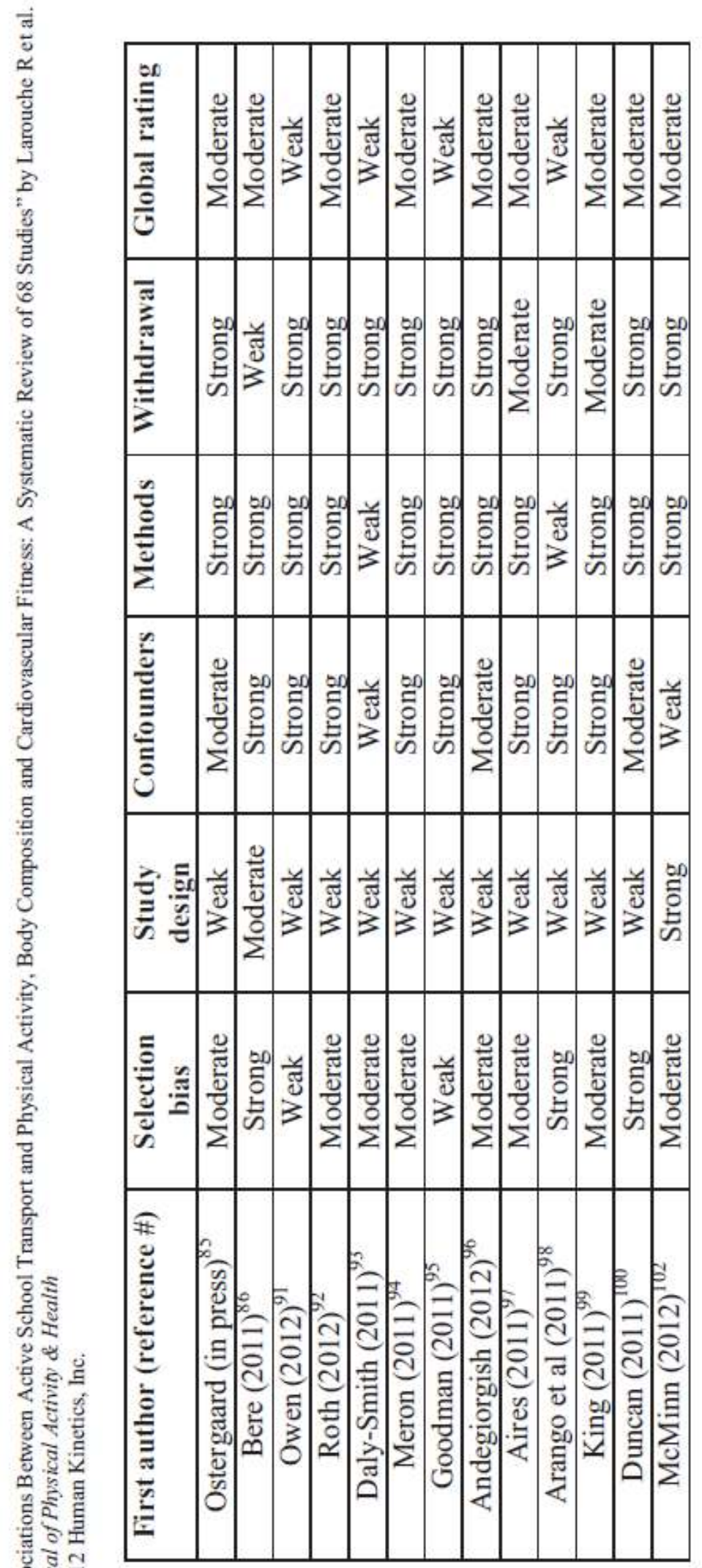

II 
"Associations Between Active School Transport and Physical Activity, Body Composition and Cardiovascular Fitness: A Systematic Review of 68 Studies" by Larouche R et al.

Joumal of Physical Activity \& Health

○ 2012 Human Kinetics, Inc.

Figure 1. Flow of articles through the search process

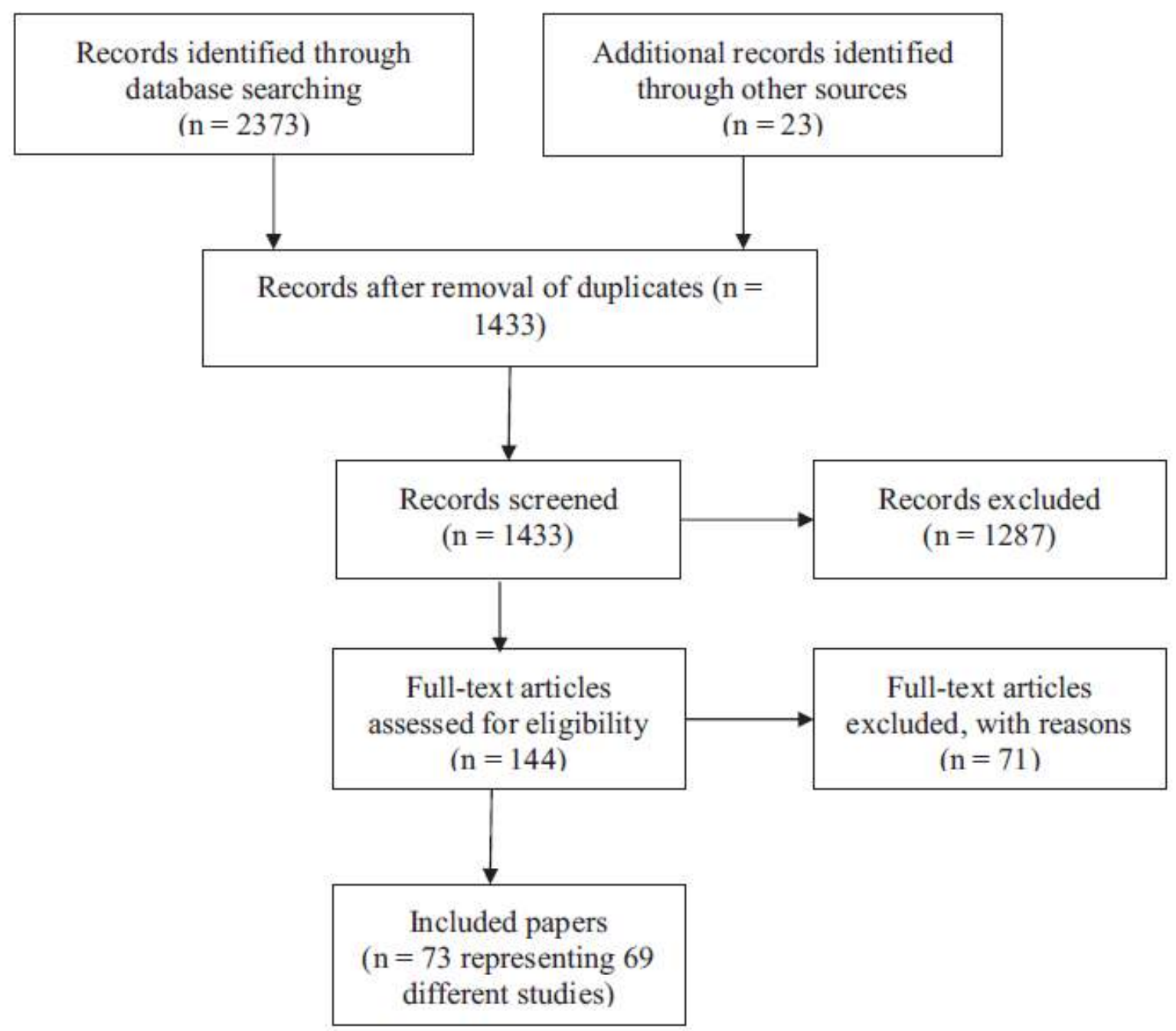




\section{Chapter 3. Manuscript II: "Relationship between active school transport and body mass index in Grades 4-to-6 children"}

Authors: Richard Larouche, Meghann Lloyd, Emily Knight \& Mark S. Tremblay

Status: Published in Pediatric Exercise Science (2011), Vol. 23, p. 322-330.

Presented at: $2^{\text {nd }}$ Joint Meeting of the North American Society for Pediatric Exercise Medicine and the Pediatric Work Physiology Group. Niagara-on-the-Lake, ON (2010). 


\title{
Relationship Between Active School Transport and Body Mass Index in Grades- 4-to-6 Children
}

\author{
Richard Larouche, Meghann Lloyd, Emily Knight, \\ and Mark S. Tremblay \\ Children's Hospital of Eastern Ontario Research Institute
}

\begin{abstract}
The current investigation assessed the impact of active school transportation (AST) on average daily step counts, body mass index (BMI) and waist circumference in 315 children in Grades $4-6$ who participated to Cycle 2 of the Canadian Assessment of Physical Literacy (CAPL) pilot testing. T-tests revealed a significant association between AST and lower BMI values $\left(18.7 \pm 3.3 \mathrm{vs.} 19.9 \pm 3.8 \mathrm{~kg} / \mathrm{m}^{2}\right)$. The active commuters accumulated an average of 662 more steps per day, and their waist circumference was lower by an average of $3.1 \mathrm{~cm}$, but these differences were not statistically significant. ANCOVA analyses controlling for age and step counts, found trends toward lower BMI and waist circumference values among the active commuters. These results suggest that AST may be a valid strategy to prevent childhood obesity: further research is needed to determine more precisely the impact of AST on body composition, and the direction of the relationship.
\end{abstract}

The prevalence of childhood overweight and obesity has increased rapidly in many countries and it is now considered a pandemic (43). Recent data from the Canadian Health Measures Survey illustrate that the rising prevalence of obesity occurred in conjunction with a sevenfold increase in the prevalence of elevated waist circumference in Canadian youth between 1981 and 2007-2009 (25). Further, according to the 2010 Active Healthy Kids Canada report card on physical activity for children and youth (2). only a small proportion of Canadian children and adolescents meet either the 60 or the $90 \mathrm{~min}$ of daily moderate to vigorous physical activity (MVPA) guidelines (22.36). Insufficient physical activity among children and youth has been linked to several health problems such as impaired glucose tolerance and cardiovascular disease risk factors $(4,24)$.

To alleviate these societal trends, researchers have traditionally focused on increasing leisure-time physical activity as a form of intervention. However, emerging evidence suggests that, in isolation, increasing leisure-time physical activity may be an insufficient approach to prevent and/or treat obesity and its complica-

Larouche, Lloyd, Knight, and Tremblay are with the Healthy Active Living and Obesity Research Group, Children's Hospital of Eastem Ontario Research Institute, University of Otawa, Ontario, Canada. 
tions $(5,37)$. Such observations have led to the emergence of research focusing on the concept of "active living", within which active school transportation (AST) represents a key component for children $(34,35)$.

In a recent systematic review, Faulkner and colleagues (20) reported that in 11 of 13 studies, children who engaged in AST had higher levels of objectively measured physical activity on a daily basis. However, based on the existing data, these authors could not draw a strong conclusion on the relationship between AST and body weight. The purpose of the current study was to examine the relationship between AST, daily physical activity and body composition variables (e.g., body mass index [BMI] and waist circumference) among Eastern Ontario children in grades 4-6 who participated in pilot testing of the Canadian Assessment of Physical Literacy (CAPL; 27,38),

\section{Methods}

The CAPL is a contemporary assessment tool that is still in the development process. It is being developed with the goal of being a valid, reliable, feasible and informative tool to assist in the assessment of physical literacy in children. The CAPL assesses the four domains of physical literacy: fundamental motor skills, physical activity behavior, physical fitness, and knowledge, awareness and understanding $(26,38)$.

\section{Participants}

Research ethics approval was obtained from the researchers' institution as well as two local participating school boards ( 1 urban, 1 rural). Participation was completely voluntary and written informed consent was obtained from all parents/guardians. In addition written assent was obtained from each participant. Data for the current study are from the second cycle of pilot testing (cycle I was primarily feasibility testing). In cycle 2, 364 children from grades 4-6 participated and were asked to report their primary mode(s) of transportation to and from school in both the Fall/ Winter and the Spring/Summer seasons as part of the knowledge, awareness and understanding assessment module. Specifically, the children were asked "Please circle how you get to school most of the time in the Fall/Winter (during cold or snowy weather)" and "in the Spring/Summer (during nice weather)?"

Forty-nine participants did not answer the questionnaire and/or the question related to AST. Thus, the data from 315 children with a mean age of $10.1 \pm 0.9$ years were included in the current analyses. T-tests revealed no difference between these children and those that did not report their mode of transportation for age, sex, BMI and waist circumference. However, the excluded participants accumulated an average of 1346 less steps per day $(\mathrm{t}=2.06 ; p=.04)$.

\section{Procedure}

The participants were instructed to wear a pedometer (Digi-Walker \$W200) for 7 consecutive days and to complete a daily log recording their daily step counts as well as the time the pedometer was worn during waking hours. The same pedometer and data collection procedures were used by the Canadian Fitness and 
Lifestyle Research Institute in the annual nationally representative CANPLAY study (15). The assessment of body composition was conducted by a Certified Exercise Physiologist (details of this certification can be found at http://www. csep.ca/english/view.asp? $x=739$ ), according to the Canadian Physical Activity, Fitness \& Lifestyle Approach protocol (9). Height was measured to the nearest $0.1 \mathrm{~cm}$ and without footwear using a portable stadiometer (SECA: Hamburg, Germany). Weight was measured wearing light clothing and without footwear and to the nearest $0.1 \mathrm{~kg}$ with a digital scale (A\&D Medical: Milpitas, CA.). Waist circumference was measured to the nearest $0.5 \mathrm{~cm}$ at the top of the iliac crest. and at the end of a normal expiration. Data collection was performed during the Fall of 2009 and Winter 2010.

\section{Data Treatment and Analysis}

The raw pedometry data were screened based on established criteria including 1) Between 1000 and 30000 steps/day $(31,40) ; 2)$ At least 10 hr of data/day $(12,19)$ and 3) At least three days of valid data (e.g., meeting the daily wear threshold values; 40). The days where these criteria were not met were removed and replaced by the average daily step counts for each participant, as recommended by Rowe and colleagues (32). Application of these criteria resulted in the removal of 13 person-days for outlying number of steps/day and 84 person-days for insufficient wear time. The pedometry data from five participants was excluded because they had less than three days of valid measurement. In total, acceptable pedometry data were available for 262 participants for a total of 1642 person-days.

Based on their self-reported modes of transportation to and from school, the participants were classified into three groups: passive commuters, active commuters, and multiple modes (i.e,, a combination of active and passive modes of transportation such as walking and being driven). ANOVAs were performed to assess differences between groups for average daily step counts. BMI and waist circumference. The Bonferroni post hoc test was used given the differences in the number of participants in each group.

As illustrated in Table 1, only 27 participants ( $8.6 \%$ of the total sample) reported multiple modes of transportation and variability between subjects was very high among this subgroup for the outcome variables. In addition. the ANOVAs revealed no significant differences between those who reported multiple modes of transportation and the other subgroups. Thus, subsequent analyses were performed only between the active and passive commuters using $t$ tests. Chi-square analyses were also used to compare the rates of overweight and obesity based on the International Obesity Task Force (IOTF) classification (11). Finally, ANCOVAs were performed to compare the mean values for BMI and waist circumference while controlling for physical activity level (average daily step counts) and age. The statistical analyses were performed with the PASW Statistics 18 software (SPSS inc,. Chicago, Il.) and the level of significance was set at $p<.05$. For the ANCOVAs, the estimated marginal means are presented with $95 \%$ confidence intervals (CI). Normal distribution of the outcome variables (BMI. waist circumference and steps/day) could not be rejected based on the skewness and kurtosis of the distribution. Levene's test for equality of variance was performed with the $t$ tests and all $p$ values were $\geq 0.05$. 


\section{Table 1 Descriptive Characteristics of the Sample}

\begin{tabular}{lccc}
\hline Characteristics & $\begin{array}{c}\text { Active } \\
\text { commuters }\end{array}$ & $\begin{array}{c}\text { Passive } \\
\text { commuters }\end{array}$ & $\begin{array}{c}\text { Multiple } \\
\text { modes }\end{array}$ \\
\hline Number of participants & 66 & 222 & 27 \\
Age (years) & $10.2 \pm 0.9$ & $10.1 \pm 0.8$ & $10.3 \pm 0.8$ \\
\% Girls & 48.5 & 48.6 & 40.7 \\
Step counts (steps per day) & $11924 \pm 3776$ & $11261 \pm 3107$ & $10641 \pm 3664$ \\
BMI $\left(\mathrm{kg}^{2} \mathrm{~m}^{2}\right)$ & $18.7 \pm 3.3$ & $19.9 \pm 3.8^{\circ}$ & $19.6 \pm 4.4$ \\
\% Overweight & 30.5 & $44.6^{*}$ & 33.0 \\
\% Obese & 6.8 & 14.2 & 12.5 \\
Waist circumference $(\mathrm{cm})$ & $69.1 \pm 9.6$ & $72.2 \pm 11.9$ & $74.1 \pm 13.3$ \\
\hline
\end{tabular}

N.B. Data are presented based on FallWinter modes of transportation. The prevalence of overweight and obesity is based on the International Obesity Task Force's thresholds (11). Asterisks illustrate significant differences between active and passive commuters $(p<.05)$.

\section{Results}

The prevalence of AST in this sample was $21.0 \%$ and $30.9 \%$ in the Fall/Winter and Spring/Summer seasons respectively. A Spearman correlation of $0.75(p<.01)$ indicates a high stability in mode of transportation throughout the year. Therefore, because the data on the outcome variables (physical activity and anthropometric measures) were collected in the Fall, only the mode of transportation reported for the Fall/Winter seasons was included in the analyses.

T-test results indicate that the active commuters had significantly lower BMI values $\left(18.7 \pm 3.3 \mathrm{vs} .19 .9 \pm 3.8 \mathrm{~kg} / \mathrm{m}^{2} ; p=04\right)$ and they had a lower prevalence of overweight and obesity than the passive commuters $\left(30.5 \mathrm{vs} .44 .6 \% ; \mathrm{X}^{2}=3.75 ; p\right.$ $=, 05$ ). Moreover. the prevalence of obesity was more than two times lower among the active commuters, although this difference is not statistically significant $(6.8 \%$ vs. $14.2 \% ; \mathrm{X}^{2}=2.31 ; p=13$ ). The active commuters accumulated an average of 662 more steps per day, but this difference was not significant (11 924 vs. 11262 steps: $p=.23$ ). In addition. the average waist circumference was lower by $3.1 \mathrm{~cm}$ in the active commuters in comparison with the passive commuters $(69.1 \pm 9.6 \mathrm{vs}$. $72.2 \pm 11.9 \mathrm{~cm} ; p=.10$ ).

Two ANCOVA analyses controlling for age and average daily step counts were performed to further assess the relationship between AST. BMI and waist circumference. The results are summarized in Table 2. In both ANCOVA models, the relationships did not differ according to gender. In model 1, the relationship between AST and BMI fell at the significance threshold ( $\mathrm{F}=3.75 ; p=05)$. The estimated marginal means for BMI were $18.6 \mathrm{~kg} / \mathrm{m}^{2}(95 \% \mathrm{CI}=17.5-19.8)$ for the active commuters and $19.9 \mathrm{~kg} / \mathrm{m}^{2}(95 \% \mathrm{CI}=19.3-20.5)$ for the passive commuters. Step counts were associated with BMI, but not age (respectively $\mathrm{F}=4.27 ; p=.04$ and $\mathrm{F}=2.41: p=12$ ). According to the second ANCOVA model, the active commuters had lower mean waist circumferences, but the difference was not significant $(\mathrm{F}=2.79: p=.10)$. The estimated marginal means for waist circumference were 
Table 2 Results of the ANCOVA Analyses for Body Mass Index and Waist Circumference

\begin{tabular}{lcccc}
\hline Source & \multicolumn{2}{c}{ Body mass index } & \multicolumn{2}{c}{ Waist circumference } \\
\cline { 2 - 5 } & F value & $p$ value & F value & $p$ value \\
\hline Corrected model & 2.08 & 0.07 & 3.41 & 0.01 \\
Steps per day & 4.27 & 0.04 & 8.24 & 0.01 \\
Age & 2.41 & 0.12 & 5.44 & 0.02 \\
Mode of transportation & 3.75 & 0.05 & 2.79 & 0.10 \\
Gender & 0.01 & 0.94 & 0.03 & 0.86 \\
Transport * Gender & 0.04 & 0.84 & 0.28 & 0.60 \\
\hline
\end{tabular}

N.B. For BMI, the $R^{2}=.05$ (Adjusted $R^{2}=026$ ) and for waist circumference, $R^{2}=, 092$ (Adjusted $R^{2}=,(065)$

$69.0 \mathrm{~cm}(95 \% \mathrm{Cl}=65.2-72.8)$ and $72.6 \mathrm{~cm}(95 \% \mathrm{Cl}=70.7-74.4)$ for the active and passive commuters respectively. The second model also revealed that both step counts and age were significantly associated with waist circumference (respectively $\mathrm{F}=8.24 ; p<.01$ and $\mathrm{F}=5.44 ; p=.02$ ).

\section{Discussion}

The purpose of our investigation was to assess the relationship between AST. physical activity levels and body composition. The most important finding was that BMI was significantly lower among the active commuters, when compared with the passive commuters (a mean difference of $1.2 \mathrm{~kg} / \mathrm{m}^{2}$ ), even when controlling for age and daily step counts. The combined prevalence of overweight and obesity was approximately $46 \%$ lower among the active commuters. Moreover, for a 10 year old child, the mean BMI observed in the passive commuters $\left(19.9 \mathrm{~kg} / \mathrm{m}^{2}\right)$ correspond to the threshold for overweight according to the IOTF classification (11). These differences are clinically relevant given the wide range of medical and psychosocial complications associated with childhood obesity $(21,28)$.

It is of great concern that a decline in the prevalence of AST has been observed among Canadian (8). American (29) and Australian (42) children and adolescents during the last decades. These trends occurred in parallel with rapid increases of childhood obesity. For example, Tremblay and colleagues (39) compared the results of the children and adolescents who participated in the 1981 Canada Fitness Survey and the 2007-2009 Canadian Health Measure Survey. They found that the later had a $1.1 \mathrm{~kg} / \mathrm{m}^{2}$ higher BMI, and the combined prevalence of overweight and obesity doubled (39). However, the potential contribution of the reduction in the prevalence of AST on the increase of body weight has not been evaluated empirically.

Our results are in contrast with the findings of a systematic review on the topic (20), where most studies did not report a relationship between AST and BMI, but the authors noted that the existing evidence was too weak to draw a strong conclusion. It was also argued that the absence of significant differences could result from 
rather short distances between home and school among most active commuters (20). More recently, German researchers observed an inverse association between AST and obesity when objectively measured distance to school was taken into account, suggesting a dose-response relationship (26). Similarly, prospective data from the Québec Longitudinal Study of Child Development found that sustained AST over two years in young children predicted lower BMI values (31).

The current study reveals a trend toward smaller waist circumferences among the active commuters. As reflected by the large standard deviation, it can be hypothesized that the variability between participants precluded the observation of a significant difference. To our knowledge, earlier publications did not assess the association between AST and waist circumference. This is surprising given the strength of the association between waist circumference and health risks such as insulin resistance, elevated cholesterol and blood pressure in youth $(44,45)$. Some pedometry-based studies have shown that children who did not meet the step recommendations were more likely to have an elevated waist circumference or a higher body fat percentage (17,19). Moreover, Eisenmann and colleagues' (19) demonstrated a dose-response relationship between step counts categories, $\mathrm{BMI}$ and waist circumference.

The current findings highlight the need for further investigation on the impact of AST on body composition variables. To date, most published studies have: 1) used a cross-sectional methodology $(20) ; 2$ ) relied only on BMI as an indicator of obesity, which may not accurately reflect body composition (44): 3) did not distinguish between modes of AST such as walking and cycling (7); and 4) did not take into account the volume of AST $(20,31)$. In addition, experimental trials (or quasi-experimental studies with a control group) would be needed to establish the direction of the relationship between both variables.

With respect to physical activity levels, the active commuters accumulated 662 more steps/day, but the difference was not significant. In contrast, other researchers who examined the impact of AST on daily step counts have found larger differences between groups (1,18.23). In addition, most studies in which physical activity was measured with accelerometry have shown that active commuters were significantly more active on a daily basis ( 16,20 ), especially during the hour preceding and following the school day (13).

The main limitation of the current study is the relatively small sample size, which may explain the lack of significant differences between groups for waist circumference and average daily step counts. With respect to step counts, there could be a discrepancy between the mode of transport reported by the participants and the mode they used during the monitoring period. In addition, the number of active commuters was too low to assess the impact of walking and cycling separately. Despite the inclusion of schools in urban and rural areas and with various socioeconomic levels, the sample may not be representative of the population, which limits the generalizability of the findings. In addition, given the cross-sectional design, it is not possible to confirm that children were leaner because they engaged in AST. All data were collected in the fall and early winter, and transportation behaviors may be subject to seasonal variations like overall physical activity level (10).

It seems unlikely that participant reactivity had a major impact on the results. The average step count values were relatively low (II 924 and II 262 for the active and passive commuters respectively), but similar to those obtained in the 
CANPLAY study with a population sample of 19789 children and adolescents aged 5-19 (12 259 for boys and 10906 for girls; 15) and to other North American studies, as reported in a recent systematic review (6). Furthermore, there is no reason to believe that potential participant reactivity would be different among active or passive commuter groups. Finally, researchers who compared step counts obtained from sealed vs. unsealed pedometers reported no evidence of a reactivity bias $(30,33,41)$.

The strengths of this study include the objective measurement of physical activity which, unlike questionnaires, is not subject to recall bias (3); b) the utilization of previously validated thresholds for the step count data; $c$ ) the measurement of both BMI and waist circumference and d) the control for daily step counts in the assessment of the relationship between AST and body composition variables.

\section{Conclusion}

In the current study, the children who reported using active modes of transportation had lower BMI values, and were less likely to be overweight and obese. When controlling for age and daily step counts, this relationship remains significant. This difference is clinically relevant given the wide range of complications associated to childhood obesity. Lack of statistical power and high variability between participants may have prevented the observation of significant differences for outcome variables such as waist circumference and physical activity level. In conclusion. these findings emphasize the need for further research on the outcomes of AST. as well as interventions to promote AST.

\section{Acknowledgments}

This component of the CAPL study was funded by the Canadian Institutes of Health Research, The Children's Hospital of Eastern Ontario Research Institute, The Public Health Agency of Canada, the Ontario Ministry of Health Promotion, and ParticipACTION. The main author holds a Frederick Banting and Charles Best Canada Graduate Scholarship from the Canadian Institutes of Health Research (CIHR).

\section{References}

1. Abbott, R.A., B. Macdonald, S. Nambiar, and P.S.W. Davies. The association between walking to school, daily step counts and meeting step targets in 5- to 17-year-old Australian children. Pediatr, Exerc. Sci. 21:520-532, 2009.

2. Active Healthy Kids Canada. Healthy habits start earlier than you think. The Active Healthy Kids Canada report card on physical activity for children and youth. Toronto, On, 2010.

3. Adamo, K., S.A. Prince, A.C. Tricco, S. Connor-Gorber, and M.S, Tremblay. A comparison of indirect versus direct measures for assessing physical activity in the pediatric population: a systematic review, Int. J. Pediatr, Obes, 4:2-27, 2008.

4. Andersen, L.B.. M. Harro, L.B. Sardinha, et al. Physical activity and clustered cardiovascular risk in children: a cross-sectional study (The European Youth Heart Study). Lancet. 368:299-304, 2006.

5. Bauman, A., M. Allman-Farinelli. R. Huxley, and W.P.T. James. Leisure-time physical activity alone may not be a sufficient public health approach to prevent obesity - A focus on China. Obes. Rev, 9(Suppl, 1):119-126, 2008. 
6. Beets, M.W., D. Bonstein, A. Beighle, B.J. Cardinal, and C.F. Morgan. Pedometermeasured physical activity pattems in youth: a 13-country review, Am. J. Prev. Med. $38(2): 208-216,2010$.

7. Bere, E., and L.B. Andersen. Why no support for an association between active commuting to school and weight status in the literature. J Phys Act Health. 6:533-534. 2006 .

8. Buliung, R.N., R. Mitra, and G. Faulkner. Active school transportation in the Greater Toronto Area, Canada: An exploration of trends in space and time (1986-2006). Prev, Med. 48:507-512, 2009.

9. Canadian Society for Exercise Physiology. The Canadian Physical Activity, Fitness \& Lifestyle Approach, Ottawa, On, 2003.

10. Carson, V., and J.C. Spence. Seasonal variation in physical activity among children and adolescents: a review. Pediatr. Exerc. Sci. 22:81-92, 2010,

11. Cole, TJ., M.C. Bellizzi. K.M. Flegal, and W.H. Dietz. Establishing a standard definition for child overweight and obesity wotldwide: international survey. BMJ. 300(7244):1240, 2000.

12. Colley, R., S. Connor Gorber, and M.S. Tremblay. Quality control and data reduction procedures for accelerometry-derived measures of physical activity. Health Rep. $21(1): 1-8,2010$.

13. Cooper. A.R.,A.S. Page, L.J. Foster, and D. Qahwaji. Commuting to school: are children who walk more active. Am. J. Prev. Med. 25(4):273-276, 2003 ,

14. Craig, C.L., C. Tudor-Locke, S. Cragg, and C. Cameron. Process and treatment of pedometer data collection for youth: The Canadian Physical Activity Levels Among Youth study. Med. Sci. Sports Exerc: 42(3);430-435, 2010.

15. Craig, C.L..C.Cimeron, J.M. Griffiths, and C. Tudor-Locke, Descriptive epidemiology of youth pedometer-determined physical activity: CANPLAY.Med. Sci. Sports Exere. 42(9):1639-1643, 2010.

16. Davison. K.K., J.L. Werder, and C.T. Lawson. Children's active commuting to school: current knowledge and future directions. Prev. Chronic Dis. 5(3):1-11, 2008.

17. Duncan, J.S, G, Schofield, and E.K. Duncan. Pedometer-determined physical activity and body composition in New Zealand children. Med. Sci. Sports Exerc. 38(8):1402$1409,2006$.

18. Duncan, E.K., J.S. Duncan, and G. Schofield. Pedometer-determined physical activity and active transport in girls. Int. J. Behav. Nutr. Phys. Act. S(1): 2008. http://ijbnpa. org/content $5 / 1 / 2$.

19. Eisenmann, J.C., K.R. Laurson, E.E. Wickel, D. Gentile, and D. Walsh. Utility of pedometer step recommendations for predicting overweight in children. Int. J. Obes. $31: 1179-1182,2007$.

20. Faulkner, G.E.J., R.N. Buliung. P.K. Flora, and C. Fusco. Active school transport, physical activity levels and body weight of children and youth: a systematic review. Prev: Med. 49:3-8, 2009.

21. Han, J.C., D.A. Lawlor. and S.Y.S. Kimm. Childhood obesity. Lancet. 375(9527):1737$1748,2010$.

22. Health Canada. Canada's Physical Activity Guide for Children. Ottawa, ON: Health Canada: 2002. Publication H39-61 1/2002-2E

23. Hohepa, M., G. Schofield, G.S. Kolt, R. Scragg, and N. Garrett. Pedometer-determined physical activity levels of adolescents: differences by age, sex, time of week, and transportation mode to school. I Phys Act Health. 5(Suppl. 1):140-152, 2008.

24. Jago, R., N. Wedderkopp, P.L. Kristensen, et al. Six-year change in youth physical activity and effect on fasting insulin and HOM.A-IR. Am. J. Prev. Med. 35(6):554-560, 2008.

25. Janssen, I., M. Shields, C.L. Craig, and M.S. Tremblay. Prevalence and secular changes in abdominal obesity in Canadian adolescents and adults, 1981 to 2007-2009. Obes. Rev, . 
26. Landsberg, B., S. Plachta-Danielzik, D. Much, M. Johannsen, D. Lange, and M.J. Müller. Associations between active commuting to school, fat mass and lifestyle factors in adolescents: the Kiel Obesity Prevention Study (KOPS). Eur. J. Clin. Nutr. $62: 739-747,2008$.

27. Lloyd, M., R.C. Colley, and M.S. Tremblay.Advancing the debate on 'fitness testing' for children: perhaps we're riding the wrong animal. Pediatr. Exerc. Sci. 22:176-182. 2010.

28. Lobstein, T., L. Baur, and R. Uauy. Obesity in children and young people: a crisis in public health. Obes. Rev. 5(Suppl. 1):4-85, 2004.

29. McDonald, N.C. Active transportation to school: Trends among U.S. schoolchildren. 1969-2001. Am. J. Prev. Med. 32(6):509-516, 2007.

30. Ozdoba, R., C. Corbin, and G. Le Masurier. Does reactivity exist in children when measuring activity levels with unsealed pedometers? Pediatr. Exerc. Sci. 16:158-166. 2004.

31. Pabayo, R., L. Gauvin, T.A. Barneti, B. Nikiéma, and L. Séguin. Sustained active transportation is associated with a favorable body mass index trajectory across the early school years: Findings from the Québec Longitudinal Study of Child Development birth cohort. Prev. Med. 50(Suppl. 1):S59-S64, 2010.

32. Rowe, D., M. Mahar, T.D. Raedeke, and J. Lore. Measuring physical activity in children with pedometers: reliability, reactivity, and replacement of missing data. Pediatr. Exerc. Sci. 16(4):343-354, 2004.

33. Rowlands, A.V., and R.G. Eston. The measurement and interpretation of children's physical activity. J Sports Sci Med. 6:270-276, 2007.

34. Sallis, J.F., R.B. Cervero, W. Ascher, K.A. Hendersen, M.K. Kraft, and J. Kerr. An ecological approach to creating active living communities. Annu. Rev. Public Health. $27: 297-322,2006$.

35. Shephard, R.J. Is active commuting the answer to population health? Sports Med. 38(9):75I-758, 2008 .

36. Strong, W.B., R.M. Matina, C.J. Blimkie, et al. Evidence based physical activity for school-age youth. J. Pediatr. 146:732-737, 2005.

37. Tremblay, M.S., D.W. Esliger, A. Tremblay, and R. Colley, Incidental movement, lifestyle-embedded physical activity and sleep: new frontiers in physical activity assessment. Can. J. Public Health. 98(Suppl. 2):S208-S216, 2007.

38. Tremblay, M.S., and M. Lloyd. Physical literacy measurement - the missing piece. Physical and Health Education Canada Journal. 76(1):26-30, 2010.

39. Tremblay, M.S., M. Shields, M. Laviolette, C.L. Craig. I. Janssen, and S. Connor Gorber. Fitness of Canadian children and youth: Results from the 2007-2009 Canadian Health Measures Survey. Health Rep. 21(1):1-14, 2010.

40, Tudor-Locke, C., L. Burkett, J.P. Reis, B.E. Ainsworth, C.A. Macera, and D.K. Wilson. How many days of pedometer monitoring predict weekly physical activity in adults? Prev. Med. 40א3):293-298, 2005.

41. Tudor-Locke, C., J.T. Mcclain, T.L. Hart, S.B. Sisson, and T.L. Washington. Pedometry methods for assessing free-living youth. Res. Q. Exerc. Sport. 80(2):175-184, 2009.

42. van der Ploeg, H.P., D. Merom, G. Corpuz, and A.E. Bauman. Trends in Australian children travelling to school 1971-2003: Burning petrol or carbohydrates? Prev. Med. $46: 60-62,2008$.

43. Wang, $Y_{n}$, and T. Lobstein, Worldwide trends in childhood overweight and obesity, Int. J. Pediatr. Obes. 1:11-25, 2006.

44. Wells, J.C.K., and M.S. Fewtrell. Measuring body composition. Arch. Dis. Child. 91:612-617,2006.

45. Zimmet, P., K.G.M.M. Alberti, F. Kaufman, et al. The metabolic syndrome in children and adolescents - an IDF consensus report. Pediatr. Diabetes. 8:299-306, 2007. 
Chapter 4. Study III: "Relationships among active transportation, physical activity, fitness and cardiovascular disease risk factors: findings from the Canadian Health Measures Survey"

Authors: Richard Larouche, Guy Faulkner, Michelle Fortier, Mark S. Tremblay

Status: Submitted for publication in American Journal of Preventive Medicine on May 27, 2013.

Submitted for presentation at the 2013 Canadian Society for Exercise Physiology Conference. 
Relationships among active transportation, physical activity, fitness and cardiovascular disease risk factors in adolescents: the Canadian Health Measures Survey

Richard Larouche, M.Sc. ${ }^{1,2}$, Guy Faulkner, Ph.D. ${ }^{3}$, Michelle Fortier, Ph.D. ${ }^{2}$, Mark S. Tremblay, Ph.D. ${ }^{1,2,4}$ 
Abstract word count: 249

Word count: 2,965

Number of pages: 34

Number of tables: 5

\section{Conflict of interest statement:}

Richard Larouche was supported by a Frederick Banting and Charles Best Doctoral Award from the Canadian Institutes of Health Research and holds an Excellence Scholarship from the University of Ottawa.

Guy Faulkner: no conflicts of interest.

Michelle Fortier: no conflicts of interest.

Mark S. Tremblay worked for Statistics Canada in the development of the Canadian Health Measures Survey; he is the Chief Scientific Officer of Active Healthy Kids Canada but receives no payment for this role; he has no commercial associations relevant to this paper or analysis.

Publishable statement: the authors have no conflicts of interest to disclose. 


\begin{abstract}
:
Background: Active transportation (AT; e.g. the use of non-motorized travel modes like walking and cycling) is increasingly promoted as a way to increase physical activity (PA) levels in youth. To date, most of the research has focused solely on the trip to/from school, and associations of AT with cardiovascular risk factors have seldom been examined in adolescents.
\end{abstract}

Purpose: The present study aimed to address these important research gaps using data from the nationally-representative 2007-2009 Canadian Health Measures Survey.

Methods: Participants ( $\mathrm{N}=1,016$ adolescents aged 12-19 years) reported the weekly time they spent walking and cycling for utilitarian purposes, and were instructed to wear an Actical accelerometer on the right hip for 7 consecutive days. They underwent a series of physical tests (including measures of fitness, body composition and blood pressure) following standardized protocols. Blood samples were drawn by a phlebotomist. In 2013, differences in PA and healthrelated outcomes across levels of walking and cycling were assessed with ANCOVA analyses adjusted for age, gender and parental education level.

Results: Both reported walking and cycling were significantly associated with daily minutes of moderate-to-vigorous PA, but not with light PA or sedentary behavior. Adolescents who engaged in utilitarian cycling had significantly lower cholesterol levels and a lower total cholesterol/HDL ratio. No differences were found for the other outcomes. 
Conclusion: Active travel is associated with higher daily PA levels. Further efforts to design, implement and evaluate interventions to promote AT in youth are warranted. Cycling may be associated with greater health benefits than walking in youth. 


\section{Introduction}

Physical activity (PA) guidelines recommend that children and youth engage in at least 60 minutes of daily moderate- to vigorous-intensity physical activity (MVPA). ${ }^{1,2}$ However, compliance with this guideline is very low according to recent population-based studies. ${ }^{3-6}$ For example, accelerometry data from the 2007-2009 Canadian Health Measures Survey (CHMS) indicated that only $7 \%$ of Canadian children and youth met the PA guidelines, and this proportion was lower among adolescents. $^{4}$

Insufficient PA among children and youth is linked to many deleterious health outcomes including poorer aerobic fitness ${ }^{7}$ and mental health ${ }^{8}$, lower glucose tolerance and higher fasting insulin levels ${ }^{9}$, and even cardiovascular disease (CVD) risk factors. ${ }^{10,11}$ It has also been found that CVD risk factors tend to track from adolescence to adulthood. ${ }^{12}$ As a strategy to address this major public health issue, health authorities are increasingly promoting active transportation (AT), that is the use of non-motorized travel modes such as walking and cycling to travel to and from various destinations including schools, workplaces, parks and shops. ${ }^{13,14}$

Among children and youth, current evidence suggests that AT is associated with higher overall PA levels and that individuals who cycle to/from school have greater aerobic fitness than those using motorized travel modes. ${ }^{15,16}$ However, several knowledge gaps remain to be addressed. First, most AT studies have focused solely on the trip to/from school, thereby ignoring active trips to other destinations. Consequently, it is unclear how much AT in general contributes to overall PA levels. Second, due to small sample sizes and/or low numbers of cyclists, several 
researchers have combined AT modes in their analyses, despite differences in PA intensity

between walking and cycling. ${ }^{17}$ Third, only three studies have evaluated the relationship between AT and CVD risk factors, and their findings have been inconsistent. ${ }^{18-20}$

Thus, the present study aimed to address these important limitations using data from adolescents who participated in the nationally-representative 2007-2009 CHMS. Specifically, differences in accelerometry-measured PA, indicators of body composition, physical fitness and CVD risk factors across levels of walking and cycling were investigated.

\section{Methods}

Participants. The 2007-09 CHMS collected nationally-representative data from 5,604

Canadians aged 6-79 years living in private households at the time of the survey. ${ }^{21-24}$ Approximately $96 \%$ of Canadians are represented; residents of Reserves, Crown lands, institutions, some remote regions and full-time Canadian Forces employees are excluded. Data were collected from March 2007 to February 2009 in 15 sites across Canada. Ethical approval was obtained from Health Canada's Research Ethics Board. ${ }^{22}$ The survey included a home interview and a visit to a mobile examination center for a series of physical measurements. ${ }^{21}$ Written informed consent was obtained from participants aged 14 and older. For younger children, a parent (or guardian) provided written consent in addition to written assent from the child. 
Of the selected households, $69.6 \%$ agreed to participate. Among the recruited households, $88.5 \%$ of 6-19 years olds completed the questionnaire from whom $86.9 \%$ participated in the mobile examination center component. Hence, the overall response rate was 53.5\%. For the present analyses, data from 12-19 years old adolescents were used ( $\mathrm{N}=1,016,48.8 \%$ female). Younger children were not questioned on their AT.

Procedures. A detailed description of data collection procedures, screening guidelines and eligibility criteria for the different measurements is provided in the CHMS Data User's Guide which is available online. ${ }^{25} \mathrm{AT}$ was assessed with 2 items (one for walking and one for cycling), as follows: "in a typical week in the past 3 months, how many hours did you usually spend walking [or bicycling] to work or to school or while doing errands?" Response options were: 1) none; 2) < 1 hour; 3 ) 1 to 5 hours; 4) 6 to 10 hours; 5) 11 to 20 hours; 6) $\geq 20$ hours. For the present analyses, 3 categories were used for walking ( $<1$ hour, 1 to 5 hours, $>5$ hours) and cycling (none, $<1$ hour, $\geq 1$ hours) based on the observed frequency distributions.

After completion of the mobile examination centre visit, participants were instructed to wear an Actical accelerometer over their right hip on an elasticized belt during waking hours for 7 consecutive days, except during water-based activities (e.g. bathing and swimming). This accelerometer has been validated for measuring PA in children and youth. ${ }^{26-27}$ Data were collected in 60-second epochs. The protocols used to assess body composition and physical fitness were taken from the Canadian Physical Activity, Fitness and Lifestyle Approach ${ }^{28}$ and described in more details elsewhere. ${ }^{29}$ These tests were conducted by trained specialists with certification from the Canadian Society for Exercise Physiology. ${ }^{29}$ Cardiovascular fitness 
$\left(\mathrm{VO}_{2} \mathrm{max}\right)$ was estimated with the modified Canadian Aerobic Fitness Test (mCAFT). Grip strength was assessed with a dynamometer and trunk flexibility was assessed with the sit-andreach test. BMI $\left(\mathrm{kg} / \mathrm{m}^{2}\right)$ was computed from height and weight measured to the nearest $0.1 \mathrm{~cm}$ and $0.1 \mathrm{~kg}$ respectively. Waist circumference was measured at the end of a normal expiration to the nearest $0.1 \mathrm{~cm}$ at the mid-point between the last rib and the top of the iliac crest.

Blood pressure was measured with an automated monitor. ${ }^{30}$ Six measurements were taken at 1min intervals and average systolic and diastolic blood pressure was calculated from the last 5 measurements. In the CHMS, fasted blood samples were only obtained from participants who visited the mobile examination centre in the morning. Therefore, non-fasted blood markers were used in the present analyses (HDL cholesterol, total cholesterol, total cholesterol/HDL ratio, blood glucose and glycohemoglobin [Hbalc]). Blood samples were taken by a certified phlebotomist and analyzed at the Health Canada Laboratory following standardized protocols. ${ }^{25}$

Data treatment. Accelerometry data were treated as detailed by Colley et al. ${ }^{4,31}$ Briefly, a valid day was defined as $\geq 10$ hours of wear time, and participants with $\geq 4$ valid days were included in the accelerometry subsample $(\mathrm{n}=729)$. Daily wear time was obtained by subtracting non-wear time (defined as periods of $\geq 60$ minutes of consecutive zero counts) from 24 hours. The cutpoints used for determining PA intensity were: $<100$ counts/min = sedentary behavior; 100-1499 counts $/ \mathrm{min}=$ light $\mathrm{PA} ; \geq 1500-6499 \mathrm{counts} / \mathrm{min}=\operatorname{moderate} \mathrm{PA} ; \geq 6500 \mathrm{counts} / \mathrm{min}=$ vigorous PA. The majority of participants did not engage in any vigorous PA. Hence, moderate and vigorous PA were combined as MVPA and log-transformed given the skewness of the distribution. The z-scores for waist circumference, systolic blood pressure, HDL cholesterol 
(reverse coded), total cholesterol and blood glucose were added to obtain a clustered cardiovascular risk score following an approach similar to that of Ekelund et al. ${ }^{32}$

Statistical analyses. Analyses were performed in 2013 with Stata version 12 using survey weights, and alpha was set at .05. To account for the complex survey design of the CHMS, 95\% confidence intervals were estimated using the bootstrap technique. ${ }^{25,33,34}$ Chi-squared analyses assessed differences in levels of walking and cycling between genders, age groups (dichotomized as $\leq 15$ years vs. $\geq 16$ years) and levels of parental education (categorized as university, college, and less than college). These variables have previously been found to be important correlates of AT and/or PA. ${ }^{35-38}$ Gender differences in outcome variables were evaluated with t-tests (or chisquare tests when appropriate). A series of analyses were used to assess the association of utilitarian walking and cycling with measures of PA, body composition, physical fitness and CVD risk factors. First, univariate associations were examined with one-way ANOVAs (model 1). Second, a series of ANCOVAs adjusted for participant's age (entered as a continuous variable), gender and the highest parental education level were run (model 2). Separate models were done for walking and cycling using the generalized linear model procedure. Within each series of models, Holm's method was used to adjust the $p$ values for multiple comparisons. ${ }^{39}$

\section{Results}

Descriptive characteristics of the sample and gender differences are shown in Table 1. Of note, about half of the participants reported walking between 1 and 5 hours per week and an additional $22 \%$ reported walking for more than 5 hours per week. In contrast, $80.7 \%$ of participants 
reported no cycling at all during the last 3 months, with the remainder rather evenly distributed within the under 1 hour and over 1 hour categories.

Table 2 and 3 illustrate the associations of demographic variables with levels of walking and cycling respectively. Levels of walking did not differ according to gender but large differences were found for cycling $(\mathrm{F}=15.41 ; p<0.001)$. In addition, about twice as many $16-19$ years old adolescents engaged in at least 5 hours of utilitarian walking per week compared to their 12-15 years old counterparts $(\mathrm{F}=9.94 ; p=0.001)$. However, older adolescents were less likely to cycle $(\mathrm{F}=4.24 ; p=0.028)$. Parental education was not associated with levels of walking or cycling.

Associations of levels of walking with PA and health-related outcomes are shown in Table 4. In univariate models, a significant association was found between utilitarian walking and higher waist circumferences $(\mathrm{t}=2.45 ; p=0.032)$; this difference was no longer significant in the adjusted models. Similar trends were noted for the other body composition indicators, but the differences were not statistically significant. In the adjusted models, time spent walking was associated with MVPA ( $\mathrm{t}=3.19 ; p=0.009)$, but no dose-response gradient was evident. No differences were found for other outcomes.

Table 5 shows the associations of levels of cycling with PA and health-related outcomes. In univariate models, adolescents who reported more utilitarian cycling had significantly higher cardiovascular fitness $(\mathrm{t}=8.08, p<0.001)$, daily MVPA $(\mathrm{t}=4.29 ; p=0.001)$, and had lower total cholesterol ( $\mathrm{t}=-3.58 ; p=0.004)$. In adjusted models, the observed differences in MVPA ( $\mathrm{t}=3.29$; $p=0.007)$ and total cholesterol $(\mathrm{t}=-3.29 ; p=0.007)$ remained significant and cycling was also 
associated with lower cholesterol/HDL ratios $(\mathrm{t}=-3.13 ; p=0.010)$. The association with cardiovascular fitness was no longer significant after adjusting for gender.

\section{Discussion}

The present study is the first to examine the associations of AT (not restricted to the trip to/from school) with measures of PA, physical fitness, body composition and CVD risk factors in a nationally-representative sample of adolescents. Interestingly, the proportion of 16-19 years old adolescents reporting walking at least 5 hours per week was twice as high as that of younger adolescents. This finding differs from previous North American investigations focusing only on AT to/from school, which have generally found that older adolescents were less likely to walk or cycle, ${ }^{36,40,41}$ suggesting that older adolescents may do more AT trips to non-school destinations.

Consistent with literature reviews of AT to/from school ${ }^{15,42}$, both walking and cycling were significantly associated with accelerometry-measured MVPA in the adjusted models. However, no associations between AT and sedentary behavior were observed. This may be attributable to the very large amount of sedentary time in Canadian children and youth ( $\sim 62 \%$ of waking hours $)^{4}$. Relationships between AT and health-related outcomes differed according to travel mode and are therefore discussed separately.

Associations between utilitarian walking and health-related outcomes. Despite the observed difference in MVPA, there were no significant associations between reported walking and health-related outcomes in the adjusted models. There is currently mixed evidence regarding the 
association of walking to/from school with cardiovascular fitness and indicators of body composition. ${ }^{15,16}$ To date, three other studies have assessed relationships between walking to/from school and CVD risk factors in smaller samples. ${ }^{18-20}$ A cross-sectional study noted that Portuguese children who walked to/from school were less likely to have a waist circumference greater than the $90^{\text {th }}$ percentile and HDL-cholesterol below $40 \mathrm{mg} / \mathrm{dl} .{ }^{20}$ In contrast, two prospective studies reported no significant differences in health-related outcomes between children who walked to school and those who were driven. ${ }^{18,19}$

Shephard ${ }^{17}$ has argued that in children and youth, the PA intensity of utilitarian walking may be insufficient to improve cardiovascular fitness and other health outcomes. While the available evidence provides some support for this hypothesis, the lack of associations may also reflect statistical confounding. Although the present analyses were adjusted for parental education, it was not possible to adjust for other socioeconomic characteristics such as household income (asked only to older participants in the CHMS) or car ownership (not assessed). North American studies have consistently found that walking to school is more common in children from lower SES households. ${ }^{36,41,43}$ Other studies have found significant associations between low SES and overweight/obesity in children and youth. ${ }^{44,45}$

Associations between utilitarian cycling and health-related outcomes. Of particular interest, almost four times more boys than girls engaged in utilitarian cycling for at least one hour per week. Similarly, using data from six waves of the US National Household Travel Survey, McDonald $^{35}$ found that cycling to school rates were 2-3 times higher in boys than girls. Transport surveys indicated that men do about $70-75 \%$ of cycling trips in countries such as 
Australia, Canada, the UK and the US. ${ }^{46}$ Interestingly, there are no gender differences in countries such as The Netherlands, Denmark and Germany where cycling infrastructure is more extensive and injury rates are markedly lower. ${ }^{46}$

In univariate analyses, utilitarian cycling was associated with greater aerobic fitness, and the magnitude of this difference ( $\sim 6-10 \%$; Table 5$)$ was similar to studies of cycling to/from school. ${ }^{15}$ However, this association was no longer significant after controlling for gender. Nevertheless, longitudinal studies have shown that switching from an inactive travel mode to cycling for the trip to/from school can lead to an increase in aerobic fitness. ${ }^{19,47}$ For example, Danish children who started cycling during a 6-year follow-up period achieved $9 \%$ greater aerobic fitness at 15 years of age than those who did not cycle at baseline and follow-up. ${ }^{47}$ Moreover, in a 12-week intervention, youth who started cycling to/from school achieved a 7.9\% mean increase in $\mathrm{VO}_{2}$ peak. ${ }^{48}$

In the present study, cyclists tended to have more favorable body composition indicators, but differences were not statistically significant. Systematic reviews have found mixed evidence for such associations. ${ }^{15-16}$ In addition, cycling was not associated with measures of trunk flexibility and grip strength. Only one other study has assessed the relationship between AT and other indicators of physical fitness in adolescents. ${ }^{49}$ Danish adolescents who cycled to school were more flexible and had greater abdominal endurance, and the authors hypothesized that this may be due to the use of road bikes. However, no differences were reported for measures of muscular strength. 
Adolescents who engaged in utilitarian cycling had lower total cholesterol and total cholesterol/HDL ratio even after adjustment for age, gender and parental education, although no dose-response relationship was evident. These findings are somewhat consistent with those of the Danish arm of the European Youth Heart Study. ${ }^{18}$ Furthermore, Andersen and colleagues ${ }^{18}$ observed that children who started to cycle during the 6-year follow-up period had a better lipid profile and a lower clustered CVD risk score compared to those who did not cycle at any time point. However, Chillón et al. ${ }^{19}$ reported no association between cycling to school and CVD risk factors (except cardiovascular fitness) in a sample of 262 adolescents among the original 907 participants in the Swedish arm of the European Youth Heart Study.

In comparison, prospective cohort studies of adults have found that AT to work is associated with reduced risks of $\mathrm{CVD}^{50-52}$ and all-cause mortality ${ }^{53,54}$. Recent studies have also found that habits are a strong determinant of travel modes among both children ${ }^{55}$ and adults ${ }^{56-57}$. Therefore, developing the habit to engage in AT during childhood and adolescence may be important even though health benefits may not be immediate.

Limitations and strengths. The main limitation of this study is the cross-sectional design which makes it impossible to determine the direction of observed relationships. Second, despite adjustment for parental education, low SES may remain a confounder of the relationship between AT and health-related outcomes. Third, statistical adjustment was limited by the survey design allowing only 11 degrees of freedom. However, having a small number of degrees of freedom also makes the analyses more conservative. ${ }^{58}$ Fourth, analyses were not adjusted for MVPA because AT represents an important source of MVPA. ${ }^{15}$ For example, school journeys accounted 
for about a third of daily MVPA in British secondary school children. ${ }^{59}$ To address this issue, future studies should consider using a combination of accelerometers and GPS to subtract the MVPA accumulated through AT from the total time spent in MVPA. Then, statistical analyses could control for "non-AT" MVPA. Fifth, fasted blood samples were only obtained from a subsample of participants; this limited the choice of blood markers to examine. Sixth, although hip-worn accelerometers are valid and reliable for measuring total PA in adolescents, they underestimate the intensity of activities such as cycling ${ }^{60}$, so differences in MVPA between categories of utilitarian cycling may be larger than reported here. Seventh, although the current study considered utilitarian trips to a broader range of destinations, active trips to the park or to visit family/friends were not specifically examined. Finally, because data were collected throughout the year, it is possible that adolescents who cycle during warmer months were categorized as non-cyclists because they did not cycle in the 3 months prior to the interview. While rates of cycling to school have been shown to decrease during the winter in Norway ${ }^{61}$, no seasonal variations in walking were found in the Greater Toronto Area. ${ }^{62}$

The large nationally-representative sample of adolescents is an important strength of the study. A second strength is that all outcome variables were directly measured. This avoided social desirability and recall biases that are common when using self-reports of PA and body composition indicators. ${ }^{63,64}$ Third, walking and cycling were examined separately, as recommended previously. ${ }^{17}$ Fourth, data collection occurred throughout the year; minimizing the possibility that population estimates of PA are affected by seasonal variations. ${ }^{65}$ Fifth, this is the first study to assess the relationship between AT in general (e.g. not just to/from school) and 
measures of CVD risk factors in adolescents. Finally, a large number of variables that may be influenced by adolescents' travel patterns were examined.

\section{Conclusion}

Consistent with studies of AT to/from schoo ${ }^{15}$, the present study found that Canadian adolescents who reported more time engaging in AT accumulated larger amounts of objectivelymeasured daily MVPA. Thus, future efforts to design, implement and evaluate interventions aiming to promote $\mathrm{AT}$ in children and youth are warranted. Furthermore, engagement in utilitarian cycling was associated with lower cholesterol levels and a lower total cholesterol/HDL ratio.

\section{Acknowledgements}

The authors would like to acknowledge Dr. Rachel Colley for her help in processing accelerometer data and her contribution to the design of the analyses. RL holds an Excellence Scholarship from the University of Ottawa. Although the present research and analyses are based on data from Statistics Canada, the views expressed do not represent those of Statistics Canada.

\section{References}

1. Tremblay MS, Warburton DER, Janssen I, et al. New Canadian physical activity guidelines. Appl Physiol Nutr Metabol. 2011;36:36-46. 
2. United States Department of Health and Human Services. Physical Activity Guidelines Advisory Committee Report, 2008. Washington, DC. 2008.

3. Basterfield L, Adamson AJ, Pearce MS, Reilly JJ. Stability of habitual physical activity and sedentary behavior monitoring by accelerometry in 6- to 8-year-olds. J Phys Act Health. 2011;8:543-7.

4. Colley RC, Garriguet D, Janssen I, Craig CL, Clarke J, Tremblay MS. Physical activity of Canadian children and youth: Accelerometer results from the 2007 to 2009 Canadian Health Measures Survey. Health Reps. 2011;22(1):15-23.

5. Troiano R, Berrigan D, Dodd KW, Mâsse LC, Tilert T, McDowell M. Physical activity in the United States measured by accelerometer. Med Sci Sports Exerc. 2008;40:181-8.

6. Hallal P, Andersen LB, Bull FC, et al. Physical activity levels of the world population: surveillance progress, gaps and prospects. Lancet. 2012;380:247-57.

7. Janssen I, LeBlanc AG. Systematic review of the health benefits of physical activity and fitness in school-aged children and youth. Int J of Behav Nutr Phys Act. 2010;7(40).

8. Ekelund U, Luan J, Sherar LB, Esliger DW, Griew P, Cooper AR. Moderate to vigorous physical activity and sedentary time and cardiometabolic risk factors in children and adolescents. JAMA. 2012;307:704-12.

9. Biddle SJH, Avare M. Physical activity and mental health in children and adolescents: a review of reviews. Br J Sports Med. 2011;45:886-95.

10. Jago R, Wedderkopp N, Kristensen PL, et al. Six-year change in youth physical activity and effect on fasting insulin and HOMA-IR. Am J Prev Med. 2008;35:554-60. 
11. Andersen LB, Harro M, Sardinha LB, et al. Physical activity and clustered cardiovascular risk in children: a cross-sectional study (The European Youth Heart Study). Lancet. 2006;368:299-304.

12. Bao WH, Srinivasan SR, Wattigney WA, Berenson GS. Persistence of multiple cardiovascular risk clustering related to syndrome $\mathrm{X}$ from childhood to young adulthood: the Bogalusa Heart Study. Arch Intern Med. 1994;154:1842-7.

13. American Public Health Association. Promoting active transportation: an opportunity for public health. Washington, DC: American Public Health Association; 2012 [cited 2013 April 24]. Available from: http://www.apha.org/NR/rdonlyres/42FBB4CA-4E2A-4C74BDD7-317E7C814F9B/0/Links Final Active Primer singles.pdf

14. British Medical Association. Healthy transport = healthy lives. London: British Medical Association; 2012 [cited 2013 April 24]. Available from: http://bma.org.uk/transport

15. Larouche R, Saunders T, Faulkner GEJ, Colley RC, Tremblay MS. Associations between active school transport and physical activity, body composition and cardiovascular fitness: a systematic review of 68 studies. J Phys Act Health. In press.

16. Lubans DR, Boreham CA, Kelly P, Foster CE. The relationship between active travel to school and health-related fitness in children and adolescents: a systematic review. Int J Behav Nutr Phys Act. 2011;8(5).

17. Shephard RJ. Is active commuting the answer to population health? Sports Med. 2008;38:751-8.

18. Andersen LB, Wedderkopp N, Kristensen P, Moller NC, Froberg K, Cooper AR. Cycling to school and cardiovascular risk factors: a longitudinal study. J Phys Act Health. 2011;8:1025-33. 
19. Chillón P, Ortega FB, Ruiz JR, et al. Bicycling to school is associated with improvements in physical fitness over a 6-year follow-up period in Swedish children. Prev Med. 2012;55:108-12.

20. Pizarro AN, Ribeiro JC, Marques EA, Mota J, Santos MP. Is walking to school associated with improved metabolic health? Int J Behav Nutr Phys Act. 2013;10(12).

21. Bryan S, St-Denis M, Wojtas D. Canadian Health Measures Survey: Clinic operations and logistics. Health Reps. 2007;18(Suppl.):53-70.

22. Day B, Langlois R, Tremblay M, Knoppers M. Canadian Health Measures Survey: Ethical, legal and social issues. Health Reps. 2007;18(Suppl.):35-52.

23. Giroux S. Canadian Health Measures Survey: sampling strategy overview. Health Reps. 2007;18(Suppl.):31-6.

24. Tremblay MS, Wolfson M, Connor Gorber S. Canadian Health Measures Survey: rationale, background and overview. Health Reps. 2007;18(Suppl.):7-20.

25. Statistics Canada. Canadian Health Measures Survey (CHMS) Data User Guide: Cycle 1. Ottawa, ON: Statistics Canada; 2011 [Cited March 26, 2013]. Available from: http://search1.odesi.ca/documentation/PHIRN/CHMS/5071 D2 T1 V1-eng.pdf

26. Evenson K, Catellier DJ, Gill K, Ondrak KS, McMurray RG. Calibration of two objective measures of physical activity for children. J Sport Sci. 2008;26:1557-65.

27. Puyau MR, Adolph AL, Vohra FA, Zakeri I, Butte NF. Prediction of activity energy expenditure using accelerometers in children. Med Sci Sports Exerc. 2004;36:1625-31. 28. Canadian Society for Exercise Physiology. The Canadian Physical Activity, Fitness \& Lifestyle Approach. $3^{\text {rd }}$ ed. Ottawa, On: Canadian Society for Exercise Physiology, 2003. 
29. Tremblay MS, Shields M, Laviolette M, Craig CL, Janssen I, Connor Gorber S. Fitness of Canadian children and youth: results from the 2007-2009 Canadian Health Measures Survey. Health Reps. 2010;21(1):7-20.

30. Bryan S, Saint-Pierre Larose M, Campbell N, Clarke J, Tremblay MS. Resting blood pressure and heart rate measurement in the Canadian Health Measures Survey, cycle 1. Health Reps. 2010;21(1):71-8.

31. Colley RC, Connor Gorber S, Tremblay MS. Quality control and data reduction procedures for accelerometry-derived measures of physical activity. Health Rep. 2010;21(1):63-9.

32. Ekelund U, Brage S, Froberg, et al. TV viewing and physical activity are independently associated with metabolic risk in children: the European Youth Heart Study. PLoS Med. $2006 ; 3: 2449-57$.

33. Rao JNK, Wu CFJ, Yue K. Some recent work on resampling methods for complex surveys. Methodology. 1992;18(2):209-17.

34. Rust KF, Rao JNK. Variance estimation for complex surveys using replication techniques. Stat Methods Med Res. 1986;5:281-310.

35. McDonald NC. Is there a gender gap in school travel? An examination of US children and adolescents. J Transp Geogr. 2012;20:80-86.

36. Pabayo R, Gauvin L, Barnett TA. Longitudinal changes in active transportation to school in Canadian children aged 6 through 16 years. Pediatrics. 2011;128(2):e404-413.

37. Panter JR, Jones AP, van Sluijs EMF. Environmental determinants of active travel in youth: a review and framework for future research. Int J Behav Nutr Phys Act. 2008;5(34). 
38. Sallis JF, Prochaska JJ, Taylor WC. A review of correlates of physical activity of children and adolescents. Med Sci Sports Exerc. 2000;32(5):963-75.

39. Holm S. A simple sequentially rejective multiple test procedure. Scand J Stat. 1979;6:6570.

40. Buliung RN, Mitra R, Faulkner G. Active school transportation in the Greater Toronto area, Canada: an exploration of trends in space and time (1986-2006). Prev Med. 2009;48:507-12.

41. Evenson KR, Huston SL, McMillen BJ, Bors P, Ward DS. Statewide prevalence and correlates of walking and bicycling to school. Arch Pediatr Adolesc Med. 2003;157:88792.

42. Faulkner GEJ, Buliung RN, Flora PK, Fusco C. Active school transport, physical activity levels and body weight of children and youth: a systematic review. Prev Med. 2009;49:38.

43. Gropp K, Pickett W, Janssen I. Multi-level examination of correlates of active transportation to school among youth living within 1 mile of their school. Int J Behav Nutr Phys Act. 2012;9(124).

44. Janssen I, Boyce WF, Simpson K, Pickett W. Influence of individual- and area-level measures of socioeconomic status on obesity, unhealthy eating, and physical inactivity in Canadian adolescents. Am J Clin Nutr. 2006;83:139-45.

45. Stamatakis E, Wardle J, Cole TJ. Childhood obesity and overweight trends in England: evidence for growing socioeconomic disparities. Int J Obes. 2010;34:41-7.

46. Pucher J, Buehler R. Making cycling irresistible: Lessons from the Netherlands, Denmark and Germany. Transp Rev. 2008;28:495-528. 
47. Cooper AR, Wedderkopp N, Jago R, et al. Longitudinal associations of cycling to school with adolescent fitness. Prev Med. 2008;47:324-8.

48. Børrestad LAB, Østergaard L, Andersen LB, Bere E. Experiences from a randomized controlled trial on cycling to school: does cycling increase cardiorespiratory fitness. Scand J Public Health. 2012;40:245-52.

49. Andersen LB, Lawlor DA, Cooper AR, Froberg K, Andersen SA. Physical fitness in relation to transport to school in adolescents: the Danish youth and sports study. Scand J Med Sci Sports. 2009;19:406-11.

50. Gordon-Larsen P, Boone-Heinonen JE, Sidney S, Sternfeld B, Jacobs DR Jr., Lewis CE. Active commuting and cardiovascular disease risk: The CARDIA study. Arch Intern Med. 2009;169:1216-23.

51. Hamer M, Chida Y. Active commuting and cardiovascular risk: a meta-analytic review. Prev Med. 2008;46:9-13.

52. Hu G, Sarti C, Jousilahti P, Silventoinen K, Barengo NC, Tuomilehto J. Leisure time, occupational, and commuting physical activity and the risk of stroke. Stroke. 2005;36:1994-9.

53. Andersen LB, Schnohr P, Schroll M, Hein HO. All-cause mortality associated with physical activity during leisure time, work, sports, and cycling to work. Arch Intern Med. 2000;160:1621-8.

54. Hu G, Eriksson J, Barengo NC, et al. Occupational, leisure time, and commuting physical activity in relation to total and cardiovascular mortality among Finnish subjects with type 2 diabetes. Circulation. 2004;110:666-73. 
55. Murtagh S, Rowe DA, Elliott MA, McMinn D, Neslon NM. Predicting active school travel: the role of planned behavior and habit strength. Int J Behav Nutr Phys Act. $2012 ; 9(65)$

56. Lemieux M, Godin G. How well do cognitive and environmental variables predict active commuting? Int J Behav Nutr Phys Act. 2009;6(12).

57. Gardner B, Abraham C. Psychological correlates of car use: a meta-analysis. Transp Res Part F. 2008;11:300-11.

58. Laurencelle L, Dupuis FA. Statistical tables explained and applied. Washington, DC, World Scientific; 2002.

59. Southward EF, Page AS, Wheeler BW, Cooper AR. Contribution of the school journey to daily physical activity in children aged 11-12 years. Am J Prev Med 2012;43:201-4.

60. Corder K, Brage S, Ekelund U. Accelerometers and pedometers: methodology and clinical application. Curr Opin Clin Nutr Metabol Care, 2007;10:597-603.

61. Børrestad LAB, Andersen LB, Bere E. Seasonal and socio-demographic determinants of school commuting. Prev Med. 2011;52:133-5.

62. Mitra R, Faulkner G. There's no such thing as bad weather, just the wrong clothing: climate, weather and active school transportation in Toronto, Canada. Can J Public Health. 2012;103(Suppl. 3):35-41.

63. Adamo K, Prince, SA, Tricco AC, Connor-Gorber S, Tremblay MS. A comparison of indirect versus direct measures for assessing physical activity in the pediatric population: a systematic review. Int J Pediatr Obes. 2009;4:2-27. 
64. Connor Gorber S, Tremblay M, Moher D, Gorber B. A comparison of direct vs. selfreport measures for assessing height, weight, and body mass index: a systematic review. Obes Rev. 2007;8:307-326.

65. Carson V, Spence JC. Seasonal variation in physical activity among children and adolescents: a review. Pediatr Exerc Sci. 2010;22:81-92. 


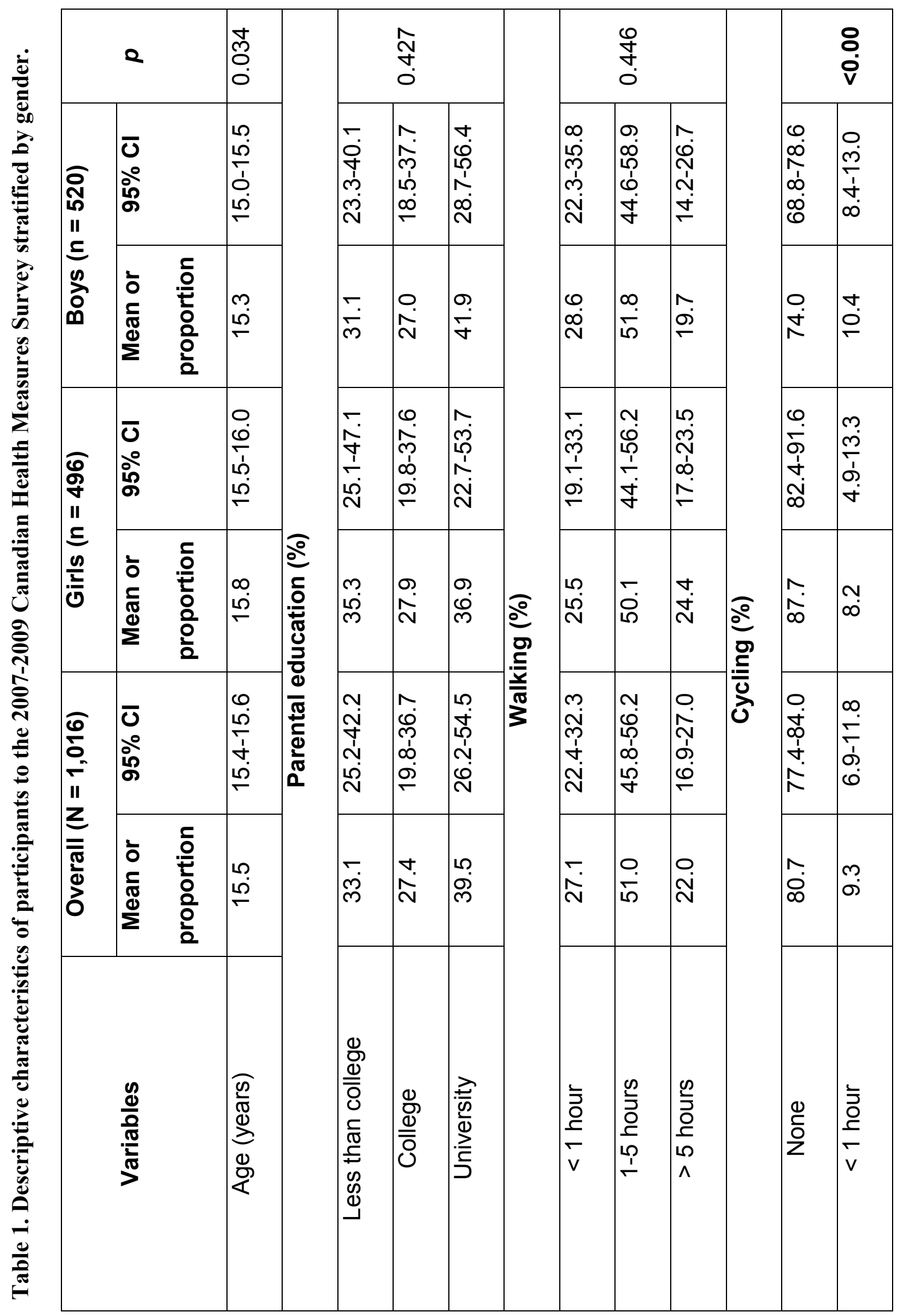




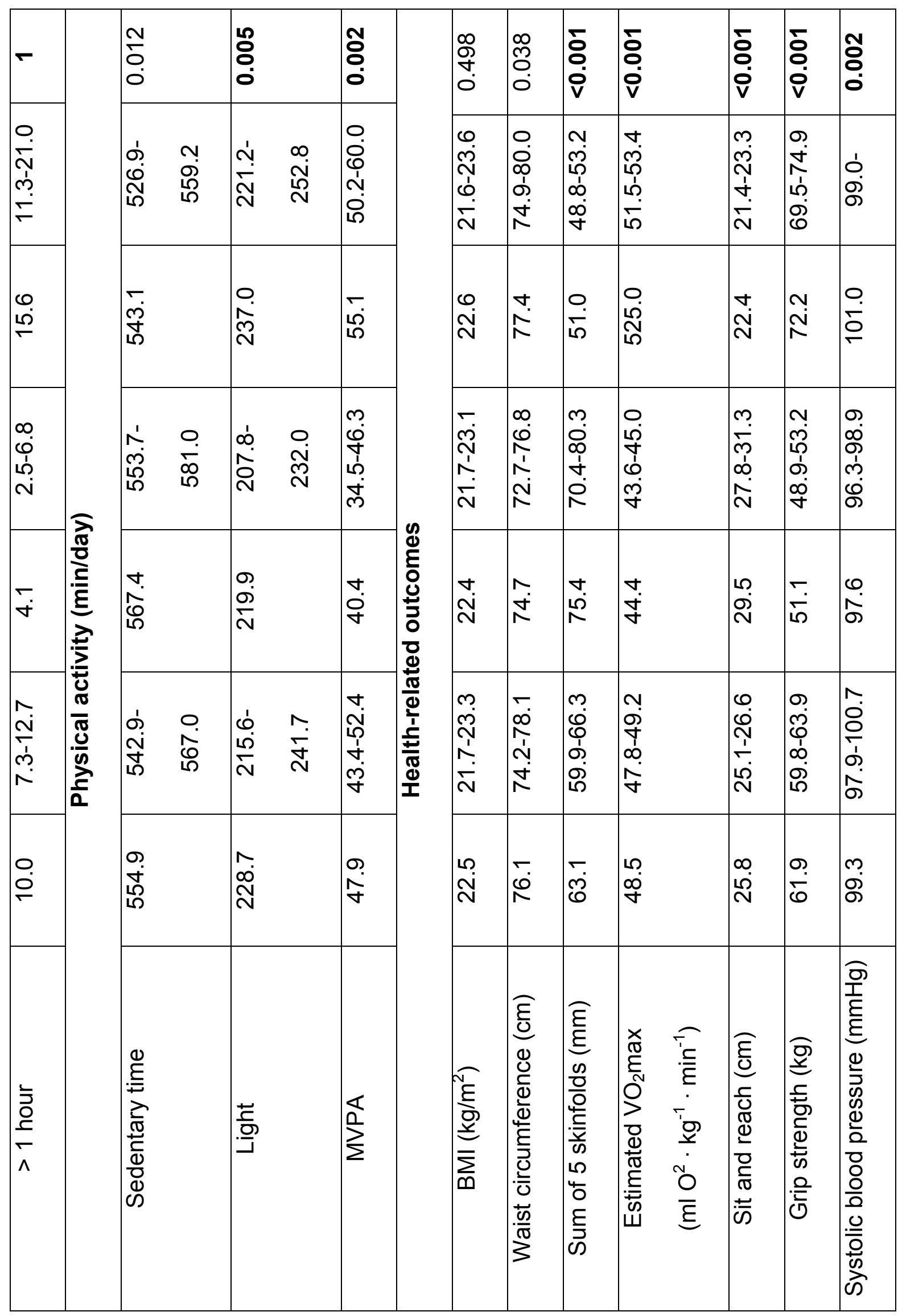




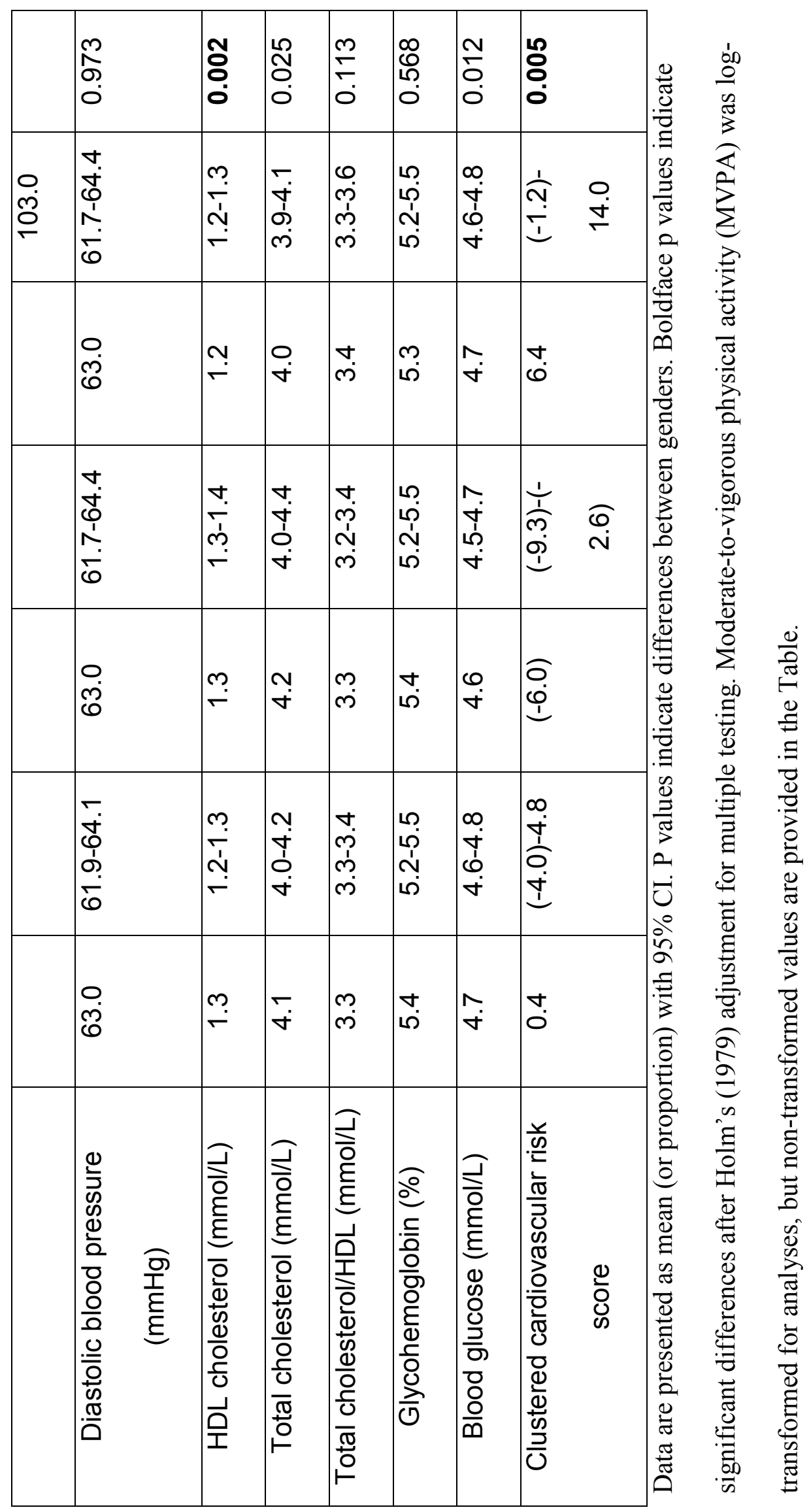


Table 2. Associations of levels of walking with gender, age group and parental education levels

\begin{tabular}{|c|c|c|c|c|c|}
\hline Variable & $<1$ hour & 1-5 hours & $>5$ hours & $\mathbf{F}$ & $p$ \\
\hline Gender & & & & 0.81 & 0.446 \\
\hline Girls & $\begin{array}{c}25.5 \\
(19.1-33.1)\end{array}$ & $\begin{array}{c}50.1 \\
(44.1-56.2)\end{array}$ & $\begin{array}{c}24.4 \\
(17.8-23.5)\end{array}$ & & \\
\hline Boys & $\begin{array}{c}28.6 \\
(22.3-35.8)\end{array}$ & $\begin{array}{c}51.8 \\
(44.6-58.9)\end{array}$ & $\begin{array}{c}19.7 \\
(14.2-26.7)\end{array}$ & & \\
\hline Age Group & & & & 9.94 & 0.001 \\
\hline $12-15$ years old & $\begin{array}{c}30.3 \\
(24.8-26.4)\end{array}$ & $\begin{array}{c}55.0 \\
(47.6-62.2)\end{array}$ & $\begin{array}{c}14.7 \\
(9.8-21.4)\end{array}$ & & \\
\hline $16-19$ years old & $\begin{array}{c}23.8 \\
(18.2-30.6)\end{array}$ & $\begin{array}{c}46.9 \\
(41.3-52.7)\end{array}$ & $\begin{array}{c}29.2 \\
(23.2-36.1)\end{array}$ & & \\
\hline Education & & & & 0.51 & 0.656 \\
\hline $\begin{array}{l}\text { Less than } \\
\text { college }\end{array}$ & $\begin{array}{c}25.0 \\
(18.9-32.2)\end{array}$ & $\begin{array}{c}54.8 \\
(44.7-64.4)\end{array}$ & $\begin{array}{c}20.2 \\
(13.5-29.2)\end{array}$ & & \\
\hline College & $\begin{array}{c}29.2 \\
(21.1-39.0)\end{array}$ & $\begin{array}{c}48.8 \\
(40.5-57.3)\end{array}$ & $\begin{array}{c}21.9 \\
(16.8-28.1)\end{array}$ & & \\
\hline University & $\begin{array}{c}29.7 \\
(20.9-40.3)\end{array}$ & $\begin{array}{c}49.3 \\
(38.4-60.2)\end{array}$ & $\begin{array}{c}21.0 \\
(15.3-28.4)\end{array}$ & & \\
\hline
\end{tabular}

Data are presented as proportions with $95 \%$ CI in parentheses. 
Table 3. Associations of levels of cycling with gender, age group and parental education levels

\begin{tabular}{|c|c|c|c|c|c|}
\hline Variable & No cycling & $<1$ hour & $>1$ hours & $\mathbf{F}$ & $p$ \\
\hline Gender & & & & 15.41 & $<0.001$ \\
\hline Girls & $\begin{array}{c}87.7 \\
(82.4-91.6)\end{array}$ & $\begin{array}{c}8.2 \\
(4.9-13.3)\end{array}$ & $\begin{array}{c}4.1 \\
(2.5-6.8)\end{array}$ & & \\
\hline Boys & $\begin{array}{c}74.0 \\
(68.8-78.6)\end{array}$ & $\begin{array}{c}10.4 \\
(8.4-13.0)\end{array}$ & $\begin{array}{c}15.6 \\
(11.3-20.9)\end{array}$ & & \\
\hline Age Group & & & & 4.24 & 0.028 \\
\hline $12-15$ years old & $\begin{array}{c}77.7 \\
(72.0-82.5)\end{array}$ & $\begin{array}{c}12.3 \\
(9.0-16.6)\end{array}$ & $\begin{array}{c}10.0 \\
(6.9-14.4)\end{array}$ & & \\
\hline $16-19$ years old & $\begin{array}{c}83.7 \\
(79.7-87.1)\end{array}$ & $\begin{array}{c}6.4 \\
(4.0-10.1)\end{array}$ & $\begin{array}{c}9.9 \\
(7.1-13.0)\end{array}$ & & \\
\hline Education & & & & 0.88 & 0.447 \\
\hline $\begin{array}{c}\text { Less than } \\
\text { college }\end{array}$ & $\begin{array}{c}78.6 \\
(73.5-83.0)\end{array}$ & $\begin{array}{c}10.8 \\
(6.8-16.7)\end{array}$ & $\begin{array}{c}10.6 \\
(7.3-15.1)\end{array}$ & & \\
\hline College & $\begin{array}{c}82.3 \\
(74.4-88.2)\end{array}$ & $\begin{array}{c}6.7 \\
(3.6-11.9)\end{array}$ & $\begin{array}{c}11.0 \\
(6.9-17.2)\end{array}$ & & \\
\hline University & $\begin{array}{c}79.7 \\
(75.3-83.5)\end{array}$ & $\begin{array}{c}10.3 \\
(7.2-14.4)\end{array}$ & $\begin{array}{c}10.0 \\
(7.1-13.9)\end{array}$ & & \\
\hline
\end{tabular}

Data are presented as proportions with $95 \%$ CI in parentheses. 


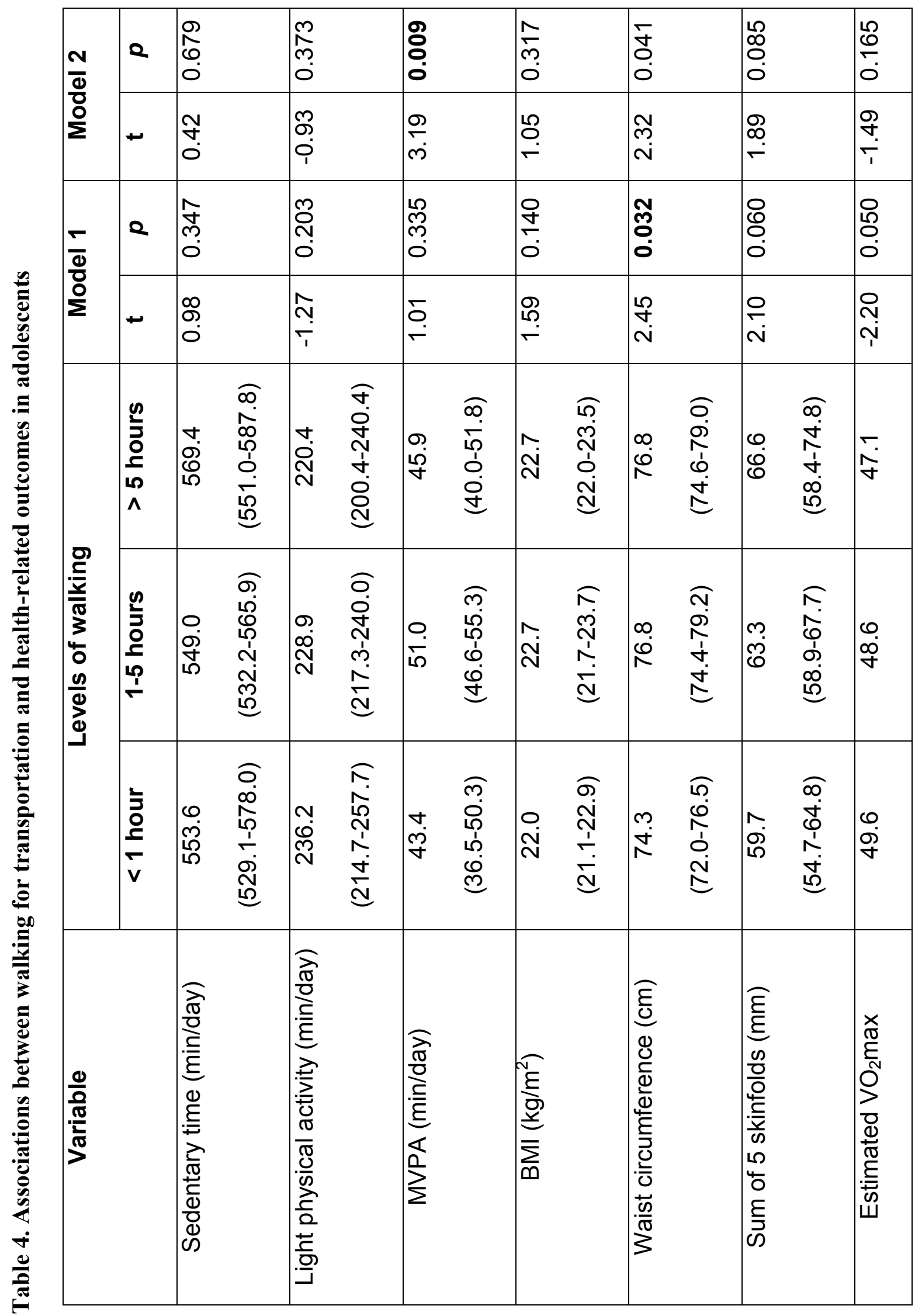




\begin{tabular}{|c|c|c|c|c|c|c|c|c|c|c|c|c|c|c|c|}
\hline & $\begin{array}{l}\hat{o} \\
0 \\
0\end{array}$ & & & & \begin{tabular}{|l}
$\bar{\delta}$ \\
$\infty$ \\
0 \\
0
\end{tabular} & & 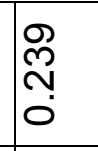 & & $\begin{array}{c}\bar{m} \\
\widetilde{m} \\
0\end{array}$ & 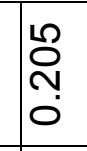 & $\begin{array}{l}\bar{\alpha} \\
0 \\
0\end{array}$ & $\begin{array}{l}\approx \\
0 \\
0 \\
0\end{array}$ & $\begin{array}{l}\mathbb{O} \\
\text { N̦ } \\
0\end{array}$ & సֶ̃ & \\
\hline & \begin{tabular}{l}
$œ$ \\
\hdashline \\
0 \\
0
\end{tabular} & & $\stackrel{\Gamma}{\Gamma}$ & & $\stackrel{\infty}{0}$ & & & & $\frac{\widetilde{\delta}}{\bar{\gamma}}$ & 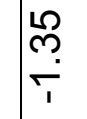 & $\frac{m}{c}$ & $\frac{\mathcal{N}}{\tau}$ & 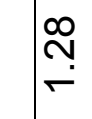 & $\stackrel{t}{r}$ & \\
\hline & $\begin{array}{l}\text { षे } \\
\stackrel{8}{0}\end{array}$ & & 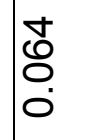 & & $\frac{d}{d}$ & & $\begin{array}{l}\mathscr{0} \\
0 \\
0 \\
0\end{array}$ & & 竜 & $\begin{array}{l}0 \\
\mathscr{D} \\
0 \\
0\end{array}$ & $\begin{array}{l}\mathscr{m} \\
\infty \\
0 \\
0\end{array}$ & $\begin{array}{l}\mathscr{O} \\
\tilde{O} \\
0\end{array}$ & $\begin{array}{l}\mathscr{0} \\
\mathscr{\delta} \\
0 \\
0\end{array}$ & $\frac{N}{\sigma}$ & \\
\hline & 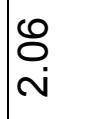 & & $\begin{array}{l}\stackrel{D}{0} \\
\text { in }\end{array}$ & & . & & 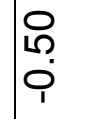 & & $\begin{array}{l}\infty \\
\infty \\
0 \\
0\end{array}$ & 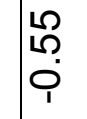 & Nָ & 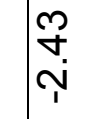 & ণ্ণ & $\stackrel{R}{r}$ & \\
\hline 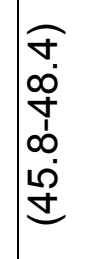 & 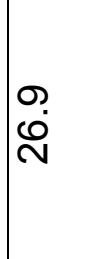 & 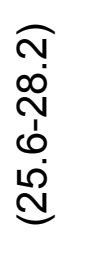 & $\begin{array}{l}\infty \\
\ddot{0} \\
\ddot{\theta}\end{array}$ & 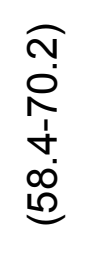 & đ̃. & $\begin{array}{l}\widehat{\infty} \\
\dot{\sigma} \\
\overline{0} \\
\dot{0} \\
\infty \\
\stackrel{\infty}{0} \\
\stackrel{0}{0}\end{array}$ & 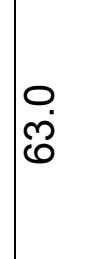 & 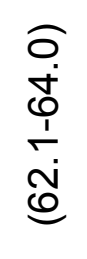 & 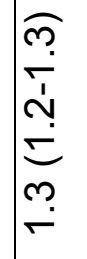 & 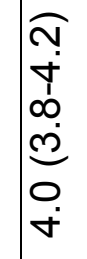 & 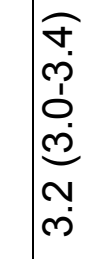 & 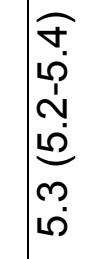 & 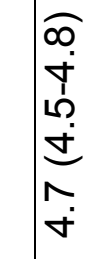 & 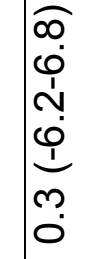 & \\
\hline 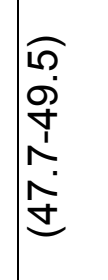 & 岕 & 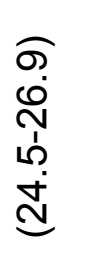 & $\begin{array}{l}0 \\
\dot{0}\end{array}$ & $\begin{array}{l}\widehat{N} \\
\dot{0} \\
0 \\
0 \\
\dot{1} \\
\hat{0}\end{array}$ & $\begin{array}{l}\text { ते } \\
\text { ه্ }\end{array}$ & 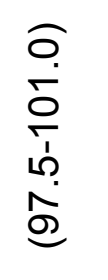 & 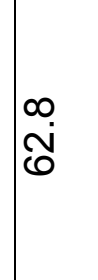 & 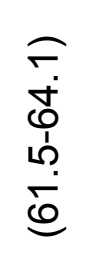 & 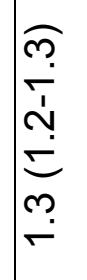 & 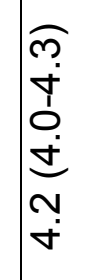 & 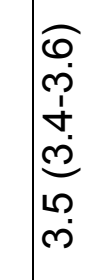 & 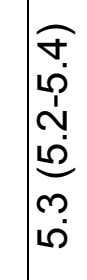 & 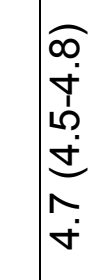 & 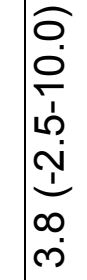 & \\
\hline 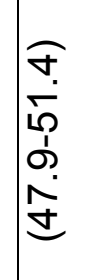 & ָึ & 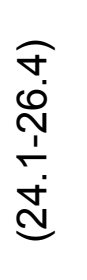 & ¿̊. & 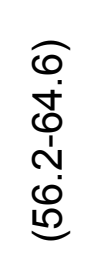 & $\begin{array}{l}\infty \\
\infty \\
\infty \\
\infty\end{array}$ & $\begin{array}{l}\widehat{0} \\
\dot{8} \\
\stackrel{0}{1} \\
\stackrel{\rho}{0} \\
\hat{0}\end{array}$ & ஜ্ঠి & $\begin{array}{l}\widehat{C} \\
\dot{0} \\
0 \\
\hat{1} \\
\dot{0}\end{array}$ & 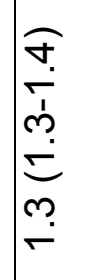 & 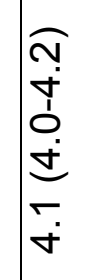 & 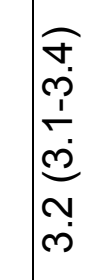 & 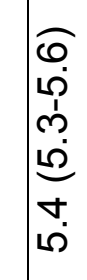 & 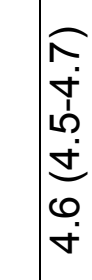 & 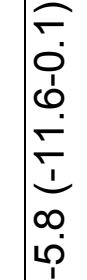 & \\
\hline 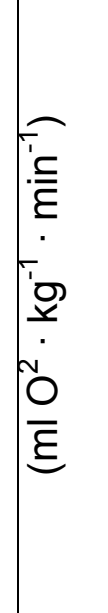 & 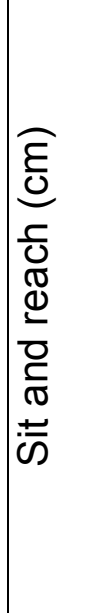 & & 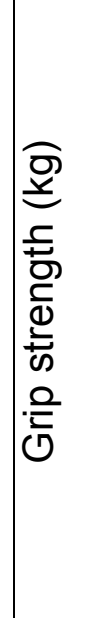 & & 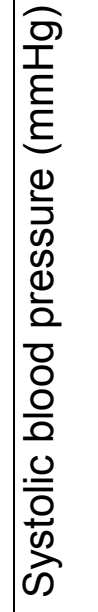 & & 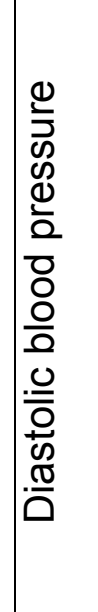 & $\begin{array}{l}\widehat{\widehat{D}} \\
\frac{1}{\xi} \\
\underline{\xi}\end{array}$ & 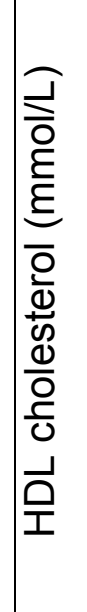 & 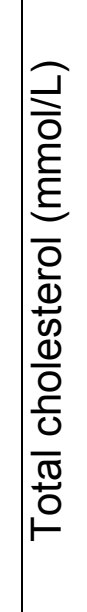 & 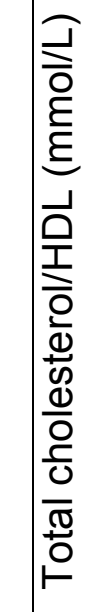 & 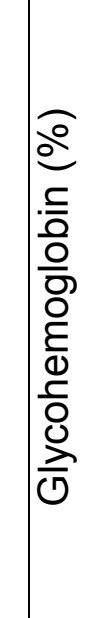 & 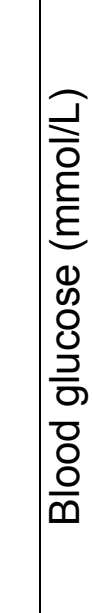 & 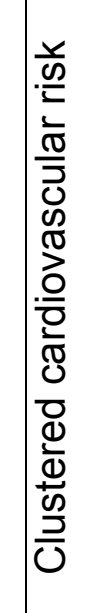 & $\begin{array}{l}0 \\
\stackrel{0}{0} \\
0\end{array}$ \\
\hline
\end{tabular}




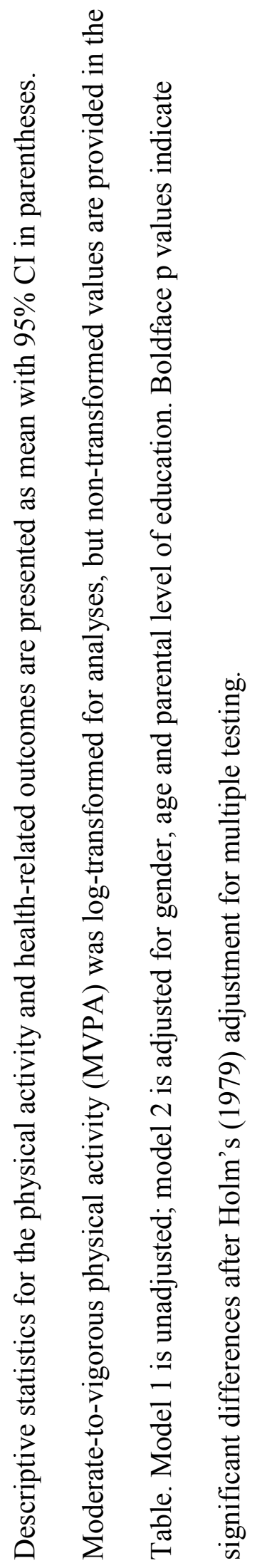

I 


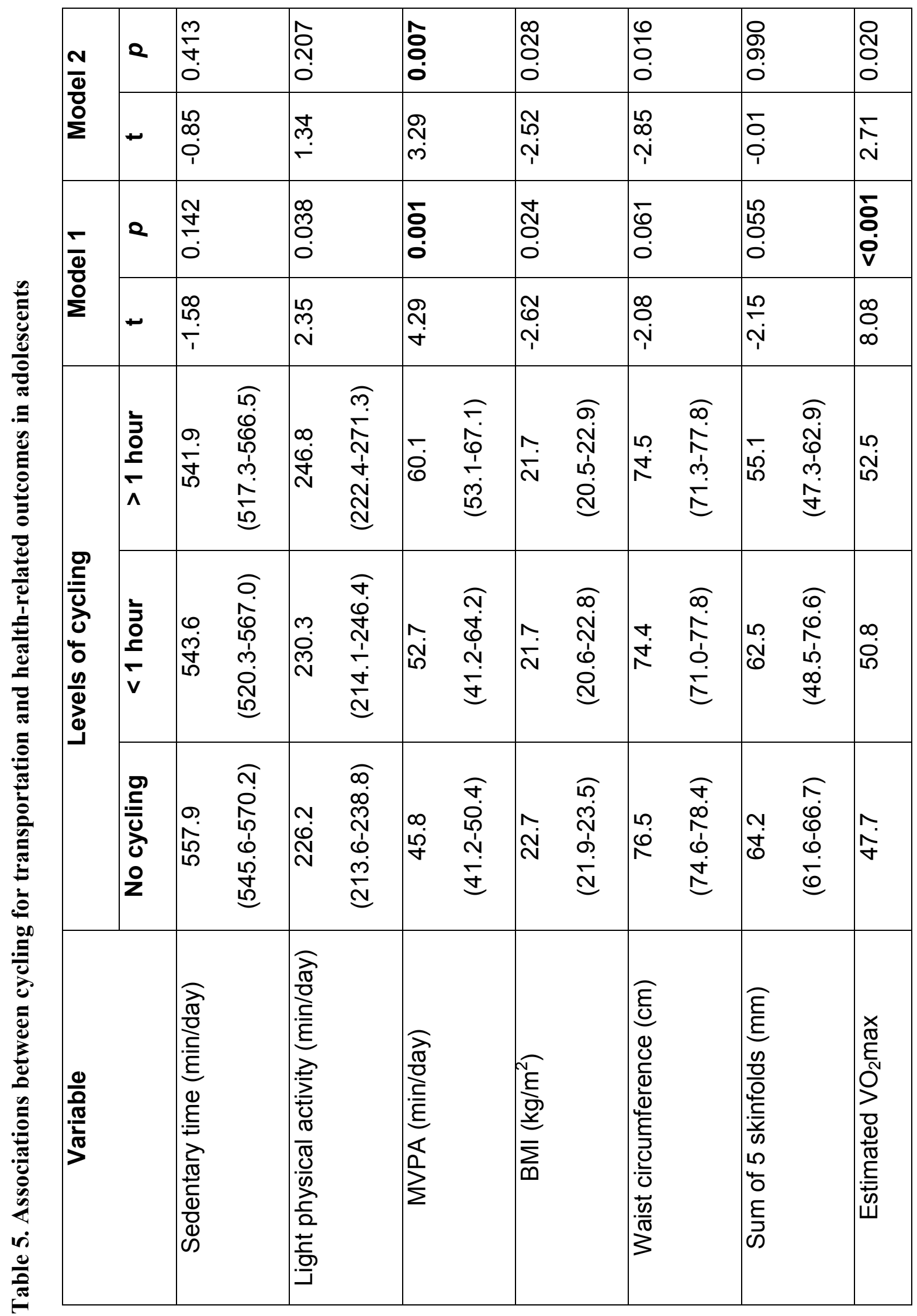




\begin{tabular}{|c|c|c|c|c|c|c|c|c|c|c|c|c|c|c|c|}
\hline & 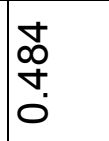 & & $\begin{array}{l}\text { D } \\
\text { O. } \\
0\end{array}$ & & $\begin{array}{l}\text { Lf } \\
\text { for } \\
0\end{array}$ & & $\begin{array}{l}0 \\
0 \\
0 \\
0\end{array}$ & & 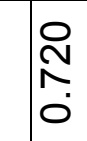 & $\begin{array}{l}0 \\
0 \\
0\end{array}$ & $\begin{array}{l}\hat{o} \\
0\end{array}$ & 总 & $\begin{array}{l}8 \\
0 \\
0 \\
0 \\
0\end{array}$ & $\begin{array}{l}\approx \\
0 \\
0 \\
0\end{array}$ & \\
\hline & רִ & & $\stackrel{\vec{S}}{\dot{r}}$ & & $\begin{array}{l}R \\
i \\
i\end{array}$ & & 吕 & & $\begin{array}{c}\hat{m} \\
0\end{array}$ & $\frac{m}{p}$ & 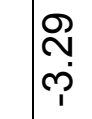 & $\underset{\mathscr{N}}{\mathscr{\sigma}}$ & ن) & 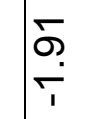 & \\
\hline & $\begin{array}{l}\frac{8}{0} \\
\vdots \\
0\end{array}$ & & $\mid \begin{array}{l}0 \\
0 \\
0 \\
0\end{array}$ & & 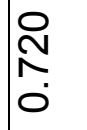 & & $\begin{array}{l}0 \\
0 \\
m \\
0 \\
0\end{array}$ & & 设 & $\begin{array}{l}\text { Oे } \\
\text { Oे }\end{array}$ & $\mid \begin{array}{l}\bar{\phi} \\
0 \\
0\end{array}$ & 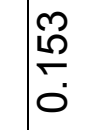 & 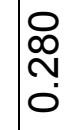 & $\begin{array}{l}\mathscr{B} \\
\stackrel{\infty}{0} \\
0\end{array}$ & \\
\hline & $\stackrel{\substack{q \\
\leftarrow}}{\leftarrow}$ & & $\begin{array}{l}\stackrel{\infty}{\infty} \\
\stackrel{\infty}{N}\end{array}$ & & $\begin{array}{l}\hat{n} \\
0 \\
0 \\
i\end{array}$ & & 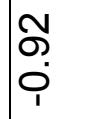 & & $\mid \begin{array}{l}\mathscr{L} \\
0 \\
0 \\
1\end{array}$ & مִ & $\frac{\mathscr{\Omega}}{\bar{\gamma}}$ & 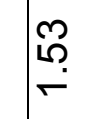 & $\stackrel{+}{\stackrel{r}{r}}$ & $\begin{array}{l}\dot{J} \\
\dot{0} \\
\dot{0}\end{array}$ & \\
\hline 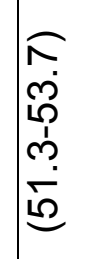 & $\begin{array}{l}\infty \\
\stackrel{N}{N}\end{array}$ & $\begin{array}{l}\widehat{T} \\
\stackrel{0}{0} \\
\infty \\
\stackrel{d}{0} \\
\end{array}$ & $\stackrel{0}{i}$ & $\begin{array}{l}\widehat{m} \\
\hat{N} \\
\infty \\
\infty \\
0 \\
0\end{array}$ & $\begin{array}{l}\circ \\
\ddot{8}\end{array}$ & $\begin{array}{l}\widehat{10} \\
\ddot{0} \\
0 \\
\dot{0} \\
\dot{0} \\
\mathscr{O}\end{array}$ & 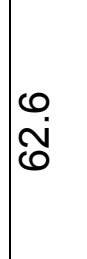 & $\begin{array}{l}\widehat{a} \\
\emptyset \\
0 \\
\vdots \\
0 \\
\dot{0}\end{array}$ & 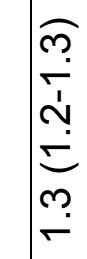 & $\begin{array}{l}\widehat{\sim} \\
\dot{\sigma} \\
\infty \\
\infty \\
\\
0 \\
0 \\
\dot{\sigma}\end{array}$ & 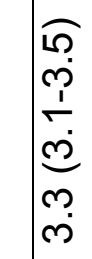 & $\begin{array}{l}10 \\
10 \\
0 \\
\sim \\
\sim \\
10 \\
0 \\
0 \\
\omega\end{array}$ & $\begin{array}{l}\widehat{\alpha} \\
\dot{j} \\
\dot{0} \\
\dot{+} \\
\hat{\sim} \\
\dot{\sigma}\end{array}$ & 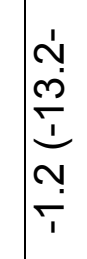 & $\begin{array}{l}\widehat{\infty} \\
\stackrel{0}{\circ}\end{array}$ \\
\hline 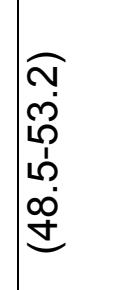 & $\stackrel{N}{N}$ & $\begin{array}{l}\text { న } \\
\infty \\
\stackrel{N}{N} \\
\stackrel{1}{\infty} \\
\stackrel{d}{d}\end{array}$ & $\frac{\dot{\nabla}}{\dot{\delta}}$ & $\begin{array}{l}\widehat{T} \\
\dot{j} \\
0 \\
0 \\
\infty \\
0 \\
0\end{array}$ & $\begin{array}{l}\infty \\
\hat{\infty}\end{array}$ & 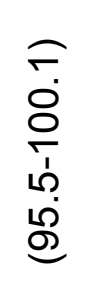 & 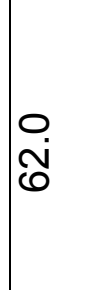 & 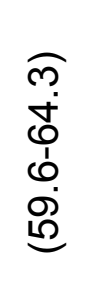 & 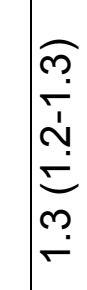 & $\begin{array}{l}\widehat{F} \\
\dot{\sigma} \\
0 \\
\dot{m} \\
0 \\
0 \\
\dot{m}\end{array}$ & 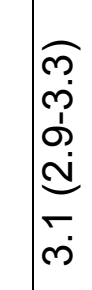 & 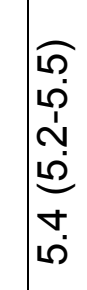 & 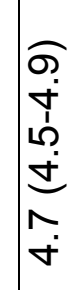 & 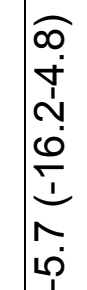 & \\
\hline 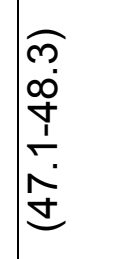 & ه্ & 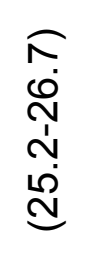 & $\begin{array}{l}8 \\
\ddot{\theta} \\
\ddot{\theta}\end{array}$ & 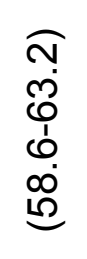 & $\begin{array}{l}10 \\
8 \\
8\end{array}$ & $\begin{array}{l}\widehat{a} \\
\dot{0} \\
\frac{0}{1} \\
\stackrel{0}{0} \\
\stackrel{0}{0}\end{array}$ & ల్n & 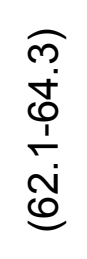 & & $\begin{array}{l}\widetilde{O} \\
\dot{J} \\
\dot{d} \\
\dot{J} \\
\check{\dot{J}}\end{array}$ & 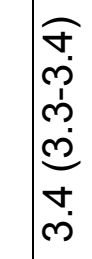 & 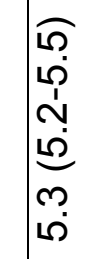 & $\begin{array}{l}\widehat{N} \\
\dot{j} \\
\dot{0} \\
\dot{f} \\
\hat{\sim} \\
\dot{\sigma}\end{array}$ & 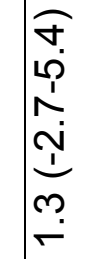 & \\
\hline 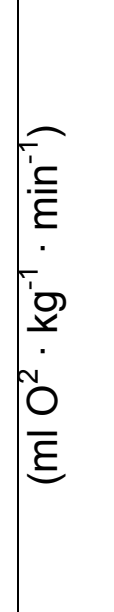 & 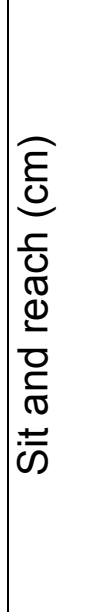 & & 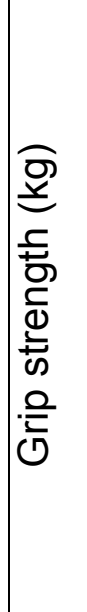 & & 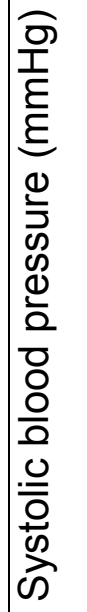 & & 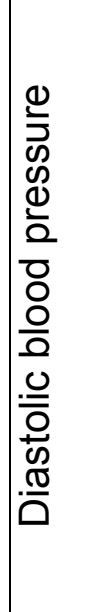 & 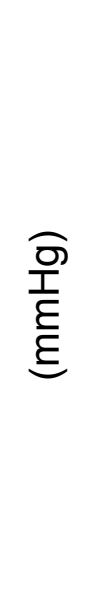 & 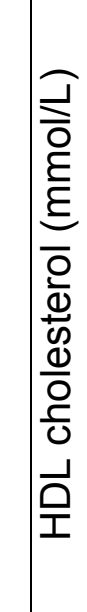 & 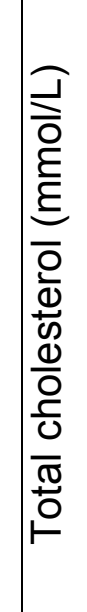 & 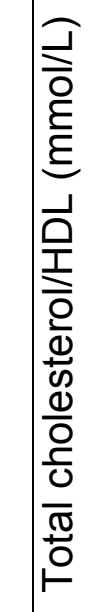 & 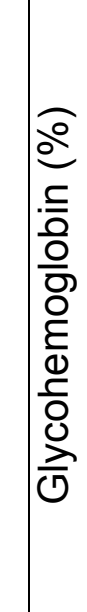 & 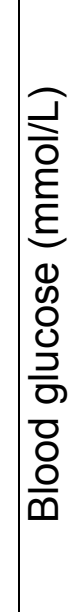 & 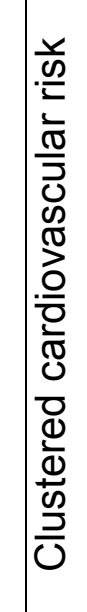 & 总 \\
\hline
\end{tabular}




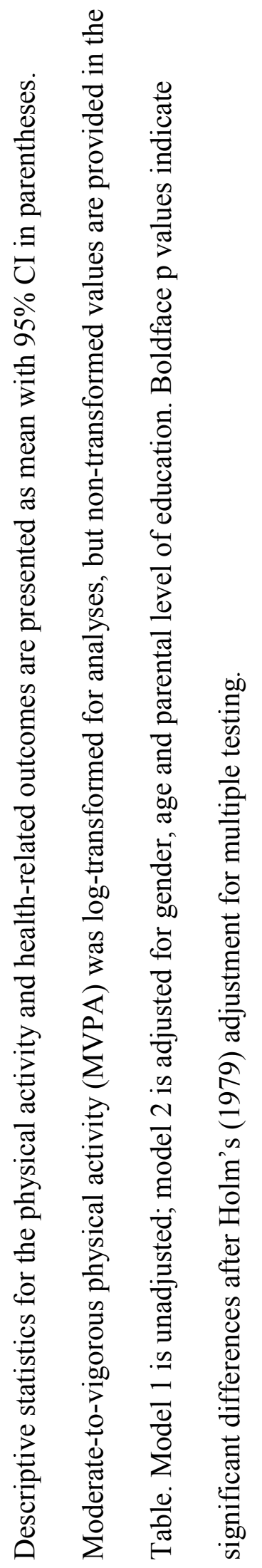


Chapter 5. Manuscript IV: "Assessing the influence of the transition from primary to secondary school on the volume of active school transport and physical activity: a prospective pilot-study"

Authors: Richard Larouche, Guy Faulkner, Mark S. Tremblay

Status: in press in Bioenergetics (2013)

Presented at: Canadian Public Health Association Conference, Ottawa, ON (June 2013). 
Title: Assessing the influence of the transition from primary to secondary school on the volume of active school transport and physical activity: a prospective pilot-study

Richard Larouche, M.Sc. ${ }^{1,2}$, Guy E. J. Faulkner, Ph.D. ${ }^{3}$, Mark S. Tremblay, Ph.D. ${ }^{1,2,4}$ 


\section{Abstract}

Objectives: Active school transport (AST, e.g. the use of non-motorized travel modes such as walking and cycling to travel to/from school) is increasingly promoted as a source of physical activity (PA) in children and youth. This prospective pilot-study assessed the: 1) test-retest reliability of a novel measure of the volume of AST; 2) changes in AST and pedometerdetermined PA across the transition from primary to secondary school; and 3) associations between AST and PA at both time points.

Methods: 55 grade 6 students were recruited from 4 primary schools in Ottawa (Canada) in May/June 2012. They were asked to complete a diary indicating their mode of transport to/from school for 1 week and wear a SC-StepMX pedometer for 8 consecutive days. 48 study packages were returned at baseline and 29 at follow-up (September/October 2012). For the test-retest assessment, a separate sample of 22 participants completed the diary during 2 consecutive weeks.

Results: The weekly volume of AST (e.g. number of active trips X distance) showed high testretest reliability $(\mathrm{ICC}=0.87)$. There were significant decreases in the proportion of children categorized as active travelers (57\% to $46 \%)$ and in step counts $(16,578 \pm 3,758$ to $14,071 \pm 3,680$ steps/day) across the school transition. However, in participants reporting at least 1 active trip at both time points $(n=11)$, the volume of AST increased with a moderate effect size $(\mathrm{d}=0.52)$, but this change was not statistically significant. While no dose-response association between the volume of AST and PA was evident (probably due to limited statistical power), a genderadjusted ANOVA indicated that active travelers accumulated an additional 2,207 steps/day at follow-up. 
Conclusion: These findings suggest that future research is needed to quantify changes in AST across the school transition and determine if AST can attenuate the commonly-observed decline in PA levels from childhood to adolescence.

Keywords: active travel, physical activity, pedometers, longitudinal study, school transition 


\section{Introduction}

In the last decade, there has been increased research on the use of modes of transport such as walking and cycling to travel to and from school (e.g. active school transport or AST) [13]. A recent systematic review reported that AST was associated with greater physical activity (PA) levels and the quality of evidence was rated as moderate [4]. This review also found that children who cycled to/from school consistently had greater cardiovascular fitness. Prospective findings from the Danish arm of the European Youth Heart Study indicated that adolescents who cycled to/from school had lower cardiovascular disease risk factors at 15 years of age [5]. Furthermore, substituting motorized trips with active travel has several environmental cobenefits including reduced greenhouse gases and particulate matter emissions and reduced traffic congestion $[6,7]$.

Despite these benefits, time trends studies have consistently shown decreases in the rates of AST in many countries including Australia [8], Canada [9], and the United States [10]. However, longitudinal studies that examined changes in AST from childhood to adolescence have reported mixed findings. Cooper and colleagues [11] observed that fewer British children walked to/from school in secondary school; however those who did so traveled greater distances. A Canadian study found that the proportion of children using AST increased with age up to 10 years of age, and then decreased afterwards [12]. In contrast, an Australian study has shown that the number of weekly trips using AST increased between the ages of 12 and 14 [13] and a Belgian study found that the proportion of participants who cycled to school was significantly lower at 10 years of age compared to $11,12,13$ and 16 years of age [14].

In parallel, prospective studies have consistently reported decreases in PA levels from childhood to adolescence $[15,16]$. However, little is known about the potential of AST to prevent 
or attenuate the decline in PA following the transition to secondary school. To our knowledge, only Cooper et al [11] have examined this question. They reported increases in moderate-tovigorous physical activity (MVPA) in British children who started to walk to school in secondary school and in those who walked at both time points. Conversely, they noted a $15.5 \%$ decrease in MVPA in children who switched from walking to car riding.

Although several studies have assessed the relationship between AST and PA levels, almost all of them have used categorical measures of AST (i.e. active vs. inactive travel) and the criteria used for categorizing individuals varied markedly across studies [4]. Another limitation of this approach is that distance traveled can moderate the relationship between AST and PA levels $[17,18]$. To our knowledge, no previous study has used a continuous measure of the volume of AST; which can be obtained by multiplying the number of active trips to/from school by the distance between individuals' home and the school that they attend.

Therefore, this pilot study aimed to address these research gaps by 1) assessing the testretest reliability of a measure of the weekly volume of AST; 2) using this instrument to assess the immediate effects of the transition from primary to secondary school (e.g. from grade 6 to grade 7) on the proportion of children engaging in AST, and the volume of AST and PA; and 3) measuring the changes in the distance between participants' home and the school that they attend, and their level of PA as measured by pedometry. Finally, the strength of the associations between measures of AST and PA was evaluated at both time points.

\section{Methods}

Participants. For the test-retest reliability component, a convenience sample of 22 primary and secondary school children (13 girls and 9 boys) aged 10 to 14 years was recruited. 
Participants were asked to complete a short diary in which they indicated their mode of transport to and from school for each day of the school week, for two consecutive weeks during the fall season.

Second, 4 K-6 primary schools in the city of Ottawa (Canada) agreed to be involved in the Active Transportation Transition Study. Only students in grade 6 were eligible to participate because the study aimed to assess the immediate influence of the school transition. Parents were asked to indicate their child's prospective secondary school for grade 7 and either their phone number or e-mail address for follow-up purposes. The flow of participants in the study is shown on Figure 1. Baseline data were collected in May/June 2012 and the follow-up was conducted in September/October 2012. Primary and secondary schools were respectively given a $\$ 100$ and $\$ 50$ honorarium for participation in the study to support physical activity-related initiatives. Ethical approval was obtained from institutional Research Ethics Boards and from the 2 participating school boards.

\section{[Insert Figure 1 here]}

Setting. It has been shown that secondary schools generally have larger catchment areas than primary schools [19]. As a result, the average distance between participant's home and the school that they attend should be greater for secondary school students, so the school transition could lead to a decrease in the mode share of AST (e.g. the proportion of children engaging in AST). According to a US study [20], average distance is generally lower in neighborhoods with greater population density; hence, population density of the primary school census tracts was obtained from the 2006 Canadian census [21]. Of the 4 schools, 2 were located in census tracts with high population density (3531-4100 inhabitants $\left./ \mathrm{km}^{2}\right)$ while the 2 others were located in lower density areas (988-2159 inhabitants $/ \mathrm{km}^{2}$ ). Participants attending primary schools located 
in higher density census tracts stayed in the same area for secondary school while those who attended primary schools located in lower density census tracts went to 7 different secondary schools, most of which were located outside of their neighborhood.

Procedures. Participants involved in the test-retest evaluation were asked to complete a short diary in which they indicated their mode of transport to and from school for each day of the school week during two consecutive weeks. Participants in the longitudinal assessment were asked to complete this diary once in the spring and once in the fall. In addition, they were instructed to wear a SC-StepMX ${ }^{\mathrm{TM}}$ pedometer (Stepscount, Deep River, ON) on the right hip for 8 consecutive days and to complete a daily log recording their daily step counts as well as the time the pedometer was worn during waking hours. This pedometer has demonstrated high validity and reliability [22]. Parents were asked to indicate their postal code and the name of their child's school to allow for estimation of the distance between home and school.

Data treatment. The volume of AST was calculated by multiplying the frequency of AST by the distance between participants' home and the school that they attend. Frequency was defined as the number of trips using active travel modes (i.e. walk, bike, skateboard, nonmotorized scooter, etc.) with values ranging from 0 to 10. Distance (in kilometers) was estimated with Google Maps, using the shortest walking route option, acknowledging that some participants may take longer routes due to safety concerns and others may take shortcuts outside of the road and sidewalks network [23]. The postal code provided by the parent was used as a proxy for residential address [24]. Because the volume of AST was skewed, it was transformed into its natural logarithm (ln) to achieve quasi-normal distribution (non-transformed descriptive data are provided in the results section for ease of interpretation). Hence, the formula was: volume $=\ln (1+$ frequency $\mathrm{X}$ distance $)$ with the constant $(1)$ added to ensure that no volume was 
below 0 . To enable comparison of the findings with other studies, a dichotomous measure of participant's primary mode of transport (e.g. active vs. inactive) was also used based on the information from the diaries. Participants were classified as active travelers if they used AST for at least $50 \%$ of school trips.

The raw pedometry data were screened based on established criteria including 1) between 1000 and 30000 steps/day [25,26]; 2) at least 10 hours of data/day [27] and 3) at least three days of valid data (e.g. meeting the daily wear threshold values) [26]. Application of these thresholds led to the exclusion of pedometer data from 2 participants at baseline and none at follow-up. The main PA measure used in the analyses was average daily steps counts, but average weekday and weekend step counts are also reported.

Statistical analyses. The test-retest reliability for the measures of primary travel mode and volume of AST were assessed with the kappa statistic and intra-class correlation coefficients (ICC) respectively. Subsequently, a series of analyses were performed. First, changes in the volume of AST, distance between home and school and average daily step counts were assessed with paired-samples t-tests, while changes in the mode share of AST were examined with a chisquare test. Second, the association of participants' primary mode of transportation with distance and PA at both time points were assessed with independent-samples t-tests and two-way (gender by travel mode) ANOVAs respectively. Third, among participants who reported at least one active trip, associations between the volumes of AST and PA were assessed with linear regression analyses adjusted for gender. Effect sizes were determined with Cohen's d for the ttests and with the partial eta-squared $\left(\eta^{2}\right)$ statistic for the ANOVAs using Cohen's [28] cutpoints. All analyses were performed with IBM SPSS 20 and $\alpha$ was set at 0.05 . 


\section{Results}

Based on the diary, the ICC for the volume of AST was 0.87 (95\% CI=0.71-0.94). The proportion of participants classified as active travelers (e.g. who did $\geq 50 \%$ of their school trips using AST) was $22.7 \%$ in week 1 and $31.8 \%$ in week 2 . The kappa value was 0.77 . Descriptive characteristics of the participants at baseline and follow-up are provided in Table 1. At baseline, 30 participants did at least one active trip accumulating an average volume of $6.9 \pm 4.5 \mathrm{~km} / \mathrm{week}$. Of the 27 participants classified as active travelers, 11 walked, 10 cycled, 2 used non-motorized scooters, 1 skateboarded and 3 used a combination of active modes. At follow-up, the average volume of AST among the 13 participants who did at least one active trip was $11.1 \pm 10.9$ km/week. Similarly, 13 participants were classified as active travelers ( 9 walked, 3 cycled and 1 used a non-motorized scooter).

\section{[Insert Table 1 here]}

The subsample of participants included in longitudinal analyses (e.g. on the basis that they provided valid data at baseline and follow-up) varied by outcome (Table 2). Active travelers tended to accumulate greater volumes of AST at follow-up, but this difference was not statistically significant $(\mathrm{t}=1.08 ; p=0.31)$ despite a moderate effect size $(\mathrm{d}=0.52)$. It is worth noting that there was large between-individual variability in the volume of AST, especially at follow-up. In contrast, the mode share of AST significantly decreased from $57 \%$ to $46 \%$ $\left(\mathrm{X}^{2}=3.88 ; p=0.05\right)$ while average distance between home and school increased from $2.6 \pm 2.7$ to $3.9 \pm 3.4 \mathrm{~km}(\mathrm{t}=-3.12 ; p=0.004)$. Average daily step counts also decreased from $16,578 \pm 3,758$ to $14,071 \pm 3,680$ steps/day $(\mathrm{t}=4.43 ; p<0.001)$. Similar declines in step counts were found for weekday and weekend days steps (data not shown).

[Insert Table 2 here] 
Two way ANOVAs examined the association between participant's primary mode of transport and step counts at both time points (Table 3). At baseline, active travelers accumulated 1,118 more steps/day than passive travelers, but this difference was not significant $(\mathrm{F}=2.17$; $p=0.15)$ and effect size was small $\left(\eta^{2}=0.05\right)$. At follow-up, the difference in step counts was 2,207 steps/day, and effect size was large $\left(\mathrm{F}=5.48 ; p=0.03 ; \eta^{2}=0.20\right)$. Although the gender by mode share interactions were not statistically significant $(p>0.22)$, the strength of the association between AST and step counts was greater in girls than in boys at baseline while the opposite trend was noted at follow-up. Independent-samples t-tests indicated that active travelers lived significantly closer to their school than inactive travelers at baseline $(\mathrm{t}=-7.04 ; p<0.001)$ and follow-up $(\mathrm{t}=-5.84 ; p<0.001)$. However, linear regression analyses demonstrated no doseresponse associations between the volume of AST and steps counts at baseline $(\mathrm{F}=0.45 ; p=0.51)$ and follow-up ( $\mathrm{F}=0.17 ; p=0.69)$.

\section{[Insert Table 3 here]}

\section{Discussion}

The present study had three main objectives: 1) to develop a measure of the volume of AST and assess its test-retest reliability; 2) to measure the changes in AST, PA and distance between home and school resulting from the transition from primary to secondary school; and 3) to assess the strength of the relationship between AST and PA at the end of primary school and the beginning of secondary school.

Test-retest reliability. High test-retest reliability coefficients were found for the participants' volume of AST and primary travel mode dichotomized as active vs. inactive. To our knowledge, no peer-reviewed study has reported the reliability of a continuous measure that 
combines both the frequency of AST trips and the distance between home and school. However, high reliability coefficients have been reported for questions on habitual travel mode and "handsup" classroom surveys [29-31].

The potential advantages of using a continuous measure may include: 1) a more precise quantification of the relationship between AST and health-related outcomes; 2) the possibility of examining dose-response relationships; and 3) using a consistent measure of AST would facilitate the inclusion of studies in meta-analyses. Indeed, meta-analyses could not be performed in earlier systematic reviews due to important discrepancies in the classification of participants as active and passive travelers $[2,4]$. Other studies also suggest that the criterion distances for walking and cycling (e.g. the distance that one would be willing to walk or cycle) vary between countries and between age groups [32-34]. While the present study was likely underpowered for the purpose of detecting a dose-response association, it is noteworthy that British researchers have reported that distance moderates the association between AST and PA $[17,18]$. In these 2 studies, distance was treated as a categorical variable.

\section{Immediate effects of the transition from primary to secondary school on AST and}

PA levels. A moderate-size increase in the volume of AST was observed among active travelers; however, this difference was not statistically significant, likely owing to the large variability in the volume of AST and the small sample size. A posteriori sample size calculations based on the observed effect size indicate that 32 participants would have been necessary for a paired-samples t-test to achieve a power of 0.8 with a two-tailed $\alpha$ of 0.05 . However, only 11 participants did at least 1 active trip at both time points. Because no earlier study has reported changes in the weekly volume of AST across the transition from primary to secondary school, there is a need for future studies with larger sample sizes. 
In contrast, an $11 \%$ decrease in the mode share of AST was observed using the dichotomous measure. The lower mode share in secondary school students is consistent with findings from the Canadian National Longitudinal Survey of Children and Youth [12], although this report did not specifically assess the effect of the school transition. In line with the present study, Cooper and colleagues [11] reported a decrease in the proportion of children walking to school (from $77.0 \%$ to $60.7 \%$ ) that was mirrored by an increase in bus riding (from 0.7 to 18.6\%). Because secondary schools generally have much larger catchment areas, the proportion of students living within a "walkable" distance is likely to be lower, thereby leading to a decrease in AST [19]. A systematic review has found that distance between home and school was the most consistent environmental correlate of AST as measured by geographic information systems [23]. In the present study, the average distance increased by almost $50 \%$ across the school transition. At both time points, active travelers lived significantly closer to their school than passive travelers. Together, these findings suggest that although fewer participants used AST at follow-up, those who did so tended to travel greater distances and to accumulate greater volumes of AST.

Levels of PA decreased by about $15 \%$ across the school transition; this finding is consistent with earlier reviews $[15,16]$. For instance, in their meta-analysis of 26 tracking studies, Dumith and colleagues [15] found a 7\% annual decline in PA during adolescence and only one study reporting an increase. In a life course perspective, studies have found that the agerelated decline in PA is not linear; it tends to be faster upon key transition periods in the lifespan (such as the school transition, entry into the labour market, etc.), and slower between these major life events [35-37]. Hence, these authors argued that such major life events are critical periods for the promotion of PA. 
Association between AST and PA levels. The third objective of the study was to examine the strength of the association between AST and PA levels at both time points. Linear regression analyses found no evidence of an association between the volume of AST and PA levels at any time points. However, as stated previously, this might be explained by the large variability of the volume of AST and the small sample size. Hence, further research is needed to determine whether there is a dose-response relationship between the volume of AST and PA level.

Two-way ANOVAs indicated that active travelers accumulated 2,217 additional steps per day on weekdays at follow-up with a large effect size. However, daily step counts did not significantly differ between active and inactive travelers at baseline. This finding could be partly explained by shorter distances between home and school and by the limited ability of pedometers to measure PA during cycling [38] given that 10 participants cycled to/from school at baseline. Furthermore, primary school children using AST may have "compensated" by being less active in the remainder of the day [39]. This potential explanation is supported by the observation that active travelers accumulated only 132 more steps/day on weekdays during primary school. It is also noteworthy that inconsistencies between genders were observed with respect to the strength of the association between AST and PA. Other studies have found similar inconsistencies [4042].

British researchers recently examined the relationship between AST and PA level in primary and secondary school students using a combination of accelerometers and global positioning systems in two separate cross-sectional studies [43-44]. In line with the present study, they observed that the relationship between AST and daily MVPA was stronger in secondary school students than in primary school students. Cooper and colleagues [11] also 
found an increase in PA across the school transition in participants who started walking. Together, these studies provide preliminary evidence suggesting that promoting AST during adolescence may be a key strategy to attenuate the age-related decline in PA levels. How best to do this remains to be seen given that the majority of school travel intervention research has been done in the elementary school context [45]. Barriers and facilitators to AST may be very different for adolescents.

Limitations and strengths. The small sample size should be considered when interpreting the findings for the following reasons. First, limited statistical power may have reduced the likelihood of observing significant associations; thus caution is needed in making a distinction between a lack of evidence of effect and evidence of no effect. Second, the sample may not be representative of the population, especially given the low participation rate. The longitudinal study design, and the school board requirement that incentives cannot be offered to participants, may have contributed to the low participation rate. Third, the volume of AST was calculated irrespective of active travel mode, although it is known that pedometers underestimate PA during cycling [38]. Because cyclists generally traveled longer distances, combining active travel modes in the analyses may have reduced the likelihood of observing significant relationships. Thus, whenever possible, future studies should examine active travel modes separately, as recommended by Shephard [3]. In addition, the observed decline in PA may be partially explained by seasonal variations [46,47]. However, the follow-up was done as early as possible in the school year in order to minimize this potential bias. Alternatively, conducting the follow-up in the following spring would have avoided seasonal bias, but at the expense of less accurately measuring the immediate effects of the school transition, which was the main study objective. Interestingly, no seasonal differences in travel modes were observed among 11-12 
years old children in Toronto [48]. Finally, the use of postal codes rather than actual street addresses limits the precision of the distance estimation, but there is no reason to believe that it has systematically biased the findings in one direction or another. A study in Calgary (Canada) found that $87.9 \%$ of postal code locations were within 200 meters of the true address location [24].

On the other hand, the prospective study design is an important strength of the study. Second, while the school transition had repeatedly been shown to be associated with a major decrease in PA [15], only one study had previously examined changes in AST upon this important transition period [11]. Third, PA was assessed with an objective measure rather than a self-report instrument prone to recall bias [49]. Furthermore, the present study confirms the testretest reliability of a new approach to quantify the volume of AST which can be included in future studies with minimal cost.

\section{Conclusion}

This pilot-study found that one week measurement of the volume of AST is very reliable, providing a new strategy for researchers interested in quantifying the relationship between AST and health-related outcomes. Consistent with previous studies, an important decline in PA was observed upon the transition from primary to secondary school. The mode share of AST also decreased significantly during this transition, but active travelers tended to accumulate greater volumes of AST in secondary school. Furthermore, the difference in step counts between active and inactive travelers was about twice as large in secondary school. Together, these findings suggest that AST may account for a greater proportion of PA among adolescents than in children. However, these findings must be interpreted with caution given the small sample size; 
thus, there is a need for the replication of this study design with larger samples. Nevertheless, the present findings provide a rationale for future studies to determine whether the promotion of AST during adolescence could attenuate the commonly observed age-related decline in PA.

Acknowledgements. We would like to acknowledge the two school boards, the school principals, teachers and the participants for their involvement in the study. Funding was provided by the Healthy Active Living and Obesity Research Group at the Children's Hospital of Eastern Ontario Research Institute.

\section{References}

1. Davison KK, Werder JL, Lawson CT (2008) Active commuting to school: current knowledge and future directions. Prev Chronic Dis 5(3). http://www.ncbi.nlm.nih.gov/pmc/articles/PMC2483568/

2. Faulkner GEJ, Buliung RN, Flora PK, Fusco C (2009) Active school transport, physical activity levels and body weight of children and youth: a systematic review. Prev Med 48(1): 3-8. http://www.ncbi.nlm.nih.gov/pubmed/19014963

3. Shephard RJ (2008) Is active commuting the answer to population health? Sports Med 38(9): 751-758. http://www.ncbi.nlm.nih.gov/pubmed/18712942

4. Larouche R, Saunders T, Faulkner GEJ, Colley RC, Tremblay MS (in press) Associations between active school transport and physical activity, body composition and cardiovascular fitness: a systematic review of 68 studies. J Phys Act Health. http://www.ncbi.nlm.nih.gov/pubmed/23250273 
5. Andersen LB, Wedderkopp N, Kristensen P, Moller NC, Froberg K, Cooper AR (2011) Cycling to school and cardiovascular risk factors: a longitudinal study. J Phys Act Health 8: 1025-1033. http://www.ncbi.nlm.nih.gov/pubmed/22039135

6. de Nazelle A, Nieuwenhuijsen MJ, Anto JM, Brauer M, Briggs D, et al. (2011) Improving health through policies that promote active travel: A review of evidence to support integrated health impact assessment. Environ Int 37(4): 766-777. http://www.ncbi.nlm.nih.gov/pubmed/21419493

7. Marshall JD, Wilson RD, Meyer KL, Rajangam SK, McDonald NC, Wilson EJ (2010) Vehicle emissions during children's school commuting: impact of education policy. Environ Sci Technol 44(5): 1537-1543. http://www.ncbi.nlm.nih.gov/pubmed/20108961

8. van der Ploeg HP, Merom D, Corpuz G, Bauman AE (2008) Trends in Australian children traveling to school 1971-2003: burning petrol or carbohydrates? Prev Med 46(1): 60-62. http://www.ncbi.nlm.nih.gov/pubmed/17628653

9. Buliung RN, Mitra R, Faulkner G (2009) Active school transportation in the Greater Toronto area, Canada: an exploration of trends in space and time (1986-2006). Prev Med 48(6): 507-512. http://www.ncbi.nlm.nih.gov/pubmed/19272403

10. McDonald NC (2007) Active commuting to school: trends among US schoolchildren 1969-2001. Am J Prev http://www.ncbi.nlm.nih.gov/pubmed/17533067

11. Cooper AR, Jago R, Southward EF, Page AS (2012) Active travel and physical activity across the school transition: the PEACH project. Med Sci Sports Exerc 44: 1890-1897. http://www.ncbi.nlm.nih.gov/pubmed/22525779 
12. Pabayo R, Gauvin L, Barnett TA (2011) Longitudinal changes in active transportation to school in Canadian children aged 6 through 16 years. Pediatrics 128(2): e404-413. http://www.ncbi.nlm.nih.gov/pubmed/21727104

13. Hume C, Timperio A, Salmon J, Carver A, Giles-Corti B, Crawford D (2009) Walking and cycling to school: predictors of increases among children and adolescents. Am J Prev Med 36(3): 195-200. http://www.ncbi.nlm.nih.gov/pubmed/19162431

14. Cardon GM, Maes LRD, Haerens LL, de Bourdeaudhuij IMM (2012) Bicycling to school during the transition from childhood to adolescence: a six-year longitudinal study. Pediatr Exerc Sci 24(3): 369-383. http://www.ncbi.nlm.nih.gov/pubmed/22971554

15. Dumith SC, Gigante DP, Domingues MR, Kohl III HW (2011) Physical activity change during adolescence: a systematic review and a pooled analysis. Int J Epidemiol 40:685698. http://www.ncbi.nlm.nih.gov/pubmed/21245072

16. Sallis JF (2000) Age-related decline in physical activity: a synthesis of human and animal $\begin{array}{lllll}\text { studies. } & \text { Med } & \text { Sci } & \text { Sports } & \text { Exerc }\end{array}$ http://www.ncbi.nlm.nih.gov/pubmed/10994911

17. Panter JR, Jones AP, van Sluijs EMF, Griffin SJ (2011) The influence of distance to school on the associations between active commuting and physical activity. Pediatr Exerc Sci 23(1): 72-86. http://www.ncbi.nlm.nih.gov/pubmed/21467592

18. van Sluijs EMF, Fearne VA, Mattocks C, Riddoch C, Griffin SJ, Ness A (2009) The contribution of active travel to children's physical activity levels: Cross-sectional results from the ALSPAC study. Prev Med 48: 519-524. http://www.ncbi.nlm.nih.gov/pubmed/19272404 
19. Environmental Protection Agency (2003) Travel and environmental implications of school siting. Environmental Protection Agency, Washington, DC. http://www.epa.gov/smartgrowth/pdf/school travel.pdf

20. McDonald NC (2008) Children's mode choice for the school trip: the role of distance and school location in walking to school. Transportation 35: 23-35. http://link.springer.com/article/10.1007\%2Fs11116-007-9135-7?.LI=true\#

21. Statistics Canada (2006) Census tract profiles, 2006 Census. Statistics Canada, Ottawa, ON. [cited 2013 January 29]. Available from: http://www12.statcan.ca/censusrecensement/2006/dp-pd/prof/92-597/index.cfm?Lang=E

22. Colley RC, Barnes JD, Leblanc AG, Borghese M, Boyer C, Tremblay MS (in press) Validity of the SC-StepMX pedometer during treadmill walking and running. Appl Phys Nutr Metabol. http://www.nrcresearchpress.com/doi/abs/10.1139/apnm-2012-0321

23. Wong BYM, Faulkner G, Buliung R (2011) GIS measured environmental correlates of active school transport: A systematic review of 14 studies. Int J Behav Nutr Phys Act 8(39). http://www.ncbi.nlm.nih.gov/pubmed/21545750

24. Bow CJD, Waters NM, Faris PD, Seidel JE, Galbraith D et al. (2004) Accuracy of city postal code coordinates as a proxy for location of residence. Int J Health Geogr 3(5). http://www.ncbi.nlm.nih.gov/pmc/articles/PMC394341/

25. Rowe D, Mahar M, Raedeke TD, Lore J (2004) Measuring physical activity in children with pedometers: reliability, reactivity, and replacement of missing data. Pediatr Exerc Sci 16(4): 343-354. http://journals.humankinetics.com/pes-backissues/pesvolume16issue4november/measuringphysicalactivityinchildrenwithpedometers $\underline{\text { reliabilityreactivityandreplacementofmissingdata }}$ 
26. Tudor-Locke C, Burkett L, Reis JP, Ainsworth BE, Macera CA, Wilson DK (2005) How many days of pedometer monitoring predict weekly physical activity in adults? Prev Med 40(3): 293-298. http://www.ncbi.nlm.nih.gov/pubmed/15533542

27. Colley RC, Connor Gorber S, Tremblay MS (2010) Quality control and data reduction procedures for accelerometry-derived measures of physical activity. Health Reps 21(1): 63-69. http://www.ncbi.nlm.nih.gov/pubmed/20426228

28. Cohen J (1988) Statistical power analysis for the behavioral science. 2nd ed. Lawrence Erlbaum, Hillsdale, NJ. http://www.amazon.com/Statistical-Power-Analysis-Behavioral$\underline{\text { Sciences } / \mathrm{dp} / 0805802835}$

29. Bere E, Bjorkelund LA (2009) Test-retest reliability of a new self reported comprehensive questionnaire measuring frequencies of different modes of adolescents commuting to school and their parents commuting to work - the ATN questionnaire. Int J Behav Nutr Phys Act 6(68). http://www.ncbi.nlm.nih.gov/pmc/articles/PMC2768672/

30. Evenson KR, Neelon B, Ball SC, Vaughn A, Ward DS (2008) Validity and reliability of a school travel survey. J Phys Act Health 5(Suppl 1): S1-S15. http://www.ncbi.nlm.nih.gov/pubmed/18364515

31. McDonald NC, Dwelley AE, Combs TS, Evenson KR, Winters RH (2011) Reliability and validity of the Safe Routes to School parent and students survey. Int J Behav Nutr Phys Act 8(56). http://www.ncbi.nlm.nih.gov/pubmed/21651794

32. D’Haese S, de Meester F, de Bourdeaudhuij I, Deforche, B Cardon G (2011) Criterion distances and environmental correlates of active commuting to school in children. Int $\mathrm{J}$ Behav Nutr Phys Act 8(88). http://www.ncbi.nlm.nih.gov/pubmed/21831276 
33. Nelson NM, Foley E, O'Gorman DJ, Moyna NM, Woods CB (2008) Active commuting to school: how far is too far? Int $\mathrm{J}$ Behav Nutr Phys Act 5(1). http://www.ncbi.nlm.nih.gov/pubmed/18182102

34. Van Dyck D, de Bourdeaudhuij I, Cardon G, Deforche B (2010) Criterion distances and correlates of active transportation to school in Belgian older adolescents. Int J Behav Nutr Phys Act 7(87). http://www.ncbi.nlm.nih.gov/pubmed/21143868

35. Allender S, Huchinson L, Foster C (2008) Life-change events and participation in physical activity: a systematic review. Health Promot Int 23(2): 160-172. http://www.ncbi.nlm.nih.gov/pubmed/18364364

36. Engberg E, Alen M, Kukkonen-Harjula K, Peltonen JE, Tikkanen HO, Pekkarinen H (2012) Life events and changes in leisure-time physical activity: a systematic review. Sports Med 42: 433-437. http://www.ncbi.nlm.nih.gov/pubmed/22512413

37. Larouche R, Laurencelle L, Shephard RJ, Trudeau F (2012) Life transitions in the waning of physical activity from childhood to adult life: the Trois-Rivières study. J Phys Act Health 9: 516-524. http://www.ncbi.nlm.nih.gov/pubmed/22592870

38. Corder K, Brage S, Ekelund U (2007) Accelerometers and pedometers: methodology and clinical applications. Curr Opin Clin Nutr Metab Care 10(5): 597-603. http://www.ncbi.nlm.nih.gov/pubmed/17693743

39. Tremblay MS, Esliger DW, Tremblay A, Colley R (2007) Incidental movement, lifestyleembedded physical activity and sleep: new frontiers in physical activity assessment. Appl $\begin{array}{llll}\text { Physiol Nutr } & \text { Metabol } & \text { 32(Suppl. } & \text { 2E): }\end{array}$ http://www.ncbi.nlm.nih.gov/pubmed/18213950 
40. Abbott RA, MacDonald D, Nambiar S, Davies PSW (2009) The association between walking to school, daily step counts and meeting step targets in 5- to 17-years-old $\begin{array}{lllll}\text { Australian } & \text { children. } & \text { Pediatr } & \text { Exerc } & \text { Sci }\end{array}$ http://www.ncbi.nlm.nih.gov/pubmed/20128369

41. Cooper AR, Page AS, Foster LJ, Qahwaji D (2003) Commuting to school: are children who walk more active. Am J Prev Med 25(4): 273-276. http://www.ncbi.nlm.nih.gov/pubmed/14580626

42. Rosenberg DE, Sallis JF, Conway TL, Cain KL, McKenzie TL (2006) Active transport to school over 2 years in relation to weight status and physical activity. Obesity 14(10): 1771-1776. http://www.ncbi.nlm.nih.gov/pubmed/17062807

43. Cooper AR, Page AS, Wheeler BW, Griew P, Davis L et al. (2010) Mapping the walk to school using accelerometry combined with a global positioning system. Am J Prev Med 38(2): 178-183. http://www.ncbi.nlm.nih.gov/pubmed/20117574

44. Southward EF, Page AS, Wheeler BW, Cooper AR (2012) Contribution of the school journey to daily physical activity in children aged 11-12 years. Am J Prev Med 43(2): 201-204. http://www.ncbi.nlm.nih.gov/pubmed/22813686

45. Chillón P, Evenson KR, Vaughn A, Ward DS (2011) A systematic review of interventions for promoting active transportation to school. Int J Behav Nutr Phys Act 8(10). http://www.ncbi.nlm.nih.gov/pubmed/21320322

46. Carson V, Spence JC (2010) Seasonal variation in physical activity among children and $\begin{array}{llllll}\text { adolescents: } & \text { a } & \text { review. Pediatr } & \text { Exerc }\end{array}$ http://www.ncbi.nlm.nih.gov/pubmed/20332542 
47. Goodman A, Paskins J, Mackett R (2012) Day length and weather effects on children's physical activity and participation in play, sports, and active travel. J Phys Act Health 9: 1105-1116. http://www.ncbi.nlm.nih.gov/pubmed/22826506

48. Mitra R, Faulkner G (2012) There's no such thing as bad weather, just the wrong clothing: climate, weather and active school transportation in Toronto, Canada. Can J $\begin{array}{llll}\text { Public Health } & \text { 103(Suppl. } & 3 \text { ): }\end{array}$ http://journal.cpha.ca/index.php/cjph/article/view/3221

49. Adamo K, Prince, SA, Tricco AC, Connor-Gorber S, Tremblay MS (2009) A comparison of indirect versus direct measures for assessing physical activity in the pediatric population: a systematic review. Int $\mathrm{J}$ Pediatr Obes 4(1): 2-27. http://www.ncbi.nlm.nih.gov/pubmed/18720173 
Table 1. Descriptive characteristics of the sample at baseline and follow-up

\begin{tabular}{|c|c|c|c|}
\hline Variable & Categories & Baseline & Follow-up \\
\hline \multirow[t]{2}{*}{ Gender } & Girls & 24 & 16 \\
\hline & Boys & 24 & 13 \\
\hline \multirow{3}{*}{ Travel mode } & Active & $27(14 \mathrm{G}, 13 \mathrm{~B})$ & $13(8 \mathrm{G}, 5 \mathrm{~B})$ \\
\hline & Inactive & $19(9 \mathrm{G}, 10 \mathrm{~B})$ & $15(7 \mathrm{G}, 8 \mathrm{~B})$ \\
\hline & Missing & $2(1 \mathrm{G}, 1 \mathrm{~B})$ & $1(1 \mathrm{G})$ \\
\hline \multirow{3}{*}{ Volume of AST $(\mathrm{km})$} & Overall & $6.9 \pm 4.5$ & $11.1 \pm 10.9$ \\
\hline & Girls & $6.4 \pm 3.2$ & $8.9 \pm 4.2$ \\
\hline & Boys & $7.4 \pm 5.4$ & $14.6 \pm 17.4$ \\
\hline \multirow{3}{*}{$\begin{array}{c}\text { Distance } \\
(\mathrm{km})\end{array}$} & Overall & $2.3 \pm 2.4$ & $3.9 \pm 3.4$ \\
\hline & Girls & $2.2 \pm 2.3$ & $3.0 \pm 2.9$ \\
\hline & Boys & $2.5 \pm 2.6$ & $4.9 \pm 2.7$ \\
\hline \multirow{3}{*}{ Average steps/day } & Overall & $16,805 \pm 3,744^{*}$ & $14,071 \pm 3,680$ \\
\hline & Girls & $15,235 \pm 2,973$ & $12,728 \pm 3,301$ \\
\hline & Boys & $18,447 \pm 3,820$ & $15,415 \pm 3,662$ \\
\hline \multirow{3}{*}{ Weekday steps/day } & Overall & $17,501 \pm 3,872 *$ & $14,743 \pm 3,701$ \\
\hline & Girls & $16,025 \pm 3,093$ & $13,368 \pm 3,455$ \\
\hline & Boys & $19,045 \pm 4,064$ & $16,117 \pm 3,537$ \\
\hline \multirow{3}{*}{ Weekend steps/day } & Overall & $15,424 \pm 6,165$ & $12,206 \pm 4,409$ \\
\hline & Girls & $14,017 \pm 6,361$ & $10,828 \pm 3,159$ \\
\hline & Boys & $16,981 \pm 5,704$ & $13,372 \pm 5,072$ \\
\hline
\end{tabular}


AST $=$ Active school transport; $\mathrm{G}=$ girls; $\mathrm{B}=$ boys. Statistics are presented as mean $\pm \mathrm{SD}$. Differences between boys and girls were assessed with independent-samples t-tests and $\mathrm{X}^{2}$ tests where appropriate. Weekly volume of AST was computed only for participants reporting at least 1 active trip. $*$ denotes statistical significance $(p<0.05)$. 
Table 2. Changes in active school transport, distance between home and school, and average daily step counts upon the transition from primary to secondary school

\begin{tabular}{|c|c|c|c|c|c|c|}
\hline Variable & n* & Primary school & Secondary & Test & $p$ & $\mathrm{~d}$ \\
& & & school & statistic & & \\
\hline AST mode share & 28 & $57.1 \%$ & $46.4 \%$ & 3.88 & 0.049 & N/A \\
\hline Volume of AST $\dagger$ & 11 & $1.8 \pm 0.7$ & $2.2 \pm 0.8$ & 1.08 & 0.306 & 0.52 \\
\hline Distance & 28 & $2.6 \pm 2.7$ & $3.9 \pm 3.4$ & -3.12 & 0.004 & 0.41 \\
\hline Average steps/day & 26 & $16,578 \pm 3,758$ & $14,071 \pm 3,680$ & 4.43 & $<0.001$ & 0.67 \\
\hline
\end{tabular}

Changes in the mode share of AST were assessed with $\mathrm{X}^{2}$ test; changes in all other variables were assessed with paired-sample t-tests. ${ }^{*}$ n refers to the sub-samples of participants who provided valid data at baseline and follow-up for each variable. AST $=$ active school transport. $\dagger$ the log-transformed volume of AST was obtained with the following formula: $\ln (1+$ frequency $\mathrm{X}$ distance). 
Table 3. Differences in average daily step counts between active and inactive travelers.

\begin{tabular}{|c|c|c|c|c|c|c|}
\hline Period & Gender (n) & AT steps \pm SD & IT steps \pm SD & F & $p$ & $\eta^{2}$ \\
\hline \multirow{4}{*}{ Baseline } & Overall (43) & $17,300 \pm 3,389$ & $16,182 \pm 4,367$ & 2.17 & 0.15 & 0.05 \\
\cline { 2 - 7 } & Girls (22) & $16,037 \pm 2,931$ & $13,545 \pm 2,555$ & 4.02 & 0.06 & 0.17 \\
\cline { 2 - 7 } & Boys (21) & $18,908 \pm 3,365$ & $18,291 \pm 4,452$ & 0.13 & 0.72 & 0.01 \\
\hline \multirow{3}{*}{ Follow-up } & Overall (26) & $15,175 \pm 3,995$ & $12,968 \pm 3,101$ & 5.48 & 0.03 & 0.20 \\
\cline { 2 - 7 } & Girls (13) & $13,250 \pm 3,246$ & $11,893 \pm 3,584$ & 0.50 & 0.50 & 0.04 \\
\cline { 2 - 7 } & Boys (13) & $18,256 \pm 3,189$ & $13,639 \pm 2,794$ & 7.57 & 0.02 & 0.41 \\
\hline
\end{tabular}

Two-way ANOVA models (e.g. travel mode by gender) were used for the "overall" models and one-way ANOVA were used for the gender-specific models. AT = active travelers; IT = inactive travelers. $\eta^{2}$ indicates the independent effect size of travel mode within the models. 
Figure legends.

Figure 1. Flow of participants in the Active Transportation Transition Study. Baseline data was collected in May/June 2012 when participants were in primary school (grade 6) and followup data was collected in September/October 2012, just after participants had started secondary school (grade 7). 
Figure 1. Flow of participants in the Active Transportation Transition Study.

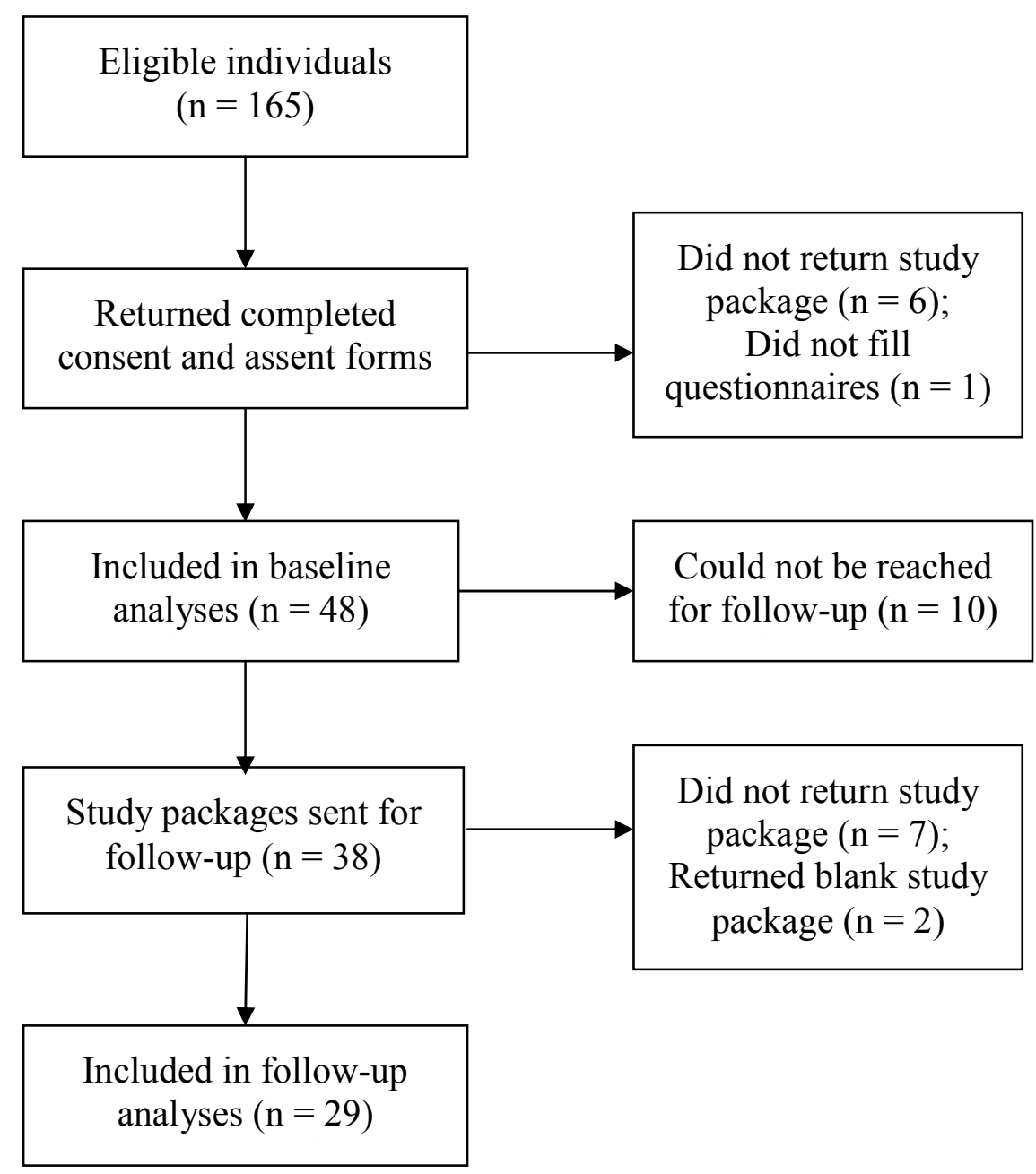


Chapter 6. Study V: "Correlates of active school travel immediately before and after the transition from primary to secondary school: a pilot-study"

Authors: Richard Larouche, Guy Faulkner, Mark S. Tremblay

Status: Accepted for publication in Journal of Applied Research on Children: Informing Policy for Children at Risk

Presented at: Canadian Public Health Association Conference, Ottawa, ON (June 2013). 


\section{INTRODUCTION}

During the last decade, active school transport (AST) has emerged as an important topic in the fields of public health, urban planning and environmental sciences. ${ }^{1-3}$ Despite the numerous benefits of AST, the proportion of children and youth using active modes of transport such as walking and cycling is generally very low and has decreased markedly in the last decades in countries such as Australia ${ }^{4}$, Canada ${ }^{5}$ and the United States ${ }^{6}$. Moreover, according to a recent systematic review, most AST promotion interventions (i.e. Safe Routes to School and walking school buses) have led to modest increases in walking and cycling to/from school and the magnitude of the observed changes has been heterogenous. ${ }^{7}$

Current theoretical models assume that parents are the key decision makers with respect to their children's travel mode ${ }^{8,9}$; hence, a large proportion of studies that examined perceived barriers to AST have focused on parents' perceptions. There is consistent evidence showing that the distance between a child's residence and the school that he/she attends (both perceived and objectively measured) is a strong determinant of travel mode choices. ${ }^{10-12}$ However, travel decisions can also be influenced by a wide array of factors pertaining to different levels of influence as postulated by social-ecological theory. 9,13 These may include personal characteristics (e.g., attitudes toward walking and cycling), the social environment (e.g., social support from family and friends), public policies (e.g., school policies, municipal bylaws), the built environment (e.g., availability of sidewalks and bicycle paths), and the physical environment (e.g. topography). These two models also posit that the relationship between the correlates of AST and travel patterns is moderated by the age of the child, but longitudinal studies on this topic are scarce. 9,13

Although children may have some input into travel mode decisions, few studies have simultaneously assessed child- and parent-perceived barriers to AST. ${ }^{9}$ Timperio and colleagues ${ }^{14,15}$ examined perceived barriers among children of different age groups and their parents, but they did not report the concordance between these perceptions. More recently, Napier and colleagues ${ }^{16}$ reported that children's and parents' perception of 5 different barriers to AST was similar, except in areas defined as "less walkable" where parents expressed greater concerns. Further research is warranted to determine the extent to which children's and parents' perceptions are concordant across a larger number of barriers pertaining to multiple levels of influence.

Another area that has been understudied is the potential role of parental neighborhood selection in influencing children's travel mode. It has been shown that adults who prefer walking and cycling are more likely to choose to live in "walkable areas". 17, 18 For example, Frank and colleagues ${ }^{17}$ assessed the relative influence of neighborhood walkability and self-selection, and they found that both aspects were independently associated with adults' travel modes. To our knowledge, only one study has examined the influence of parental neighborhood selection factors on children's travel patterns, reporting that children were more likely to engage in AST if their parents had chosen to live in their neighborhood so that their children could walk to school. ${ }^{19}$ Hence, further research is needed to confirm this association and examine the influence of other parental neighborhood selection factors; for instance parents could choose their neighborhood for its proximity to their workplace or because of monetary constraints. 
In addition, there is a growing body of evidence showing that socio-demographic characteristics can influence children's travel mode. Many North American studies have shown that girls ${ }^{20-22}$ and children living in high socioeconomic status (SES) families ${ }^{21,23 \text {, }}$ ${ }^{24}$ are less likely to walk or cycle to/from school. Other researchers have reported that car ownership was inversely related to AST ${ }^{25}$ This evidence underscores the need for studies of correlates of AST to consider socio-demographic factors.

Therefore, the present pilot-study had 4 key objectives: 1) to assess the concordance between child- and parent-perceived barriers to AST; 2) to examine the association between child- and parent-perceived barriers to AST and children's primary mode of transport; 3) to explore the influence of different parental neighbourhood selection factors on children's travel mode; and 4) to investigate the association between a series of socio-demographic characteristics of the household and AST. These relationships were assessed at the end of primary school (grade 6) and the beginning of secondary school (grade 7) among the same participants. This important transition period is associated with a large decrease in physical activity levels. ${ }^{26}$

\section{METHODS}

Participants. The principals from four K-6 primary schools agreed to be involved in the Active Transportation Transition Study. Only students in grade 6 were eligible to participate because the study aimed to assess the influence of the school transition. Consent and assent forms were obtained from 55 grade 6 students $(33.3 \%$ response rate) of which 49 returned their study package. Parents were asked to indicate their child's prospective school for grade 7 and either their phone number or e-mail address for follow-up purposes. Eleven parents could not be reached for follow-up, either because they did not complete the parent questionnaire at baseline or because they did not respond to our e-mail reminders. Of the 38 remaining participants, 31 returned their study packages, but 2 of these contained blank questionnaires; thus data from 29 participants was included in the follow-up analyses. Baseline data were collected in May/June 2012 and the follow-up data in September/October 2012. Primary and secondary schools were respectively given a $\$ 100$ and $\$ 50$ honorarium for participation in the study to support physical activity-related initiatives. Ethical approval was obtained from institutional Research Ethics Boards and from the 2 participating school boards.

Setting. It has been shown that secondary schools generally have larger catchment areas than primary schools. ${ }^{27}$ As a result, the average distance between participant's home and the school that they attend should be greater for secondary school students; thereby, the school transition could lead to a decrease in the mode share of AST (e.g. the proportion of children engaging in AST). In the subsample of participants $(n=28)$ who provided baseline and follow-up data, an increase in average distance (from $2.61 \mathrm{~km}$ to $3.86 \mathrm{~km}$ ) and a decrease in the mode share of AST (from $57.1 \%$ to $46.4 \%$ ) were observed ( $R$. Larouche, University of Ottawa, unpublished manuscript). According to a US study ${ }^{10}$, average distance is generally lower in neighborhoods with greater population density; hence, population density of the primary school census tracts was obtained from the 2006 Canadian census ${ }^{28}$. Participants attending the 2 primary schools located in higher density census tracts (3531-4100 inhabitants $/ \mathrm{km}^{2}$ ) stayed in the same area for secondary school. In contrast, those who attended the 2 primary schools located in lower density census tracts (988-2159 
inhabitants $/ \mathrm{km}^{2}$ ) went to 7 different secondary schools, most of which were located outside of their neighborhood. Assuming that distance is a key determinant of AST, one would assume that the latter participants would be less likely to engage in AST, especially in secondary school. Presumably, the relative influence of barriers to AST will be modified given the change in school location. For example, the distance to school may increase, a different set of safety concerns may present themselves, and travel modes may become more or less convenient or available (e.g., school bussing eligibility).

Measures. All assessments described below were done at baseline and followup. First, participants were asked to complete a short diary in which they indicated their mode of transport to and from school for each day during one school week. Second, participants and their parents were asked to complete a previously validated 17 -item questionnaire on perceived barriers to AST. ${ }^{29}$ In this questionnaire, individuals are asked to indicate their level of agreement with simple statements on a 4-point scale ranging from 1 (strongly disagree) to 4 (strongly agree). While the Forman et al. ${ }^{29}$ scale has been validated for assessing perceived barriers to active transport for different trip purposes, only the barriers pertaining to the school trip were examined given that the present study focused on AST. Because road safety is an important correlate of AST $^{9}$ that is specifically targeted by interventions promoting AST (i.e. Safe Routes to School), two items were added to the scale targeting traffic around participants' home and around the school that they attend. These items were added to get additional precision regarding the location of road safety concerns. Third, parental neighbourhood selection factors were assessed with a 7-item scale adapted from the Neighborhood Selection Questionnaire developed by Frank et al. ${ }^{17}$ Parents indicated their level of agreement with these statements on a 5 point-scale ranging from 1 (strongly agree) to 5 (strongly disagree). Fourth, parents were asked several socio-demographic questions including the number of children and cars in the household, whether their child had access to a bicycle, the type of family (e.g. 2 parents, 1 parent or other), and the education level and employment status of the mother and father.

Data treatment. Participants were classified as active travelers if they reported using active modes of transport for at least $50 \%$ of school trips. Pilot data using the same classification method has shown substantial test-retest reliability over two consecutive weeks of measurements (R. Larouche, University of Ottawa, unpublished manuscript). Given the small sample size, response options for the barrrier statements were dichotomized as "disagree" (strongly disagree or somewhat disagree) and "agree" (strongly agree or somewhat agree). Similarly, response options for the neighborhood selection factors were dichotomized as "disagree" (strongly disagree, somewhat disagree or neither agree nor disagree) and "agree" (strongly agree or somewhat agree). Participant's primary school neighborhood was coded as "higher" vs. "lower" density.

Statistical analyses. First, the concordance between children- and parentperceived barriers to AST was assessed using Spearman rank-order correlations. Second, Fisher's ${ }^{30}$ exact test was used to examine how children- and parent-perceived barriers, parental neighbourhood selection factors and socio-demographic factors relate to children's primary travel mode. This test is suitable for contingency tables when the 
chi-square test assumptions are not satisfied. All analyses were performed at baseline and follow-up with IBM SPSS 20 and a was set at .05.

\section{RESULTS}

Descriptive characteristics of the sample are shown in Table 1. Almost all children had access to a bicycle and almost all parents had at least 1 car. The majority of children lived in 2 parent families with highly educated parents and almost all fathers were full-time employed, suggesting that the household SES status may be higher than the average Canadian population. ${ }^{31}$ No significant differences in socio-demographic characteristics were found between individuals who participated in the follow-up and those who did not (all $p>0.232$ ).

\section{Table 1. Descriptive characteristics of the sample}

\begin{tabular}{|c|c|c|c|}
\hline Characteristic & Categories & Baseline & Follow-up \\
\hline \multirow[b]{2}{*}{ Child gender } & Girls & $25(51.0 \%)$ & $16(55.2 \%)$ \\
\hline & Boys & $24(49.0 \%)$ & $13(44.8 \%)$ \\
\hline \multirow{2}{*}{ School neighborhood } & Higher density & $23(46.9 \%)$ & $12(41.4 \%)$ \\
\hline & Lower density & $26(53.1 \%)$ & $17(58.6 \%)$ \\
\hline \multirow[b]{2}{*}{ Travel mode } & Active & $26(57.1 \%)$ & $13(46.4 \%)$ \\
\hline & Inactive & $16(42.9 \%)$ & $15(53.6 \%)$ \\
\hline \multirow{3}{*}{$\begin{array}{c}\text { Number of cars in the } \\
\text { household }\end{array}$} & 0 & $2(4.7 \%)$ & $1(3.6 \%)$ \\
\hline & 1 & $18(41.9 \%)$ & $11(39.3 \%)$ \\
\hline & 2 & $23(53.5 \%)$ & $16(57.1 \%)$ \\
\hline \multirow{2}{*}{$\begin{array}{c}\text { Children has access to a } \\
\text { bicycle }\end{array}$} & Yes & $41(95.3 \%)$ & $29(100 \%)$ \\
\hline & No & $2(4.7 \%)$ & 0 \\
\hline \multirow[b]{2}{*}{ Family type } & Two parents & $37(86.0 \%)$ & $26(89.7 \%)$ \\
\hline & Single parent & $6(14.0 \%)$ & $3(10.3 \%)$ \\
\hline \multirow{3}{*}{ Family size } & 1 children & $6(14.0 \%)$ & $4(13.8 \%)$ \\
\hline & 2 children & $25(58.1 \%)$ & $16(55.2 \%)$ \\
\hline & 3 or more children & $12(27.9 \%)$ & $9(31.0 \%)$ \\
\hline \multirow{2}{*}{ Father's education } & $\begin{array}{l}\text { Less than } \\
\text { university }\end{array}$ & $15(34.9 \%)$ & $7(25.0 \%)$ \\
\hline & University & $28(65.1 \%)$ & $21(75.0 \%)$ \\
\hline \multirow{2}{*}{ Mother's education } & $\begin{array}{l}\text { Less than } \\
\text { university }\end{array}$ & $10(23.8 \%)$ & $2(7.1 \%)$ \\
\hline & University & $32(76.2 \%)$ & $26(92.9 \%)$ \\
\hline \multirow[b]{2}{*}{ Father's employment } & Full time & $38(92.7 \%)$ & $28(96.6 \%)$ \\
\hline & Other & $3(7.3 \%)$ & $1(3.4 \%)$ \\
\hline \multirow[b]{2}{*}{ Mother's employment } & Full time & $26(65.0 \%)$ & $18(66.7 \%)$ \\
\hline & Other & $14(35.0 \%)$ & $9(33.3 \%)$ \\
\hline
\end{tabular}

Data are indicated as the number of participants with the proportion in parentheses. ${ }^{*} S c h o o l$ neighborhood refers to the school that participants attended at baseline.

Spearman correlations between child- and parent-perceived barriers to AST at baseline and follow-up are reported in Table 2. At baseline, significant correlations (rho 
0.32-0.72) were found for 14 out of 19 questionnaire items. Of particular note, correlations were generally higher for the road safety (e.g. traffic, lighting and crossings) and distance components (rho $\geq 0.50$ ). At follow-up, significant correlations between child- and parent-perceived barriers to AST (rho 0.39-0.63) were found for only 4 items: traffic along the route and around the home, crime-related concerns and distance between home and school.

Table 2. Spearman correlations between child- and parent-perceived barriers to active transportation at baseline and follow-up

\begin{tabular}{|c|c|c|c|c|}
\hline \multirow[b]{2}{*}{ Barriers } & \multicolumn{2}{|c|}{ Baseline } & \multicolumn{2}{|c|}{ Follow-up } \\
\hline & rho & $p$ & rho & $p$ \\
\hline Too many hills along the way & $.509^{*}$ & .001 & .326 & .090 \\
\hline No sidewalks or bike lanes & .155 & .341 & .247 & .206 \\
\hline Route is boring & .064 & .698 & .018 & .927 \\
\hline $\begin{array}{l}\text { Route does not have good } \\
\text { lighting }\end{array}$ & $.525^{*}$ & .001 & .380 & .050 \\
\hline Too much traffic along the route & $.721^{*}$ & $<.001$ & $.391^{*}$ & .040 \\
\hline Too much traffic around home & $.670^{*}$ & $<.001$ & $.520^{*}$ & .005 \\
\hline $\begin{array}{l}\text { Too much traffic around the } \\
\text { school }\end{array}$ & $.495^{*}$ & .001 & .247 & .214 \\
\hline $\begin{array}{c}\text { One or more dangerous } \\
\text { crossings }\end{array}$ & $.575^{\star}$ & $<.001$ & .350 & .068 \\
\hline Get too hot and sweaty & .152 & .350 & .079 & .690 \\
\hline $\begin{array}{l}\text { No other children walk or bike to } \\
\text { school }\end{array}$ & .078 & .638 & -.017 & .934 \\
\hline $\begin{array}{l}\text { Not considered cool to walk or } \\
\text { bike }\end{array}$ & $.453^{*}$ & .003 & -.067 & .736 \\
\hline Too much stuff to carry & $.684^{*}$ & $<.001$ & .182 & .353 \\
\hline $\begin{array}{l}\text { Easier for parents to drive on the } \\
\text { way to something else }\end{array}$ & $.332^{*}$ & .037 & .178 & .364 \\
\hline $\begin{array}{l}\text { Involves too much planning } \\
\text { ahead }\end{array}$ & $.320^{*}$ & .047 & .133 & .500 \\
\hline $\begin{array}{l}\text { Unsafe because of crime } \\
\text { (strangers, gangs, drugs) }\end{array}$ & $.454^{*}$ & .003 & $.425^{*}$ & .024 \\
\hline Get bullied, teased, harassed & $.546^{*}$ & $<.001$ & .104 & .598 \\
\hline Nowhere to leave a bike safely & .170 & .301 & -.026 & .899 \\
\hline There are stray dogs & $.646^{*}$ & $<.001$ & -.161 & .414 \\
\hline It's too far & $.714^{\star}$ & $<.001$ & $.629^{*}$ & $<.001$ \\
\hline
\end{tabular}

* Indicates significant correlations $(p<.05)$. The 2 items that were added to Forman and colleagues' (2008) scale are in italics.

Associations of children's primary travel mode with child- and parent-perceived barriers to AST at baseline and follow-up are reported in Table 3. At baseline, 26 participants were classified as active travelers, 19 as inactive travelers and 4 did not complete the log sheet or provided insufficient information for the determination of their 
primary travel mode. Children who perceived that the school is too far $(p<.001)$, that there is too much traffic around the school $(p=.014)$, that the route does not have good lighting $(p=.019)$ and that they have too much stuff to carry $(p=.038)$ were less likely to engage in AST. Similarly, children were less likely to engage in AST if their parents perceived that the school is too far $(p<.001)$, that their child has too much stuff to carry $(p=.005)$ or gets too hot and sweaty $(p=.015)$, that it is unsafe because of crime $(p=$ $.015)$, that there is too much traffic along the route $(p=.018)$ or the home $(p=.035)$, that the route does not have good lighting $(p=.046)$ and that no other children walk or bike to/from school $(p=.046)$. Participants who somewhat or strongly agreed that the school is too far were all inactive travelers.

At follow-up, 13 participants were classified as active travelers, 15 as inactive travelers and 1 provided insufficient information to allow for the determination of travel mode. Children were less likely to engage in AST if they perceived that the school is too far $(p=.002)$, that there are dangerous crossings along the route $(p=.006)$, that they have too much stuff to carry $(p=.018)$, that it is easier for their parents to drive them ( $p$ $=.038$ ) and that there is too much traffic around the school $(p=.044)$. Children were also less likely to engage in AST if their parent perceived that the school is too far $(p=$ $.003)$ and that there are no sidewalks or bike lanes along the route $(p=.008)$. Only one child who perceived that the school is too far engaged in AST.

Table 4 shows the association between parental neighborhood selection factors and children's travel mode at baseline and follow-up. At both time points, children were more likely to engage in AST if their parents indicated that they have chosen to live in their neighborhood because "it is easy for our children to walk or cycle to school" ( $p=$ .001 and .047 respectively). At follow-up, children were also more likely to engage in AST if their parents indicated that they have chosen to live in their neighborhood because "there are shops and restaurants you can walk or cycle to" $(p=.036)$.

The association between socio-demographic charecteristics of the household and children's travel mode at baseline and follow-up is shown in Table 5. At baseline and follow-up, children attending school in denser areas were significantly more likely to engage in AST ( $p=.003$ and $p<.001$ respectively). At follow-up only, children whose parents owned at least 2 cars were less likely to engage in AST $(p=.006)$. Other sociodemographic characteristics were not associated with children's travel mode at any time point. Because more than $95 \%$ of participants had access to a bicycle, this variable was omitted from the analyses. 
Table 3. Associations between child-perceived barriers and active school transport at baseline and follow-up

\begin{tabular}{|c|c|c|c|c|c|}
\hline Barriers & $\begin{array}{l}\text { Study } \\
\text { period }\end{array}$ & Respondent & AT $(\%)$ & IT (\%) & $p$ \\
\hline \multirow{4}{*}{$\begin{array}{l}\text { Too many hills } \\
\text { along the way }\end{array}$} & Baseline & Child & 13.0 & 17.6 & .666 \\
\hline & Baseline & Parent & 4.0 & 7.1 & 1.000 \\
\hline & Follow-up & Child & 7.7 & 33.3 & .173 \\
\hline & Follow-up & Parent & 0.0 & 26.7 & .106 \\
\hline \multirow{4}{*}{$\begin{array}{l}\text { No sidewalks or } \\
\text { bike lanes }\end{array}$} & Baseline & Child & 19.2 & 41.2 & .168 \\
\hline & Baseline & Parent & 16.0 & 40.0 & .135 \\
\hline & Follow-up & Child & 7.7 & 40.0 & .084 \\
\hline & Follow-up & Parent & 0.0 & 46.7 & $.008^{*}$ \\
\hline \multirow{4}{*}{ Route is boring } & Baseline & Child & 7.7 & 17.6 & .369 \\
\hline & Baseline & Parent & 0.0 & 7.1 & .359 \\
\hline & Follow-up & Child & 0.0 & 13.3 & .484 \\
\hline & Follow-up & Parent & 0.0 & 0.0 & N/A \\
\hline \multirow{4}{*}{$\begin{array}{l}\text { Route doesn't } \\
\text { have good } \\
\text { lighting }\end{array}$} & Baseline & Child & 0.0 & 23.5 & $.019^{*}$ \\
\hline & Baseline & Parent & 0.0 & 20.0 & $.046^{*}$ \\
\hline & Follow-up & Child & 7.7 & 14.3 & 1.000 \\
\hline & Follow-up & Parent & 8.3 & 20.0 & .605 \\
\hline \multirow{4}{*}{$\begin{array}{l}\text { Too much traffic } \\
\text { along the route }\end{array}$} & Baseline & Child & 30.8 & 47.1 & .343 \\
\hline & Baseline & Parent & 24.0 & 66.7 & $.018^{*}$ \\
\hline & Follow-up & Child & 23.1 & 60.0 & .067 \\
\hline & Follow-up & Parent & 41.7 & 60.0 & .449 \\
\hline \multirow{4}{*}{$\begin{array}{l}\text { Too much traffic } \\
\text { around home }\end{array}$} & Baseline & Child & 15.4 & 41.1 & .080 \\
\hline & Baseline & Parent & 16.0 & 46.7 & $.035^{*}$ \\
\hline & Follow-up & Child & 15.4 & 20.0 & 1.000 \\
\hline & Follow-up & Parent & 41.7 & 20.0 & .398 \\
\hline \multirow{4}{*}{$\begin{array}{l}\text { Too much traffic } \\
\text { around the } \\
\text { school }\end{array}$} & Baseline & Child & 11.5 & 47.1 & $.014^{*}$ \\
\hline & Baseline & Parent & 28.0 & 53.3 & .177 \\
\hline & Follow-up & Child & 0.0 & 33.3 & $.044^{*}$ \\
\hline & Follow-up & Parent & 54.5 & 46.7 & 1.000 \\
\hline \multirow{4}{*}{$\begin{array}{l}\text { Dangerous } \\
\text { crossings }\end{array}$} & Baseline & Child & 19.2 & 37.5 & .281 \\
\hline & Baseline & Parent & 36.0 & 66.7 & .102 \\
\hline & Follow-up & Child & 7.7 & 60.0 & $.006^{*}$ \\
\hline & Follow-up & Parent & 33.3 & 46.7 & .484 \\
\hline \multirow{4}{*}{$\begin{array}{l}\text { Get too hot and } \\
\text { sweaty }\end{array}$} & Baseline & Child & 11.5 & 23.5 & .407 \\
\hline & Baseline & Parent & 0.0 & 26.7 & $.015^{\star}$ \\
\hline & Follow-up & Child & 7.7 & 13.3 & 1.000 \\
\hline & Follow-up & Parent & 0.0 & 13.3 & .487 \\
\hline
\end{tabular}

Note: The percentage of individuals who strongly or somewhat agreed with the barriers statements in the $1^{\text {st }}$ column was compared between active travelers (AT) and inactive travelers (IT) with Fisher's exact test. ${ }^{*}$ Indicates significant differences $(p<.05)$. 


\begin{tabular}{|c|c|c|c|c|c|}
\hline Barriers & $\begin{array}{l}\text { Study } \\
\text { period }\end{array}$ & Respondent & AT $(\%)$ & IT $(\%)$ & $p$ \\
\hline \multirow{4}{*}{$\begin{array}{l}\text { No other children } \\
\text { walk or bike to } \\
\text { school }\end{array}$} & Baseline & Child & 3.8 & 6.3 & 1.000 \\
\hline & Baseline & Parent & 0.0 & 20.0 & $.046^{*}$ \\
\hline & Follow-up & Child & 0.0 & 13.3 & .484 \\
\hline & Follow-up & Parent & 0.0 & 13.3 & .492 \\
\hline \multirow{4}{*}{$\begin{array}{c}\text { Not considered } \\
\text { cool to walk or } \\
\text { bike }\end{array}$} & Baseline & Child & 0.0 & 0.0 & $\mathrm{~N} / \mathrm{A}$ \\
\hline & Baseline & Parent & 0.0 & 13.3 & .135 \\
\hline & Follow-up & Child & 0.0 & 0.0 & $\mathrm{~N} / \mathrm{A}$ \\
\hline & Follow-up & Parent & 0.0 & 6.7 & 1.000 \\
\hline \multirow{4}{*}{$\begin{array}{l}\text { Too much stuff to } \\
\text { carry }\end{array}$} & Baseline & Child & 7.7 & 37.5 & $.038^{*}$ \\
\hline & Baseline & Parent & 4.0 & 42.9 & $.005^{*}$ \\
\hline & Follow-up & Child & 0.0 & 40.0 & $.018^{*}$ \\
\hline & Follow-up & Parent & 8.3 & 33.3 & .182 \\
\hline \multirow{4}{*}{ Easier to drive } & Baseline & Child & 19.2 & 44.4 & .098 \\
\hline & Baseline & Parent & 16.0 & 42.9 & .124 \\
\hline & Follow-up & Child & 7.7 & 46.7 & $.038^{*}$ \\
\hline & Follow-up & Parent & 0.0 & 26.7 & .106 \\
\hline \multirow{4}{*}{$\begin{array}{l}\text { Involves too } \\
\text { much planning } \\
\text { ahead }\end{array}$} & Baseline & Child & 3.8 & 11.8 & .552 \\
\hline & Baseline & Parent & 8.3 & 20.0 & .354 \\
\hline & Follow-up & Child & 7.7 & 26.7 & .333 \\
\hline & Follow-up & Parent & 0.0 & 20.0 & .231 \\
\hline \multirow{4}{*}{$\begin{array}{l}\text { Unsafe because } \\
\text { of crime }\end{array}$} & Baseline & Child & 7.7 & 29.4 & .093 \\
\hline & Baseline & Parent & 0.0 & 26.7 & $.015^{*}$ \\
\hline & Follow-up & Child & 15.4 & 13.3 & 1.000 \\
\hline & Follow-up & Parent & 0.0 & 26.7 & .106 \\
\hline \multirow{4}{*}{ Get bullied } & Baseline & Child & 3.8 & 5.9 & 1.000 \\
\hline & Baseline & Parent & 0.0 & 6.7 & .375 \\
\hline & Follow-up & Child & 7.7 & 6.7 & 1.000 \\
\hline & Follow-up & Parent & 0.0 & 6.7 & 1.000 \\
\hline \multirow{4}{*}{$\begin{array}{c}\text { Nowhere to leave } \\
\text { a bike safely }\end{array}$} & Baseline & Child & 3.8 & 17.6 & .284 \\
\hline & Baseline & Parent & 0.0 & 0.0 & N/A \\
\hline & Follow-up & Child & 7.7 & 33.3 & .173 \\
\hline & Follow-up & Parent & 0.0 & 7.1 & 1.000 \\
\hline \multirow{4}{*}{$\begin{array}{c}\text { There are stray } \\
\text { dogs }\end{array}$} & Baseline & Child & 4.0 & 5.9 & 1.000 \\
\hline & Baseline & Parent & 0.0 & 0.0 & $\mathrm{~N} / \mathrm{A}$ \\
\hline & Follow-up & Child & 7.7 & 6.7 & 1.000 \\
\hline & Follow-up & Parent & 0.0 & 6.7 & 1.000 \\
\hline \multirow{4}{*}{ It's too far } & Baseline & Child & 0.0 & 76.5 & $<.001^{*}$ \\
\hline & Baseline & Parent & 0.0 & 66.7 & $<.001^{*}$ \\
\hline & Follow-up & Child & 7.7 & 66.7 & $<.001^{*}$ \\
\hline & Follow-up & Parent & 0.0 & 53.3 & $.003^{*}$ \\
\hline
\end{tabular}


Table 4. Associations between parental neighborhood selection factors and child's travel mode at baseline and follow-up

\begin{tabular}{|c|c|c|c|c|}
\hline Neighborhood selection factors & $\begin{array}{l}\text { Study } \\
\text { period }\end{array}$ & $\begin{array}{l}\text { AT } \\
(\%)\end{array}$ & IT (\%) & $p$ \\
\hline \multirow{2}{*}{$\begin{array}{c}\text { We liked some aspects of its built } \\
\text { environment (street layout, the } \\
\text { organization of buildings along the } \\
\text { streets, the green spaces \& parks, } \\
\text { etc.) }\end{array}$} & Baseline & 92.0 & 81.3 & .362 \\
\hline & Follow-up & 91.7 & 92.9 & 1.000 \\
\hline \multirow{2}{*}{$\begin{array}{l}\text { We like some aspects of its social } \\
\text { environment, such as proximity to } \\
\text { friends and family }\end{array}$} & Baseline & 52.0 & 68.8 & .344 \\
\hline & Follow-up & 66.7 & 71.4 & 1.000 \\
\hline \multirow{2}{*}{$\begin{array}{l}\text { It was one of the few neighborhoods } \\
\text { we could afford to live in }\end{array}$} & Baseline & 26.1 & 18.8 & .711 \\
\hline & Follow-up & 16.7 & 28.6 & .652 \\
\hline \multirow{2}{*}{$\begin{array}{l}\text { It is easy for our child(ren) to walk or } \\
\text { cycle to school }\end{array}$} & Baseline & 64.0 & 12.5 & $.001^{*}$ \\
\hline & Follow-up & 75.0 & 28.6 & $.047^{*}$ \\
\hline \multirow{2}{*}{$\begin{array}{l}\text { It is a convenient location with } \\
\text { respect to work }\end{array}$} & Baseline & 66.7 & 75.0 & .729 \\
\hline & Follow-up & 83.3 & 64.3 & .391 \\
\hline \multirow{2}{*}{$\begin{array}{l}\text { It is a convenient location with } \\
\text { respect to our household's non-work } \\
\text { activities, such as shopping, } \\
\text { exercise, parks, etc. }\end{array}$} & Baseline & 84.0 & 68.8 & .276 \\
\hline & Follow-up & 91.7 & 64.3 & 170 \\
\hline \multirow{2}{*}{$\begin{array}{l}\text { There are shops and restaurants you } \\
\text { can walk or cycle to }\end{array}$} & Baseline & 64.0 & 43.8 & .334 \\
\hline & Follow-up & 91.7 & 50.0 & $.036^{*}$ \\
\hline
\end{tabular}

Note: all statements began with "We have chosen to live in this neighborhood because". The percentage of individuals who strongly or somewhat agreed with the statements in the $1^{\text {st }}$ column was compared between active travelers (AT) and inactive travelers (IT) with Fisher's exact test. * Indicates significant differences $(p<.05)$. 
Table 5. Associations between socio-demographic characteristics of the household and children's travel mode at baseline and follow-up

\begin{tabular}{|c|c|c|c|c|c|}
\hline Characteristic & Categories & $\begin{array}{l}\text { Study } \\
\text { Period }\end{array}$ & AT $(\%)$ & IT (\%) & $p$ \\
\hline \multirow{2}{*}{ Child gender } & \multirow{2}{*}{$\begin{array}{l}\text { Girls vs. } \\
\text { boys }\end{array}$} & Baseline & 51.9 & 47.4 & 1.000 \\
\hline & & Follow-up & 1.5 & 46.7 & .476 \\
\hline \multirow{2}{*}{$\begin{array}{c}\text { School } \\
\text { neighborhood }\end{array}$} & \multirow{2}{*}{$\begin{array}{l}\text { Higher vs. } \\
\text { lower } \\
\text { density }\end{array}$} & Baseline & 66.6 & 21.1 & $.003^{*}$ \\
\hline & & Fol & 84.6 & 6.7 & $<0.001^{*}$ \\
\hline \multirow{2}{*}{$\begin{array}{l}\text { Number of cars } \\
\text { in the household }\end{array}$} & \multirow{2}{*}{$\leq 1$ vs $\geq 2$} & Baseline & 46.1 & 43.8 & 1.000 \\
\hline & & Follow-up & 69.2 & 14.3 & $0.006^{*}$ \\
\hline \multirow{2}{*}{ Family type } & \multirow{2}{*}{$\begin{array}{l}2 \text { parents } \\
\text { vs. others }\end{array}$} & Baseline & 88.5 & 81.3 & .658 \\
\hline & & Follow-up & 92.3 & 93.3 & 1.000 \\
\hline \multirow{2}{*}{ Family size } & \multirow{2}{*}{$\begin{array}{l}1 \text { children } \\
\text { vs. others }\end{array}$} & Baseline & 11.5 & 18.8 & .658 \\
\hline & & Follow-up & 15.4 & 13.3 & 1.000 \\
\hline \multirow{2}{*}{$\begin{array}{c}\text { Father's } \\
\text { education }\end{array}$} & \multirow{2}{*}{$\begin{array}{l}\text { University } \\
\text { vs. others }\end{array}$} & Baseline & 73.1 & 56.3 & .322 \\
\hline & & Follow-up & 91.7 & 66.7 & .182 \\
\hline \multirow{2}{*}{$\begin{array}{l}\text { Mother's } \\
\text { education }\end{array}$} & \multirow{2}{*}{$\begin{array}{l}\text { University } \\
\text { vs. others }\end{array}$} & Baseline & 84.0 & 68.8 & .276 \\
\hline & & Follow-up & 100.0 & 86.7 & .484 \\
\hline \multirow{2}{*}{$\begin{array}{l}\text { Father's } \\
\text { employment }\end{array}$} & \multirow{2}{*}{$\begin{array}{c}\text { Full time vs. } \\
\text { others }\end{array}$} & Baseline & 96.2 & 86.7 & .543 \\
\hline & & Follow-up & 100.0 & 93.3 & 1.000 \\
\hline \multirow{2}{*}{$\begin{array}{l}\text { Mother's } \\
\text { employment }\end{array}$} & \multirow{2}{*}{$\begin{array}{c}\text { Full time vs. } \\
\text { others }\end{array}$} & Baseline & 69.6 & 56.3 & .503 \\
\hline & & Follow-up & 61.5 & 71.4 & .695 \\
\hline
\end{tabular}

The percentage of individuals with the referent categories mentioned in the $2^{\text {nd }}$ column was compared between active travelers (AT) and inactive travelers (IT) with Fisher's exact test (e.g. at baseline, $51.9 \%$ of active travelers were girls; implying that the remaining $48.1 \%$ were boys). ${ }^{*}$ Indicates significant differences $(p<.05)$.

\section{DISCUSSION}

The present study aimed to compare child- and parent-perceived barriers to AST and to identify how perceived barriers to AST, parental neighborhood selection factors and socio-demographic characteristics of the households relate to children's primary travel mode for the trip to/from school.

Concordance of child- and parent-perceived barriers to AST. In general, children's and parents' perceptions of barriers to AST were significantly correlated at baseline. Using the same instrument, Forman et al. ${ }^{29}$ reported high intraclass correlation coefficients between adolescent and parent reports, but they combined survey items in three subscales instead of examining agreement for each barrier separately. Another study in the US found good agreement between children and parent reports, except within less walkable neighborhoods where parents expressed greater concerns. ${ }^{16}$

However, at follow-up significant correlations were found for only 4 of 19 questionnaire items. Because follow-up data collection occured only a few weeks after participants started secondary school, it is possible that children and/or their parents were less familiar with the route to/from school and the school surroundings. This novelty aspect may have biased the comparisons toward the null hypothesis. Nevertheless, at both time points, the concordance was generally higher for the 
distance and road safety components and most of these components were associated with children's travel mode. To our knowledge, no other study has assessed how the concordance between child- and parent-perceived barriers changes from primary to secondary school, underscoring a need for future research on this topic.

Associations between perceived barriers and AST. In the present study, the strongest perceived barrier to AST was the distance between home and school as perceived by both children and parents. Moreover, in the sub-sample of participants who provided travel mode data at both time points, the average distance increased upon the transition from primary to secondary school while the mode share of AST decreased significantly (R. Larouche, University of Ottawa, unpublished manuscript). These longitudinal findings are consistent with cross-sectional studies comparing the mode share of AST between primary, middle school and high school students in Canada $^{24,32}$ and the United States ${ }^{20}, 23$. In addition, data from the US National Household Travel Survey suggests that a $10 \%$ increase in distance is associated with a $7.5 \%$ decrease in the mode share of walking. ${ }^{10}$ Finally, a systematic review of the relationship between objectively-measured environmental factors and AST found that distance was the only consistent correlate. ${ }^{12}$

Road safety concerns related to heavy traffic were important correlates of travel mode, especially at baseline. However, their influence was not as strong as that of distance, likely because some participants were engaging in AST in spite of their parents' and their own safety concerns. Interestingly, the proportion of children and parents who perceived the absence of sidewalks and cycle paths as a barrier was consistently greater among the inactive travelers, but this relationship was only significant at follow-up among parents. This might be an artifact of the small sample size. In the published literature, several studies have found that perceived road safety was an important barrier to AST. ${ }^{33,}{ }^{36}$ In addition, an Australian study that examined the predictors of increasing AST found that when parents perceived that there was not enough traffic lights and pedestrian crossings in their neighborhood, children were half as likely to begin walking or cycling to school. ${ }^{37}$ Nevertheless, conflicting findings have also been reported with respect to the association between road safety elements and AST. ${ }^{14,38}$

The perception of having too much stuff to carry was associated with inactive travel at baseline and follow-up. This element was mentioned as an important barrier in a qualitative study ${ }^{39}$, although it has not been assessed often in quantitative studies. Technology could help to reduce backpack loads (i.e. using USB sticks and tablets instead of heavy textbooks). Another possibility would be to equip bikes with racks and panniers to avoid the need for carrying heavy loads on one's back. ${ }^{40}$

At baseline only, parents' crime-related concerns were associated with motorized travel; however, there were no associations between children's personal safety concerns and travel mode at any time point. The lack of concordance in this regard warrants future investigation. Nevertheless, it is noteworthy that a literature review has reported inconsistent evidence regarding the relationship between personal safety aspects (e.g. "stranger danger", crime, bullying) and AST among both children and parents. ${ }^{9}$ Given the higher rates of AST among children living in low SES neighborhoods (which also tend to have higher crime rates), SES might be an important confounding variable. ${ }^{41,42}$ 
In the present study, a greater proportion of inactive travelers and their parents reported that "it is easier to drive" and that walking or biking to school "involves too much planning ahead" at both time points. However, the associations were not significant except among children at follow-up. This may reflect the increased distance (and associated time cost) between home and school. Previous research has shown that perceived convenience of driving and time constraints were key reasons underlying parents' decision to drive their children to/from school. ${ }^{43,}{ }^{44}$ Based on semi-structured interviews, Faulkner and colleagues ${ }^{43}$ concluded that parental travel mode decisions are based on two key questions: 1) does the child need to be escorted to/from school?; and 2) what is the easiest and most convenient way to travel? Again, the small sample size may explain why such associations were not found in the present study.

In her theoretical framework. McMillan ${ }^{8}$ assumed that parents are the key decision-makers regarding their children's mode of transport. However, more recent frameworks posit that as children become older, they likely have a greater input into this decision. $^{9,} 13$ This assumption is consistent with the observation that independent mobility (e.g. the degree of freedom of children and youth to move around in public spaces without adult supervision) increases with age. ${ }^{45}$ In turn, independent mobility is associated with greater AST and PA levels. ${ }^{46,}{ }^{47}$ Another study has shown that Canadian adolescents who have some input in the decision making process are more likely to engage in $\mathrm{AST}^{48}$, and an American study indicated that although children- and parent-perceived barriers were correlated, they were still independently associated with the frequency of walking trips. ${ }^{16}$ Together, this body of evidence emphasizes the need to consider both child- and parent-perceived barriers to AST. Similarly, future research should examine how these perceived barriers change over time among both parents and children to inform the development of more effective AST interventions.

Parental neighborhood selection factors. At both time points, children were significantly more likely to engage in AST when their parent reported that they chose to live in their neighborhood so that their children could easily walk or cycle to school. Another study in the Greater Toronto Area found that children were less likely to be driven to school if their parents reported that they had chosen to live in their neighborhood so that their children can walk to school. ${ }^{19}$ In addition, children were more likely to engage in AST at follow-up if their parents chose their neighborhood for the proximity of parks and restaurants. Perceived proximity to such destinations has been shown to be associated with higher physical activity levels in children and youth. ${ }^{49}$ Our findings provide preliminary evidence suggesting that parental neighborhood selection factors can influence thier child's travel modes. Future studies could use a design similar to Frank et al. ${ }^{17}$ to attempt to disentangle the relative influence of self-selection and built environment characteristics.

Socio-demographic characteristics. At both time points, children who attended primary schools located in denser census tracts were more likely to engage in AST. These schools were located significantly closer to participants' home, presumably facilitating AST. Similarly, McDonald ${ }^{8}$ reported that in denser areas, it is more likely that a greater proportion of children live within a walkable distance, suggesting that the effect of density on travel mode may be partially mediated by distance. Nevertheless, there is conflicting evidence with respect to the association between density and AST in the literature. ${ }^{12}$ This may be due to inconsistencies in methodology ${ }^{12}$ to the 
indirectedness of the effect of density (e.g. through distance ${ }^{8}$ ), to a ceiling effect (e.g. beyond a certain level, increasing density may have no effect on AST) or because denser areas may be associated with greater traffic exposure and safety concerns ${ }^{50}$. Density may be less salient when a large proportion of children do not attend a school within their neighborhood as seems to be the case for the participants who attended primary schools in lower density areas. Moreover, differences in AST between school neighborhoods could also be due to other characteristics of the built environment and to school policies that may favor AST to a greater extent in these schools.

Parent's car ownership was the only other socio-demographic variable that was associated with children's travel mode, but this association was found only at follow-up. Specifically, children were almost five times less likely to engage in AST when their parents owned at least two cars. However, only two families did not own a car so they were combined with those that owned one car in analyses. Moreover, children who traveled by car and bus were combined in the analyses; this has likely biased the results toward the null hypothesis. This could explain why our findings differ from those of DiGuiseppi et al. ${ }^{25}$ who reported that British children were much more likely to be driven to school by their parents if they owned at least one car, and the odds were even greater when parents owned two cars. Presumably, higher car ownership makes the option of driving children to/from school more feasible.

The lack of association for the other socio-demographic variables might be due to the small sample size and to the limited variability of these characteristics in the sample (e.g. the majority of parents had university education and almost all fathers worked full time). Therefore, these findings should be interpreted cautiously. North American studies have generally found higher rates of AST in children living in low SES households ${ }^{21,23,24,41}$, in single-parent families ${ }^{24}$, and in boys ${ }^{20-22}$.

Policy implications. The present findings have some implications for policies related to AST. In the US, the school siting guidelines recommend that schools be built on large lots; for example one acre of land for every 100 students plus an additional 10 acres for elementary schools, 20 acres for middle schools and 30 acres for high schools. ${ }^{51}$ Application of these guidelines has led to the construction of large schools in the outskirts of cities; thereby leading to large distances between home and school, especially for high school students. ${ }^{27}$ In the present study, the transition to secondary school was associated with a significant increase in distance and a marked decrease in the mode share of AST. Based on data from the National Household Travel Survey, McDonald ${ }^{8}$ estimated that only $20 \%$ of US students lived within $1.6 \mathrm{~km}$ (1 mile) from their school and argued that this situation greatly reduces the potential effectiveness of Safe Routes to School interventions. To address this issue, a recent policy statement from the American Academy of Pediatrics ${ }^{52}$ recommended that consideration be given to children's ability to engage in AST in the process of determining the location of new schools.

Policies can also be implemented to address some of the road safety barriers identified in the present study and in other investigations. For example, walking school buses can reduce parental safety concerns and foster AST and physical activity. ${ }^{53,54} \mathrm{US}$ schools located in states where crossing guards are required and in school districts with strong Safe Routes to School policies were more likely to implement walking school buses. $^{55}$ 
In another US study, investigators asked the principals of over 600 schools to estimate the proportion of students who engaged in AST and collected information on state transportation laws. ${ }^{56}$ They found that more children engaged in AST in states that required the presence of crossing guards and lower speed limits around schools. In London (UK), the implementation of $20 \mathrm{mph}(32 \mathrm{~km} / \mathrm{h}$ ) speed limits has led to a $49 \%$ decrease in road casualties among children. ${ }^{57}$ In addition, an evaluation of the California Safe Routes to School legislation has shown that urban form changes such as adding or improving sidewalks, traffic lights, pedestrian crossings and bike paths can lead to an increase in AST. ${ }^{58}$ In New York City, a 44\% decrease in pedestrian injuries among school-aged children was observed in census tracts where Safe Routes to School interventions had been implemented while no changes were observed in control census tracts. $^{59}$

Limitations and strengths. The small sample size should be considered when interpreting the present findings for the following reasons. First, statistical power was small, potentially reducing the likelihood of observing significant associations; thus caution is needed in making a distinction between a lack of evidence of effect and evidence of no effect. Second, it was unfeasible to take advantage of the longitudinal study design by examining how changes in perceived barriers are related to changes in travel modes because of the even smaller number of participants who changed travel mode. Future studies should address this research gap to inform the development and refinement of AST interventions. Third, although walking and cycling may have distinct correlates, it was unfeasible to assess the correlates separately. Fourth, analyses were not adjusted for gender and other potential confounders (i.e. school, SES, car ownership). Such variables could moderate (or mediate) the relationship between perceived barriers and children's AST. ${ }^{9}$ However, the mode share of AST did not differ between boys and girls in the present study. Finally, the low participation rate and the large proportion of parents who were university graduates suggest that the sample may not be representative of the Canadian population. Approval from school principals was mandatory before proceeding with data collection, and all primary schools that granted approval were included in the study. This may have led to a greater participation of schools located in higher SES neighborhoods. The longitudinal study design, and the school board requirement that incentives can not be offered to participants, may have contributed to the low participation rate.

Nevertheless, the present study has important strengths. Of particular interest, the association between children's travel mode and perceived barriers to AST was examined at the end of primary school and the beginning of secondary school among the same group of participants. Second, no other study has systematically compared the concordance between child- and parent-perceived barriers to AST before and after the school transition. Third, a wide variety of potential correlates of AST were considered, many of which have not been studied extensively. Furthermore, while several prospective studies have shown large decreases in physical activity levels from childhood to adolescence ${ }^{26}$, little is known about the influence of the school transition on AST. Only two studies have examined this question longitudinally ${ }^{60}(R$. Larouche, University of Ottawa, unpublished manuscript) and both reported a decrease in the mode share of AST. 


\section{CONCLUSION}

To our knowledge, this pilot-study is the first investigation to examine the correlates of AST immediately before and after the transition from primary to secondary school within the same group of participants. The school transition might be an important period for promoting AST in order to attenuate the age-related decline in physical activity. Significant concordance was found between child- and parentperceived barriers to AST at baseline, but not at follow-up; this might be due to a novelty effect with respect to the route and the secondary school environment. In line with other studies, distance between home and school consistently emerged as the strongest barrier to AST. Road safety issues and the perception of having too much stuff to carry were other important barriers to AST. Children were also more likely to engage in AST when their parents reported that they chose their neighbourhood so that their children could easily walk or bike to school, indicating that future studies should take parental neighborhood selection into account. Together, the present findings should be useful in stimulating future research on this topic as well as informing the development (and refinement) of policy and program interventions to promote AST in primary and secondary schools. 


\section{REFERENCES}

1. de Nazelle A, Nieuwenhuijsen MJ, Anto JM, et al. Improving health through policies that promote active travel: A review of evidence to support health impact assessment. Environ Int. 2011;37(4):766-777.

2. Larouche $R$. The environmental and population health benefits of active transport: A review. In: Liu G (Ed.) Greenhouse Gases - Emissions, Measurement and Management. Rijeka, Croatia: InTech; 2012:313-340.

3. Sallis JF, Cervero RB, Ascher W, Henderson KA, Kraft MK, Kerr J. An ecological approach to creating active living communities. Annu Rev Public Health, 2006;27:297-322.

4. van der Ploeg HP, Merom D, Corpuz G, Bauman AE. Trends in Australian children traveling to school 1971-2003: burning petrol or carbohydrates? Prev Med. 2008;46(1):60-62.

5. Buliung RN, Mitra R, Faulkner G. Active school transportation in the Greater Toronto area, Canada: an exploration of trends in space and time (1986-2006). Prev Med. 2009;48(6):507-512.

6. McDonald NC. Active commuting to school: trends among US schoolchildren 1969-2001. Am J Prev Med. 2007;32(6):509-516.

7. Chillon P, Evenson KR, Vaughn A, Ward DS. A systematic review of interventions for promoting active transportation to school. Int J Behav Nutr Phys Act. 2011;8:10.

8. McMillan TE. Urban form and a child's trip to school: the current literature and a framework for future research. J Planning Literature. 2005;19(4):440-456.

9. Panter JR, Jones AP, van Sluijs EMF. Environmental determinants of active travel in youth: a review and framework for future research. Int $J$ Behav Nutr Phys Act. 2008;5:34.

10. McDonald NC. Children's mode choice for the school trip: the role of distance and school location in walking to school. Transportation. 2008;35:23-35.

11. Nelson NM, Foley E, O'Gorman DJ, Moyna NM, Woods CB. Active commuting to school: how far is too far? Int J Behav Nutr Phys Act. 2008;5(1).

12. Wong BYM, Faulkner G, Buliung R. GIS measured environmental correlates of active school transport: A systematic review of 14 studies. Int J Behav Nutr Phys Act. 2011;8(39).

13. Pont K, Ziviani J, Wadley D, Abbott R. The model of children's active travel (MCAT): A conceptual framework for examining factors influencing children's active travel. Aust Occup Ther J. 2011;58:138-144.

14. Timperio A, Crawford D, Telford A, Salmon J. Perceptions about the local neighborhood and walking and cycling among children. Prev Med. 2004;38(1):39-47.

15. Timperio A, Ball K, Salmon J, et al. Personal, family, social, and environmental correlates of active commuting to school. Am J Prev Med. 2006;30(1):45-51.

16. Napier MA, Brown BB, Werner CM, Gallimore J. Walking to school: community design and child and parent barriers. J Environ Psychol. 2011;31(1):45-51.

17. Frank LD, Saelens BE, Powell KE, Chapman JE. Stepping towards causation: do built environments or neighborhood and travel preferences explain physical activity, driving, and obesity? Soc Sci Med. 2007;65:1898-1914. 
18. Handy S, Cao X, Mokhtarian P. Self-selection in the relationship between the built environment and walking: Empirical evidence from Northern California. J Am Plan Assoc. 2006;72(1): 55-74.

19. Mammen G, Faulkner G, Buliung R, Lay J. Understanding the drive to escort: a cross-sectional analysis examining parental attitudes toward children's school travel and independent mobility. BMC Public Health. 2012;12:862.

20. Fulton JE, Shisler JL, Yore MM, Caspersen CJ. Active transportation to school: Findings from a national survey. Res Q Exerc Sport. 2005;76(3):352-357.

21. Gropp K, Pickett W, Janssen I. Multi-level examination of correlates of active transportation to school among youth living within 1 mile of their school. Int $J$ Behav Nutr Phys Act. 2012;9,124.

22. McMillan T, Day K, Boarnet M, Alfonzo M, Anderson C. Johnny walks to school does Jane? Sex differences in children's active travel to school. Child Youth Environ. 2006;16(1):75-89.

23. Evenson KR, Huston SL, McMillen BJ, Bors P, Ward DS. Statewide prevalence and correlates of walking and bicycling to school. Arch Pediatr Adolesc Med. 2003;157(9):887-892.

24. Pabayo R, Gauvin L, Barnett TA. Longitudinal changes in active transportation to school in Canadian children aged 6 through 16 years. Pediatrics. 2011;128(2):e404-413.

25. DiGuiseppi C, Roberts I, Li L, Allen, D. Determinants of car travel on daily journeys to school: cross sectional survey of primary school children. BMJ. 1998;316:1426-1428.

26. Dumith SC, Gigante DP, Domingues MR, Kohl III HW. Physical activity change during adolescence: a systematic review and a pooled analysis. Int $\mathrm{J}$ Epidemiol. 2011;40:685-698.

27. Environmental Protection Agency. Travel and environmental implications of school siting. Washington, DC: Environmental Protection Agency; 2003.

28. Statistics Canada. Census tract profiles, 2006 Census. Ottawa, ON: Statistics Canada. http://www12.statcan.ca/census-recensement/2006/dp-pd/prof/92597/index.cfm?Lang=E Accessed January 29, 2013.

29. Forman H, Kerr J, Norman GJ, et al. Reliability and validity of destination-specific barriers to walking and cycling. Prev Med. 2008;46(4):311-316.

30. Fisher RA. On the interpretation of $X^{2}$ from contingency tables, and the calculation of P. J Royal Stat Soc. 1922;85(1):87-94.

31. Statistics Canada. Educational attainment and employment: Canada in an international context. Ottawa, ON: Statistics Canada; http://www.statcan.gc.ca/pub/81-599-x/81-599-x2012008-eng.pdf Accessed January 31, 2013.

32. Pabayo R, Gauvin L. Proportion of students who use various modes of transportation to and from school in a representative population-based sample of children and adolescents, 1999. Prev Med. 2008;46(1):63-66.

33. Carver A, Timperio A, Crawford D. Playing it safe: the influence of neighbourhood safety on children's physical activity (a review). Health Place. 2008;14(2):217-227. 
34. Kerr J, Rosenberg D, Sallis JF, Saelens BE, Frank LD, Conway TL. Active commuting to school: associations with environment and parental concerns. Med Sci Sports Exerc. 2006;38(4):787-794.

35. Martin S, Carlson S. Barriers to children walking to or from school - United States, 2004. Morb Mortal Wkly Rep. 2005;54:949-952.

36. Voorhees CC, Ashwood S, Evenson KR, et al. Neighborhood design and perceptions: Relationship with active commuting. Med Sci Sports Exerc. 2010;41(7):1253-1260.

37. Hume C, Timperio A, Salmon J, et al. Walking and cycling to school: Predictors of increase among children and adolescents. Am J Prev Med. 2009;36(3):195200.

38. Carson V, Kuhle S, Spence JC, Veugelers PJ. Parent's perception of neighborhood environment as a determinant of screen time, physical activity and active transport. Can J Public Health. 2010;101(2):124-127.

39. Ahlport KN, Linnan L, Vaughn A, Evenson KR, Ward DS. Barriers and facilitators of walking and bicycling to school: formative results from the Non-motorized Travel Study. Health Educ Behav. 2008;35(2):221-244.

40. Lovejoy K, Handy S. Developments in bicycle equipment and its role in promoting cycling as a travel mode. In J. Pucher and R. Buehler (eds.) City cycling (pp. 75-104.). Cambridge, MA: Massachusetts Institute of Technology Press; 2012.

41. Pabayo R, Gauvin L, Barnett TA, Morency $P$, Nikiéma $B$, Séguin, L. Understanding the determinants of active transportation to school among children: evidence of environmental injustice from the Quebec Longitudinal Study of Child Development. Health Place, 2012;18(2):163-171.

42. Rossen LM, Pollack KM, Curriero FC, et al. Neighborhood incivilities, perceived neighborhood safety, and walking to school among urban-dwelling children. $J$ Phys Act Health. 2011;8(2):262-271.

43. Faulkner GEJ, Richichi V, Buliung RN, Fusco C, Moola F. What's "quickest and easiest?": parental decision making about school trip mode. Int J Behav Nutr Phys Act. 2010;7(62).

44. McDonald NC, Aalborg AE. Why parents drive children to school: Implications for Safe Routes to School programs. J Am Plan Assoc. 2009;75(3):331-342.

45. Hillman M, Adams J, Whitelegg J. One false move: A study of children's independent mobility. London: PSI Publishing; 1990.

46. Page A, Cooper AR, Griew P, Jago R. Independent mobility, perceptions of the built environment, and children's participation in play, active travel and structured exercise and sport: the PEACH project. Int J Behav Nutr Phys Act. 2010;7(1).

47. Schoeppe S., Duncan MJ, Badland H, Oliver M, Curtis C. Associations of children's independent mobility and active travel with physical activity, sedentary behaviour and weight status: a systematic review. J Sci Med Sport. In press.

48. Wong BYM, Faulkner G, Buliung R, Irving, H. Mode shifting in school travel mode: examining the prevalence and correlates of active school transport in Ontario, Canada. BMC Public Health, 11, 618. 
49. Ding DG, Sallis JF, Kerr J, Lee S, Rosenberg DE. Neighborhood environment and physical activity among youth: a review. Am J Prev Med. 2011;41(4):442455.

50. Giles-Corti B, Kelty SF, Zubrick SR, Villanueva KP. Encouraging walking for transport and physical activity in children and adolescents: how important is the built environment? Sports Med. 2009;39(12):995-1009.

51. Council of Educational Facility Planners International. Guide for planning educational facilities. Council of Educational Facility Planners International, Scottsdale, AZ; 1991.

52. American Academy of Pediatrics. The built environment: designing communities to promote physical activity in children. Pediatrics. 2009;123(6):1591-1598.

53. Heelan KA, Abbey BM, Donnelly JE, Mayo MS, Welk GJ. Evaluation of a walking school bus for promoting physical activity in youth. J Phys Act Health. 2009;6(5):560-567.

54. Mendoza JA, Watson K, Baranowski T, Nicklas TA, Uscanga DK, Hanfling MJ. The walking school bus and children's physical activity: a pilot cluster randomized controlled trial. Pediatrics. 2011;128(3):e537-e544.

55. Turner L, Chriqui J, Chaloupka F. Walking school bus programs in U.S. public elementary schools. J Phys Act Health. In press.

56. Chriqui JF, Taber DR, Slater SJ, Turner L, Lowrey KM, Chaloupka FJ. The impact of state safe routes to school-related laws on active travel to school policies and practices in U.S. elementary schools. Health Place. 2012;18(1):8-15.

57. Grundy C, Steinbach R, Edwards P, Green J, Armstrong B, Wilkinson P. (2009) Effect of 20mph traffic speed zones on road injuries in London, 1986-2006: controlled interrupted time series analysis. BMJ, 3369;b4469.

58. Boarnet M, Anderson CL, Day K, McMillan T, Alfonzo M. Evaluation of the California Safe Routes to School legislation: urban form changes and children's active transportation to school. Am J Prev Med. 2005;28(Suppl. 2):134-140.

59. DiMaggio C, Li G. Effectiveness of a Safe Routes to School program in preventing school-aged pedestrian injury. Pediatrics. 2013;131:290-296.

60. Cooper AR, Jago R, Southward EF, Page AS. Active travel and physical activity across the school transition: the PEACH project. Med Sci Sports Exerc. 2012;44:1890-1897. 
Chapter 7. Study VI: “Associations between neighbourhood walkability, active school transport and physical activity levels in primary and secondary school students: a pilotstudy"

Authors: Richard Larouche, Guy Faulkner, Mark S. Tremblay

Status $^{4}$ : Published in the University of Ottawa Journal of Medicine (2013), Vol. 3, p. 42-46

Submitted for presentation at the 2013 Canadian Society for Exercise Physiology Conference.

\footnotetext{
${ }^{4}$ The approved manuscript version is presented here because an error was made in the English abstract by the Editorial team after I have approved the author proofs.
} 
Associations between neighbourhood walkability, active school transport and physical activity levels in primary and secondary school students: a pilot-study

Abstract word count: 234

Manuscript word count: 2414 


\section{ABSTRACT:}

Objectives: This longitudinal pilot-study examined the associations of neighbourhood walkability with active school transport (AST) and pedometer-determined physical activity (PA) immediately before and after the transition from primary to secondary school.

Methods: Fifty-five grade 6 students were recruited from 4 primary schools in Ottawa in May/June 2012. They were asked to complete a diary indicating their mode of transport to/from school for 1 week and wear a SC-StepMX pedometer for 8 consecutive days. 48 study packages were returned at baseline and 29 at follow-up (September/October 2012). The Walk Score ${ }^{\circledR}$ application was used as a proxy for walkability around the home and around the school. The associations of walkability with AST and average daily step counts at both time points were respectively examined with binary logistic regression and linear regression models adjusted for gender.

Results: At baseline, only walkability around the school was positively associated with AST $(\mathrm{OR}=1.04)$. At follow-up, higher Walk Score ratings around the home and the school were both associated with greater odds of AST (OR $=1.12$ and 1.29 respectively). Furthermore, walkability around the home was associated with higher step counts with a large effect size $\left(\eta^{2}=0.19\right)$.

Conclusion: These preliminary findings suggest that walkability may be more important for secondary school students. Because no other longitudinal study has assessed whether the associations of walkability with AST and PA changes over time, larger prospective studies are warranted.

\section{RÉSUMÉ :}


Objectifs : Cette étude pilote longitudinale vérifiait l'association entre les quartiers favorables à la marche, le transport actif scolaire (TAS) et le niveau d'activité physique (AP) déterminé à l'aide d'un podomètre, immédiatement avant et après la transition de l'école primaire à l'école secondaire.

Méthodes : En mai et juin 2012, 55 élèves de la sixième année ont été recrutés dans 4 écoles primaires d'Ottawa. On leur a demandé de remplir quotidiennement un journal de bord dans lequel ils devaient indiquer leur mode de transport pour se rendre à l'école et en revenir durant une semaine. Ils devaient également porter un podomètre SC-StepMX durant huit jours consécutifs. 48 trousses d'étude ont été retournées à la première étape et 29 lors du suivi en septembre et octobre 2012. L'application Walk Score ${ }^{\circledR}$ a été utilisée comme témoin pour déterminer si le milieu environnant le domicile et l'école était favorable à la marche. L'association entre les quartiers favorables à la marche, le TAS et le nombre moyen de pas faits dans une journée a été examinée respectivement aux deux étapes de l'étude à l'aide d'une régression logistique binaire et d'un modèle de régression linéaire ajusté selon le sexe.

Résultats : À l'étape initiale, seul un milieu favorable à la marche autour de l'école était associé positivement au TAS $(\mathrm{RC}=1,04)$. Lors du suivi, un indice Walk Score élevé aux alentours de la maison et de l'école était associé à une probabilité supérieure de TAS $(\mathrm{RC}=1,12$ et 1,29 respectivement). De plus, un quartier favorable à la marche autour du domicile était associé à un nombre plus élevé de pas avec une taille d'effet élevée $\left(\eta^{2}=0,19\right)$.

Conclusion : Ces observations préliminaires suggèrent qu'il peut s'avérer plus important pour les étudiants de l'école secondaire que leur milieu soit favorable à la marche. Puisqu'aucune autre étude longitudinale n'a été menée pour évaluer si l'association entre les quartiers 
favorables à la marche, le TAS et le niveau d'AP varie au fil du temps, il serait justifié de procéder à de grandes études prospectives.

\section{Introduction}

Recent survey findings indicate that the majority of children and youth do not meet current physical activity (PA) guidelines, which recommend 60 minutes of moderate-to-vigorous physical activity per day [1,2]. Even in the pediatric population, insufficient PA levels are associated with detrimental health parameters such as cardiovascular disease risk factors [3,4], underscoring the need for interventions that promote PA. Since children and youth must travel to and from school on a regular basis, active school transport (AST; e.g. using non-motorized travel modes such as walking and cycling to travel to/from school) is regarded as a promising strategy to increase PA at the population level $[5,6]$.

During the last two decades, there has been a rapid increase in the number of scientific studies assessing the association between characteristics of the built environment (i.e. density, land use mix, street connectivity, availability of sidewalks and cycle paths, etc.) and individuals' active transport and PA levels [7]. Researchers have developed composite measures of neighbourhood characteristics that favour "walkability", which refers to the potential for individuals to walk to local destinations $[8,9]$.

Measures of neighbourhood walkability have consistently been shown to be associated with adults' travel mode and PA [10,11]. However, the evidence regarding the association between characteristics of the built environment and children's AST and PA remains inconsistent $[12,13]$. In their systematic review of 14 studies that used objective measures of environmental 
characteristics, Wong et al. found that distance between home and school was the only consistent correlate of AST [13].

Giles-Corti et al. [14] hypothesized that the influence of the built environment on AST and PA may be stronger in adolescents than in children because the former generally have greater independent mobility. This concept refers to the degree of freedom of children and youth to move around in public spaces without adult supervision [15]. To date, most studies that examined the association between the built environment and AST have only considered associations with individual characteristics rather than composite measures of walkability [13]. One US study found that characteristics of the built environment had a stronger association with walking in youth aged 12-15 years old compared to younger participants [16]; however the cross-sectional design is an important limitation of this study.

Therefore, the objective of the present pilot-study was to examine the associations of neighbourhood walkability with AST and PA at the end of primary school (grade 6) and the beginning of secondary school (grade 7) among the same participants. The school transition is a major life event that coincides with a large decrease in PA [17]. It was hypothesized that the associations of neighbourhood walkability with AST and PA would be stronger in secondary school.

\section{Methods}

Participants and setting. Fifty-five grade 6 students were recruited from four primary schools in Ottawa (Canada) in May/June 2012 (33.3\% response rate). Of these four schools, two were located in census tracts with high population density (3531-4100 inhabitants $\left./ \mathrm{km}^{2}\right)$, according to the 2006 Canadian census data [18]. The two other schools were located in lower density areas 
(988-2159 inhabitants $/ \mathrm{km}^{2}$ ), thereby providing variability in built environment characteristics. Parents indicated their child's prospective school for grade 7 and either their phone number or email address for follow-up purposes. 48 children ( 24 girls and 24 boys) returned their study package at baseline and 29 (16 girls and 13 boys) at follow-up (September/October 2012). Ethical approval was obtained from institutional Research Ethics Boards and from the 2 participating school boards.

Measures At both time points, participants were asked to: 1) complete a travel diary indicating their mode of transport to/from school for each day during 1 week; 2) wear a SC-StepMX pedometer (Stepscount, Deep River, ON) on the right hip for 8 consecutive days; and 3) complete a log recording their daily step counts and the time the pedometer was worn during waking hours. This pedometer has been shown to be valid and reliable [19]. Parents of each participant indicated their postal code, which allowed for the estimation of neighbourhood walkability.

Data treatment. Participants were classified as active travelers if they reported using active travel modes for at least $50 \%$ of school trips. This classification method showed very high testretest reliability over two consecutive weeks of measurement [20]. Pedometry data were screened based on established criteria including: 1) between 1,000 and 30,000 steps/day [21]; 2) $\geq 10$ hours of data/day [22] and 3) $\geq 3$ days of valid data (e.g. meeting the daily wear threshold values) [23]. Application of these thresholds led to the exclusion of pedometer data from 2 participants at baseline and none at follow-up. The postal codes provided by the parent were used as a proxy for residential address, based on evidence that Canadian postal codes are a suitable 
proxy in urban areas $[24,25]$. The Walk Score ${ }^{\circledR}$ application (http://www.walkscore.com/) was used as an estimate of neighbourhood walkability around the participants' residence (using postal codes) and around their school (using the street address) at both time points. The Walk Score is a composite measure of accessibility to a variety of destinations including schools, parks, shops, and public transit by walking. Amenities located within a $1.6 \mathrm{~km}$ linear buffer contribute to a location's Walk Score, but amenities within a 400 meters buffer receive higher points than those within 800 meters, $1.2 \mathrm{~km}$ and $1.6 \mathrm{~km}$ buffers. Walk Score ratings range from 0 to 100 with higher values indicating greater walkability. A validation study has found strong correlations between Walk Score ratings and objective measures of the built environment (i.e. residential density, intersection density, street density, average block length and access to public transit) [26].

Analyses. First, Pearson correlations assessed the association between Walk Score ratings around the home and around the school at both time points. Second, the associations of neighbourhood walkability with AST and average daily step counts at both time points were examined with binary logistic regression and linear regression models respectively, and adjusted for gender. The $\eta^{2}$ statistic was used as a measure of the independent effect size of neighbourhood walkability within the regression models. Analyses were performed with IBM SPSS 20 and the probability of type I error was set at 5\%.

\section{Results}

Descriptive characteristics of the participants at baseline and follow-up are provided in Table 1. Baseline data did not differ between participants who provided follow-up data and those 
who provided only baseline data with respect to gender, Walk Score, AST and PA (all $p>0.49$ ). Walk Score ratings around the home and around the school were significantly correlated at baseline $(\mathrm{r}=0.70 ; p<.001)$ and follow-up $(\mathrm{r}=0.53 ; p=.003)$; therefore, to avoid multicollinearity, separate models were done to assess the effect of walkability around the home and around the school.

At baseline, Walk Score ratings around the home did not differ between active and inactive travelers $(\mathrm{OR}=0.99 ; 95 \% \mathrm{CI}=0.96-1.03 ; p=.87)$, but children attending schools with higher ratings were more likely to engage in AST $(\mathrm{OR}=1.04 ; 95 \% \mathrm{CI}=1.01-1.07 ; p=.03)$ (Table 2). At follow-up, higher Walk Score ratings around the home $(\mathrm{OR}=1.12 ; 95 \% \mathrm{CI}=1.03$ $1.21 ; p=.01)$ and around the school $(\mathrm{OR}=1.29 ; 95 \% \mathrm{CI}=1.00-1.66 ; p=.05)$ were both associated with greater odds of AST. Of note, these odds ratios represent the change in the odds of AST associated with each unit increase in Walk Score. For instance, a 10-point increase in Walk Score around the home at follow-up would be associated with a 3 -fold increase $(\mathrm{OR}=$ 3.02) in the odds of AST.

Table 3 illustrates the association between Walk Score and average daily step counts at both time points. At baseline, Walk Score ratings around the home and around the school were not associated with step counts (all $p>.79$ ). At follow-up, children living in more walkable areas were significantly more active $(\mathrm{F}=5.21 ; p=.03)$ with a large effect size $\left(\eta^{2}=0.19\right)$, but no association was found for walkability around the school $(\mathrm{F}=1.97 ; p=.17)$. In all regression models, boys were more active than girls (all $p \leq .04$ ); however, there were no gender differences in travel modes at any time point $(p \geq .62)$.

\section{Discussion}


The present pilot-study assessed the influence of neighbourhood walkability on AST and PA immediately before and after the transition from primary to secondary school, a major life event that has been understudied with respect to AST. At baseline, walkability measures were not associated with PA, and only Walk Score ratings around the school were associated with AST. At follow-up, Walk Score ratings around the home and the school were both strongly associated with AST. Walkability around the home was significantly associated to PA with a large effect size; however, this relationship was not found for walkability around the school. Despite some inconsistencies, these findings provide preliminary evidence supporting GilesCorti and colleagues' [14] hypothesis that the influence of neighbourhood walkability on AST and PA levels is stronger in secondary school students than in primary school students.

Previous systematic reviews have concluded that the evidence supporting associations between neighbourhood walkability (or different built environment constructs), AST and PA is inconsistent and that most included studies were cross-sectional [12,13]. Inconsistent findings could be due to many factors including methodological differences in the assessment of built environment characteristics [13,27] or PA levels [12] and failure to consider potential moderators such as age, gender, ethnicity and socioeconomic status [28,29].

In addition, it has been suggested that the influence of built environment characteristics may be additive $[7,30]$, so studies that examine individual characteristics instead of using a composite measure of walkability may underestimate the strength of observed associations. McDonald [31] reported that the direct influence of population density on AST was weak, but that higher density may lead to shorter distances between home and school, which is in turn strongly associated with AST. Furthermore, there may be complex interactions between barriers 
and facilitators of AST. For example, high density and street connectivity may be associated with both shorter distance to school and heavier traffic exposure [14].

Another potential reason for the lack of association between Walk Score ratings and PA at baseline is that school journeys may account for a lower proportion of daily PA in children than in adolescents [32,33]. Primary school children may accumulate a greater proportion of their PA through active play than secondary school youth. Therefore, low density neighbourhoods with larger lots and cul-de-sacs may be more conducive to active play than high density neighbourhoods with heavy traffic and associated road safety concerns. In contrast, as children get older, active transport (not only to/from school) may become a more important source of PA; hence, walkable neighbourhoods could become increasingly important for fostering PA.

The school transition might be a good time for interventions that promote AST because travel habits are likely to be modified due to changes in school location. In a Scottish study, travel habits explained a significant proportion of the variance in step counts during the trip to school over and above planned behaviour constructs (e.g. attitudes, subjective norms and perceived behavioural control) [34]. In addition, Panter et al. [29] suggested that parental safety concerns might become less influential in travel mode decisions as children acquire independent mobility.

The main limitations of this study are the small sample size and the low response rate suggesting that the sample population may not be representative of the general population. As a result, there may have been a selection bias with highly active children being more likely to participate at baseline. Furthermore, the small sample size precludes the adjustment of regression models for a larger range of socio-demographic variables that may influence travel mode choices 
and PA patterns (i.e. parental education and employment status, car ownership). Children may have increased their level of PA when wearing a pedometer; however, a recent review of pedometer use among children reported conflicting findings regarding the issue of pedometer reactivity [35]. Thus, it remains unclear whether reactivity may have contributed to the observed changes in physical activity. However, the observed changes are consistent with the declines in physical activity commonly seen during this transitional period [17]. Many studies have shown that children are more active during summer months, thus the observed differences in step counts may be partially explained by seasonal variations [36]. In this study, the follow-up was done as early as possible in the school year to minimize seasonality bias. Interestingly, previous research in Toronto (Canada) has shown no seasonal differences in travel modes among 11-12 years old children [37]. The observed decrease in the proportion of participants engaging in AST may be attributable to a $50 \%$ increase in the distance between home and school across the school transition [20]. Although Walk Score ratings have been shown to be correlated with other aspects of the built environment (i.e. density, diversity and design), it may fail to capture characteristics associated with younger children's travel and PA patterns. Finally, postal codes were used as a proxy of residential address as required by Research Ethics Boards. Nevertheless, previous research in Calgary (Canada) has shown that $87.9 \%$ of postal code locations were within 200 meters of the true address location [24].

The main strength of the study is the assessment of neighbourhood walkability, AST and PA immediately before and after the school transition, a major life event usually characterized by a large decrease in PA levels. Moreover, walkability was assessed both around the home and around the school. The use of an objective measure of PA, rather than a questionnaire, is another important strength because using a questionnaire can lead to a large overestimation of children's 
PA level [38]. To our knowledge, only one other study has assessed the influence of the school transition on AST [39], but no associations of built environment characteristics with AST and PA were reported.

\section{Conclusion}

In this longitudinal pilot-study, the association between neighbourhood walkability (as assessed by the Walk Score application) and measures of AST and PA was stronger in secondary school compared to primary school. This suggests that neighbourhood walkability may be more important for supporting adolescents' AST and PA levels. However, given the small sample size, future prospective studies are needed to confirm these findings. Since the school transition has been shown to coincide with a large decrease in PA [17], there is a need for studies to examine whether walkable environments can attenuate this decline.

\section{References}

1. Colley RC, Garriguet D, Janssen I, Craig CL, Clarke J, Tremblay MS. Physical activity of Canadian children and youth: Accelerometer results from the 2007 to 2009 Canadian Health Measures Survey. Health Reports. 2011;22(1):15-24.

2. Hallal P, Andersen LB, Bull FC et al. Physical activity levels of the world population: surveillance progress, gaps and prospects. Lancet. 2012;380(9838):247-57.

3. Andersen LB, Harro M, Sardinha LB et al. Physical activity and clustered cardiovascular risk in children: a cross-sectional study (The European Youth Heart Study). Lancet. 2006;368:299-304. 
4. Ekelund U, Luan J, Sherar LB, Esliger DW, Griew P, Cooper AR. Moderate to vigorous physical activity and sedentary time and cardiometabolic risk factors in children and adolescents. JAMA. 2012;307(7):704-12.

5. Faulkner GEJ, Buliung RN, Flora PK, Fusco C. Active school transport, physical activity levels and body weight of children and youth: a systematic review. Prev Med. 2009;48(1):3-8

6. Larouche R. The environmental and population health benefits of active transport: A review. In: Liu G, ed. Greenhouse Gases - Emissions, Measurement and Management. Rijeka, Croatia: InTech. 2012. 313-40 p.

7. Sallis JF, Floyd MF, Rodriguez DA, Saelens BE. Role of built environments in physical activity, obesity, and cardiovascular disease. Circulation. 2012;125:729-37.

8. Cervero R, Kockelman K. Travel demands and the 3Ds: density, diversity and design. Transp Res Part D. 1997;2(3):199-219.

9. Gauvin L, Richard L, Craig CL et al. From walkability to active living potential: an "ecometric" validation study. Am J Prev Med. 2005;28(Suppl. 2):126-33.

10. Sallis JF, Cervero RB, Ascher W, Henderson KA, Kraft MK, Kerr J. An ecological approach to creating active living communities. Annu Rev Public Health. 2006;27:297322.

11. Saelens BE, Handy S. Built environment correlates of walking: a review. Med Sci Sports Exerc. 2008;40(Suppl. 7):S550-S66.

12. Ding DG, Sallis JF, Kerr J, Lee S, Rosenberg DE. Neighborhood environment and physical activity among youth: a review. Am J Prev Med. 2011;41(4):442-55. 
13. Wong BYM, Faulkner G, Buliung R. GIS measured environmental correlates of active school transport: A systematic review of 14 studies. Int J Behav Nutr Phys Act. $2011 ; 8(39)$.

14. Giles-Corti B, Kelty SF, Zubrick SR, Villanueva KP. Encouraging walking for transport and physical activity in children and adolescents: how important is the built environment? Sports Med. 2009;39(12):995-1009.

15. Hillman M, Adams J, Whitelegg J. One false move: A study of children's independent mobility. London, UK: PSI Publishing; 1990.

16. Frank L, Kerr J, Chapman J, Sallis J. Urban form relationships with walk trip frequency and distance among youth. Am J Health Promot. 2007;21(Suppl. 4):305-11.

17. Dumith SC, Gigante DP, Domingues MR, Kohl III HW. Physical activity change during adolescence: a systematic review and a pooled analysis. Int J Epidemiol. 2011;40:685-98.

18. Statistics Canada. Census tract profiles, 2006 Census [Internet]. Statistics Canada, Ottawa, ON; 2006 [Cited January 29, 2013]. Available from: http://www12.statcan.ca/census-recensement/2006/dp-pd/prof/92597/index.cfm?Lang=E.

19. Colley RC, Barnes JD, Leblanc AG, Borghese M, Boyer C, Tremblay MS. Validity of the SC-StepMX pedometer during treadmill walking and running. Appl Physiol Nutr Metabol. In press.

20. Larouche R, Faulkner G, Tremblay MS. Assessing the influence of the transition from primary to secondary school on the volume of active school transport and physical activity: a prospective pilot-study. Bioenergetics. Submitted. 
21. Rowe D, Mahar M, Raedeke TD, Lore J. Measuring physical activity in children with pedometers: reliability, reactivity, and replacement of missing data. Pediatr Exerc Sci. 2004;16(4):343-54.

22. Colley RC, Connor Gorber S, Tremblay MS. Quality control and data reduction procedures for accelerometry-derived measures of physical activity. Health Reps. 2010;21(1):63-9.

23. Tudor-Locke C, Burkett L, Reis JP, Ainsworth BE, Macera CA, Wilson DK. How many days of pedometer monitoring predict weekly physical activity in adults? Prev Med. 2005;40(3):293-8.

24. Bow CJD, Waters NM, Faris PD et al. Accuracy of city postal code coordinates as a proxy for location of residence. Int J Health Geogr. 2004;3(5).

25. Healy M, Gilliland J. Quantifying the magnitude of environmental exposure misclassification when using imprecise address proxies in public health research. Spat Spatiotemporal Epidemiol. In press.

26. Carr LJ, Dunsiger SI, Marcus BH. Walk Score ${ }^{\mathrm{TM}}$ as a global estimate of neighbourhood walkability. Am J Prev Med. 2010;39(5):460-3.

27. Mitra R, Buliung RN. Built environment correlates of active school transportation: neighbourhood and the modifiable areal unit problem. J Transp Geogr. 2012;20:51-61.

28. Kerr J, Frank L, Sallis JF, Chapman J. Urban form correlates of pedestrian travel in youth: differences by gender, race-ethnicity and household attributes. Transp Res Part D. $2007 ; 12: 177-82$. 
29. Panter JR, Jones AP, van Sluijs EMF. Environmental determinants of active travel in youth: a review and framework for future research. Int $\mathrm{J}$ Behav Nutr Phys Act. 2008;5(34).

30. Sallis JF, Bowles HR, Bauman A, Ainsworth BE, Bull FC, Craig CL. Neighborhood environments and physical activity among adults in 11 countries. Am J Prev Med. 2009;36(6):484-90

31. McDonald NC. Children's mode choice for the school trip: the role of distance and school location in walking to school. Transportation. 2008;35:23-35.

32. Cooper AR, Page AS, Wheeler BW et al. Mapping the walk to school using accelerometry combined with a global positioning system. Am J Prev Med. 2010;38(2):178-83.

33. Southward EF, Page AS, Wheeler BW, Cooper AR. Contribution of the school journey to daily physical activity in children aged 11-12 years. Am J Prev Med. 2012;43(2):201-4.

34. Murtagh S, Rowe DA, Elliott MA, McMinn D, Neslon NM. Predicting active school travel: the role of planned behavior and habit strength. Int J Behav Nutr Phys Act. 2012;9(65).

35. Clemes SA, Biddle SJ. The use of pedometers for monitoring physical activity in children and adolescents: measurement considerations. J Phys Act Health. 2013;10(2):249-262.

36. Carson V, Spence JC. Seasonal variation in physical activity among children and adolescents: a review. Pediatr Exerc Sci. 2010;22:81-92.

37. Mitra R, Faulkner G. There's no such thing as bad weather, just the wrong clothing: climate, weather and active school transportation in Toronto, Canada. Can J Public Health. 2012;103(Suppl. 3):S35-S41. 
38. Adamo K, Prince SA, Tricco AC, Connor-Gorber S, Tremblay MS. A comparison of indirect versus direct measures for assessing physical activity in the pediatric population: a systematic review. Int J Pediatr Obes. 2009;4(1):2-27.

39. Cooper AR, Jago R, Southward EF, Page AS. Active travel and physical activity across the school transition: the PEACH project. Med Sci Sports Exerc. 2012;44:1890-7. 


\section{Legends for Figures and Tables}

Table 1. Descriptive characteristics of the sample at baseline and follow-up.

Table 2. Associations between neighbourhood walkability and participant's primary travel mode at baseline and follow-up.

Table 3. Associations between neighbourhood walkability and step counts at baseline and follow-up 


\section{Table 1.}

\begin{tabular}{|c|c|c|c|}
\hline Variable & Categories & Baseline & Follow-up \\
\hline \multirow[t]{2}{*}{ Gender (n) } & Girls & 24 & 16 \\
\hline & Boys & 24 & 13 \\
\hline \multirow{3}{*}{ Travel mode } & Active & $27(14 \mathrm{G}, 13 \mathrm{~B})$ & $13(8 \mathrm{G}, 5 \mathrm{~B})$ \\
\hline & Inactive & $19(9 \mathrm{G}, 10 \mathrm{~B})$ & $15(7 \mathrm{G}, 8 \mathrm{~B})$ \\
\hline & Missing & $2(1 \mathrm{G}, 1 \mathrm{~B})$ & $1(1 \mathrm{G})$ \\
\hline Distance $(\mathrm{km})$ & $\mathrm{N} / \mathrm{A}$ & $2.3 \pm 2.4$ & $3.9 \pm 3.4$ \\
\hline Walk Score $\uparrow$ around the home & $\mathrm{N} / \mathrm{A}$ & $62.4 \pm 17.8$ & $62.0 \pm 19.3$ \\
\hline Walk Score around the school & $\mathrm{N} / \mathrm{A}$ & $62.0 \pm 20.2$ & $61.5 \pm 27.8$ \\
\hline \multirow{3}{*}{ Average steps/day } & Overall & $16,805 \pm 3,744^{*}$ & $14,071 \pm 3,680 *$ \\
\hline & Girls & $15,235 \pm 2,973$ & $12,728 \pm 3,301$ \\
\hline & Boys & $18,447 \pm 3,820$ & $15,415 \pm 3,662$ \\
\hline
\end{tabular}

Baseline data was collected in May/June 2012 and follow-up data was collected in September/October 2012. G= girls; B = boys. †Walk Score ratings range from 0 to 100 with higher scores indicating greater walkability. Walk Score ratings, distance and steps/day are presented as mean $\pm \mathrm{SD} . *$ denotes statistically significant differences between boys and girls $(\mathrm{p}<0.05)$. Two participants provided insufficient information to allow for the determination of their primary travel mode at baseline and one at follow-up. 
Table 2.

\begin{tabular}{|c|c|c|c|c|c|}
\hline Time point & Walk Score & $\begin{array}{c}\text { Active travelers } \\
(\text { mean SD) }\end{array}$ & $\begin{array}{c}\text { Inactive travelers } \\
(\text { mean SD) }\end{array}$ & OR (95\% CI) & $p$ \\
\hline \multirow{2}{*}{ Baseline } & Home & $61.5 \pm 17.4$ & $62.8 \pm 18.8$ & $0.99(0.96-1.03)$ & .87 \\
\cline { 2 - 6 } & School & $68.3 \pm 18.1$ & $54.7 \pm 19.7$ & $1.04(1.01-1.07)$ & .03 \\
\hline \multirow{2}{*}{ Follow-up } & Home & $76.3 \pm 9.7$ & $51.7 \pm 17.1$ & $1.12(1.03-1.21)$ & .01 \\
\cline { 2 - 6 } & School & $79.5 \pm 1.5$ & $45.9 \pm 31.4$ & $1.29(1.00-1.66)$ & .05 \\
\hline
\end{tabular}

Differences in Walk Score ratings between active and inactive travelers were assessed at baseline (May/June 2012) and follow-up (September/October) using binary logistic regression adjusted for gender. 
Table 3.

\begin{tabular}{|c|c|c|c|c|c|}
\hline Time point & Walk Score & Variable & $\mathrm{F}$ & $p$ & $\eta^{2}$ \\
\hline \multirow{6}{*}{ Baseline } & \multirow{3}{*}{ Home } & Corrected model & 4.28 & .02 & .19 \\
\hline & & Walk Score & 0.04 & .85 & $<.01$ \\
\hline & & Gender & 8.12 & $<.01$ & .18 \\
\hline & \multirow{3}{*}{ School } & Corrected model & 4.91 & .01 & .19 \\
\hline & & Walk Score & 0.71 & .79 & $<.01$ \\
\hline & & Gender & 9.38 & $<.01$ & .18 \\
\hline \multirow{6}{*}{ Follow-up } & \multirow{3}{*}{ Home } & Corrected model & 4.88 & .02 & .30 \\
\hline & & Walk Score & 5.21 & .03 & .19 \\
\hline & & Gender & 6.83 & .02 & .23 \\
\hline & \multirow{3}{*}{ School } & Corrected model & 2.99 & .07 & .21 \\
\hline & & Walk Score & 1.97 & .17 & .08 \\
\hline & & Gender & 5.02 & .04 & .18 \\
\hline
\end{tabular}

Physical activity was measured with SC-StepMX pedometers at baseline (May/June 2012) and follow-up (September/October); that is immediately before and after the school transition. 


\section{Chapter 8. General discussion}

As explained in the introduction, this dissertation had two overarching objectives: 1) to measure

the relationships of AST with PA and health-related outcomes; 2) to examine the correlates of AST immediately before and after the school transition. Therefore, the following discussion consists of two main sections discussing the results of the six studies and situating them within the broader literature. Then, the limitations and strengths of the studies are summarized. Finally, the implications of the studies for future research and interventions are discussed.

\subsection{Relationships of active school transport with physical activity and health-related outcomes:}

Studies 1 to 4 focussed specifically on the association of AST with PA and health-related outcomes, and the key findings are summarized in Table 2. The following sections discuss the associations of AST with: 1) PA levels; 2) body composition indicators; 3) cardiovascular fitness; 4) other measures of physical fitness and CVD risk factors. Then, a fifth section comments on the test-retest reliability of measures of AST, and a sixth one discusses the changes in AST and PA levels across the transition from primary to secondary school. 


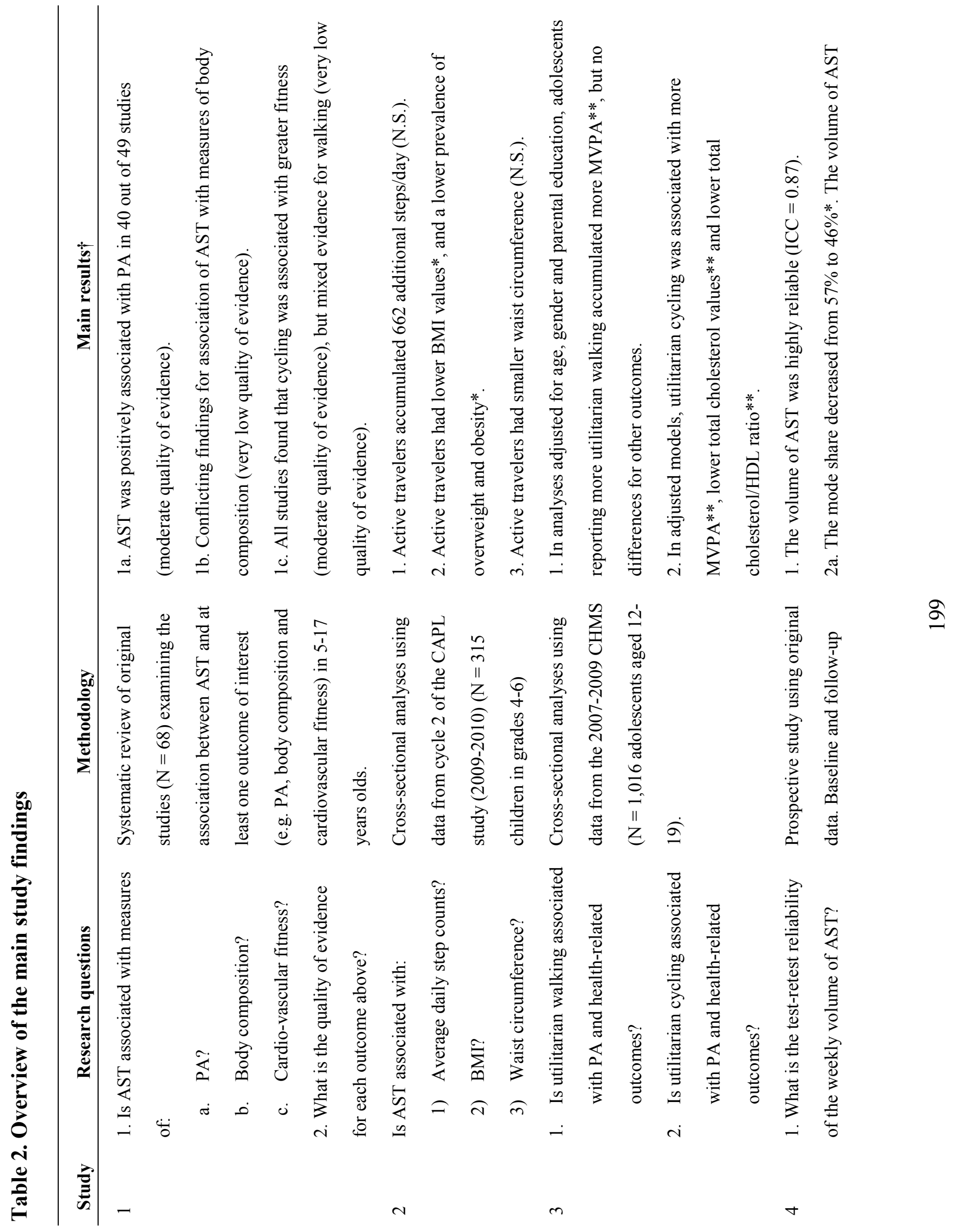



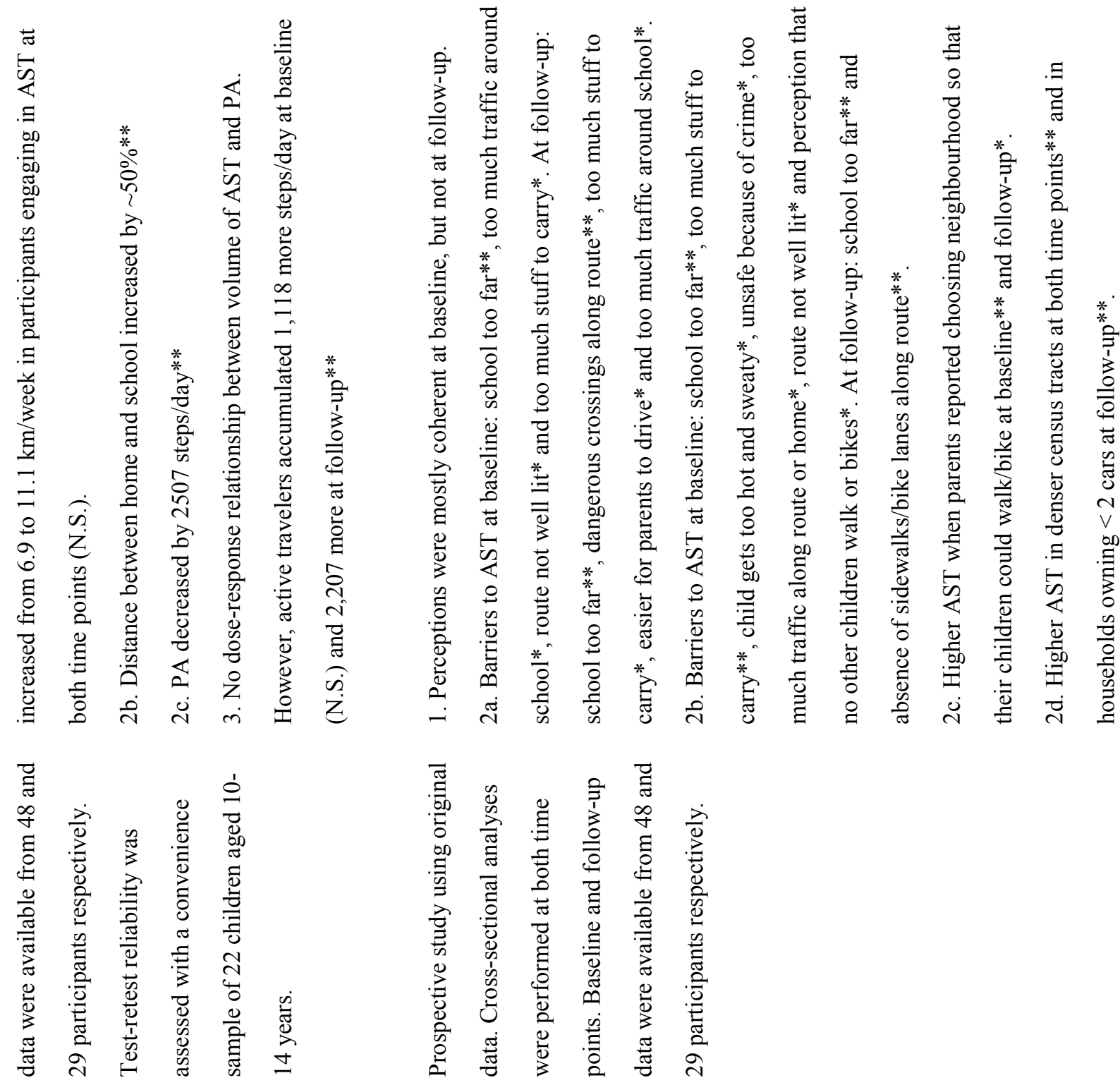

ㄱํㅇ

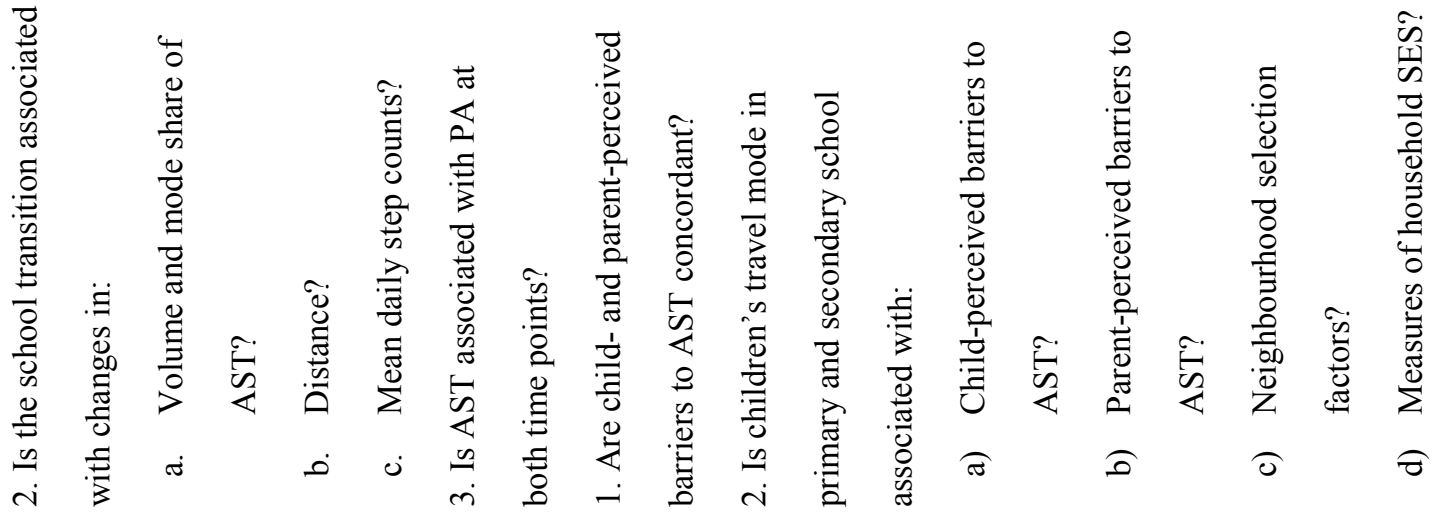




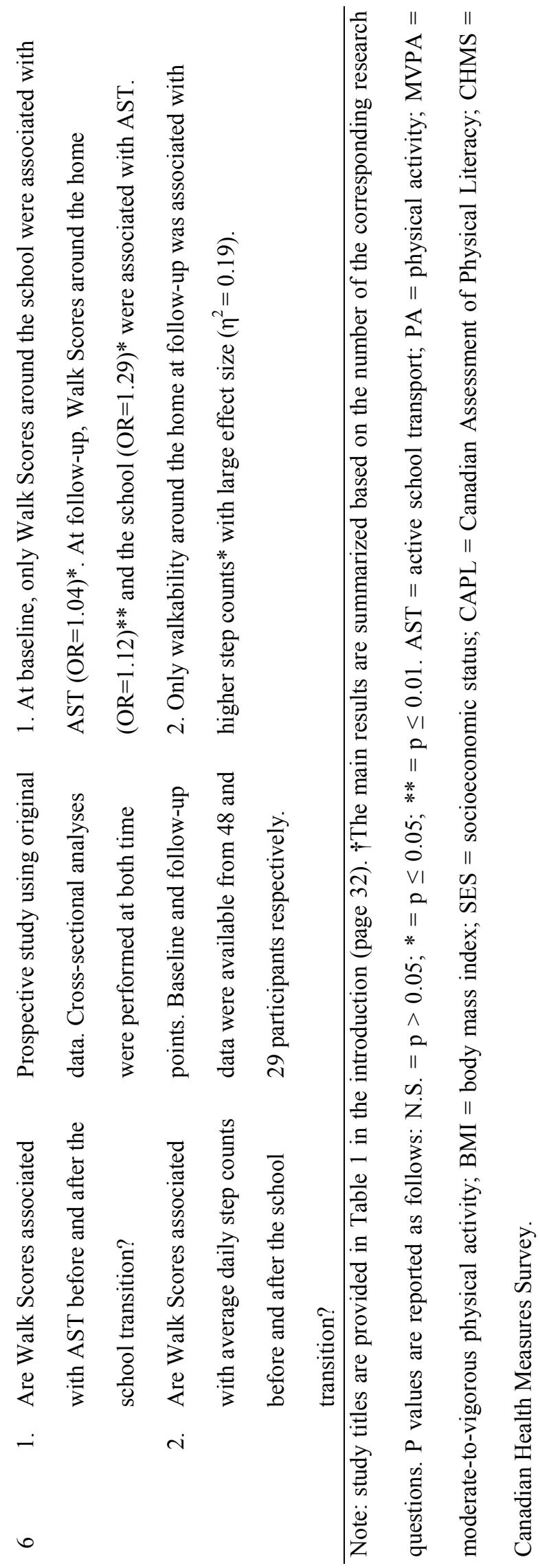


8.1.1 Association between active school transport and physical activity levels. While previous systematic reviews concluded that AST was associated with higher objectively-measured daily PA (Faulkner et al., 2009; Lee et al., 2008), almost all included studies were cross-sectional, and these authors emphasized that future studies should use stronger designs. Four intervention studies met the inclusion criteria of the present systematic review. Of these, 3 studies indicated that walking school bus interventions led to increased PA (Heelan et al., 2009; Mendoza et al., 2011; Sirard et al., 2008). In contrast, McMinn et al. (2012) found no significant differences in daily PA between participants assigned to the experimental group (which received a classroombased intervention consisting of lessons and goal-setting activities led by their teacher) and those assigned to the control group. Of note, participants were 8-9 years old, so they likely had limited independent mobility, and this may have reduced the effectiveness of this educational intervention.

Another important contribution of the present review is the systematic assessment of the quality of evidence using the Effective Public Health Practice Project tool (Effective Public Health Practice Project, 2011) for individual studies and the GRADE approach (Guyatt et al., 2008a, b) for the overall quality of evidence. The present review also included both objective and subjective measures of PA. Of 49 studies that included a measure of PA, 40 have shown that children who engaged in AST were more active than those who were driven to/from school. Twenty eight of these studies have measured PA with accelerometers and the difference in PA between active and inactive travelers ranged from 0 to 45 minutes of MVPA. Notably, 12 out of 14 studies using self-report instruments reported that active travelers were more active.

Given that $81.6 \%$ of included studies (including 2 randomized controlled trials) found that active travelers were more active than passive travelers, that 2 studies had shown evidence 
of a dose-response relationship (van Sluijs et al., 2009; Panter et al., 2011), and that accelerometers and pedometers are known to underestimate physical activity during cycling (Corder et al., 2007), it was concluded that the quality of evidence supporting an association between AST and higher daily PA is moderate. It was deemed premature to conclude that the quality of evidence is high because reported associations were often inconsistent across genders and/or age groups.

Further, two original studies included in this dissertation have examined the association between AST and daily PA levels as measured by pedometers (Studies 2 and 4). In study 2, active travelers accumulated 662 more steps per day than inactive travelers, but this difference was not statistically significant $(p=.23)$ and effect size was small $(\mathrm{d}=0.19)$. It is worth noting that this study included a large proportion of participants recruited in rural areas. In comparison, previous Canadian studies have found that the contribution of AST to daily PA levels is lower in rural areas compared to suburban and urban areas (Pabayo et al., 2012b; Rainham et al., 2012). Of particular interest, Rainham and colleagues (2012) used a combination of global positioning systems and accelerometers to determine the relative contribution of different sources of PA to daily MVPA in adolescents living in rural, suburban and urban areas. They observed that active transportation in general accounted for the greatest proportion of MVPA in adolescents living in urban and suburban areas while the school was the greatest source of MVPA in adolescents from rural areas. It is possible that most children and youth attending rural schools live too far away to engage in AST, irrespective of their inclination toward PA in general.

In study 4, the association between AST and PA levels was assessed immediately before and after the transition from primary to secondary school. Active travelers accumulated 1,118 more steps/day than passive travelers in primary school, but this difference was not significant 
and the effect size was small. This non-significant difference may be due to the low statistical power and to the limitation of pedometers in measuring PA during cycling (Corder et al., 2007). Indeed, cycling was the main travel mode of 10 of the 27 active travelers in primary school. At follow-up, the difference in step counts was almost twice greater than at baseline $(2,207$ steps/day), and the effect size was large. This is a considerable difference as the Canadian Physical Activity Guideline of 60 minutes of daily MVPA corresponds to approximately 12,000 steps/day (Colley et al., 2012). Similarly, two separate cross-sectional studies using comparable methodology suggest that the contribution of the journey to/from school to daily MVPA in British children is much greater in secondary school students compared to primary school students (Cooper et al., 2010; Southward et al., 2012).

Furthermore, study 3 examined the association between active transportation for a broader range of destinations and accelerometry-measured PA in adolescents who participated in the nationally-representative 2007-2009 Canadian Health Measures Survey. Valid accelerometry data was available from 729 participants. Given the large sample size, it was possible to analyse walking and cycling separately (in contrast with studies 2 and 4). In analyses adjusted for age, gender and parental level of education, both walking and cycling were significantly associated with higher daily MVPA. However, differences in sedentary behaviour and light PA between levels of walking and cycling were not significant. These findings are consistent with those of Smith et al. (2012) who observed that children who engaged in active transportation to destinations other than school accumulated more minutes of accelerometry-measured MVPA. Dollman \& Lewis (2007) also noted that children who engaged in AST were $30 \%$ more likely to actively travel to other destinations in their neighbourhood. Thus, AST may be part of a broader habit of walking and cycling. 
In conclusion, the 3 original studies have shown that active travelers accumulate greater amounts of PA than inactive travelers, but in some cases the differences were not statistically significant. The systematic review has also highlighted some inconsistencies in the association between AST and PA, but overall the majority of studies have found that active travelers are significantly more active and that walking school buses interventions can lead to an increase in PA. Hence, these findings support the rationale for promoting AST as a source of physical activity and for implementing interventions such as walking school buses.

\subsubsection{Association between active school transport and body composition indicators. The} systematic review identified 39 studies that assessed the association between AST and different body composition indicators. Of these, 22 found no significant relationship, 14 reported that active travelers had a more favourable body composition and 3 noted that active travelers had a less favourable body composition. Given the inconsistency of study findings, the quality of evidence was rated as very low, suggesting a need for future studies with stronger designs.

Differences in BMI between travel modes were assessed in 38 studies of which 22 found no difference, 13 reported that AST was associated with lower BMI values and 3 reported the opposite. In study 2, active travelers were less likely to be overweight or obese and had significantly lower BMI values than inactive travelers (18.7 vs. $\left.19.9 \mathrm{~kg} / \mathrm{m}^{2}\right)$ with a small effect size $(\mathrm{d}=0.34)$. The difference in BMI remained significant in ANCOVA analyses adjusted for age, gender and step counts. In contrast, study 3 observed that levels of utilitarian walking and cycling were not associated with BMI.

With respect to the relationship between AST and waist circumference, 4 out of 5 papers included in the systematic review found no significant associations with the remaining study 
noting higher waist circumference in active travelers. In study 2 , mean waist circumference was lower by $3.1 \mathrm{~cm}$ among active travelers with a small effect size $(\mathrm{d}=0.29)$, but this difference was not statistically significant. In study 3 , children who engaged in higher amounts of utilitarian walking had significantly higher waist circumference in univariate analyses, but this difference was no longer significant in analyses adjusted for age, gender and parental education. Conversely, adolescents who reported cycling for utilitarian purposes tended to have lower waist circumference, but this difference was not significant.

Study 3 also included measurements of skinfold thickness, but no significant differences were found across levels of walking and cycling. In the systematic review, 4 of the 8 studies that assessed relationships between AST and skinfold thickness found that active travelers were leaner while the 4 other studies found no differences.

Overall, the present research shows mixed evidence regarding associations between AST and body composition indicators. It is possible that for many children, the energy expenditure from AST is insufficient to have a meaningful impact on energy balance. These conflicting findings suggest that further research should monitor AST more precisely than simply asking participants to report their usual travel mode (Larouche \& Saunders, 2012). The continuous measure of the volume of AST developed in study 4 might be useful in this regard. In addition, a few studies have found that cycling, but not walking was associated with more favourable body composition indicators (Bere et al., 2011; Østergaard et al., 2012), suggesting that analyses should be stratified by travel mode when possible.

The lack of control for compensatory behaviours is another important limitation of the literature on the association between AST and body composition indicators. Individuals could compensate for the energy expenditure associated with AST in the rest of the day, either by: 1) 
engaging in less physical activity (PA); 2) engaging in more sedentary behaviour; and/or 3) increasing their energy intake (Larouche \& Saunders, 2012). While the present systematic review (along with studies 3 and 4) provide evidence that active travelers are more active overall, the associations of AST with sedentary behaviour and energy intake remain unclear. Of note, a review of studies employing the doubly-labeled water technique indicated that increases in PA are often offset by compensatory increases in food intake (Westerterp, 2010). PA interventions can also be offset by a decrease in non-exercise activity thermogenesis (e.g. the energy expenditure associated with the maintenance of body posture, fidgeting and movements excluding exercise), thereby limiting their impact on energy expenditure (Tremblay et al., 2007).

8.1.3 Association between active school transport and cardiovascular fitness. The systematic review included 10 studies that examined the association between walking to/from school and cardiovascular fitness. Because Shephard (2008) has suggested that utilitarian walking might not be sufficiently intense to improve cardiovascular fitness in children and youth, results were compiled separately for walking and cycling in the 5 studies that made this analytical distinction. While one study reported that walkers were significantly fitter, the 4 other studies reported no differences in cardiovascular fitness between children who walked to/from school and those who were driven. Therefore, it was concluded that the evidence regarding the association of walking to/from school with cardiovascular fitness in children and youth is of very low quality. Study 3 also found no differences in cardiovascular fitness across levels of utilitarian walking.

In contrast, all 5 studies that examined the association of cycling to/from school with cardiovascular fitness reported that cyclists were significantly fitter. Of particular interest, 
children who started to cycle during the 6-year follow-up of the Danish arm of the European Youth Heart Study had a $9 \%$ greater $\mathrm{VO}_{2}$ max compared to those who did not cycle at any time points. Given the consistency in study findings, the quality of evidence was rated as moderate. Experimental studies are warranted to further increase the quality of evidence. A randomized controlled trial was published after the last update of the systematic review search strategy, but it has suffered from compliance issues with many participants assigned to the control group starting to cycle to school (Børrestad et al., 2012). Irrespective of group allocation, there was a 7.9\% increase in $\mathrm{VO}_{2}$ peak over a 12 -week period. Study 3 has also shown that adolescents who engaged in utilitarian cycling had $\sim 6-10 \%$ higher cardiovascular fitness than those who reported no cycling in unadjusted analyses. However, this association became non-significant in analyses adjusted for age, gender and parental education.

\subsubsection{Associations of utilitarian walking and cycling with other health indicators. Study}

3 was one of the first to assess the relationships of active transportation with indicators of physical fitness (other than cardiovascular fitness) in children and youth. The results indicate no association of levels of walking and/or cycling with performance on the sit and reach and grip strength tests. These findings are somewhat different from those of the Danish youth and sports study (Andersen et al., 2009). They reported that adolescents who cycled to school had better abdominal endurance and trunk flexibility (possibly due to the common use of road bikes by Danish adolescents), but there were no differences in muscle strength. In contrast, there were no associations between walking to school and physical fitness indicators.

Study 3 is, to our knowledge, the first investigation on the relationships of walking and cycling with cardiovascular disease (CVD) risk factors in youth that considered the trip to school 
as well as other destinations (work and errands). No associations were found between levels of walking and CVD risk factors. These findings are consistent with two prospective studies which reported no relationships between walking to/from school and CVD risk factors in participants to the follow-up of the Danish and Swedish arms of the European Youth Heart Study (Andersen et al., 2011; Chillón et al., 2012). However, Pizarro et al. (2013) observed that walking to/from school was associated with a lower likelihood of having a waist circumference greater than the $90^{\text {th }}$ percentile and HDL-cholesterol below $40 \mathrm{mg} / \mathrm{dl}$ in Portuguese children.

With respect to the association between utilitarian cycling and CVD risk factors, Chillón et al. (2012) noted that cycling to school was associated with significantly greater estimated $\mathrm{VO}_{2} \max$, but not with other health outcomes. Andersen and colleagues (2011) observed that children who started to cycle during the 6-year follow-up period had higher estimated $\mathrm{VO}_{2}$ max, a better lipid profile and a lower clustered CVD risk score compared to those who did not cycle at any time point. Moreover, cross-sectional analyses indicated that, at baseline, 15 year old cyclists had higher estimated $\mathrm{VO}_{2}$ max and lower values for triglycerides, total cholesterol/HDL ratio, fasting glucose, insulin resistance and clustered CVD score. In study 3, utilitarian cycling was associated with lower total cholesterol and total cholesterol/HDL ratio.

Together, the findings of study 3 and those of the European Youth Heart Study suggest that in youth, cycling may have a greater effect on CVD risk factors than walking. This may be attributable to the higher PA intensity of cycling compared to walking (Shephard, 2008). Future experimental studies should assess whether AST interventions can lead to a better CVD risk profile. 
8.1.5 Test-retest reliability of different measures of AST. An important limitation identified in the systematic review was the use of inconsistent approaches to categorize children's school travel. For example, some studies classified individuals using AST only once per week as "active travelers" while other used more stringent criteria (i.e. at least $50 \%$ of trips). Thus, a key objective of study 4 was to develop a continuous measure of AST and assess its testretest reliability. The weekly volume of AST was determined as the product of the frequency of AST (e.g. the number of active trips per week) and the distance between home and school, which was estimated based on parent-reported postal codes. It showed high test-retest reliability over 2 consecutive weeks of measurement $(\mathrm{ICC}=0.87)$.

Additionally, a categorical measure of AST was also used to facilitate comparison with other studies. Participant's primary mode of transport (e.g. active vs. inactive) was determined based on the information from the diaries. Participants were classified as active travelers if they used AST for at least $50 \%$ of school trips. This measure also showed high test-retest reliability $($ kappa $=0.77)$, in line with other measures of individuals' habitual travel mode and "hands-up" classroom surveys (Bere \& Bjorkelund, 2009; Evenson et al., 2008; McDonald et al., 2011).

\subsubsection{Changes in active school transport and physical activity across the school}

transition. Study 4 assessed the changes in AST and PA across the transition from primary to secondary school. This major life event has repeatedly been shown to coincide with an important decrease in PA levels (Dumith et al., 2011; Sallis et al., 2000). Yet, only one study has previously examined changes in AST across this transition period (Cooper et al., 2012). They reported that the proportion of children walking to/from school decreased by $16.3 \%$ across the school transition in British youth. Moreover, daily MVPA increased by $16 \%$ in children who 
started to walk to/from school in secondary school compared to those that were driven in primary and secondary school. MVPA decreased by a similar proportion in children who switched from active to inactive travel.

Among children who engaged in AST at both time points, the average volume of AST increased from 6.9 to $11.1 \mathrm{~km} /$ week with a moderate effect size $($ Cohen $\mathrm{d}=0.52)$; however this difference was not statistically significant, likely because of the limited statistical power. The $50 \%$ increase in distance between home and school is likely a key contributor to the observed changes in the volume of AST. In contrast, an $11 \%$ decrease in the mode share of AST was observed across the school transition. These findings suggest that fewer children engaged in AST, but those who did so traveled greater distances.

With respect to daily step counts, an average decrease of 2,507 steps per day was observed across the school transition. Supplementary longitudinal analyses indicated that the decrease in step counts was significantly smaller in children who engaged in AST at follow-up (966 vs. $-4,048$ steps/day; $\mathrm{t}=-3.180 ; p=.004)$. This relationship remained significant in a gender-adjusted ANOVA $(\mathrm{F}=8.36 ; p=.008)$ with no gender by travel mode interaction, and the effect size was large $\left(\eta^{2}=.27\right)$. Additionally, Carver et al. (2011) observed that the strength of the association between the frequency of active transportation (not restricted to the trip to/from school) and MVPA increased over time in an Australian longitudinal study. While the school transition occurred at one point during the follow-up period, the authors did not specifically assess its impact on AST and MVPA. Together, these findings suggest that AST may help attenuate the commonly-observed age-related decline in PA levels, but further studies with longer follow-up periods are warranted to confirm these findings. 
On a related note, Bélanger and colleagues (2009b) examined the longitudinal trends in the practice of 29 different physical activities from grade 7 to 11 . They found that walking was the only activity in which participation did not decrease in both genders. Longitudinal data from the Amsterdam Longitudinal Growth and Health Study (Van Mechelen et al., 2000) show that engagement in lifestyle-embedded PA (including active transportation) is more likely to be maintained into adulthood than sport and leisure-time PA.

Promoting AST during childhood might be especially important given that previous research has shown that habits are a strong predictor of travel modes in children (Murtagh et al., 2012) and adults (Gardner \& Abraham, 2008; Lemieux \& Godin, 2009). Commenting on this issue, Roberts (1995) argued: "With only a handbrake and a handful of birthdays between the passenger's seat and the driver's seat, it may be unrealistic to expect the chauffeured children of today to become the ambulant adults of tomorrow".

\subsection{Correlates of active school transport:}

Despite its potential benefits, many studies have found that the mode share of AST is decreasing (Buliung et al., 2009; Costa et al. 2012; Cui et al., 2011; Grize et al., 2010; McDonald et al., 2011; Trang et al., 2012; van der Ploeg et al., 2008); thus, there is a need for studies to investigate the correlates of AST to inform the development of more effective interventions. In this regard, studies 5 and 6 aimed to address important research gaps with respect to the study of correlates of AST. Of particular interest, no earlier study had examined the correlates of AST immediately before and after the school transition in the same participants. The following sections discuss: 1) the concordance between child- and parent-perceived barriers to AST; and 2) 
the correlates of AST, using the five levels of influence of the ecological model presented in the introduction.

\subsubsection{The concordance between child- and parent-perceived barriers to active school}

transport. Although recent theoretical models posit that children and youth may have some input into the travel mode decision making (Panter et al., 2008; Pont et al., 2011), few studies have simultaneously assessed child- and parent-perceived barriers to AST. In their literature review, Panter and colleagues (2008) identified only 2 studies that examined perceived barriers in both children and parents (Timperio et al., 2004, 2006), but the concordance was not reported. In their validation study, Forman et al. (2008) compared parents' and adolescents' perception of barriers grouped into three subscales (environmental, planning/psychosocial, and safety), and found high agreement for each subscale. A recent study noted that the perception of 5 different barriers was similar between children and parents, except in less walkable areas where parents expressed greater concerns (Napier et al., 2011).

In the present study, children's and parents' perceptions of 19 different barriers were compared at baseline and follow-up. While significant correlations were observed for 14 items at baseline, there was much lower agreement at follow-up with only 4 significant correlations. Using the Forman (2008) subscales ${ }^{5}$, a similar pattern was observed using data from study 5. At baseline, children's and parent's perceptions were significantly correlated for the environmental, planning/psychosocial, and safety subscales (rho $=.65, .53$ and .49 respectively; all $p<.01$ ), but at follow-up, a significant correlation was found only for the environmental subscale (rho $=.59$; $p<.01)$. These findings could be explained by a novelty effect because children and parents were probably less familiar with the route to/from school and the school surroundings at follow-

\footnotetext{
${ }^{5}$ Internal consistency values for these subscales are provided in Appendix 10.
} 
up. Alternatively, it is possible that as children are granted more independent mobility, their parents may become less aware of their whereabouts, including the characteristics of the route to/from school.

8.2.2 Personal characteristics. Study 4 was one of the first studies to examine the effect of the school transition on rates of AST, and a significant decrease in the mode share of AST was observed. These findings are consistent with cross-sectional reports which consistently indicate lower mode shares of AST in secondary school students in North America (Buliung et al., 2009; Evenson et al., 2003; Fulton et al., 2005; Pabayo et al., 2008). Similarly, data from the Canadian National Longitudinal Survey of Children and Youth shows an increase in AST up to 10 years of age followed by a marked decrease in adolescence (Pabayo et al., 2011). In contrast, Cooper and colleagues (2006) reported that $66 \%$ of 15 year old youth cycled to school compared to $38 \%$ of 9 year old children in Odense (Denmark) while walking rates were similar between age groups ( 20-25\%). A longitudinal survey of Belgian children and youth has also shown that rates of cycling increase with age (Cardon et al., 2012). Together, these studies suggest that the influence of age (or school level) on AST vary by country.

In study 3 , the proportion of 16-19 year old adolescents who reported walking at least 5 hours per week for utilitarian purposes was about twice as high as that of younger adolescents (29.2\% vs. $14.7 \%)$. This suggests that utilitarian walking to/from destinations other than school may account for a larger proportion of older adolescents' active transportation. In particular, active transportation to work is likely more common among the 16-19 year old age group. However, a slightly higher proportion of 16-19 year olds reported that they did not engage in any utilitarian cycling ( $83.7 \%$ vs. $77.7 \%$ ) during the last three months. 
In study 5 , there were no gender differences in the mode share of AST, neither at baseline nor at follow-up. This finding differs from most North American studies which have reported that boys were more likely to engage in AST (Evenson et al., 2003; Fulton et al., 2005; Gropp et al., 2012; McMillan et al., 2006), but this should be interpreted with caution given the small sample size. Study 3 also demonstrated no gender differences in levels of utilitarian walking. However, almost 4 times more boys reported at least an hour of utilitarian cycling per week. These results are consistent with those of studies indicating that boys cycle 2-3 times more than girls (McDonald, 2012; Roberts et al, 1997). Similar gender gaps have been found in adults in countries where the mode share of cycling is very low such as Australia, Canada, the UK and the US (Pucher \& Buehler, 2008; Garrard et al., 2012). Interestingly, women cycle as much as men in The Netherlands, Denmark and Germany where cycling is much safer and cycling infrastructure more developed (Pucher \& Buehler, 2008). Girls also cycled as much as boys in Odense (Denmark) and Umeå (Sweden) (Cooper et al., 2006; Roberts et al., 1997). Previous research suggests that women are typically more risk-averse than men (Garrard et al., 2012), and that parents are more restrictive with respect to girls' independent mobility (Valentine, 1997).

Perceived convenience may also have a strong influence on travel mode decisions (Faulkner et al., 2010; Lorenc et al., 2008). In study 5, a greater proportion of inactive travelers reported that AST involves too much planning ahead, but this proportion did not significantly differ from that of the active travelers. However, at follow-up, motorized travelers were more likely to report that it's easier for their parents to drive them on the way to something else ( $46.7 \%$ vs. $7.7 \%)$ compared to active travelers. There was a similar trend at baseline ( $44.4 \%$ vs. 19.2\%), but the difference was not significant. 
8.2.3 Social environment. With respect to parent-perceived convenience, higher percentages of parents reported that "it is easier to drive" and that walking or biking to school "involves too much planning ahead" at both time points. However, these perceptions were not significantly associated with their child's travel mode, probably because of the limited statistical power. Previous literature has shown that parent-perceived convenience is an important correlate of travel mode choices (Faulkner et al., 2010; McDonald \& Aalborg, 2009; McMillan, 2007; Panter et al., 2010a). For instance, Faulkner et al. (2010) concluded that once parents have decided whether their child needs to be escorted to/from school, they choose the travel mode that they consider the quickest and most convenient.

Previous research has also indicated that perceived social norms were associated with children's travel mode (Lee et al., 2013; McMillan et al., 2007). For example, McMillan (2007) noted that children were $48 \%$ more likely to engage in AST when their parents perceived that their family would approve their decision to allow their child to walk to school. In study 5 , few children and parents agreed with the two relevant questionnaire items ("no other children walk or bike to school" and "it is not considered cool to walk or bike"). Nevertheless, the percentage of parents reporting that no other children walk or bike to school was significantly higher in children using motorized travel mode at follow-up (20.0\% vs. 0\%).

In contrast with previous studies (Evenson et al., 2003; Gropp et al., 2012; McDonald, 2008; Pabayo et al., 2008, 2011), study 5 indicated few associations between household SES and children's travel mode. This may be the result of the small sample size combined with limited variability in SES in this sample (e.g. most parents had university education and were working full time). However, children were more likely to engage in AST when their parents owned less than 2 cars at follow-up. This observation is consistent with earlier research indicating a negative 
relationship between car ownership and the likelihood of AST (DiGuiseppi et al., 1998; Ewing et al., 2004; Gropp et al., 2012).

In study 5 , less than $10 \%$ of parents indicated that the presence of stray dogs or bullying/teasing were barriers to their child's engagement in AST. In addition, few children and parents reported that AST is unsafe because of crime (e.g. strangers, gangs, drug dealers). Nevertheless, parent's crime-related concerns were significantly associated with AST, but only at baseline. In their literature review, Panter et al. (2008) found conflicting evidence regarding the association between personal safety and AST. This could be due to many factors including the high prevalence of parental personal safety concerns (Timperio et al., 2004), and to the higher mode share of AST in low SES neighbourhoods which tend to have higher crime rates (Pabayo et al., 2012a; Rossen et al., 2011).

Study 5 was also one of the first to examine the role of parental neighbourhood selection factors on children's school travel. Two previous cross-sectional studies have noted that children were more likely to engage in AST if their parents reported that they chose to live in their current neighbourhood so that their children could walk to school (Lee et al., 2013; Mammen et al., 2012). An important contribution of the present study is that this association was examined before and after the school transition and it was significant in both cases. In addition, children were more likely to engage in AST at follow-up if their parents chose their neighbourhood because "there are shops and restaurants you can walk or cycle to". These results are consistent with studies of residential self-selection in adults (Frank et al., 2007; Handy et al., 2006), suggesting that future studies should control for parental neighbourhood selection where appropriate. 
8.2.4 Public policies and institutional practices. At both time points, children were less likely to engage in AST when they reported that they have too much stuff to carry. This association was also found for parent reports at baseline, but not at follow-up. While few studies have examined this issue, the present findings are consistent with a survey of Texas parents (Lee et al., 2013) and with focus groups involving primary school children and parents in North Carolina (Ahlport et al., 2008). The quantity of material that children must carry to/from school likely depends to a certain extent on school practices. For example, using USB sticks and tablets instead of heavy textbooks could help minimizing backpack loads. Alternatively, the use of bike panniers could eliminate the need for children to carry heavy items on their back, and "cyclingfriendly" schools could facilitate this by providing space for safely storing bikes and panniers.

Six percent of parents who participated in a large US survey indicated that school policies prohibited AST (Martin \& Carlson, 2005). Similarly, some school principals whose school was involved in the Canadian Health Behavior in School-aged Children survey reported that bicycles were not allowed on school property (O'Loghlen et al., 2011). While study 5 did not specifically assess school policies, a greater provision of bike racks was observed in the schools located in denser census tracts than in the other schools (Richard Larouche, unpublished observations). Moreover, a greater percentage of inactive travelers agreed with the statement that there is nowhere to leave a bike safely around school, but this association was not statistically significant.

Higher level policies may also influence rates of AST. For example, US researchers indicated that in states requiring crossing guards around schools, walking school buses were more likely to be implemented and principals reported higher rates of AST (Chriqui et al., 2012; Turner et al., in press). Furthermore, AST was more prevalent in states requiring lower speed 
limits around schools (Chriqui et al., 2012). In contrast, school siting policies recommending that schools be built on large lots (e.g. Council of Educational Facility Planners International, 1991) may have a negative influence on the mode share of AST (Environmental Protection Agency, 2003). Indeed, large schools are typically constructed in the outskirts of cities, where land is cheaper, leading to larger distances between home and school (Ewing et al., 2004). As a result, McDonald (2008) estimated that only about 20\% of US students lived within $1.6 \mathrm{~km}$ from their school. This situation represents a major challenge to interventions that aim to increase the mode share of AST such as Safe Routes to School and walking school buses.

8.2.5 Built environment. Literature reviews have concluded that there is consistent evidence showing that the mode share of AST decreases while distance between home and school increases (Panter et al., 2008; Stewart, 2011; Wong et al., 2011). For example, distance explained about $47 \%$ of the decrease in the mode share of AST in the US between 1969 and 2001 (McDonald, 2007b). Given the very strong and consistent associations between objectively-measured distance and AST, recent studies have examined the correlates of AST among children and youth living within a distance assumed to be walkable (Gropp et al., 2012; Lee et al., 2013; Mammen et al., 2012). Of particular interest, Lee and colleagues (2013) used a case-control study design to assess the correlates of AST among children living within 2 miles $(3.2 \mathrm{~km})$ away from school. Children who walked or traveled by car were matched based on distance, and they lived fewer than 200 feets $(61$ metres $)$ from each other $(p=0.99)$. Still, parents whose child walked to school were significantly more likely to perceive that the school was close enough for walking. This suggests that the perception of distance is associated with travel behaviours over and above objectively-measured distance. 
In study 5, children's and parents' perception that the school is too far and objectivelymeasured distance were both strong barriers to AST before and after the school transition. Moreover, the average objectively-measured distance increased by about $50 \%$ across the school transition, likely contributing to the observed decrease in the mode share of AST. The increase in distance across the school transition is consistent with the observation that secondary schools generally have larger catchment areas (Environmental Protection Agency, 2003).

Previous systematic reviews examining the associations of other built environment constructs with AST and PA have generally reported conflicting findings (Ding et al., 2011; Wong et al., 2011). Many hypotheses have been proposed to explain these results. First, important methodological differences in the assessment of built environment characteristics (Wong et al., 2011) have been noted, including inconsistencies in geocoding, different buffer ${ }^{6}$ size and shape, and discrepancies in the quality of road and infrastructure data. There may also be discrepancies between perceived and objective measures of the built environment (Ding et al., 2011). For example, Lee et al. (2013) found that parents whose child engaged in active vs. inactive travel had different perceptions of their neighbourhood despite living in the same environment. Second, in their review of the literature, Ding et al. (2011) reported that measures of PA varied widely across studies. Estimates of PA levels differ markedly between self-reports and objective measures (Adamo et al., 2009). Third, Panter et al. (2008) argued that failure to account for potential moderators such as age, gender, and SES may partly explain the inconsistent evidence. Fourth, the influence of the built environement may vary depending on the context of physical activities, so an environment that facilitates leisure-time PA may be less conductive to AST (Giles-Corti et al., 2009). For example, although low density neighbourhoods

\footnotetext{
${ }^{6}$ The buffer is the area within which built environment constructs are measured [see Wong et al. (2011) for review].
} 
with large lots and cul-de-sacs may facilitate active outdoor play, they may offer little destinations accessible by walking or cycling.

In study 5, children who attended one of the two primary schools located in census tracts with higher population density were much more likely to engage in AST than those attending schools located in less dense census tracts, and this difference persisted at follow-up. In denser areas, it might be easier to provide neighbourhood schools in which a high proportion of students live within a walkable distance. For instance, McDonald (2008) reported that the direct influence of density on AST was very small. However, density was strongly associated with distance which, in turn, was the strongest correlate of AST. In study 5, the two primary schools located in denser census tracts also had higher Walk Score ratings (75 and 85) than those located in less dense census tracts (ratings of 35 and 60), and similar differences were found at follow-up.

Study 5 also suggests that perceiving heavy traffic as a barrier to AST was generally associated with motorized travel to/from school, although the location of traffic-related concerns (e.g. along the route or around the home or around the school) tended to differ between children and parents. Many other studies have shown that road safety concerns were associated with reduced odds of AST (Carver et al., 2008; Kerr et al., 2006; Lee et al., 2013; Panter et al., 2010a; Voorhees et al., 2010), although null associations have also been reported (Carson et al., 2010; Timperio et al., 2004).

Before and after the school transition, a greater proportion of inactive travelers and their parents reported that there are no sidewalks or bike lanes along the route; however, differences were statistically significant only for parent reports at follow-up. Several studies have found that perceived availability of sidewalks and/or objectively-measured sidewalk coverage was positively associated with AST (Ewing et al., 2004; Fulton et al., 2005; Gropp et al., 2012; Kerr 
et al., 2006), although others reported no differences (Evenson et al., 2006; Mota et al., 2007). Of particular interest, an investigation of Safe Routes to School projects in California indicated that built environment changes such as adding or improving sidewalks, traffic lights, pedestrian crossings and bike paths can lead to an increase in AST (Boarnet et al., 2005). However, this study was limited by its retrospective design.

At baseline, reporting insufficient lighting along the route was associated with engaging in motorized travel, but this association was not significant at follow-up. In addition, the percentage of respondents who reported dangerous crossings along the route was consistently higher among inactive travelers; however this difference was significant only for child reports at follow-up. Notably, an Australian prospective study found that youth whose parents perceived that there were sufficient pedestrian crossings in their neighbourhood were 2.5 times more likely to increase their frequency of AST over a 2-year follow-up period (Hume et al., 2009).

Systematic reviews have observed that few studies have used composite measures of neighbourhood walkability (Ding et al., 2011; Wong et al., 2011). This is an important research gap because studies of adults have repeatedly found significant associations between measures of neighbourhood walkability and active transportation (Gauvin et al., 2005; Saelens \& Handy, 2008; Sallis et al., 2006, 2009). Furthermore, Sallis and colleagues (2009) have suggested that the influence of built environment characteristics on adults' PA may be additive, suggesting that while individual characteristics may explain only a very small proportion of the variance in AST and PA, their combined explanatory power may be larger.

In study 6, measures of Walk Scores around participant's home (using parent-reported postal codes as a proxy for residential address) and the school that they attended were computed. Then, the associations of Walk Scores with AST and PA before and after the school transition 
were assessed. At baseline, walkability around the school was associated with an increased likelihood of AST, but it was not associated with PA. Walkability around the home was associated with neither AST nor PA. However, at follow-up, higher Walk Score ratings around the home and the school were both related to an increased likelihood of AST, and the odds ratios were larger. Moreover, walkability around the home was associated with higher step counts, with a large effect size. These preliminary findings are supportive of Giles-Corti and colleagues' (2009) hypothesis that neighbourhood walkability may have a stronger influence in adolescents than in children.

Similarly, a US cross-sectional study reported that built environment characteristics were more strongly associated with walking in 12-15 years old youth compared to younger participants (Frank et al., 2007). Additional analyses indicated that the influence of built environment constructs on walking was weaker among non-white youth, those living in lowincome families and those whose parents did not own a car (Kerr et al., 2007). The authors argued that these individuals may have limited travel options; thus rendering characteristics of the built environment less relevant in terms of travel mode choices. In contrast, a US survey found no association between a measure of neighbourhood walkability (including residential density, intersection density, land use mix, and floor area ratio) and adolescents' PA levels and BMI (Norman et al., 2006). In a survey of Belgian youth, a similar measure was associated with PA only in low-SES neighbourhoods, and it was not associated with AST (De Meester et al., 2012).

It is worth noting that these walkability measures are heavily influenced by correlates of adults' travel mode choices such as density, diversity and design (see Cervero \& Kockelman, 1997 for theoretical model), whose influence on children's travel patterns may be more complex. 
For example, an Australian study found a strong interaction between traffic density and street connectivity (Giles-Corti et al., 2011). Specifically, in neighbourhoods with high connectivity and low traffic, children were 3.63 times more likely to engage in AST than in areas with low connectivity and high traffic. In contrast, high connectivity did not facilitate AST in neighbourhoods with heavy traffic.

From a methodological point of view, the Walk Score algorithm considers a large range of amenities located within a $1.6 \mathrm{~km}$ linear buffer. However, amenities within a 400 meter buffer receive maximal points, and amenities located further away receive fewer points following a distance decay function (Aleisha Jacobson, personal communication). Interestingly, Mitra \& Buliung (2012) recently reported that the influence of objectively-measured built environment constructs varies with the shape and the size of the buffer. In their study, the influence of the built environement was larger when using a 400 meter buffer compared to larger buffers. They also argued that when using larger buffers, the variance in built environment constructs may be reduced, thereby decreasing the likelihood of observing significant associations. Thus, the use of a distance decay function might be judicious.

8.2.6 Physical environment. Study 5 included two perceived physical environment variables. First, children's perception that they get too hot and sweaty was not associated with their travel pattern at any time points. However, children were more likely to use motorized travel modes when their parents perceived that they get too hot and sweaty. It is unclear whether parents were more likely to offer their child a ride on hot days. Second, no relationships were found between the perception that there are too many hills along the way and children's travel mode at any time points. Given the relatively flat topography of the city of Ottawa, this result is 
not surprising. Previous research has suggested that the presence of hills is a significant barrier only in specific cases such as for young children (Timperio et al., 2006) or for cycling (Ahlport et al., 2008).

It is worth noting that the occurrence of seasonal variations in AST remains unclear. While Børrestad et al. (2011) reported a large decrease in cycling during the winter in Norway, the majority of cyclists switched to walking. In the Greater Toronto Area, walking rates remained stable in the winter while cycling was deemed too uncommon to be included in analyses (Mitra \& Faulkner, 2012). Spontaneous and unplanned activities may be more likely to vary with season and weather conditions than scheduled activities such as organized sports (Bélanger et al., 2009a) and/or AST (Mitra \& Faulkner, 2012).

\subsection{Limitations and strengths of the studies:}

8.3.1. Limitations. Several limitations should be considered when interpreting the present findings. In the systematic review, it was not possible to conduct a meta-analysis because included studies had used inconsistent measures of AST. In addition, studies in which PA was measured with accelerometers have used many different cut-points. In this regard, Cain et al. (2013) reported that cut-points used to determine MVPA with the Actigraph accelerometer in the pediatric population ranged from 400 to 3600 counts per minute. The use of cut-points corresponding to an energy expenditure greater than 4 metabolic equivalents (METs) may also lead to the categorization of most walking as light intensity PA; for example, the Compendium of Energy Expenditures for Youth (Ridley et al., 2008) assigns a 3.6 METs value for walking at a moderate pace. Moreover, despite known differences in PA intensity between walking and cycling (Shephard, 2008), many included studies have combined walkers and cyclists in their 
analyses due to the low mode share of cycling and/or the small sample size. This limitation also pertains to studies 2 and 4 .

The cross-sectional design is an important limitation of studies 2 and 3 because it is not possible to determine the direction of the observed relationships. Hence, one may argue that fitter and more active children may be more inclined to engage in AST. However, randomized controlled trials have shown that the implementation of walking school buses led to increases in AST and PA (Mendoza et al., 2011; Sirard et al., 2008), and prospective studies have shown that switching from inactive to active travel can increase PA (Cooper et al., 2012; Saksvig et al., 2012; Smith et al., 2012) and cardiovascular fitness (Andersen et al., 2011; Chillón et al., 2012).

In studies 3 and 4, the difference in PA between active and inactive travelers may have been underestimated to some extent because pedometers and accelerometers are known to underestimate PA during cycling (Corder et al., 2007). Given the very low mode share of cycling in study 2 , it is very unlikely that this limitation had an influence on the results.

In study 4, the observed decline in PA levels across the school transition may be partly explained by seasonal variations in PA. Previous research shows that children and youth's PA levels tend to vary markedly between seasons (Carson \& Spence, 2010; Goodman et al., 2012), but a prospective study found that increases in PA during warmer months were insufficient to compensate for decreases during the winter, so that PA decreased by $7 \%$ per year in adolescence (Bélanger et al., 2009a). To minimize seasonal bias, follow-up data collection was conducted as early as possible in the school year (September and October).

The small sample size of the Active Transportation Transition Study (e.g. studies 4-6) has several implications. First, statistical power was low, thereby increasing the likelihood of type II error. Second, the sample may not be representative of the population and generalizability may 
be limited given that all participating schools were in Ottawa and the parents were likely more educated compared to the broader population. Third, the small sample size limited adjustment for potential confounders (i.e. school clustering, measures of SES and car ownership). Fourth, despite the longitudinal study design, it was not feasible to assess the predictors of changes in travel mode across the school transition (only 3 participants switched from inactive to active travel modes, and 6 did the opposite). Similarly, in the broader literature, very few studies have assessed correlates of travel behaviour change (Hume et al., 2009). Thus, replication of this study design in larger samples is warranted to confirm the present findings.

On a different note, internal consistency was low for child-perceived barriers at baseline (Cronbach $\alpha$ values from .67 to .71) based on Forman and colleagues' (2008) subscales indicating that children reports in primary should be interpreted with caution.

Finally, while Walk Score ratings provide a composite measure of neighbourhood walkability, it does not provide information on which objectively-measured built environment constructs are associated with AST and PA. Provided that sufficient funding is available, future studies should include both individual and composite measures of the built environment.

8.3.2. Strengths. The present studies also have important strengths. First, study 1 is, to date, the most comprehensive systematic review on the association of AST with PA and healthrelated outcomes in children and youth. Specifically, it is the first systematic review on this topic that included: 1) intervention studies; 2) all measurements of PA; and 3) a systematic assessment of the quality of evidence.

In addition, studies 2, 3 and 4 have used objective measures of PA and body composition indicators. This minimized social desirability and recall biases that are common when using self- 
reported measures. Specifically, such biases can lead to an overestimation of PA levels and height, and an underestimation of body weight (Adamo et al., 2009; Connor Gorber et al., 2007).

To date, study 3 is the largest study that assessed the association between active transportation and CVD risk factors in children and youth. Moreover, the three earlier studies on this topic have only considered the trip to/from school (Andersen et al., 2011; Chillón et al., 2012; Pizarro et al., 2013). In addition, data collection occurred throughout the year, thereby minimizing the risk that population estimates of PA are biased by seasonal effects.

The Active Transportation Transition Study (e.g. studies 4, 5 and 6) was one of the first investigations of the influence of the school transition on rates of AST. The development of a reliable measure of the volume of AST addressed a key limitation highlighted in the systematic review. The results provide preliminary evidence suggesting that AST may contribute to attenuate the well-described age-related decline in PA levels. Other noteworthy contributions include the assessment of a wide variety of potential correlates of AST before and after the school transition including: 1) child- and parent-perceived barriers to AST; 2) household SES; 3) parental neighbourhood selection factors; and 4) neighbourhood walkability. Of particular interest, the influence of neighbourhood walkability on AST and PA levels was stronger after the school transition, suggesting that the influence of the built environment might be stronger in adolescents than in children. Another important contribution is the observation that children were more likely to engage in AST at both time points when their parents indicated that they had chosen to live in their neighbourhood so that their children could easily walk and cycle to school. This suggests that parental neighbourhood choices may have a long-lasting influence on their children's school travel. 


\subsection{Directions for future research:}

Because the vast majority of the research on active transportation in children and youth has focused solely on the trip to/from school, little is known about active transportation to nonschool destinations and how it relates to PA and health-related outcomes. Study 3 indicated some differences in levels of utilitarian walking and cycling according to gender and age groups. Specifically, older adolescents (16-19 year old) tended to report higher amounts of utilitarian walking than their younger counterparts (12-15 year old). This finding is in contrast with the literature showing that rates of AST are lower in high school students (Evenson et al., 2003; Fulton et al., 2005; Gropp et al., 2012; McMillan et al., 2006). This suggests that active transportation to non-school destinations may increase during adolescence, and this may provide an important source of PA in a period characterized by a steep decline in leisure-time PA (Dumith et al., 2011). Moreover, the correlates of active transportation may vary according to trip purposes, providing further rationale for considering children and youth's travel to destinations other than school.

The results of study 4 suggested that AST may contribute to attenuate the age-related decline in PA levels. This underscores a need for future prospective studies with longer followup periods. Additionally such studies could investigate changes in AST and PA levels associated with other important life events where large decreases in PA have been reported such as the beginning of post-secondary studies and entry into the labour market (Allender et al., 2008; Engberg et al., 2012; Larouche et al., 2012).

With respect to the promotion of AST, a recent systematic review identified 14 intervention studies and their effectiveness has been heterogeneous, although most of them led to modest increases in AST (Chillón et al., 2011). Most interventions were limited by the absence 
of control groups; thereby precluding causal inferences. Also, most interventions were implemented in primary schools, so there is a clear need for future research on the development and evaluation of interventions aiming to increase AST in secondary school students. For example, adult-led walking school buses might be inappropriate for adolescents, but walking or cycling "buddy" systems could be a feasible way to foster social support for - and engagement in - AST in youth as well as in adults (Borghese et al., 2011).

While several studies have investigated the correlates of children and youth's current travel mode, research on travel mode behaviour change is particularly scarce (Hume et al., 2009), underscoring a need for future longitudinal studies. Of particular interest, the school transition may be characterized by a change in travel habits and increasing independent mobility. This could present a good opportunity to investigate the predictors of behaviour change to inform the development of more effective interventions.

Related to this, further research is needed to explore mediators and moderators of travel mode choices. McMillan's (2005) theoretical framework posits that variables such as traffic and neighbourhood safety and car availability can mediate the relationship between the built environment and AST. However, formal mediation analyses have seldom been performed (Stewart et al., 2011). Theoretical frameworks also identify variables such as age, gender, SES, distance and social norms as potential moderators (McMillan, 2005; Panter et al., 2008). In this regard, Panter et al. (2010a) found that distance moderated the associations of road safety and parental attitudes with children's travel mode. Specifically, attitudes were generally more important for shorter trips whereas safety concerns were more influent for longer trips. This makes intuitive sense because longer trips entail greater exposure to traffic (Roberts et al., 1997). 
Of particular interest, future studies should investigate whether independent mobility moderates or mediates the relationship between the theory of planned behaviour constructs and children's travel patterns. Murtagh and colleagues (2012) found that intention and perceived behavioural control explained $10 \%$ of the variance in the number of steps accumulated during the trip to school in 8-9 years old children, and habit strength explained an additional $6 \%$. However, at this age, children likely had limited independent mobility, and the proportion of explained variance might have been stronger in more autonomous children.

The conceptual model presented in the introduction assumes that travel mode decisions are taken based on the characteristics of the environment (i.e. individual characteristics, the social environment, institutional policies and practices, built environment, and physical environment). However, there is a clear need for future studies to better understand the school travel decision-making process (Faulkner et al., 2010), especially the extent to which children's input varies with age. A combination of qualitative and quantitative methodologies would be useful for this purpose. First, in depth interviews with parents and children could help to gain new insight into family interactions and the key elements underlying travel mode choices. Further qualitative work could help inform the development of more refined survey instruments to assess the decision-making process and other correlates of AST in larger samples. Then, intervention studies could be designed to address some of the key elements identified in this process.

Additionally, the conceptual model suggests that engagement in active transportation has a positive impact on measures of environmental quality (i.e. greenhouse gases and vehicle exhaust gases emissions), to the extent that it replaces motorized travel (de Nazelle et al., 2011; Larouche, 2012; Litman, 2013; Marshall et al., 2010; Woodcock et al., 2009). Conversely, 
participation in other forms of PA may have negative environmental impacts if it generates additional motorized trips (Litman, 2013). Therefore, environmental co-benefits may represent a plus-value for active transportation compared to other activities. In this regard, de Nazelle and colleagues (2011) emphasized that future studies should quantify the environmental co-benefits of active transportation interventions more accurately.

The conceptual model also underscores that measures of environmental quality can influence health outcomes. For example, a scientific statement from the American Heart Association concluded that airborne particulate matter is associated with an increased likelihood of cardiovascular and respiratory disease (Brook et al., 2010). In addition, the short-term transport strategy that was put in place during the 1996 Atlanta Summer Olympic Games led to reduced pollution levels and to a decrease in hospital admissions for acute asthma events in children (Friedman et al., 2001). Future studies should assess the extent to which pollution reductions achieved through mode shifts from motorized to active travel may influence other health outcomes.

\subsection{Implications for interventions and policies:}

Given the increasing body of evidence showing that active transportation is associated with greater PA levels and other health-related outcomes, governments and organizations should promote utilitarian walking and cycling as an important, inexpensive, accessible and environmentally-friendly source of PA (Active Healthy Kids Canada, 2013; British Medical Association, 2012). Because children and youth must attend school 5 days per week, using active travel modes for at least part of the trip can be an easy way to integrate habitual PA into their lifestyle, and involvement in activities such as walking and cycling may be more likely to be 
sustained throughout adolescence and into adulthood (Bélanger et al., 2009b; Van Mechelen et al., 2000).

The Canadian Health Measures Survey analyses indicated that almost four times as many boys than girls engage in utilitarian cycling for at least an hour per week. These results suggest that interventions should be developed to promote cycling among girls. Given that no gender difference exists in countries were cycling infrastructure is safer and more expensive (Cooper et al., 2006; Garrard et al., 2012; Pucher \& Buehler, 2008; Roberts et al., 1997), the development of safer cycling infrastructure may help alleviate the gender gap in Canada. While this may be viewed as expensive, research has shown that investments in cycling infrastructure are generally cost-effective (Cavill et al., 2008; Gotschi, 2011).

Long distance between home and school has repeatedly been found to be a strong barrier to AST and it may deprive children from an opportunity to develop their independent mobility and to engage in PA. To address this situation, the recent Canadian child- and youth-friendly land-use and transport planning guidelines (Gilbert \& O’Brien, 2010) recommend that urban planners and school administrators consider children's travel to a greater extent. With respect to school trips, these guidelines recommend that "school policies and practices favour walking and cycling, and other modes of active transport for trips to and from school, and also regular public transport where this is available and appropriate". This entails that efforts should be made to reduce children's car travel as much as possible "because of poor in-vehicle air quality and opportunities lost to exercise, gain independence, and experience neighbourhoods". In this regard, novel strategies could be developed for children who live beyond what is considered a walkable distance to accomplish at least a part of their school using AST. For example, as part of a Safe Routes to School intervention in Atlanta (Georgia), a church located $0.5 \mathrm{~km}$ away from 
the school offered space for parents to park their car and walk with their children (Henderson et al., 2013). An advantage of developing such partnerships is that one does not have to wait for infrastructure projects to be completed.

Three interventions included in the systematic review have found that walking school bus interventions led to increases in PA levels. Additionally, qualitative studies from New Zealand suggest that perceived benefits of walking school buses include social interactions with other children, traffic reduction, time savings for the parents, improved health, and the development of children's road safety awareness (Kearns et al., 2003; Kingham and Ussher, 2007). However, the long term viability of such interventions may be compromised by the reliance on volunteers (Kingham and Ussher, 2005). Interestingly, Mammen and colleagues (2012) reported that $67 \%$ of parents who drive their children to/from school in the Greater Toronto Area indicated that they would be more likely to allow them to engage in AST if walking school buses were organized by the school. Implementation of walking school buses should be encouraged by governmental policies and facilitated by schools, and strategies to reward or recognize walking school bus "conductors" should be developed.

Safe Routes to School programs can also be implemented to address the road safety barriers noted in study 5 and in the broader literature (Carver et al., 2008; Kerr et al., 2006; Lee et al., 2013; Panter et al., 2010a; Voorhees et al., 2010). These programs emerged in Denmark in the late 1970s, when the country had the highest rate of child pedestrian injuries in Europe (Appleyard, 2003). To address this issue, the city of Odense invested in the creation of an extensive network of pedestrian and bicycle paths, narrowed roads, added traffic islands, and mandated all of its 45 schools to identify and address specific road-safety issues. After ten years, child pedestrian and cyclist casualties had decreased by $80 \%$ and this program was implemented 
at the national level. School travel planning was also very successful in the UK where pedestrian and cyclist casualties fell by $77 \%$ and $28 \%$ respectively (Appleyard, 2003).

In the US, $\$ 612$ million have been invested in Safe Routes to School initiatives between 2005 and 2009 (Watson and Dannenberg, 2008) and similar initiatives, albeit at a smaller scale, have been implemented in Canada (Bergeron et al., 2011; Green Communities Canada, 2010, 2012). An evaluation of the impact of Safe Routes to School interventions has recently been conducted in New York City (DiMaggio \& Li, 2013). The authors noted a 44\% decrease in pedestrian injuries among school-aged children in "experimental" census tracts while no changes were observed in control census tracts. However, this study did not assess changes in travel modes. While not necessarily implemented with the objective of promoting AST, the introduction of $32 \mathrm{~km} / \mathrm{h}$ speed limits in London (UK) has led to a $50 \%$ decrease in road casualties among children (Grundy et al., 2009). Such decreases in the risk of accidents could help alleviate parental road safety concerns.

Analyses from the Canadian arm of the Health Behavior in School-aged Children survey indicated that $42 \%$ of schools were located on roads with speed limits greater than $60 \mathrm{~km} / \mathrm{h}$, and this proportion was as high as $91 \%$ for rural schools (O'Loghlen et al., 2011). Almost $40 \%$ of rural schools were located on a street without a sidewalk. Furthermore, less than $40 \%$ of surveyed schools reported having a designated car-free zone, organizing walk to school days and identifying safe routes to school in any way. These proportions are much lower than those noted in a British study (Panter et al., 2010b).

At the international level, the United Nations General Assembly adopted a resolution that proclaimed a Decade of Action for Road Safety (2011-2020). This was accompanied by the publication of the 2013 Global Status Report on Road Safety (WHO, 2013) which indicates that 
road accidents represents the $8^{\text {th }}$ leading cause of death worldwide, and it may become the $5^{\text {th }}$ cause by 2030 unless urgent action is taken. Emphasis is placed among other things on the adoption and enforcement of lower speed limits (especially in urban areas), and more stringent policies pertaining to drinking and driving. The report presents detailed data for most countries around the world and it may be used as a key tool to advocate for road safety legislations.

Finally, additional efforts may be needed to raise awareness about existing programs and interventions aiming to promote AST. Indeed, previous research in the Greater Toronto Area has indicated that parental awareness of AST programs in elementary school is very low, but awareness is positively associated with children's engagement in AST (Arbour-Nicitopoulos et al., 2011). Social marketing campaigns could be useful in this regard. Similarly, given that active transportation may become an increasingly important source of PA in adolescents, social marketing campaigns could also be used to promote walking and cycling. 


\section{Conclusion}

In conclusion, the vast majority of studies examining the association between AST and daily PA have shown that active travelers were more active, including 3 intervention studies of walking school buses. In addition, active trips to/from other destinations (i.e. work and errands) may also be an increasingly important contributor to adolescents PA levels. The Active Transportation Transition Study suggests that engagement in AST may help to attenuate the agerelated decline in PA levels, although future studies with larger samples and longer follow-up periods are warranted to confirm this finding. Therefore, AST should be promoted as an important source of PA in children and youth.

Engagement in AST may also be associated to other health-related outcomes in children and youth. For example, the systematic review shows that cycling to/from school is consistently associated with higher cardiovascular fitness. A similar trend was observed in the secondary analyses from the Canadian Health Measures Survey, but this association was no longer significant in models adjusted for confounders and multiple comparisons. However, these analyses have shown that utilitarian cycling was associated with a more favourable lipid profile, as reflected by lower values for total cholesterol and total cholesterol/HDL cholesterol ratio.

Nevertheless, only $25-35 \%$ of Canadian children and youth regularly engage in AST, and several studies have reported that the mode share of AST has decreased markedly during the last few decades. These trends suggest that many barriers need to be addressed. In this regard, there is consistent evidence showing that distance between home and school (either perceived or objectively-measured) is the strongest correlate of AST. Changes to school siting policies and urban planning practices will likely be needed to reduce distance, but in the meantime, strategies 
can be developed to encourage children and youth to do at least a portion of their school trip using AST.

Still, a sizable proportion of children who live within a walkable distance do not engage in AST. Despite the small sample size, the present research identified several associations between children- and parent-perceived road safety concerns and a higher proportion of motorized trips. Programs such as walking school buses and Safe Routes to School, as well as the implementation of traffic calming measures may help to address these concerns. In addition, the perception of having too much stuff to carry was associated with motorized travel, suggesting that strategies to reduce backpack load and/or use more efficient means to carry the loads (e.g. bike panniers) may be useful. The present dissertation also suggests that future studies should continue to monitor car ownership as a correlate of AST, and further consider the role of parental neighbourhood selection factors in predicting children's travel mode.

Finally, the association of neighbourhood walkability with AST and PA was stronger in secondary schools compared to primary schools. To date, no other longitudinal study has examined whether the associations of walkability with AST and PA changes over time; thus future prospective studies are warranted. There is also a clear need for additional research on active transportation to/from non-school destinations which might become an increasingly important source of PA during adolescence. 


\section{References}

Abbott, R.A., MacDonald, D., Nambiar, S., \& Davies, P.S.W. (2009). The association between walking to school, daily step counts and meeting step targets in 5- to 17-years-old Australian children. Pediatric Exercise Science, 21, 520-532.

Active Healthy Kids Canada (2013). Are we driving our kids to unhealthy habits? 2013 Active Healthy Kids Canada Report Card on Physical Activity in Children and Youth. Toronto, ON: Active Healthy Kids Canada.

Adamo, K., Prince, S.A., Tricco, A.C., Connor-Gorber, S., \& Tremblay, M.S. (2009). A comparison of indirect versus direct measures for assessing physical activity in the pediatric population: a systematic review. International Journal of Pediatric Obesity, 4 (1), 2-27.

Ahlport, K.N., Linnan, L., Vaughn, A., Evenson, K.R., \& Ward, D.S. (2008). Barriers and facilitators of walking and bicycling to school: formative results from the Non-motorized Travel Study. Health Education \& Behavior, 35 (2), 221-244.

Allender, S., Huchinson, L., \& Foster, C. (2008). Life-change events and participation in physical activity: a systematic review. Health Promotion International, 23 (2), 160-172.

Andersen, L.B., Harro, M., Sardinha, L.B., Froberg, K., Ekelund, U., Brage, S., \& Anderssen, S.A. (2006). Physical activity and clustered cardiovascular risk in children: a crosssectional study (The European Youth Heart Study). Lancet, 368, 299-304.

Andersen, L.B., Lawlor D.A, Cooper, A.R., Froberg, K., \& Anderssen, S.A. (2009). Physical fitness in relation to transport to school in adolescents: the Danish youth and sports study. Scandinavian Journal of Medicine and Science in Sports, 19, 406-411. 
Andersen, L.B., Wedderkopp, N., Kristensen, P., Moller, N.C., Froberg, K., \& Cooper, A.R. (2011). Cycling to school and cardiovascular risk factors: a longitudinal study. Journal of Physical Activity and Health, 8, 1025-1033.

Anderssen, S.A., Cooper, A.R., Riddoch, C., Sardinha, L.B., Harro, M., Brage, S., \& Andersen, L.B. (2007). Low cardiorespiratory fitness is a strong predictor for clustering of cardiovascular disease risk factors in children independent of country, age and sex. European Journal of Cardiovascular Prevention \& Rehabilitation, 14, 526-531.

Appleyard, B. (2003). Planning Safe Routes to School. Planning, 69 (5), 34-37.

Arbour-Nicitopoulos, K., Faulkner, G.E.J., Buliung, R., Lay, J., \& Stone, M. (2011). Parental awareness of elementary school travel programs. Preventive Medicine, 52, 281-282.

Ajzen, I. (1991). The theory of planned behavior. Organizational behavior and human decision processes, 50, 179-211.

Basterfield, L., Adamson, A.J., Parkinson, K.N., Maute, U., Li, P.X., \& Reilly, J.J. (2008). Surveillance of physical activity in the UK is flawed: validation of the Health Survey for England Physical Activity Questionnaire. Archives of disease in childhood, 93 (12), $1054-1058$.

Basterfield, L., Adamson, A.J., Pearce, M.S., Reilly, J.J. (2011). Stability of habitual physical activity and sedentary behavior monitoring by accelerometry in 6- to 8-year-olds. Journal of Physical Activity and Health, 8 (4), 543-547.

Bauman, A., Allman-Farinelli, M., Huxley, R., \& James, W.P.T. (2008). Leisure-time physical activity alone may not be a sufficient public health approach to prevent obesity - A focus on China. Obesity Reviews, 9, 119-126. 
Bauman, A., Finegood, D.T., \& Matsudo, V. (2009). International perspectives on the physical inactivity crisis - structural solutions over evidence generation? Preventive Medicine, 49, 309-312.

Bélanger, M., Gray-Donald, K., O'Loughlin, J., Paradis, G., \& Hanley, J. (2009a). Influence of weather conditions and season on physical activity in adolescents. Annals of Epidemiology, 19, 180-186.

Bélanger, M., Gray-Donald, K., O’Loughlin, J., Paradis, G., \& Hanley, J. (2009b). Participation in organized sports does not slow declines in physical activity during adolescence. International Journal of Behavioral Nutrition and Physical Activity, 6 (22).

Bere, E., \& Andersen, L.B. (2009). Why no support for an association between active commuting to school and weight status in the literature. Journal of Physical Activity and Health, 6, 533-534.

Bere, E., \& Bjorkelund, L.A. (2009). Test-retest reliability of a new self reported comprehensive questionnaire measuring frequencies of different modes of adolescents commuting to school and their parents commuting to work - the ATN questionnaire. International Journal of Behavioral Nutrition and Physical Activity, 6 (68).

Bere, E., Oenema, A., Prins, R.G., Seiler, S., \& Brug, J. (2011). Longitudinal associations between cycling to school and weight status. International Journal of Pediatric Obesity, 6, 182-187.

Bergeron, J., Paquette, M., Rodrigue, S., \& Avard, M. (2011). Second rapport annuel d'évaluation des activités réalisées dans le cadre du programme « Mon école à pieds, à vélo ». Montréal, QC: Université de Montréal. 
Biddle, S.J.H., \& Avare, M. (2011). Physical activity and mental health in children and adolescents: a review of reviews. British Journal of Sports Medicine, 45, 886-895.

Boarnet, M., Anderson, C.L., Day, K., McMillan, T., \& Alfonzo, M. (2005). Evaluation of the California Safe Routes to School legislation: urban form changes and children's active transportation to school. American Journal of Preventive Medicine, 28 (Suppl. 2), 134140.

Borghese, M., Brooks, M., Earl, S., \& Naddaf, J. (2011). Ride forward - an evaluation of cycling in two Ottawa neighborhoods. Interdisciplinary Journal of Health Sciences, 2 (2), 7-13.

Børrestad, L.A.B., Andersen, L.B., \& Bere, E. (2011). Seasonal and socio-demographic determinants of school commuting. Preventive Medicine, 52 (2), 133-135.

Børrestad, L.A.B., Østergaard, L., Andersen, L.B., \& Bere, E. (2012). Experiences from a randomized controlled trial on cycling to school: does cycling increase cardiorespiratory fitness. Scandinavian Journal of Public Health, 40 (3), 245-252.

British Medical Association (2012). Healthy transport = healthy lives. London: British Medical Association. Retrieved May 2, 2013, from http://bma.org.uk/transport

Brook, R.D., Rajagopalan, S., Pope III, A., Brook, J.R., Bhatnagar, A. et al. (2010). Particulate matter air pollution and cardiovascular disease: an update to the scientific statement from the American Heart Association. Circulation, 121, 2331-2378.

Bronfenbrenner, U. (1979). The ecology of human development: experiments by nature and design. Cambridge, MA: Harvard University Press.

Buliung, R.N., Mitra, R., \& Faulkner, G. (2009). Active school transportation in the Greater Toronto Area, Canada: An exploration of trends in space and time (1986-2006). Preventive Medicine, 48, 507-512. 
Buliung, R., Faulkner, G., Beesley, T., \& Kennedy, J. (2011). School travel planning: mobilizing school and community resources to encourage active school transportation. Journal of School Health, 81, 704-712.

Cain, K.L., Sallis, J.F., Conway, T.L., Van Dyck, D., \& Calhoon, L. (2013). Using accelerometers in youth physical activity studies: a review of methods. Journal of Physical Activity and Health, 10, 437-450.

Cardon, G.M., Maes, L.R.D., Haerens, L.L., \& de Bourdeaudhuij, I.M.M. (2012). Bicycling to school during the transition from childhood to adolescence: a six-year longitudinal study. Pediatric Exercise Science, 24 (3), 369-383.

Carr, L.J., Dunsiger, S.I., \& Marcus, B.H. (2010). Walk Score ${ }^{\mathrm{TM}}$ as a global estimate of neighborhood walkability. American Journal of Preventive Medicine, 39 (5), 460-463.

Carson, V., Kuhle, S., Spence, J.C., \& Veugelers, P.J. (2010). Parent's perception of neighborhood environment as a determinant of screen time, physical activity and active transport. Canadian Journal of Public Health, 101 (2), 124-127.

Carson, V., \& Spence, J.C. (2010) Seasonal variation in physical activity among children and adolescents: a review. Pediatric Exercise Science, 22, 81-92.

Carver, A., Timperio, A., \& Crawford, D. (2008). Playing it safe: the influence of neighbourhood safety on children's physical activity (a review). Health \& Place, 14 (2), 217-227.

Carver, A., Timperio, A.F., Hesketh, K.D., Ridgers, N.D., Salmon, J.L., \& Crawford, D.A. (2011). How is active transport associated with children's and adolescents' physical activity over time? International Journal of Behavioral Nutrition and Physical Activity, $8,126$. 
Cavill, N., Kahlmeier, S., Rutter, H., Racioppi, F., \& Oja, P. (2008). Economic analyses of transport infrastructure and policies including health effects related to cycling and walking: systematic review. Transport Policy, 15, 291-304.

Cervero, R., \& Kockelman, K. (1997). Travel demands and the 3Ds: density, diversity and design. Transportation Research Part D: Transport and Environment, 2 (3), 199-219.

Chapman, L. (2007). Transport and climate change: A review. Journal of Transport Geography, 15 (5), 354-367.

Chillón, P., Evenson, K.R., Vaughn, A., \& Ward, D.S. (2011). A systematic review of interventions for promoting active transportation to school. International Journal of Behavioral Nutrition and Physical Activity, 8 (10).

Chillón, P., Ortega, F.B., Ruiz, J.R., Evenson, K.R., Labayen, I., et al. (2012). Bicycling to school is associated with improvements in physical fitness over a 6-year follow-up period in Swedish children. Preventive Medicine, 55 (2), 108-112.

Chriqui, J.F., Taber, D.R., Slater, S.J., Turner, L., Lowrey, K.M., \& Chaloupka, F.J. (2012). The impact of state safe routes to school-related laws on active travel to school policies and practices in U.S. elementary schools. Health \& Place, 18 (1), 8-15.

Colley, R.C., Garriguet, D., Janssen, I., Craig, C.L., Clarke, J., \& Tremblay, M.S. (2011). Physical activity of Canadian children and youth: Accelerometer results from the 2007 to 2009 Canadian Health Measures Survey. Health Reports, 22 (1), 15-24.

Colley, R.C., Janssen, I., \& Tremblay, M.S. (2012). Daily step target to measure adherence to physical activity guidelines in children. Medicine \& Science in Sports \& Exercise, 44 (5), 977-982. 
Connor Gorber, S., Tremblay, M., Moher, D., \& Gorber, B. (2007). A comparison of direct vs. self-report measures for assessing height, weight, and body mass index: a systematic review. Obesity Reviews, 8, 307-326.

Cooper, A.R., Page, A.S., Foster, L.J., \& Qahwaji, D. (2003). Commuting to school: are children who walk more active. American Journal of Preventive Medicine, 25 (4), 273-276.

Cooper, A.R., Anderson, L.B., Wedderkopp, N., Page, A.S., \& Froberg, K. (2005). Physical activity levels of children who walk, cycle, or are driven to school. American Journal of Preventive Medicine, 29 (3), 179-184.

Cooper A.R., Wedderkopp, N., Wang, N., Andersen, L.B., Froberg, K., \& Page, A.S. (2006). Active travel to school and cardiovascular fitness in Danish children and adolescents. Medicine \& Science in Sports \& Exercise, 38 (10), 1724-1731.

Cooper, A.R., Wedderkopp, N., Jago, R., Kristensen, P.L., Moller, N.C., et al. (2008). Longitudinal associations of cycling to school with adolescent fitness. Preventive Medicine, 47 (3), 324-328.

Cooper, A.R., Page, A.S., Wheeler, B.W., Griew, P., Davis, L., Hillsdon, M., \& Jago, R. (2010). Mapping the walk to school using accelerometry combined with a global positioning system. American Journal of Preventive Medicine, 38 (2), 178-183.

Cooper, A.R., Jago, R., Southward, E.F., \& Page, A.S. (2012). Active travel and physical activity across the school transition: the PEACH project. Medicine \& Science in Sports \& Exercise, 44, 1890-1897.

Corder, K., Brage, S., \& Ekelund, U. (2007). Accelerometers and pedometers: methodology and clinical applications. Current Opinion in Clinical Nutrition \& Metabolic Care, 10 (5), $597-603$. 
Costa, F.F., Silva, K.S., Schmoelz, C.P., Campos, V.C., \& de Assis, M.A.A. (2012).

Longitudinal and cross-sectional changes in active commuting to school among Brazilian schoolchildren. Preventive Medicine, 55 (3), 212-214.

Council of Educational Facility Planners International. (1991). Guide for planning educational facilities. Scottsdale, AZ: Council of Educational Facility Planners International.

Craig, C.L., Shields, M., LeBlanc, A.G., \& Tremblay, M.S. (2012). Trends in aerobic fitness among Canadians, 1981 to 2007-2009. Applied, Physiology, Nutrition and Metabolism, $37(3), 511-519$.

Cui, Z., Bauman, A., \& Dibley, M.J. (2011). Temporal trends and correlates of passive commuting to and from school in children from 9 provinces in China. Preventive Medicine, 52 (6), 423-427.

Dellinger, A., \& Staunton, C.E. (2002). Barriers to children walking and biking to school United States, 1999. Morbidity and Mortality Weekly Reports, 51, 701-704.

De Meester, F., Van Dyck, D., De Bourdeaudhuij, I., Deforche, B., Sallis, J.F., \& Cardon, G. (2012). Active living neighborhoods: is neighborhood walkability a key element for Belgian adolescents? BMC Public Health, 12, 7.

de Nazelle, A., Nieuwenhuijsen, M.J., Anto, J.M., Brauer, M., Briggs, D. et al. (2011). Improving health through policies that promote active travel: A review of evidence to support health impact assessment. Environment International, 37 (4), 766-777.

DiGuiseppi, C., Roberts, I., Li, L., \& Allen, D. (1998). Determinants of car travel on daily journeys to school: cross sectional survey of primary school children. British Medical Journal, 316, 1426-1428. 
DiMaggio, C., \& Li, G. (2013). Effectiveness of a Safe Routes to School program in preventing school-aged pedestrian injury. Pediatrics, 131, 290-296.

Ding, D.G., Sallis, J.F., Kerr, J., Lee, S., \& Rosenberg, D.E. (2011). Neighborhood environment and physical activity among youth: a review. American Journal of Preventive Medicine, $41(4), 442-55$.

Dollman, J., Lewis, N.R. (2007). Active transport to school as part of a broader habit of walking and cycling among South Australian youth. Pediatric Exercise Science, 19 (4), 436-443.

Dumith, S.C., Gigante, D.P., Domingues, M.R., \& Kohl III, H.W. (2011). Physical activity change during adolescence: a systematic review and a pooled analysis. International Journal of Epidemiology, 40, 685-698.

Effective Public Health Practice Project. (2011). Quality Assessment Tool for Quantitative Studies. Retrieved July 12, 2011, from http://www.ephpp.ca/Tools.html

Ekelund, U., Luan, J., Sherar, L.B., Esliger, D.W., Griew, P., \& Cooper, A.R. (2012). Moderate to vigorous physical activity and sedentary time and cardiometabolic risk factors in children and adolescents. Journal of the American Medical Association, 307, 704-712.

Engberg, E., Alen, M., Kukkonen-Harjula, K., Peltonen, J.E., Tikkanen, H.O., \& Pekkarinen, H. (2012). Life events and changes in leisure-time physical activity: a systematic review. Sports Medicine, 42, 433-437.

Environmental Protection Agency. (2003). Travel and environmental implications of school siting. Washington, DC: Environmental Protection Agency.

Evenson, K.R., Huston, S.L., McMillen, B.J., Bors, P., \& Ward, D.S. (2003). Statewide prevalence and correlates of walking and bicycling to school. Archives of Pediatric and Adolescent Medicine, 157 (9), 887-892. 
Evenson, K.R., Birnbaum, A.S., Bedimo-Rung, A.L., Sallis, J.F., Voorhees, C.C., Ring, K., \& Elder, J.P. (2006). Girls' perception of physical environmental factors and transportation: reliability and association with physical activity and active transport to school. International Journal of Behavioral Nutrition and Physical Activity, 3, 28.

Evenson, K.R., Neelon, B., Ball, S.C., Vaughn, A., \& Ward, D.S. (2008). Validity and reliability of a school travel survey. Journal of Physical Activity \& Health, 5 (Suppl. 1), S1-S15.

Ewing, R., Schroeer, W., \& Greene, W. (2004). School location and student travel: analysis of factors affecting mode choice. Transportation Research Record, 1895, 55-63.

Faulkner, G.E.J., Buliung R.N., Flora, P.K., \& Fusco, C. (2009). Active school transport, physical activity levels and body weight of children and youth: a systematic review. Preventive Medicine, 49, 3-8.

Faulkner, G.E.J., Richichi, V., Buliung, R.N., Fusco, C., \& Moola, F. (2010). What's “quickest and easiest?": parental decision making about school trip mode. International Journal of Behavioral Nutrition and Physical Activity, 7, 62.

Frank, L., Kerr, J., Chapman, J., \& Sallis, J. (2007). Urban form relationships with walk trip frequency and distance among youth. American Journal of Health Promotion, 21 (Suppl. 4), 305-11.

Frank, L.D., Saelens, B.E., Powell, K.E., \& Chapman, J.E. (2007). Stepping towards causation: do built environments or neighborhood and travel preferences explain physical activity, driving, and obesity? Social Science and Medicine, 65, 1898-1914.

Frank, L.D., Greenwald, M.J., Winkelman, S., Chapman, J., \& Kavage S. (2010). Carbonless footprints: promoting health and climate stabilization through active transportation. Preventive Medicine, 50 (1), 99-105. 
Friedman, M.S., Powell, K.E., Hutwagner, L., Graham, L.M., \& Teague, W.G. (2001). Impact of changes in transportation and commuting behaviors during the 1996 Summer Olympic Games in Atlanta on air quality and childhood asthma. Journal of the American Medical Association, 285 (7), 897-905.

Fulton, J.E., Shisler, J.L., Yore, M.M., \& Caspersen, C.J. (2005). Active transportation to school: Findings from a national survey. Research Quarterly for Exercise \& Sport, 76, 352-357.

Fyhri, A., Hjorthol, R., Mackett, R.L., Fotel, T.N., \& Kytta, M. (2011). Children's active travel and independent mobility in four countries: development, social contributing trends and measures. Transport Policy, 18, 703-710.

Gardner, B., \& Abraham, C. (2008). Psychological correlates of car use: a meta-analysis. Transportation Research Part F, 11, 300-311.

Garrard, J., Handy, S. \& Dill, J. (2012). Women and cycling. In J. Pucher \& R. Buehler (Eds.), City Cycling (pp. 211-234). Cambridge, MA: Massachusetts Institute of Technology Press.

Gauvin, L., Richard, L., Craig, C.L., Spivock, M., Riva, M., et al. (2005). From walkability to active living potential: an "ecometric" validation study. American Journal of Preventive Medicine, 28 (Suppl. 2), 126-133.

Gilbert, R., \& O'Brien, C. (2010). Canadian child- and youth-friendly land-use and transport planning guidelines. Winnipeg, MB: Centre for Sustainable Transportation. Retrieved May 16, 2013, from http://www.kidsonthemove.ca/uploads/Canadian\%20Guidelines,\%202.pdf 
Giles-Corti, B., Kelty, S.F., Zubrick, S.R., \& Villanueva, K.P. (2009). Encouraging walking for transport and physical activity in children and adolescents: how important is the built environment? Sports Medicine, 39 (12), 995-1009.

Giles-Corti, B., Wood, G., Pikora, T., Learnihan, V., Bulsara, M., et al. (2011). School site and the potential to walk to school: the impact of street connectivity and traffic exposure in school neighborhoods. Health \& Place, 17, S45-S50.

Goodman, A., Paskins, J., \& Mackett, R. (2012). Day length and weather effects on children's physical activity and participation in play, sports, and active travel. Journal of Physical Activity and Health, 9, 1105-1116.

Gotschi, T. (2011). Costs and benefits of bicycling investments in Portland, Oregon. Journal of Physical Activity and Health, 8 (Suppl. 1), S49-S58.

Green Communities Canada. (2010). Canadian school travel planning pilot test final report. Green Communities Canada: Peterborough, ON. Retrieved May 21, 2013, from http://www.saferoutestoschool.ca/sites/default/files/STP\%20Pilot\%20Test $\% 20$ National\% 20Summary\%20Report.pdf

Green Communities Canada. (2012). Children's mobility, health and happiness: a Canadian school travel planning model: 2012 National Results - Executive Summary. Retrieved May 21, 2013, from http://www.saferoutestoschool.ca/downloads/Executive\%20SummaryCLASP\%20Results-May\%202012.pdf

Grize, L., Bringolf-Isler, B., Martin, E., \& Braun-Fahrländer, C. (2010). Trends in active transport to school among Swiss school children and its associated factors: three cross- 
sectional surveys 1994, 2000 and 2005. International Journal of Behavioral Nutrition and Physical Activity, 7, 28.

Gropp, K., Pickett, W., \& Janssen, I. (2012). Multi-level examination of correlates of active transportation to school among youth living within 1 mile of their school. International Journal of Behavioral Nutrition and Physical Activity, 9, 124.

Guyatt, G.H., Oxman, A.D., Vist, G.E., Kunz, R., Falck-Ytter, Y., Alonso-Coello, P., \& Schünemann, H.J. (2008). GRADE: An emerging consensus on rating quality of evidence and strength of recommendations. British Medical Journal, 336, 924-926.

Guyatt, G.H., Oxman, A.D., Vist, G.E., Kunz, R., Falck-Ytter, Y., \& Schünemann, H.J. (2008). GRADE: What is "quality of evidence" and why is it important to clinicians? British Medical Journal, 336, 995-998.

Haines, A., McMichael, A.J., Smith, K.R., Roberts, I., Woodcock, J. et al. (2010). Public health benefits of strategies to reduce greenhouse-gas emissions: Overview and implications for policy makers. Lancet, 374 (9707), 2104-2114.

Hallal, P., Andersen, L.B., Bull, F.C., Guthold, R., Haskell, W., et al. (2012). Physical activity levels of the world population: surveillance progress, gaps and prospects. Lancet, 380 (9838), 247-257.

Handy, S., Cao, X., \& Mokhtarian, P. (2006). Self-selection in the relationship between the built environment and walking: Empirical evidence from Northern California. Journal of the American Planning Association, 72 (1), 55-74.

Heelan, K.A., Abbey, B.M., Donnelly, J.E., Mayo, M.S., \& Welk, G.J. (2009). Evaluation of a walking school bus for promoting physical activity in youth. Journal of Physical Activity and Health, 6, 560-567. 
Henderson, S., Tanner, R., Klanderman, N., Mattera, A., Webb, L.M., \& Steward, J. (2013). Safe Routes to School: a public health practice success story - Atlanta, 2008-2010. Journal of Physical Activity \& Health, 10, 141-142.

Hillman, C., Erickson, K.I., \& Kramer, A.F. (2008). Be smart, exercise your heart: exercise effects on brain and cognition. Nature Reviews Neuroscience, 9, 58-65.

Hillman, M., Adams, J., \& Whitelegg, J. (1990). One false move: A study of children's independent mobility. London: PSI Publishing.

Hinckson, E.A., \& Badland, H.M. (2011). School travel plans: preliminary evidence for changing school-related patterns in elementary school children. American Journal of Health Promotion, 25 (6), 368-371.

Hohepa, M., Scragg, R., Schofield, G., Kolt, G.S., \& Schaaf, D. (2007). Social support for youth physical activity: importance of siblings, friends and school support across a segmented school day. International Journal of Behavioral Nutrition and Physical Activity, 4, 54.

Hume, C., Timperio, A., Salmon, J., Carver, A., Giles-Corti, B., \& Crawford, D. (2009). Walking and cycling to school: Predictors of increase among children and adolescents. American Journal of Preventive Medicine, 36 (3), 195-200.

Jacobs, J. (1961). The death and life of great American cities. New York, NY: Random House. Jago, R., Wedderkopp, N., Kristensen, P.L., Moller, N.C., Andersen, L.B., Cooper, A.R., \& Froberg, K. (2008). Six-year change in youth physical activity and effect on fasting insulin and HOMA-IR. American Journal of Preventive Medicine, 35 (6), 554-560.

Janssen, I., Boyce, W.F., Simpson, K., \& Pickett, W. (2006). Influence of individual- and arealevel measures of socioeconomic status on obesity, unhealthy eating, and physical inactivity in Canadian adolescents. American Journal of Clinical Nutrition, 83, 139-145. 
Janssen, I., Shields, M., Craig, C.L., \& Tremblay, M.S. (2011). Prevalence and secular trends in abdominal obesity in Canadian adolescents and adults, 1981 to 2007-2009. Obesity Reviews, 12 (6), 397-405.

Jiménez-Pavón, D., Kelly, J., \& Reilly, J.J. (2010). Associations between objectively measured habitual physical activity and adiposity in children and adolescents: Systematic review. International Journal of Pediatric Obesity, 5, 3-18.

Kearns, R.A., Collins, D.C.A., \& Neuwelt, P.M. (2003). The Walking School Bus: Extending children's geographies? Area, 35 (3), 285-292.

Kerr, J., Rosenberg, D., Sallis, J.F., Saelens, B.E., Frank, L.D., \& Conway, T.L. (2006). Active commuting to school: associations with environment and parental concerns. Medicine \& Science in Sports \& Exercise, 38 (4), 787-794.

Kerr, J., Frank, L., Sallis, J.F., \& Chapman, J. (2007). Urban form correlates of pedestrian travel in youth: differences by gender, race-ethnicity and household attributes. Transportation Research Part D: Transport and environment, 12 (3), 177-182.

Kingham, S., \& Ussher, S. (2005). Ticket to a sustainable future: an evaluation of the long term durability of the walking school bus program in Chrsitchurch, New Zealand. Transportation Policy, 12 (4), 314-323.

Kingham, S., \& Ussher, S. An assessment of the benefits of the walking school bus in Chrsitchurch, New Zealand. Transportation Research Part A: Policy and Practice. 2007, $41(6), 502-510$.

Larouche, R., \& Trudeau, F. (2010). Étude des impacts du transport actif sur la pratique d'activités physiques et la santé et de ses principaux déterminants. Science \& Sports, 25, 227-237. 
Larouche, R. (2012). The environmental and population health benefits of active transport: A review. In G. Liu (Ed.) Greenhouse Gases - Emissions, Measurement and Management (pp. 313-340). Rijeka, Croatia: InTech.

Larouche, R., Laurencelle, L., Shephard, R.J., \& Trudeau, F. (2012). Life transitions in the waning of physical activity from childhood to adult life: the Trois-Rivières study. Journal of Physical Activity and Health, 9, 516-524.

Larouche, R., \& Saunders T. (2012). Can active school transport prevent overweight and obesity in children and youth? Health Science Inquiry, 3, 64-65.

Lee, C., Zhu, X., Yoon, J., \& Varni, J.W. (2013). Beyond distance: children's school travel mode choice. Annals of Behavioral Medicine, 45 (Suppl. 1), S55-S67.

Lee, I.-M.., Shiroma, E.J., Lobelo P., Puska, P., Blair S.N., et al. (2012). Effect of physical inactivity on major non-communicable disease and life expectancy. Lancet, 380, 219229.

Lee, M.C., Orenstein, M.R., Richardson, M.C. (2008). Systematic review of active commuting to school and children's physical activity and weight. Journal of Physical Activity and Health. 5 (6), 930-949.

Lemieux, M., \& Godin, G. (2009). How well do cognitive and environmental variables predict active commuting? International Journal of Behavioral Nutrition and Physical Activity, 6,12 .

Levine, J.A, Eberhardt, N.L., Jensen, M.D. (1999). Role of nonexercise activity thermogenesis in resistance to fat gains in humans. Science, 283 (5399), 212-214.

Lewis, P. pour le Groupe de recherche Ville et mobilité (2008). Le transport actif et le système scolaire à Montréal et à Trois-Rivières : Analyse du système d'acteurs concernés par le 
transport actif des élèves des écoles primaires au Québec. Montréal, QC: Université de Montréal.

Litman, T. (2013). Transportation and public health. Annual Reviews of Public Health, 34, 217233.

Lobstein, T., Baur, L., \& Uauy, R. (2004). Obesity in children and young people: a crisis in public health. Obesity Reviews, 5 (Suppl. 1), 4-85.

Lorenc, T., Brunton, G., Oliver, S., Oliver, K., \& Oakley, A. (2008). Attitudes to walking and cycling among children, young people and parents: a systematic review. Journal of Epidemiology and Community Health, 62, 852-857.

Lubans, D.R., Boreham, C.A., Kelly, P., \& Foster, C.E. (2011). The relationship between active travel to school and health-related fitness in children and adolescents: a systematic review. International Journal of Behavioral Nutrition and Physical Activity, 8, 5.

Mackett, R., Brown, B., Gong, Y., Kitazawa, K., \& Paskins, J. (2007). Children's independent mobility in the local environment. Built Environment, 33 (4), 454-468.

Madsen, K.A., Gosliner, W., Woodward-Lopez, G., \& Crawford, P.B. (2009). Physical activity opportunities associated with fitness and weight status in adolescents in low-income communities. Archives of Pediatric and Adolescent Medicine. 163 (11), 1014-1021.

Maibach, E., Steg, L., \& Anable J. (2009). Promoting physical activity and reducing climate change: opportunities to replace short car trips with active transportation. Preventive Medicine, 49, 326-327.

Mammen, G., Faulkner, G., Buliung, R., \& Lay, J. (2012). Understanding the drive to escort: a cross-sectional analysis examining parental attitudes toward children's school travel and independent mobility. BMC Public Health, 12, 862. 
Marshall, J.D., Wilson, R.D., Meyer, K.L., Rajangam, S.K., McDonald, N.C., \& Wilson, E.J. (2010). Vehicle emissions during children's school commuting: Impact of education policy. Environmental Science \& Technology, 44 (5), 1537-1543.

Martin, S., \& Carlson, S. (2005). Barriers to children walking to or from school - United States, 2004. Morbidity and Mortality Weekly Reports, 54, 949-952.

Martin, S.L., Lee, S.M., \& Lowry, R. (2007). National prevalence and correlates of walking and bicycling to school. American Journal of Preventive Medicine, 33 (2), 98-105.

McCrindle, B.W., Manlhiot, C., Millar, K., Gibson, D., Stearne, K., et al. (2010). Population trends toward increasing cardiovascular risk factors in Canadian adolescents. Journal of Pediatrics, 157 (5), 837-843.

McDonald, N.C. (2007a). Travel and the social environment: evidence from Alameda County, California. Transportation Research Part D, 12, 53-63.

McDonald, N.C. (2007b). Active transportation to school: trends among U.S. schoolchildren, 1969-2001. American Journal of Preventive Medicine, 32, 509-516.

McDonald, N.C. (2008). Children's mode choice for the school trip: the role of distance and school location in walking to school. Transportation, 35, 23-35.

McDonald, N.C., \& Aalborg, A.E. (2009). Why parents drive children to school: Implications for Safe Routes to School programs. Journal of the American Planning Association, 75 (3), $331-342$.

McDonald, N.C., Brown, A.L., Marchetti, L.M., \& Pedroso, M.S. (2011). U.S. school travel, 2009: an assessment of trends. American Journal of Preventive Medicine, 41 (2), 146151. 
McDonald, N.C., Dwelley, A.E., Combs, T.S., Evenson, K.R., \& Winters, R.H. (2011).

Reliability and validity of the Safe Routes to School parent and students survey. International Journal of Behavioral Nutrition and Physical Activity, 8 (56).

McDonald, N.C. (2012). Is there a gender gap in school travel? An examination of US children and adolescents. Journal of Transport Geography, 20, 80-86.

McMillan, T. (2005). Urban form and a child's trip to school: The current literature and a framework for future research. Journal of Planning Literature, 19 (4), 440-456.

McMillan, T., Day, K., Boarnet, M., Alfonzo, M., \& Anderson, C. (2006). Johnny walks to school - does Jane? Sex differences in children's active travel to school. Children, Youth and Environments, 16 (1), 75-89.

McMillan, T. (2007). The relative influence of urban form on a child's travel mode to school. Transportation Research Part A, 41, 69-79.

McMinn, D., Rowe, D., Murtagh, S., \& Nelson, N.M. (2012). The effect of a school-based active commuting intervention on children's commuting physical activity and daily physical activity. Preventive Medicine, 54 (5), 316-318.

Mendoza, J.A., Watson, K., Baranowski, T., Nicklas, T.A., Uscanga, D.K., \& Hanfling, M.J. (2011). The walking school bus and children's physical activity: a pilot cluster randomized controlled trial. Pediatrics, 138 (3), e537-e544.

Mitra, R., Buliung, R., \& Faulkner, G. (2010). Spatial clustering and the temporal mobility of walking school trips in the Greater Toronto Area. Health \& Place, 16, 646-650.

Mitra, R., \& Buliung, R.N. (2012). Built environment correlates of active school transportation: neighbourhood and the modifiable areal unit problem. Journal of Transport Geography, 20, 51-61. 
Mitra, R., \& Faulkner, G. (2012). There's no such thing as bad weather, just the wrong clothing: climate, weather and active school transportation in Toronto, Canada. Canadian Journal of Public Health, 103 (Suppl. 3), S35-S41.

Morency, C., \& Demers, M. (2010). Active transportation as a way to increase physical activity among children. Child: Care, Health and Development, 36 (3), 421-427.

Mota, J., Gomes, H., Almeida, M., Ribeiro, J.C., Carvalho, J., \& Santos, M.P. (2007). Active versus passive transportation to school - differences in screen time, socio-economic position and perceived environmental characteristics in adolescent girls. Annals of Human Biology, 34 (3), 273-282.

Murtagh, S., Rowe, D.A., Elliott, M.A., McMinn, D., \& Neslon, N.M. (2012). Predicting active school travel: the role of planned behavior and habit strength. International Journal of Behavioural Nutrition and Physical Activity, 9, 65.

Napier, M.A., Brown, B.B., Werner, C.M., \& Gallimore, J. (2011). Walking to school: community design and child and parent barriers. Journal of Environmental Psychology, $31(1), 45-51$.

Nelson, N.M., Foley, E., O’Gorman, D.J., Moyna, N.M., \& Woods, C.B. (2008). Active commuting to school: how far is too far? International Journal of Behavioural Nutrition and Physical Activity, 5, 1.

Norman, G.J., Nutter, S.K., Ryan, S., Sallis, J.F., Calfas, K.J., \& Patrick, K. (2006). Community design and access to recreational facilities as correlates of adolescent physical activity and body-mass index. Journal of Physical Activity and Health, 3 (Suppl.1), S118-S128.

Olds, T.S. (2009). One million skinfolds: secular trends in the fatness of young people 19512004. European Journal of Clinical Nutrition, 63, 934-946. 
O’Loghlen, S., Pickett, W., \& Janssen, I. (2011). Active transportation environments surrounding Canadian schools. Canadian Journal of Public Health, 102 (5), 364-368.

Orsini, A.F., \& O'Brien, C. (2006). Fun, fast and fit: influences and motivators for teenagers who cycle to school. Children, Youth and Environments, 16 (1), 121-132.

Østergaard, L., Grøntved, A., Borrestad, L.A.B., Froberg, K., Gravesen, M., \& Andersen, L.B. (2012). Cycling to school is associated with lower BMI and lower odds of being overweight or obese in a large population-based study of Danish adolescents. Journal of Physical Activity and Health, 9 (5), 617-625.

Pabayo, R., \& Gauvin, L. (2008). Proportion of students who use various modes of transportation to and from school in a representative population-based sample of children and adolescents, 1999. Preventive Medicine, 46 (1), 63-66.

Pabayo, R., Gauvin, L., \& Barnett, T.A. (2011). Longitudinal changes in active transportation to school in Canadian children aged 6 through 16 years. Pediatrics, 128 (2), e404-413.

Pabayo, R., Gauvin, L., Barnett, T.A., Morency, P., Nikiéma, B., \& Séguin, L. (2012a). Understanding the determinants of active transportation to school among children: evidence of environmental injustice from the Quebec longitudinal study of child development. Health \& Place, 18 (2), 163-171.

Pabayo, R., Maximova, K., Spence, J.C., Vander Ploeg, K., Wu, B., \& Veugelers, P.J. (2012b). The importance of active transportation to and from school for daily physical activity among children. Preventive Medicine, 55 (3), 196-200.

Pacilli, M.G., Giovannelli, I., Prezza, M., \& Augimeri, M.L. (in press). Children and the public realm: antecedents and consequences of independent mobility in a group of $11-13$-yearold Italian children. Children's Geographies. 
Page, A., Cooper, A.R., Griew, P., \& Jago, R. (2010). Independent mobility, perceptions of the built environment, and children's participation in play, active travel and structured exercise and sport: the PEACH project. International Journal of Behavioral Nutrition and Physical Activity, 7, 1.

Panter, J.R., Jones, A.P., \& van Sluijs, E.M.F. (2008). Environmental determinants of active travel in youth: a review and framework for future research. International Journal of Behavioral Nutrition and Physical Activity, 5, 34.

Panter, J.R., Jones, A.P., van Sluijs, E.M.F., \& Griffin, S.J. (2010a). Attitudes, social support and environmental perceptions as predictors of active commuting behaviour in school children. Journal of Epidemiology and Community Health, 64, 41-48.

Panter, J.R., Jones, A.P., van Sluijs, E.M.F., \& Griffin, S.J. (2010b). Neighborhood, route and school environments and children's active commuting. American Journal of Preventive Medicine, 38 (3), 268-278.

Panter, J.R., Jones, A.P., van Sluijs, E.M.F., \& Griffin, S.J. (2011). The influence of distance to school on the associations between active commuting and physical activity. Pediatric Exercise Science, 23 (1), 72-86.

Pizarro, A.N., Ribeiro, J.C., Marques, E.A., Mota, J., \& Santos, M.P. (2013). Is walking to school associated with improved metabolic health? International Journal of Behavioral Nutrition and Physical Activity, 10, 12.

Pont, K., Ziviani, J., Wadley, D., \& Abbott, R. (2011). The model of children's active travel (MCAT): A conceptual framework for examining factors influencing children's active travel. Australian Occupational Therapy Journal, 58, 138-144. 
Prezza, M., Pilloni, S., Morabito, C., Sersante, C., Alparone, F., \& Giuliani, M. (2001). The influence of psychosocial and environmental factors on children's independent mobility and relationship to peer frequentation. Journal of Community \& Applied Social Psychology, 11, 435-450.

Pucher, J., \& Buehler, R. (2008). Making cycling irresistible: Lessons from the Netherlands, Denmark and Germany. Transport Reviews, 28 (4), 495-528.

Rainham, D.G., Bates, C.J., Blanchard, C.M., Dummer, T.J., Kirk, S.F., \& Shearer, C.L. (2012) Spatial classification of youth physical activity patterns. American Journal of Preventive Medicine, 42 (5), e87-e96.

Ridley, K., Ainsworth, B.E., Olds, T.S. (2008). Development of a Compendium of Energy Expenditures for Youth. International Journal of Behavioral Nutrition and Physical Activity, 5, 45.

Rissotto, A., \& Tonucci, F. (2002). Freedom of movement and environmental knowledge in elementary school children. Journal of Environmental Psychology, 22, 65-77.

Roberts, I. (1995). Walking to school has future benefits. British Medical Journal, 312, 1229.

Roberts, I., Carlin, J., Bennett, C., Bergstrom, E., Guyer, B. et al. (1997). An international study of the exposure of children to traffic. Injury Prevention, 3, 89-93.

Rossen, L.M., Pollack, K.M., Curriero, F.C., Shields, T.M., Smart, M.J., Furr-Holden, D.M., \& Cooley-Strickland, M. (2011). Neighborhood incivilities, perceived neighborhood safety, and walking to school among urban-dwelling children. Journal of Physical Activity and Health, 8 (2), 262-271.

Saelens, B.E., \& Handy, S. (2008). Built environment correlates of walking: a review. Medicine \& Science in Sports \& Exercise, 40 (Suppl. 7), S550-S566. 
Saksvig, B.I., Webber, L.S., Elder, J.P., Ward, D., Evenson, K.R., et al. (2012). A cross-sectional and longitudinal study of travel by walking before and after school among eigth-grade girls. Journal of Adolescent Health, 51 (6), 608-614.

Sallis, J.F. (2000). Age-related decline in physical activity: a synthesis of human and animal studies. Medicine \& Science in Sports \& Exercise, 32 (9), 1598-1600.

Sallis, J.F., Cervero, R.B., Ascher, W., Hendersen, K.A., Kraft, M.J., \& Kerr, J. (2006). An ecological approach to creating active living communities. Annual Reviews of Public Health, 27, 297-322.

Sallis, J.F., Bowles, H.R., Bauman, A., Ainsworth, B.E., Bull, F.C., \& Craig, C.L. (2009). Neighborhood environments and physical activity among adults in 11 countries. American Journal of Preventive Medicine, 36 (6), 484-90.

Sallis, J.F., Floyd, M.F., Rodriguez, D.A., \& Saelens, B.E. (2012). Role of built environments in physical activity, obesity, and cardiovascular disease. Circulation, 125, 729-737.

Seliske, L., Pickett, W., \& Janssen, I. (2012). Urban sprawl and its relationship with active transportation, physical activity and obesity in Canadian youth. Health Reports, 23 (2), $17-25$.

Shephard, R.J. (2008). Is active commuting the answer to population health? Sports Medicine, 38 (9), 751-758.

Sirard, J.R., Riner, W.F. Jr., McKiver, K.L., \& Pate, R.R. (2005). Physical activity and active commuting to elementary school. Medicine \& Science in Sports \& Exercise, 37 (12), 2062-2069.

Sirard, J.R., Alhassan, S., Spencer, T.R., \& Robinson, T.N. (2008). Changes in physical activity from walking to school. Journal of Nutrition and Education Behavior, 40 (5), 324-326. 
Smith, L., Sahlqvist, S., Ogilvie, D., Jones, A., Griffin, S.J., \& van Sluijs, E. (2012). Is active travel to non-school destinations associated with physical activity in primary school children? Preventive Medicine, 54, 224-228.

Smith, L., Sahlqvist, S., Ogilvie, D., Jones, A., Corder, K., Griffin, S.J., \& van Sluijs, E. (2012). Is a change in mode of travel to school associated with a change in overall physical activity levels in children? Longitudinal results from the SPEEDY study. International Journal of Behavioral Nutrition and Physical Activity, 9, 134.

Southward, E.F., Page, A.S., Wheeler, B.W., \& Cooper, A.R. (2012). Contribution of the school journey to daily physical activity in children aged 11-12 years. American Journal of Preventive Medicine, 43 (2), 201-204.

Stamatakis, E., Wardle, J., \& Cole, T.J. (2010). Childhood obesity and overweight trends in England: evidence for growing socioeconomic disparities. International Journal of Obesity. 34, 41-47.

Stewart, O. (2011). Findings from research on active transportation to school and implications for Safe Routes to School programs. Journal of Planning Literature, 26 (2), 127-150.

Stewart, O., Moudon, A.V., Claybrooke, C. (2012). Common ground: eight factors that influence walking and biking to school. Transport Policy, 21, 240-248.

Stone, M.R., Faulkner G.E., \& Buliung, R.N. (2013). How active are children in Toronto? A comparison with accelerometry data from the Canadian Health Measures Survey. Chronic Disease in Canada, 33 (2), 61-68.

Timperio, A., Crawford, D., Telford, A., \& Salmon, J. (2004). Perceptions about the local neighborhood and walking and cycling among children. Preventive Medicine, 38, 39-47. 
Timperio, A., Ball, K., Salmon, J., Roberts, R., Giles-Corti, B. et al. (2006). Personal, family, social, and environmental correlates of active commuting to school. American Journal of Preventive Medicine, 30 (1), 45-51.

Tomkinson, G.R., Léger, L.A., Olds, T.S., \& Cazorla, G. (2003). Secular trends in the performance of children and adolescents (1980-2000): An analysis of 55 studies of the $20 \mathrm{~m}$ shuttle run test in 11 countries. Sports Medicine, 33 (4), 285-300.

Trang, N.N.H.D., Hong, T.K., Dibley, M.J., \& Sibbritt, D.W. (2009). Factors associated with physical inactivity in adolescents in Ho Chi Minh City, Vietnam. Medicine \& Science in Sports and Exercise, 41 (7), 1374-1383.

Trang, N.H.H.D., Hong, T.K., \& Dibley, M.J. (2012). Active commuting to school among adolescents in Ho Chi Minh City, Vietnam: changes and predictors in a longitudinal study, 2004 to 2009. American Journal of Preventive Medicine, 42 (2), 120-128.

Trapp, G.S.A., Giles-Corti, B., Christian, H.E., Bulsara, M., Timperio, A.F., McCormack, G.R., \& Villaneuva, K.P. (2011). On your bike! A cross-sectional study of the individual, social and environmental correlates of cycling to school. International Journal of Behavioral Nutrition and Physical Activity, 8, 123.

Tremblay, M.S., Esliger, D.W., Tremblay, A., \& Colley, R. (2007). Incidental movement, lifestyle-embedded physical activity and sleep: new frontiers in physical activity assessment. Canadian Journal of Public Health, 98 (Suppl. 2), S208-S216.

Tremblay, M.S., Shields, M., Laviolette, M., Craig, C.L., Janssen, I., \& Connor Gorber, S. (2010). Fitness of Canadian children and youth: Results from the 2007-2009 Canadian Health Measures Survey. Health Reports, 21 (1), 1-14. 
Tremblay, M.S., LeBlanc, A.G., Kho, M., Saunders, T.J., Larouche, R., Colley, R.C., et al. (2011). Systematic review of sedentary behaviour and health indicators in school-aged children and youth. International Journal of Behavioral Nutrition and Physical Activity, $8,98$.

Tremblay, M.S., Warburton, D.E.R., Janssen, I., Paterson, D.H., Latimer, A.E., et al. (2011). New Canadian physical activity guidelines. Applied Physiology Nutrition and Metabolism, 36 (1), 36-46.

Troiano, R., Berrigan, D., Dodd, K.W., Mâsse, L.C., Tilert, T., \& McDowell, M. (2008). Physical activity in the United States measured by accelerometer. Medicine and Science in Sports \& Exercise, 40, 181-188.

Trudeau, F., \& Shephard, R.J. (2008). Physical education, school physical activity, school sports and academic performance. International Journal of Behavioral Nutrition and Physical Activity, 5, 10.

Tudor-Locke, C., Ainsworth, B.E., \& Popkin, B.M. (2001). Active commuting to school: An overlooked source of children's physical activity? Sports Medicine, 31 (5), 309-313.

Tudor-Locke, C., Neff, L.G., Ainsworth, B.E., Addy, C.L., \& Popkin, B.M. (2002). Omission of active commuting to school and the prevalence of children's health-related physical activity level: the Russian Longitudinal Monitoring Study. Child: Care, Health, and Development, 28 (6), 507-512.

Turner, L., Chriqui, J., \& Chaloupka, F. (In press). Walking school bus programs in U.S. public elementary schools. Journal of Physical Activity and Health. 
Unger, N., Bond T.C., Wang, J.S., Koch, D.M., Menon, S. et al. (2010). Attribution of climate forcing to economic sectors. Proceedings of the National Academy of Science, 107 (8), $3382-3387$.

United States Department of Health and Human Services. (2008). Physical Activity Guidelines Advisory Committee Report, 2008. Washington, DC: United States Department of Health and Human Services.

Valentine, G. (1997). "Oh yes I can." "Oh no you can't": Children and parent's understanding of kids' competence to negotiate public space safely. Antipode, 29, 65-89.

van der Ploeg, H.P., Merom, D., Corpuz, G., \& Bauman, A.E. (2008). Trends in Australian children travelling to school 1971-2003: Burning petrol or carbohydrates? Preventive Medicine, 46, 60-62.

Van Mechelen, W., Twisk, J.W.R., Bertheke Post, G., Snel, J., \& Kemper, H.C.G. (2000). Physical activity of young people: the Amsterdam Longitudinal Growth and Health Study. Medicine \& Science in Sports \& Exercise, 32 (9), 1610-1616.

van Sluijs, E.M.F., Fearne, V.A., Mattocks, C., Riddoch, C., Griffin, S.J., \& Ness, A. (2009). The contribution of active travel to children's physical activity levels: Cross-sectional results from the ALSPAC study. Preventive Medicine, 48, 519-524.

Voorhees, C.C., Ashwood, S., Evenson, K.R., Sirard, J.R., Rung, A.L., et al. (2010). Neighborhood design and perceptions: Relationship with active commuting. Medicine and Science in Sports \& Exercise, 41 (7), 1253-1260.

Watson, M., \& Dannenberg, A.L. (2008). Investments in Safe Routes to School projects: Public health benefits for the larger community. Preventing Chronic Disease, 5 (3), 1-7. 
Wedderkopp, N., Froberg, K., Hansen, H.S., \& Anderson, L.B. (2004). Secular trends in physical fitness and obesity in Danish 9-years-old girls and boys: Odense School Child Study and Danish substudy of the European Youth Heart Study. Scandinavian Journal of Medicine and Science in Sports, 14, 150-155.

Westerstahl, M., Barnekow-Bergkvist, M., Hedberg, G., \& Jansson I. (2003). Secular trends in body dimensions and physical fitness among adolescents in Sweden from 1974 to 1995. Scandinavian Journal of Medicine and Science in Sports, 13 (2), 128-137.

Westerterp, K.R. (2010). Physical activity, food intake, and body weight regulation: insights from doubly labeled water studies. Nutrition Reviews. 68 (3), 148-154.

Wong, B.Y.M., Faulkner, G., Buliung, R., \& Irving, H. (2011a). Mode shifting in school travel mode: examining the prevalence and correlates of active school transport in Ontario, Canada. BMC Public Health, 11, 618.

Wong, B.Y.M., Faulkner, G., \& Buliung, R. (2011b). GIS measured environmental correlates of active school transport: A systematic review of 14 studies. International Journal of Behavioral Nutrition and Physical Activity, 8, 39.

Woodcock, J., Edwards, P., Tonne, C., Armstrong, B.G., Ashiru, O., et al. (2009). Public health benefits of strategies to reduce greenhouse-gas emissions: urban land transport. Lancet, 374 (9705), 1930-1943.

World Health Organization. (2013). Global status report on road safety 2013: supporting a decade of action. Geneva, Switzerland: World Health Organization.

Yancey, A.K., \& Sallis, J.F. (2009). Physical activity: Cinderella or Rodney Dangerfield? Preventive Medicine, 49 (9), 277-279. 


\section{Appendix 1. Contributions}

Study 1. All authors contributed to the search strategy with input from a professional librarian

(Dr. Margaret Sampson). Richard Larouche executed the search strategy, examined the resulting papers for inclusion/exclusion criteria, extracted relevant information from the included papers and assessed the quality of evidence of included papers. Travis Saunders examined the resulting papers for inclusion/exclusion criteria, and performed duplicate data extraction and quality assessment for a subsample of papers. Richard Larouche wrote the first draft of the manuscript and all authors reviewed the manuscript and provided feedback. A consensus was reach between all authors for the attribution of GRADE ratings.

Study 2. Richard Larouche was involved in data collection, conducted all data analyses and wrote the first draft of the manuscript. Meghann Lloyd and Mark Tremblay designed the study, obtained funding, and completed the REB screening process. Emily Knight was the research coordinator for the study, and Meghann Lloyd was involved in data collection. All authors reviewed the manuscript and provided feedback.

Study 3. Richard Larouche obtained approval from Statistics Canada to perform secondary data analyses at the University of Ottawa Research Data Center and wrote the first draft of the manuscript. Mark Tremblay has been employed by Statistics Canada for the design of the CHMS. All authors were involved in the design of the analyses, reviewed the manuscript and provided feedback. 
Study 4, 5 and 6. Richard Larouche coordinated data collection, performed all analyses and wrote the first draft of the manuscripts. Richard Larouche and Mark Tremblay obtained ethics approval from the University of Ottawa and Children's Hospital of Eastern Ontario REBs and from the Ottawa-Carleton Research Advisory Committee (Appendix 3-5). All authors were involved in the design of the study, reviewed the manuscripts and provided feedback. 


\section{Appendix 2. List of abbreviations}

AST: Active school transport

BMI: Body mass index

CAPL: Canadian Assessment of Physical Literacy

CHMS: Canadian Health Measures Survey

CVD: Cardiovascular disease

MVPA: Moderate-to-vigorous physical activity

PA: Physical activity

REB: Research Ethics Board

SES: Socioeconomic status

UK: United Kingdom

US: United States 


\section{Appendix 3. University of Ottawa Research Ethics Board (REB) approval}

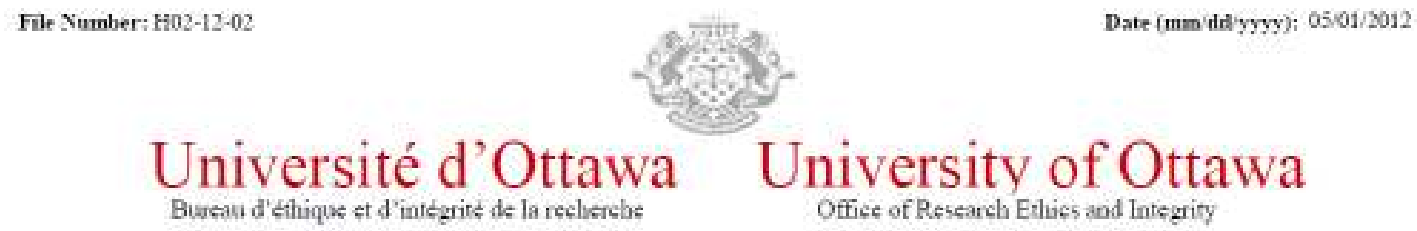

\section{Ethics Approval Notice}

\section{Health Sciences and Science REB}

\section{Principal Investigator / Supervisor / Co-investigator(s) / Student(s)}

$\begin{array}{llll}\text { First Name } & \text { Last Name } & \text { Amliation } & \text { Role } \\ \text { Mark } & \text { Trembliny } & \text { Health Sciences/Others } & \text { Supervisor } \\ \text { Richard } & \text { Laronche } & \text { Health Sciences/Human Kinetics } & \text { Student Researcher }\end{array}$

File Number: $\mathrm{H} 02-12-02$

Type of Project: PhD Thesis

Title: Active Transportation Transition Study

$\begin{array}{lll}\text { Approval Date (mm/dd/yyy) } & \text { Expiry Date (mm/dd/yyyy) } & \text { Approval Type } \\ 03 / 01 / 2012 & 02 / 28 / 2013 & \text { Ja }\end{array}$

(Ia: Approval, Ib: Approval for initial stage only, I: Couditional approval)

Special Conditions / Comments:

Approval (as of May 1", 2012) $\rightarrow$ Permissions from CHEO, the Ottawa Catholic School Board, and the Ottawa-Carleton District School Board have been received.

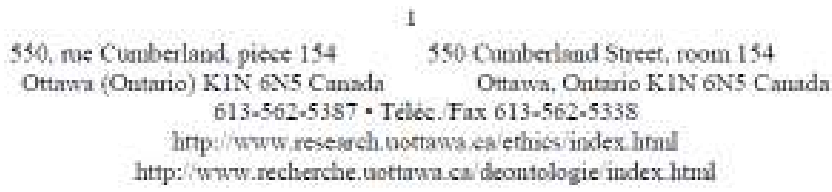


This is to confirut flat the University of Ortawa Research Elhics Board identified above, which operates in accordance with the Tri-Council Policy Statement and other applicalble laws and regulations in Ontario, has examined and approved the applicatiou for ethical approval for the above naned research project as of the Ethics Approval Date indicared for the period above and subject to the couditions listed the section above eacitled "Special Conditions/ Conunents".

During the course of the study the protocol may not be modified withou prior written approval from the REB excepe when necessary to remove subjects from inneediate endmagennent or when the modification(s) penain to only administrative or logistical components of the srady (eg change of telephoue number). Investigators must also proumptly alent the REB of any chnages which increase the risk to participant(s), any changes which considerably affect che condnct of the project. all manticipated and hannfil evears that occur. and new iaformation that may negarively affect the conduct of the project and safety of the participant(s). Modifications to the project, information/consent docnmentation, and or recruitment docmenentation. shonld be submitted to this office for ayproval ising the "Modification to research project" fonis available at:

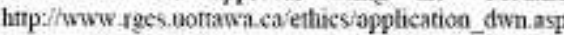

Please sabmit an aumal status report to the Protocol Officer four weeks before the above-referenced expiry date to either close the file or request a renewal of ethics approval. This document can be found at: lutp:/www res wottawa ca ethics/application_dwn.asp

If you have any questious, please do not besitate to conacr the Ethics Office at extension 5387 or by e-mail at:

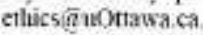

Signature:

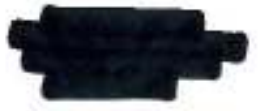

Kum Thompson

Protocol Officer for Ethics in Researcl

For Daniel Lagarec, Claair of the Health Sciences and Sciences RFB

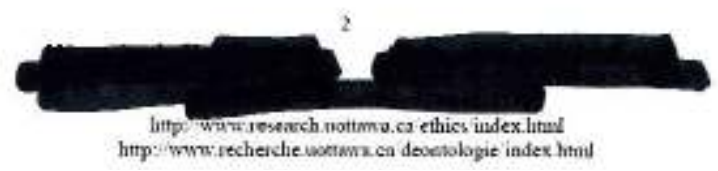




\section{Appendix 4. Children's Hospital of Eastern Ontario REB approval}

\section{Appendix 4. Children's Hospital of Eastern Ontario REB approval}
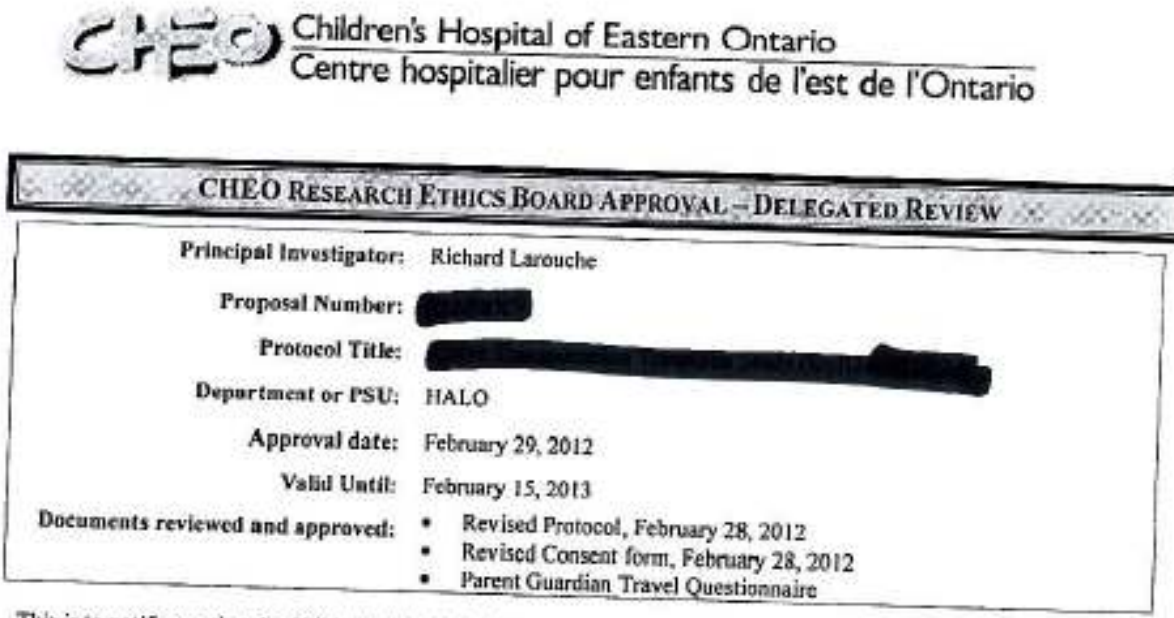

This is to notify you that the Children's Hospital of Esstem Ontario Research Ethics Board has granted appeoval to the which is reserved for ptudy on the date noted above, Your project was reviewed under the delegated review saream. which is reserved for projects that involve no more than minimal risk to haman subjects. Final approval is granted for the above noted study, with the understanding that the investigntor agrees to comply what
the following requirements: - The investigator must conduct the study in compliance with the protocol and any additional conditions set out by the
Baard.

- The investigator must nat implement any deviation fram, or ehanges to, the protocol without the approval of the REB, or when the change involves anly logistical or administrative sspects of the study (c.g., change of telephene
number or resetirch stafm. number or resetrirch stafm - The investigator must, prior to use, subsair to the Baard changes to the study documentation, e.g., changes to the
informed consent letters, recruitment materials.

For all other resessrth studies, investigators must promptly report to the KEB B all tnexpected and untoward occurrences (including the loss of theft of study data and other such privacy breaches).

- Investigators mast mubmit an anoual ronewal report to the REB 30 days prior to the expira).

- Investigators must submit a final report at the coort the REB 30 days prior to the expiration date stated above.

- Investigators must provite the Boport as the canclusion of the study.

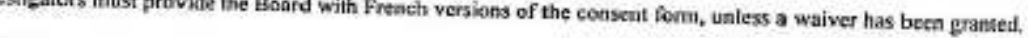

For complete procedunes relatiog to these modifications. please refer to the REB website at

7600 ext 2128 .

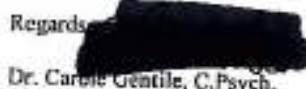

Chair, Research Ethies Board

CG/smeh $2902 / 2012$

c.c. CHEO RI Administration

Dr. Mark Tremblay, Drector, HALO

University of Ottawa Ethics Department

This is an official docamemt. Please retain the orikinal for your file 2010 versiour
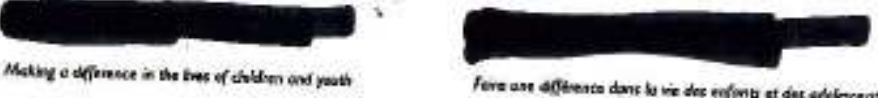


\section{Appendix 5. Ottawa-Carleton Research Advisory Committee approval}

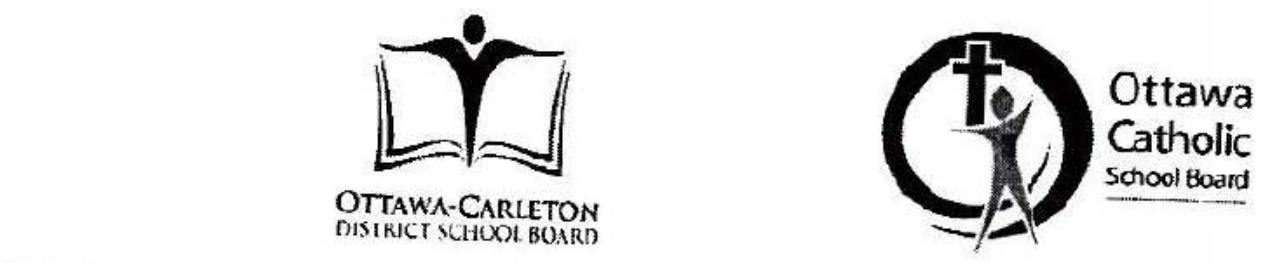

\section{Ottawa-Carleton Research Advisory Committee}

April 30, 2012

Richard Larouche

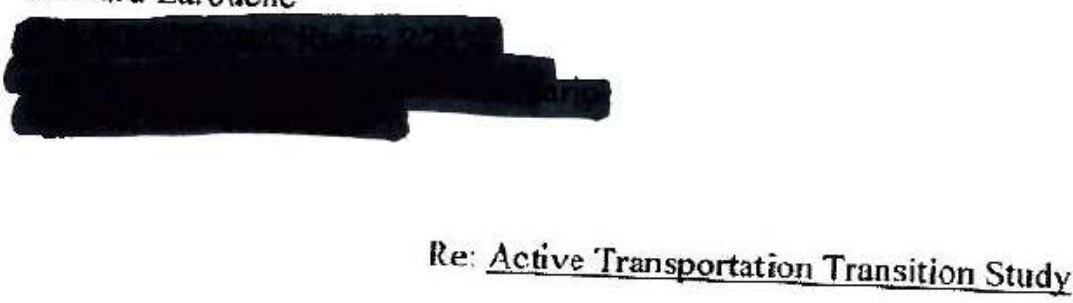

Dear Mr. Larouche,

The Ottawa-Carleton Rescarch Advisory Committee (OCRAC) informed you of the outcome of your research application in a letter dated March 26, 2012. According to the letter of response, Ottawa-Carleton District School Board and the and clarifications for implementation in both the

In your e-mail correspondence sent to me on April 18,2012, you have made the required inform you that your research proposal informed consent and parent questionnaire. I am pleased to in both school boards.

Thank you for showing research interest in our schools.

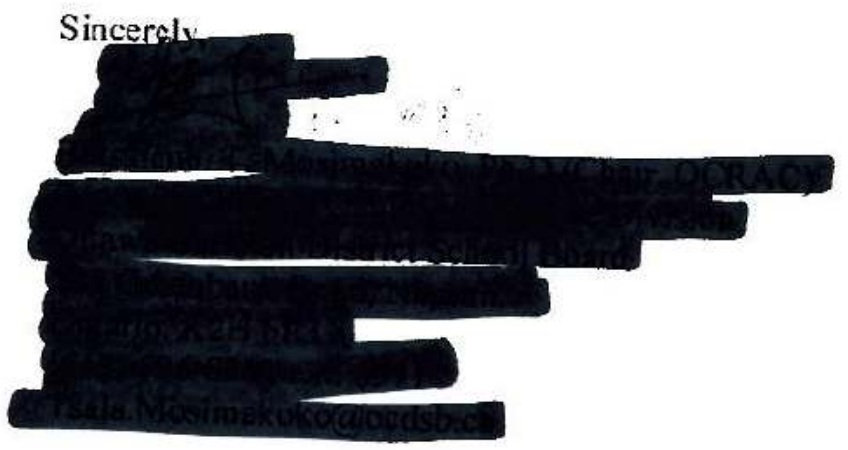




\section{Appendix 6. Transportation diary}

ID:

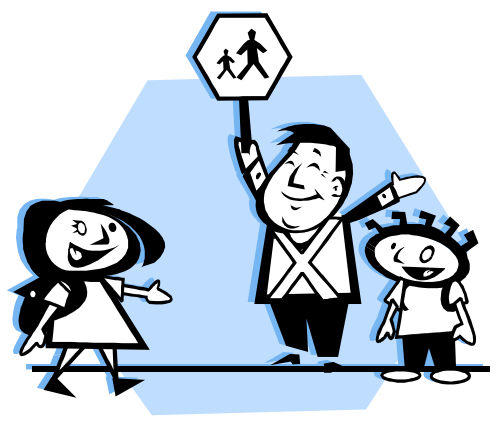

Date:
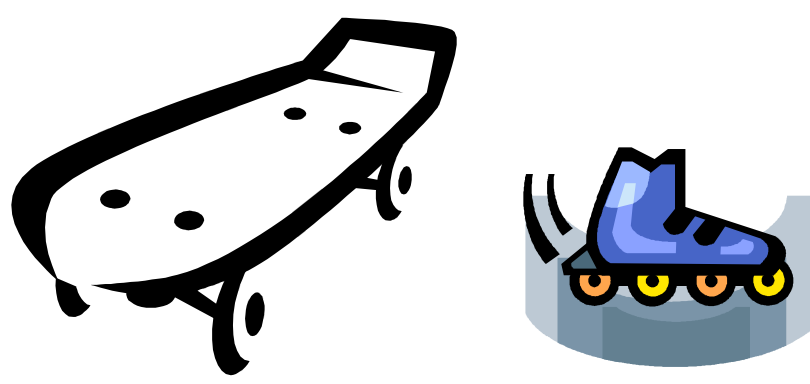

Can you draw an $\underline{\mathbf{X}}$ in the table below to indicate how you travelled to school on each day of the week? (If you missed school, please don't put an X)

\begin{tabular}{|l|l|l|l|l|l|l|l|}
\hline & Walk & Bike & Skateboard & Roller-blade & Car & Bus & Other \\
\hline Monday & & & & & & & \\
\hline Tuesday & & & & & & & \\
\hline Wednesday & & & & & & & \\
\hline Thursday & & & & & & & \\
\hline Friday & & & & & & & \\
\hline
\end{tabular}
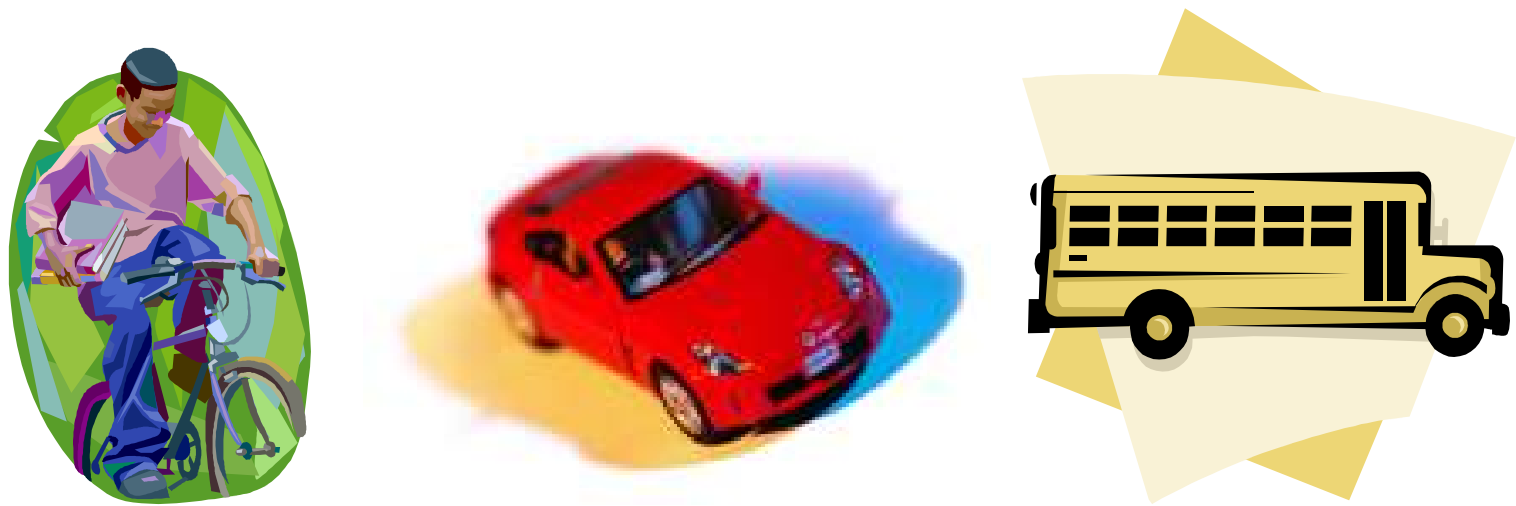

Can you indicate by an $\underline{\mathbf{X}}$ in the table below how you travelled back home on each day of the week? (If you missed school, please don't put an X)

\begin{tabular}{|l|l|l|l|l|l|l|l|}
\hline & Walk & Bike & Skateboard & Roller-blade & Car & Bus & Other \\
\hline Monday & & & & & & & \\
\hline Tuesday & & & & & & & \\
\hline Wednesday & & & & & & & \\
\hline Thursday & & & & & & & \\
\hline Friday & & & & & & & \\
\hline
\end{tabular}




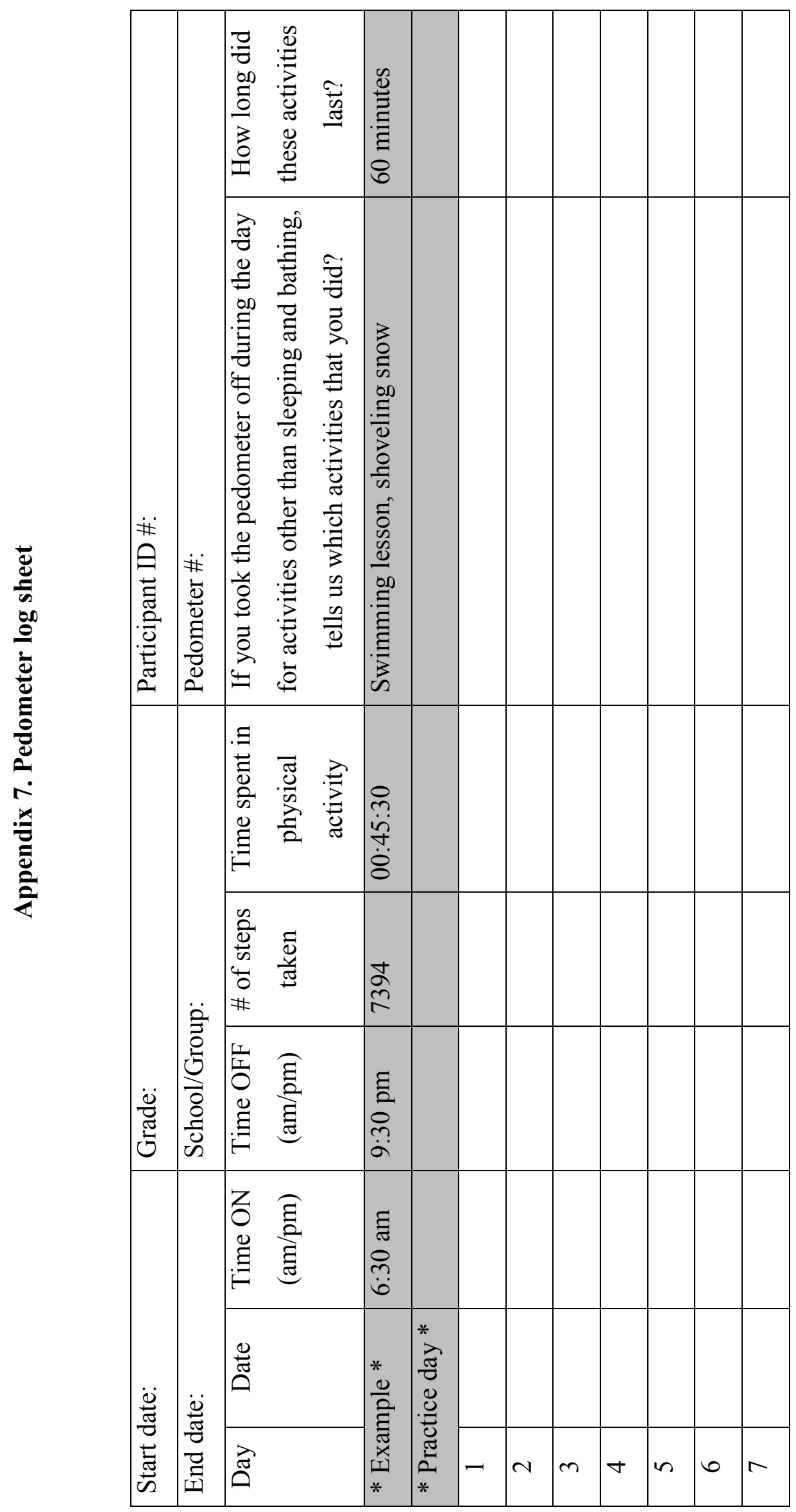




\section{Appendix 8. Parent/guardian travel questionnaire}

How does your child usually gets to and from school? Please write down the number of days per week. For every line, the total must add up to 5.

\begin{tabular}{|c|c|c|c|c|c|c|c|}
\hline Season & $\begin{array}{c}\text { Travel } \\
\text { direction }\end{array}$ & Walk & Cycle & By car & $\begin{array}{c}\text { By bus / } \\
\text { public transit }\end{array}$ & Other & Total \\
\hline $\begin{array}{c}\text { Spring } \\
\text { (April- } \\
\text { June) }\end{array}$ & To school & & & & & & $=5$ days \\
\cline { 2 - 7 } & $\begin{array}{c}\text { From } \\
\text { school }\end{array}$ & & & & & & $=5$ days \\
\hline
\end{tabular}

** If you have written "other", please specify which mode of transport it is:

\section{PART B: TELL US ABOUT YOUR HOUSEHOLD}

1) Do you speak a language other than English at home?
$\square$ yes
$\square$ no

2) What is this language?

(For example: French, Spanish, Arabic, Mandarin, etc.)

3) How many automobiles in working order (e.g., cars, trucks, vans, SUV's) are there in your household?
$\square$ none
$\square$ one
two
three
$\square$ four or more

4) Does your child own or have access to a bicycle?
$\square$ Yes
$\square$ No

5) In total, how many children live in your household?

Please list the number of children in the following age groups:

$0-3$ years:

4-5 years:

6-9 years:

10-11 years:

12-13 years:

$14-15$ years:

$>16$ years: 
6) My family is a:

$\checkmark$ two-parent family

single-parent family

$\checkmark$ other, please describe your family:

7) What is the highest level of education that the child's father has completed?

elementary school (grades 1-8)

secondary school (grades 9-12)

college

university

graduate school

not applicable

8) What is the highest level of education that the child's mother has completed?

elementary school (grades 1-8)

secondary school (grades 9-12)

college

university

graduate school

not applicable

9) Currently, the child's father is?

employed full-time

employed part-time

student full-time

student part-time

at home with children

without paid employment

not applicable

10) Currently, the child's mother is?

employed full-time

employed part-time

student full-time

student part-time

at home with children

without paid employment

not applicable

Can you indicate your postal code on the line below? [We will only use this information to estimate the distance between your residence and your child's school]

Can you indicate your home phone number and the name of the school your children will go to next year to ensure that we can contact you in September?

Phone number:

School: 
PART C: TELL US IF YOU THINK THAT YOUR CHILD FACES THE FOLLOWING BARRIERS TO WALKING OR BIKING TO SCHOOL

Please indicate your level of agreement with the statements written in the table below. Please CHECK ( $\downarrow$ ) ONE number only.

\begin{tabular}{|c|c|c|c|c|}
\hline 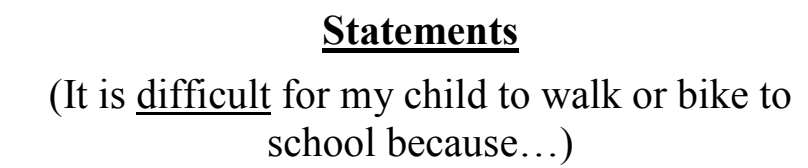 & $\begin{array}{l}1 \\
\text { Strongly } \\
\text { disagree }\end{array}$ & $\begin{array}{l}2 \\
\text { Somewhat } \\
\text { disagree }\end{array}$ & $\begin{array}{l}3 \\
\text { Somewhat } \\
\text { agree }\end{array}$ & $\begin{array}{l}4 \\
\text { Strongly } \\
\text { agree }\end{array}$ \\
\hline \multicolumn{5}{|l|}{ There are too many hills along the way } \\
\hline \multicolumn{5}{|l|}{ There are no sidewalks or bike lanes } \\
\hline \multicolumn{5}{|l|}{ The route is boring } \\
\hline \multicolumn{5}{|l|}{ The route does not have good lighting } \\
\hline \multicolumn{5}{|l|}{ There is too much traffic along the route } \\
\hline \multicolumn{5}{|l|}{ There is too much traffic around our home } \\
\hline \multicolumn{5}{|l|}{ There is too much traffic around the school } \\
\hline \multicolumn{5}{|l|}{ There is one or more dangerous crossing } \\
\hline \multicolumn{5}{|l|}{ My child gets too hot and sweaty } \\
\hline \multicolumn{5}{|l|}{ No other children walk or bike to school } \\
\hline \multicolumn{5}{|l|}{ It's not considered cool to walk or bike } \\
\hline \multicolumn{5}{|l|}{ My child has too much stuff to carry } \\
\hline \multicolumn{5}{|l|}{$\begin{array}{l}\text { It is easier for me to drive my child here on the } \\
\text { way to something else }\end{array}$} \\
\hline \multicolumn{5}{|l|}{ It involves too much planning ahead } \\
\hline \multicolumn{5}{|l|}{$\begin{array}{l}\text { It is unsafe because of crime (strangers, gangs, } \\
\text { drugs) }\end{array}$} \\
\hline \multicolumn{5}{|l|}{ My child gets bullied, teased, harassed } \\
\hline \multicolumn{5}{|l|}{ There is nowhere to leave a bike safely } \\
\hline \multicolumn{5}{|l|}{ There are stray dogs } \\
\hline It is too far & & & & \\
\hline
\end{tabular}

Note: added elements are written in italic. 


\section{PART E: TELL US ABOUT HOW YOU CAME TO LIVE IN YOUR NEIGHBOURHOOD}

The following statements describe some common reasons why a household might choose to live in a neighbourhood. Please tell us which ones applied to your household's neighborhood choice. Please CHECK ( $\sqrt{ }$ ) ONE number only. 1 = you agree completely; 5 = you completely disagree;

$3=$ you are not sure

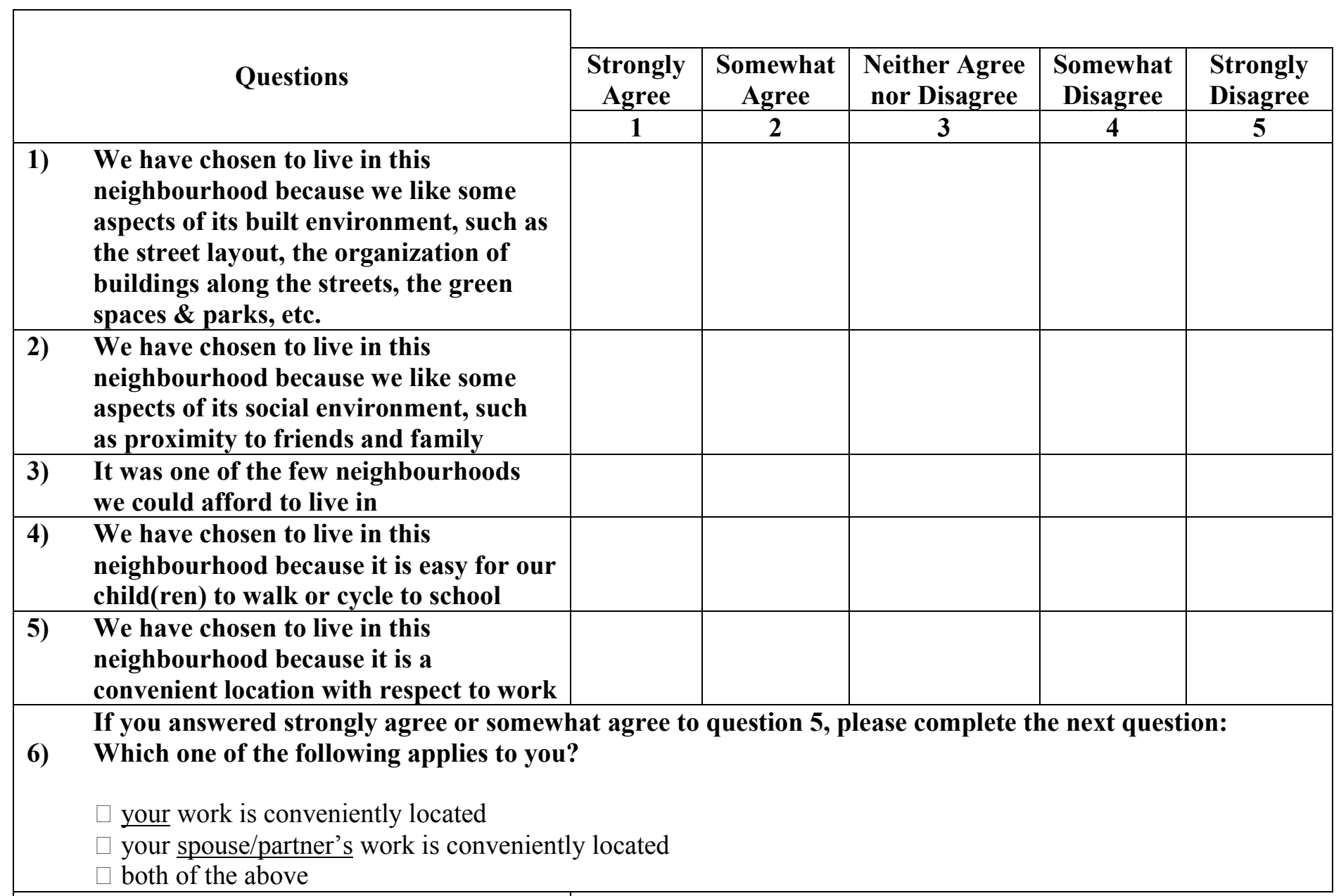

7) We have chosen to live in this neighbourhood because it is a convenient location with respect to our household's non-work activities, such as shopping, exercise, parks, etc.

8) We have chosen to live in this neighbourhood because there are shops and restaurants you can walk or cycle to

\begin{tabular}{|c|c|c|c|c|}
\hline $\begin{array}{c}\text { Strongly } \\
\text { Agree }\end{array}$ & $\begin{array}{c}\text { Somewhat } \\
\text { Agree }\end{array}$ & $\begin{array}{c}\text { Neither Agree } \\
\text { nor Disagree }\end{array}$ & $\begin{array}{c}\text { Somewhat } \\
\text { Disagree }\end{array}$ & $\begin{array}{c}\text { Strongly } \\
\text { Disagree }\end{array}$ \\
\hline 1 & 2 & 3 & 4 & 5 \\
& & & & \\
& & & & \\
& & & & \\
& & & & \\
& & & & \\
\end{tabular}




\section{Appendix 9. Child questionnaire on perceived barriers to active school transport}

Please indicate your level of agreement with the statements written in the first column of the table below.

Please CHECK $(\sqrt{ })$ ONE number only. ( 1 = you strongly disagree; 4 = you strongly agree)

\begin{tabular}{|c|c|c|c|c|}
\hline $\begin{array}{c}\underline{\text { Statements }} \\
\text { (It is } \underline{\text { difficult for me to walk or bike to school }} \\
\text { because...) }\end{array}$ & $\begin{array}{l}1 \\
\text { Strongly } \\
\text { disagree }\end{array}$ & $\begin{array}{l}2 \\
\text { Somewhat } \\
\text { disagree }\end{array}$ & $\begin{array}{c}3 \\
\text { Somewhat } \\
\text { agree }\end{array}$ & $\begin{array}{c}4 \\
\text { Strongly } \\
\text { agree }\end{array}$ \\
\hline \multicolumn{5}{|l|}{ There are too many hills along the way } \\
\hline \multicolumn{5}{|l|}{ There are no sidewalks or bike lanes } \\
\hline \multicolumn{5}{|l|}{ The route is boring } \\
\hline \multicolumn{5}{|l|}{ The route does not have good lighting } \\
\hline \multicolumn{5}{|l|}{ There is too much traffic along the route } \\
\hline \multicolumn{5}{|l|}{ There is too much traffic around our home } \\
\hline \multicolumn{5}{|l|}{ There is too much traffic around the school } \\
\hline \multicolumn{5}{|l|}{ There is one or more dangerous crossing } \\
\hline \multicolumn{5}{|l|}{ I get too hot and sweaty } \\
\hline \multicolumn{5}{|l|}{ No other children walk or bike to school } \\
\hline \multicolumn{5}{|l|}{ It's not considered cool to walk or bike } \\
\hline \multicolumn{5}{|l|}{ I have too much stuff to carry } \\
\hline \multicolumn{5}{|l|}{$\begin{array}{l}\text { It is easier for my parents to drive me on the way } \\
\text { to something else }\end{array}$} \\
\hline \multicolumn{5}{|l|}{ It involves too much planning ahead } \\
\hline \multicolumn{5}{|l|}{$\begin{array}{l}\text { It is unsafe because of crime (strangers, gangs, } \\
\text { drugs) }\end{array}$} \\
\hline \multicolumn{5}{|l|}{ I get bullied, teased, harassed } \\
\hline \multicolumn{5}{|l|}{ There is nowhere to leave a bike safely } \\
\hline \multicolumn{5}{|l|}{ There are stray dogs } \\
\hline It is too far & & & & \\
\hline
\end{tabular}




\section{Appendix 10. Internal consistency of perceived barriers to active school transport}

\begin{tabular}{|c|c|c|c|c|c|}
\hline Period & Individual & Subscale & Subsample (n) & Mean (SD) & Cronbach $\boldsymbol{\alpha}$ \\
\hline \multirow{4}{*}{ Baseline } & \multirow{3}{*}{ Child } & Environment & 42 & $1.75(.58)$ & .67 \\
\cline { 3 - 6 } & & Planning & 42 & $1.44(.45)$ & .69 \\
\cline { 3 - 6 } & \multirow{3}{*}{ Parent } & Safety & 43 & $1.29(.52)$ & .71 \\
\cline { 3 - 6 } & & Environment & 40 & $1.63(.69)$ & .84 \\
\cline { 3 - 6 } & & Planning & 38 & $1.35(.47)$ & .73 \\
\cline { 3 - 6 } & \multirow{3}{*}{ Child } & Safety & 40 & $1.12(.29)$ & .67 \\
\hline \multirow{4}{*}{ Follow-up } & Environment & 28 & $1.74(.68)$ & .83 \\
\cline { 3 - 6 } & \multirow{3}{*}{ Parent } & Planning & 29 & $1.49(.52)$ & .77 \\
\cline { 3 - 6 } & & Safety & 29 & $1.38(.65)$ & .89 \\
\cline { 3 - 6 } & & Environment & 28 & $1.77(.73)$ & .80 \\
\cline { 3 - 6 } & & Planning & 27 & $1.45(.74)$ & .88 \\
\cline { 3 - 6 } & & Safety & 27 & $1.20(.34)$ & .60 \\
\hline
\end{tabular}

Note: Children and parents completed Forman's (2008) 17-item questionnaire on perceived barriers to AST from which three subscales are derived. The Likert-type statements range from 1 (strongly disagree) to 4 (strongly agree). Cronbach's alpha $(\alpha)$ was computed among the subsample of participants who responded to all items included in the subscales. 